Vierter Beratungsgegenstand:

\title{
Die Anforderungen an ein zukunftsfähiges Infrastrukturrecht
}

\author{
1. Referat von Professor Dr. Oliver Dörr, LL.M., Osnabrück*
}

Inhalt

Seite

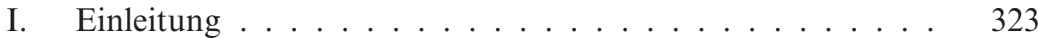

II. Begriff und Konzept . . . . . . . . . . . . . . . . . . . . . . . . . . . . . . . . . 325

1. Zur Begriffsgeschichte . . . . . . . . . . . . . . . 326

2. „Infrastruktur" als Rechtsbegriff . . . . . . . . . . . . 328

3. Infrastruktur und Daseinsvorsorge . . . . . . . . . . . . 332

4. Europäische Konzepte . . . . . . . . . . . . . . . . 335

III. Die Infrastrukturverantwortung des Staates . . . . . . . . . . 337

1. Verfassungsrechtliche Grundlagen. . . . . . . . . . . . 337

2. Infrastruktur als Gegenstand von Gewährleistungsverantwortung . . . . . . . . . . . . 340

3. Verfassungsrechtliche Ausgestaltungsdeterminanten . . . . 347

4. Fragen des Verantwortungsinhalts. . . . . . . . . . . . . . . . 349

IV. Der überstaatliche Rahmen . . . . . . . . . . . . . . . . . . . 354

1. Infrastrukturverantwortung und Unionsrecht . . . . . . 354

a) EU-Infrastrukturpolitik. . . . . . . . . . . . . . . . . 354

b) EU-Binnenmarktrecht . . . . . . . . . . . . . . 355

c) Daseinsvorsorge als Verfassungswert der Union . . . . . 358

2. Infrastruktur im Völkerrecht . . . . . . . . . . . . . . . . 359

V. Schluss ...................... 361

\section{Einleitung}

Liegt es nicht auf der Hand, was die A1, der Elbdeich bei Magdeburg, die Breitbandnetze der Telekom, Windkraftanlagen im Emsland, ein Klärwerk in Sachsen, das Ratsgymnasium in Osnabrück, der städtische

* Für hilfreiche Anmerkungen und Kritik danke ich Pascale Cancik, Thomas Groß, Jens-Peter Schneider und Indra Spiecker gen. Döhmann. 
Friedhof in Köln-Dünnwald und der Hauptbahnhof in Berlin gemeinsam haben? Und was sie mit der Stadtmauer Jerichos, den Aquädukten Roms und den mittelalterlichen Stromregalien ${ }^{1}$ verbindet?

Sie alle sind mit einem „Megawort" der modernen Industriegesellschaft in Bezug genommen, über das der Historiker Dirk van Laak notierte: „Infrastruktur gehört zu jenen ,Plastikwörtern“ der wissenschaftlich-technischen Zivilisation, mit denen wir heute soziale Sicherheit, staatliche Verantwortung und wirtschaftliche Prosperität konnotieren“.2 „Infrastruktur" ist ständig und überall, es ist ein allgegenwärtiger Begriff der Alltags-, Politik-, Verwaltungs- und Rechtssprache, es ist Gegenstand von Forschung und Lehre $^{3}$ - und wird dabei regelmäßig mehr assoziativ als mit einem klaren Begriffsinhalt verwendet. Ohne präzise Definition durchzieht der Begriff das deutsche Gesetzesrecht ebenso wie das europäische Unionsrecht, gerne auch adjektivisch als ,infrastrukturell“4 oder ,infrastrukturbasiert“ ( 2 Abs. 3 Nr. 3 TKG), als Plural: die „Infrastrukturen“5, mit adjektivischen Zusätzen, wie z.B. „ländliche“ oder „frühkindliche Infrastruktur“ (§ 3 Abs. 1 ZuInvG) oder „kritische Infrastrukturen“ ( $\$ 2$ Abs. 2 Nr. 3 Satz 4 ROG).

Die Allgegenwart von Infrastruktur, wie auch ihr existentieller Charakter ermöglichen aus rechtswissenschaftlicher Perspektive eine Vielzahl von

\footnotetext{
${ }^{1} \mathrm{Zu}$ diesen z.B. S. Reinheimer Das Verbindungskonzept der Bundeswasserstraßenverwaltung, 2008, 35-39; Chr. Gröpl in: Maunz/Dürig, GG, Art. 89 Rn. 3 (2007).

2 D. van Laak Infra-Strukturgeschichte, Geschichte und Gesellschaft 27 (2001), 367.

${ }^{3}$ Etwa in Gestalt von Lehrstühlen und Instituten für Infrastruktur und Infrastrukturrecht, wie z.B. dem Lehrstuhl für Öffentliches Recht, Europäisches Informationsund Infrastrukturrecht an der Albert-Ludwigs-Universität in Freiburg i.Br. (Schneider), dem Lehrstuhl für Öffentliches Recht, Immobilienrecht, Infrastrukturrecht und Informationsrecht an der Universität Regensburg (Kühling), der Professur Verwaltungsrecht, insbes. Regulierungs- und Infrastrukturrecht an der Universität Potsdam (ausgeschrieben im September 2013), dem Lehrstuhl für Verkehrs- und Infrastrukturplanung an der TU Dresden (Ahrens), dem Ostseeinstitut für Seerecht, Umweltrecht und Infrastrukturrecht der Universität Rostock, dem Institut für Infrastruktur und Ressourcenmanagement an der Universität Leipzig, dem Institut für Infrastruktur an der Universität Innsbruck oder dem Fachgebiet Wirtschafts- und Infrastrukturpolitik an der TU Berlin (Fakultät Wirtschaft und Management). Als Gegenstand des juristischen Studiums z.B. im Schwerpunktbereich „Öffentliches Wirtschafts- und Infrastrukturrecht" an der LMU München; bis 2012 im Schwerpunkt „Wirtschaftsverwaltungsrecht und Infrastrukturrecht" an der Leibniz Universität Hannover.

4 Vgl. z.B. in § 2 Abs. 2 Nr. 1 ROG (,infrastrukturelle Verhältnisse“), § 136 Abs. 3 Nr. 2c BauGB (,infrastrukturelle Erschließung“), Anlage 1 Nr. 13.12 UVPG (,infrastrukturelle Hafenanlage“). Im Schrifttum z.B. P. Lerche Infrastrukturelle Verfassungsaufträge, FS Friauf, 1996, 251.

5 Wie z.B. in $\S 2$ Abs. 2 Nr. 3 ROG, $\S 1$ Abs. 1 AEG, § 28a EnWG, §§ 1, 15a Abs. 2 und in den Überschriften zu $§ \S 77 \mathrm{a}, 77 \mathrm{~b}$ TKG.
} 
Zugriffen auf das mir gestellte Thema. Da kommt es sehr gelegen, dass Teilaspekte bereits Gegenstand vergangener Tagungen waren: vor allem auf der Hallenser Tagung 1994 zur Privatisierung von Verwaltungsaufgaben $^{6}$, in St. Gallen 2002 zur Öffentlichen Gemeinwohlverantwortung ${ }^{7}$, aber auch in Berlin 2010 (Verbraucherschutz durch Regulierungsrecht) ${ }^{8}$ und letztes Jahr in Kiel zu den Herausforderungen durch Großvorhaben ${ }^{9}$.

Ich muss mich hier - auch in Absprache mit Herrn Wißmann - im wesentlichen auf zwei Aspekte beschränken: die besondere Rolle des Staates in Bezug auf die Infrastrukturversorgung (III.) und die überstaatliche Einbindung dieser Rolle (IV.). Zuvor aber soll das Proprium der Rechtswissenschaft, die Suche nach präzisen Begriffen und Begriffsinhalten, zu seinem Recht kommen (II.). Aus der sachlichen Erörterung müssen sich dabei die Aspekte der „Zukunftsfähigkeit“ ergeben, da dieser eine eigenständige rechtliche Bedeutung wohl nur schwer beizulegen ist. ${ }^{10}$

\section{Begriff und Konzept}

$\mathrm{Zu}$ den Anforderungen an ein ,Zukunftsfähiges Infrastrukturrecht“ gehört, dass Gesetzgebung und Rechtswissenschaft ein möglichst präzises Verständnis von seinem Gegenstand entwickeln. Bislang ist weder dieser Gegenstand hinreichend klar abgegrenzt, noch das Infrastrukturrecht selbst als ein eigenständiges, geschweige denn kohärentes Rechtsgebiet konturiert.

Über die Wissenschaftsdisziplinen hinweg besteht seit vielen Jahrzehnten Einigkeit, dass es eine allgemein akzeptierte Definition des Begriffes „Infrastruktur“ nicht gibt. ${ }^{11}$ Der Schweizer Ökonom René L. Frey schrieb

\footnotetext{
${ }^{6}$ J. Hengstschläger, L. Osterloh, H. Bauer und T. Jaag Privatisierung von Verwaltungsaufgaben, VVDStRL 54 (1995), 166, 204, 243 bzw. 287.

7 M. Heintzen und A. Voßkuhle Beteiligung Privater an der Wahrnehmung öffentlicher Aufgaben und staatliche Verantwortung, VVDStRL 62 (2003), 220 bzw. 266.

8 J. Hellermann und W. Durner Schutz der Verbraucher durch Regulierungsrecht, VVDStRL 70 (2011), 366 bzw. 398.

${ }^{9}$ K. Waechter und Th. Mann Großvorhaben als Herausforderung für den demokratischen Rechtsstaat, VVDStRL 72 (2013), 499 bzw. 544.

${ }^{10}$ Vgl. G. Beaucamp Das Konzept der zukunftsfähigen Entwicklung im Recht, 2002.

11 Vgl. z.B. R. L. Frey Infrastruktur, in: Handwörterbuch der Wirtschaftswissenschaft, Bd. 4, 1978, 200 (201); U. E. Simonis Zur inhaltlich-systematischen Deutung des Begriffes „Infrastruktur“, Zeitschrift für Ganzheitsforschung 27 (1983), 119 (120); $K .-W$. Schatz Zur Entwicklung des Begriffs Infrastruktur, in: H. Berger (Hrsg.) Wettbewerb und Infrastruktur in Post- und Telekommunikationsmärkten, ZögU 1996, Beiheft 19, 122 (125); G. Hermes Staatliche Infrastrukturverantwortung, 1998, 168; R. Stober
} 
bereits 1970, dass unter „Infrastruktur“ alles Denkbare, nur selten etwas Präzises verstanden werde, ${ }^{12}$ und die mehr als vierzig Jahre, die seit diesem Diktum vergangen sind, haben an der Richtigkeit dieser Beobachtung kaum etwas geändert.

\section{Zur Begriffsgeschichte}

Wie van Laak herausgearbeitet hat, ist der Begriff „Infrastruktur“ als neulateinischer Neologismus um 1875 im französischen Sprachraum entstanden. ${ }^{13}$ „Infrastructure“ bezeichnete anlässlich der Überprüfung eines Eisenbahnprojekts den bodenverhafteten Unterbau von Eisenbahn-Konstruktionen, dem die „superstructure“, also die darüber errichteten Vorrichtungen (Signale, Oberleitungen, Bahnhöfe etc.) gegenübergestellt wurden. Noch in der ersten Hälfte des 20. Jahrhunderts wurde der Begriff vor allem für ortsfeste Anlagen im Dienste der Mobilität verwendet, während er ab den frühen 1950er Jahren in die Planungssprache der NATO einging und hier eine erste Ausweitung auf militärische Anlagen erfuhr. 1954 erschien der Begriff im deutschen „Brockhaus“ und erlangte damit als deutsches Lehnwort lexikalischen Rang. ${ }^{14}$ Auf internationaler Ebene fand er in den 1950er Jahren außerdem Eingang in die Sprache der frühen Entwicklungshilfepolitik und hier in einem deutlich weiteren Sinne Verwendung: Umfasst waren etwa auch staatliche Maßnahmen der Sozial-, Bildungs-, Gesundheitspolitik und der Stadtplanung.

$\mathrm{Zu}$ Beginn der 1960er Jahre entdeckte die deutschsprachige Wissenschaft den Begriff „Infrastruktur“, zunächst wohl vor allem die Sozialund Wirtschaftswissenschaften, die den Begriff in ihre disziplinäre Terminologie aufnahmen. ${ }^{15}$ Beschrieben wurde damit eine der Basisfunktionen

in: Wolff/Bachof/Stober/Kluth, Verwaltungsrecht I, 12. Aufl. 2007, §4 Rn. 17; A. Glöckner Kommunale Infrastrukturverantwortung und Konzessionsmodelle, 2009, 6. Vgl. auch G. Hünnekens Rechtsfragen der wirtschaftlichen Infrastruktur, 1995, 7: „ein offener und nahezu beliebig dehnbarer Begriff".

12 R. L. Frey Infrastruktur. Grundlagen der Planung öffentlicher Investitionen, 1970, im Vorwort.

${ }^{13}$ Zum folgenden D. van Laak Der Begriff „Infrastruktur“ und was er vor seiner Erfindung besagte, Archiv für Begriffsgeschichte 41 (1999), 280 (280-290).

${ }_{14} \operatorname{Van} \operatorname{Laak}$ (Fn. 13), 282 zitiert hierfür aus Der große Brockhaus, Bd. 5 (1954).

15 R. Jochimsen definierte Infrastruktur sehr weit als die Gesamtheit aller materiellen, institutionellen und personellen Anlagen, Einrichtungen und Gegebenheiten, die der arbeitsteiligen Wirtschaft zur Verfügung stehen und dazu beitragen, die Entgelte für gleiche Leistungen der Produktivkräfte auszugleichen und zugleich die größte Zuwachsrate der Gesamtwirtschaft herbeizuführen, vgl. Theorie der Infrastruktur, 1966, 100 und 145 . 
einer Volkswirtschaft, die den Integrationsgrad und das Niveau der Wirtschaftstätigkeit bestimmen: Infrastrukturen besitzen hier eine Vorleistungsfunktion, ihr Nutzen geht über die bloße Inanspruchnahme hinaus. In der Soziologie erlaubte die Entlehnung des französischen Begriffs „,infrastructure“ Theoriebildungen zum „Unter- und Oberbau“ einer Gesellschaft, Infrastruktur erhielt hier damit einen nicht anlagen- oder gegenstandsbezogenen Begriffsinhalt. ${ }^{16}$

In verschiedenen Wissenschaftsdisziplinen, auch im Recht, wird der Begriff Infrastruktur praktisch immer zur Bezeichnung bekannter Anwendungsbeispiele verwendet, nicht aber als Ergebnis einer wirklichen (erschöpfenden) Definition oder einer normativen Ableitung. ${ }^{17}$ Der Begriffsinhalt ist also vor allem assoziativ oder empirisch angereichert, nicht normativ konturiert. Klassisch bezieht sich Infrastruktur auf die sächlichen Grundlagen von Verkehr und Kommunikation, Energie- und Wasserversorgung sowie Entsorgung - man mag hierin den interdisziplinären Begriffskern erkennen. Üblicherweise ausgeklammert werden Sicherheitseinrichtungen, die Justiz und die öffentliche Verwaltung. ${ }^{18}$ Darüber hinaus finden sich weitergehende Begriffskontexte, wie z.B. ,personelle Infrastruktur" ${ }^{19}$, „soziale Infrastruktur“20, „technische Infrastruktur“"21, „Informationsinfrastruktur“"22, ,Telematikinfrastruktur“"23, „organisatorische Infrastruktur“ (§ 128 Abs. 5 S. 2 SGB XII); „,kulturelle Infrastruktur“24, „geistige Infrastruktur" ${ }^{\text {"25, Gesundheitsinfrastruktur }}{ }^{26}$, Bildungs-

16 Vgl. van Laak (Fn. 13), 288.

17 So z.B. auch in BVerfGE 38, 258 (270 f.) mit dem Zusatz ,Infrastruktur im weiteren Sinne“. In BVerfGE 97, 332 (343) wird ein städtischer Kindergarten als „Infrastruktureinrichtung" bezeichnet. Auch Bildungs- und Pflegeeinrichtungen fasst das BVerfG unter den Begriff, vgl. die Nachweise bei Glöckner Infrastrukturverantwortung (Fn. 11), 12.

18 Vgl. Frey (Fn. 12), 2; Frey (Fn. 11), 202.

19 Vgl. z.B. Jochimsen (Fn. 15), 133; Simonis (Fn. 11), 122; Schatz (Fn. 11), 134; Glöckner Infrastrukturverantwortung (Fn. 11), 6.

20 Vgl. z.B. K. Stern Staatsrecht I, 1984, § 21 II.2; Glöckner Infrastrukturverantwortung (Fn. 11), 7 und 13; M. Schuler-Harms und A. Hense in: Fehling/Ruffert (Hrsg.) Regulierungsrecht, 2009, $\S 15$ und $\S 16$. Rechtsverbindlich in $\S 2$ Abs. 2 Nr. 3 S. 2 ROG.

$21 \S 291$ a Abs. 5a S. 5 SGB V, § 128 Abs. 5 S. 2 SGB XII.

22 F. Schoch Öffentlich-rechtliche Rahmenbedingungen einer Informationsordnung, VVDStRL 57 (1998), 158 (198).

$23 \S 87$ Abs. 1 S. 6, 291a, 291b SGB V.

24 Vgl. z.B. Frey (Fn. 11), 202; Schatz (Fn. 11), 134 unten; van Laak (Fn. 2), 376 unten. Mit Rechtsverbindlichkeit in Art. 35 Abs. 7 des deutsch-deutschen Einigungsvertrages 1990 .

25 Chr. Gramm Privatisierung und notwendige Staatsaufgaben, 2001, 334.

26 Stober in: Wolff/Bachof/Stober/Kluth, Verwaltungsrecht I (Fn. 11), § 4 Rn. 23. 
infrastruktur ${ }^{27}$, „normative Infrastruktur“ ${ }^{28}$ oder „Vertrauensinfrastruktur"29. Sogar die Umwelt kommt als „natürliche oder gewachsene Infrastruktur" vor ${ }^{30}$.

\section{2. „Infrastruktur“ als Rechtsbegriff}

Angesichts dieser terminologischen Weite und empirisch radizierten Beliebigkeit mag man fragen, ob „Infrastruktur“ überhaupt zum Rechtsbegriff taugt. Sein assoziativer Charakter ist nur dort von vornherein unschädlich, wo der Begriff lediglich in Überschriften zur schlagwortartigen Kennzeichnung des Gemeinten verwendet wird ${ }^{31}$, nicht aber als ein Gesetzesbegriff, unter den die Subsumtion eröffnet ist. Nichtsdestotrotz ist „Infrastruktur“ heute ein geläufiger Terminus der Gesetzgebung, und zwar sowohl im europäischen Unionsrecht als auch in deutschen Gesetzen. Zuerst verwendet wurde er in Deutschland wohl in $\S 1$ Abs. 1 Nr. 2 des GRW-Gesetzes von $1969^{32}$. Dann fand er sich wieder im Zusammenhang mit der Zeitenwende von 1989/90, z.B. in § 4 PostStruktG 1989 und im Einigungsvertrag von $1990^{33}$. Heute taucht er im Gesetz vor allem im Zusammenhang mit regulierten Wirtschaftssektoren auf, über welche die Einwirkung des Unionsrechts hinweggebraust ist (AEG, EnWG, LuftVG, TKG), außerdem im Planungsrecht (§ 2 Abs. 2 Nr. 2-4, $\S 8$ Abs. 5 Nr. 3 ROG) sowie vereinzelt im Sozial- und Wettbewerbsrecht ${ }^{34}$. Das Grundgesetz kennt den Begriff „Infrastruktur“ nach wie vor nicht.

$27 \S 3$ Abs. 1 Nr. 1 des Gesetzes zur Umsetzung von Zukunftsinvestitionen der Kommunen und Länder (ZuInvG) v. 2.3.2009 (BGBl. I 416, 428); R. Herzog in: Maunzl Dürig, GG, Art. 20, VIII Rn. 13 (1980).

${ }_{28}$ Z.B. U. Tranow Das Konzept der Solidarität. Handlungstheoretische Fundierung eines soziologischen Schlüsselbegriffs, 2012, 111; M. Kotzur Daseinsvorsorge als Unionsaufgabe, JöR n.F. 61 (2013), 195 (210).

29 C. Franzius Gewährleistung im Recht, 2009, 650.

${ }^{30}$ Bei Frey (Fn. 11), 202.

${ }^{31}$ Wie z.B. im Titel des „Gesetzes zur Beschleunigung von Planungsverfahren für Infrastrukturvorhaben“v. 9.12.2006 (BGBl. I 2833) und in den Überschriften zu $\$ \S 77 \mathrm{a}$, 77b TKG.

${ }^{32}$ Gesetz über die Gemeinschaftsaufgabe „Verbesserung der regionalen Wirtschaftsstruktur“ (GRW-Gesetz) v. 6.10.1969 (BGBl. I 1861). Darin ging es um die „Förderung des Ausbaus der Infrastruktur", soweit es für die Entwicklung der gewerblichen Wirtschaft erforderlich ist. In der heute geltenden Fassung der Norm ist die Rede von der „Förderung der wirtschaftsnahen Infrastruktur".

${ }_{33}$ Art. 28 Abs. 2, 2. Spiegelstrich nannte die „wirtschaftsnahe Infrastruktur“, Art. 35 Abs. 7 die „kulturelle Infrastruktur“.

${ }^{34}$ Vgl. z.B. §§ 291a, 291b SGB V („,technische Infrastruktur“, „Telematikinfrastruktur"); $§ 19$ Abs. 2 Nr. 4 und Anlage zu $\S 98$ Nr. 4, Nr. 4 GWB. 
Eine Begriffsklärung erleichtert die ökonomische Theorie: Diese charakterisiert Infrastruktur meist anhand verschiedener typischer Merkmale, und zwar solcher technischer, ökonomischer und institutioneller Art: Technisch geht es regelmäßig um immobile, unteilbare Anlagen mit einer langen Lebensdauer und eine standortgebundene Nutzung; ökonomisch sind wesentlich eine Kostendegression durch economies of scale, ausgeprägte externe Effekte, ein hoher Fix- und Gemeinkostenanteil, ein großer Investitionsumfang und hohes Investitionsrisiko; institutionell zeichnet sich die Infrastruktur dadurch aus, dass die Betriebsführung regelmäßig defizitär ist, keine Marktpreise zur Anwendung kommen, die Planung, Herstellung und Betriebsführung zentral organisiert sind. ${ }^{35}$

Begriffsprägend sind also zunächst vor allem zwei Elemente: die unmittelbare Allgemeinnützigkeit und der Versorgungszweck. Infrastruktur bezeichnet ein öffentliches Gut, das Gegenstand einer öffentlichen Investition bzw. dessen Betrieb öffentlich verantwortet ist. Es ist im Rahmen seiner Funktionsbestimmung grundsätzlich für jedermann zugänglich. Sein Funktionszweck ist gerichtet auf die Versorgung der Allgemeinheit und schafft sächliche Voraussetzungen für die Erfüllung ihrer Grundbedürfnisse. Es handelt sich m.a.W. um ein „kollektives Medium der Subsistenz". ${ }^{36}$ Hinzu kommt als drittes Merkmal die Ortsgebunden-

35 Vgl. grundlegend J. Stohler Zur rationalen Planung der Infrastruktur, Konjunkturpolitik 11 (1965), 279 (281); daran anschließend z.B Frey (Fn. 12), 1 und ders. (Fn. 11), 201; Simonis (Fn. 11), 120; Schatz (Fn. 11), 128 f.

36 Van Laak (Fn. 13), 290; ders. (Fn. 2), 371. Es ist das begriffsimmanente Element der öffentlichen Versorgung, das Infrastruktur und Infrastrukturrecht besonders anfällig macht für emotionalisierte öffentliche Debatten, deren Verlauf und Auswirkungen ihren sachlichen Anlass oft weit hinter sich lassen: Die öffentliche Aufregung über den Umbau des Stuttgarter Hauptbahnhofs, dem ein abgeschlossenes Planungsverfahren zugrunde lag, führte 2010/11 zu grundsätzlichen Debatten über die Gestaltung solcher Verfahren, ja über den Zustand des demokratischen Gemeinwesens sowie die soziologischen Besonderheiten von Teilen der Bevölkerung Baden-Württembergs (vgl. z.B. Th. Groß Stuttgart 21: Folgerungen für Demokratie und Verwaltungsverfahren, DÖV 2011, 510; W. Leisner Stuttgart 21. „Wir sind das Volk!“ - Wer?, NJW 2011, 33; F. Walter Alt, stur, egoistisch, Spiegel online v. 8.9.2011). Die augenscheinlich gesteuerte Aufregung über einen Richtlinienentwurf der Europäischen Kommission zur transparenten Vergabe öffentlicher Konzessionen mündete in eine europäische Bürgerinitiative zum Menschenrecht auf Wasser (,Right2Water") und brachte den Entwurf, der im Kern schlicht eine Angleichung der Konzessions- an die Auftragsvergabe bezweckt hatte, politisch zu Fall (dazu z.B. H. Kafsack Angst ums Wasserglas, FAZ v. 8.3.2013, 11; M. K. Wagner Verwässert, F.A.S. v. 28.4.2013, 8; H. Kafsack Die Geschichte eines Missverständnisses, FAZ v. 26.6.2013, 10). Die aktuellen Debatten über eine Rekommunalisierung der kommunalen Energieversorgung (z.B. in Hamburg und Berlin) scheinen neben der plebiszitären Steuerung - sehr stark vom Wunsch nach einer am Gemeinwohl orientierten Versorgung getrieben, übersehen dabei aber die Eigenrationalitäten des gel- 
heit, welche die Verlagerung oder den Import von Infrastruktur verhindert und ihre wirtschaftliche Nutzung und Wirkungsweise maßgeblich beeinflusst: Infrastruktur ist immer raumwirksam und betrifft immer die Nutzung von Boden und Grundeigentum.

Wir bezeichnen mit „Infrastruktur“ also ortsfeste Anlagen und Einrichtungen, welche der Versorgung der Bevölkerung mit lebensnotwendigen Gütern und Dienstleistungen dienen (z.B. mit Wasser, Energie, Mobilität, Kommunikation, Bildung, Gesundheits- und Sozialdiensten, wohl auch mit Bargeld und Finanzdienstleistungen), im Prinzip jedermann zugänglich sind und deren Betrieb von der öffentlichen Hand verantwortet wird.

Schon in der volkswirtschaftlichen Theorie ist allerdings nicht ganz klar, ob der Infrastrukturbegriff auf die „Hardware“ der öffentlichen Versorgung beschränkt ist oder ob die Leistungen (Dienste), die mit Hilfe dieser Anlagen erbracht werden, ebenfalls davon umfasst sind. Gelegentlich unterscheidet die Ökonomie hier das Infrastruktursachkapital, also die Anlagen und Einrichtungen, als Infrastruktur im engeren Sinne vom output dieser Subsektoren als Infrastruktur im weiteren Sinne. ${ }^{37}$ Auch in der Polit- und Alltagssprache sowie im rechtswissenschaftlichen Schrifttum $^{38}$ findet sich dieser weitergehende Infrastrukturbegriff.

Die deutsche Gesetzessprache hingegen ist in dieser Hinsicht wenigstens klar, sie verwendet einen rein anlagenbezogenen Infrastrukturbegriff. Das AEG stellt dem „Erbringen von Eisenbahnverkehrsleistungen“ ausdrücklich den „Betrieb von Eisenbahninfrastrukturen“ gegenüber ( $\$ \S 1$ Abs. 1 und 2 Abs. 1) und definiert die Eisenbahninfrastruktur als die Betriebsanlagen der Eisenbahnen ( $₫ 2$ Abs. 3). Auch $\S 19$ b Abs. 3 Buchst. a) LuftVG und $\S 2$ Abs. 2 Nr. 3 ROG unterscheiden ausdrücklich zwischen „Dienstleistungen und Infrastrukturen“. § 28a Abs. 1 Nr. 3 EnWG geht davon aus, dass „die Infrastruktur“ Eigentum einer natürlichen oder juristischen Person sein kann. Das TKG bezieht in $\S 77$ a die ,gemeinsame Nutzung von Infrastrukturen" ausdrücklich auf Verkabelungen und Kabelkanäle, in $\S 77$ b sind mit ,alternativen Infrastrukturen“ ausschließ-

tenden energierechtlichen Ordnungsrahmens, vgl. resümierend J. H. Klement Die Städte, der Strom und das Gemeinwohl, FAZ v. 30.8. 2013, 12.

${ }^{37}$ So z.B. bei Frey (Fn. 12), 20.

${ }^{38}$ Hermes Infrastrukturverantwortung (Fn. 11), 172: sowohl die „Wegenetze“, als auch die mit ihrer Hilfe erbrachten „Dienste“. Für die Einbeziehung staatlicher Maßnahmen neben Einrichtungen Hünnekens (Fn. 11), 15 f., im Anschluß an K. Stern Staatsrecht I, 1984, § 21 II.2 und $R$. Stober Handbuch des Wirtschaftsverwaltungs- und Umweltrechts, 1989, § $47 \mathrm{I}$. 
lich „Einrichtungen“ gemeint ${ }^{39}$. Auch die EU-Richtlinien, die auf eine Liberalisierung mitgliedstaatlicher Infrastrukturdienste zielen und zum großen Teil den genannten deutschen Regelungen zugrunde liegen, verwenden einen anlagenbezogenen Infrastrukturbegriff. ${ }^{40}$ Der in Art. 170 Abs. 1 AEUV verwendete Infrastrukturbegriff ist sprachlich offen, wird in Praxis und Literatur allerdings nur auf die relevanten Einrichtungen bezogen, also ebenfalls anlagenbezogen verstanden. ${ }^{41}$

Anknüpfend an diese gesetzliche Vorformung liegt es nahe, den Rechtsbegriff „Infrastruktur“ nur auf die ortsfesten Anlagen und Einrichtungen zu beziehen, die der Versorgung der Allgemeinheit mit essentiellen Gütern oder Leistungen dienen. Die allgemeinnützigen Dienstleistungen, die mit Hilfe dieser Anlagen erbracht werden, sind dann Infrastrukturleistungen oder-dienste.

Charakteristikum vieler Infrastrukturen ist ihre Netzgebundenheit, die, wie z.B. Georg Hermes und Jürgen Kühling gezeigt haben, besondere Anforderungen an die rechtliche Analyse und Konstruktion stellt. Angesichts seiner weiterreichenden Funktion scheint es mir allerdings zu eng, den Rechtsbegriff der Infrastruktur von vornherein darauf zu beschränken $^{42}$ : Auch Einrichtungen der punktförmigen Infrastruktur ohne Netzanbindung (Schulen, Bibliotheken, Krankenhäuser, Altenheime, Friedhöfe) bilden eine immobile sächliche Grundlage staatlich verantworteter Versorgung und verlangen daher nach einer entsprechenden rechtlichen „Bearbeitung“.

\footnotetext{
${ }^{39}$ In begrifflicher Hinsicht verwirrend redundant dann allerdings die Formel von der „physischen Infrastruktur" in $\S 3$ Nr. 32 Buchst. b, Nr. 33b TKG.

${ }^{40}$ Vgl. z.B. Art. 3 und 6 RL 91/440/EWG zur Entwicklung der Eisenbahnunternehmen der Gemeinschaft; Art. 2 RL 2001/14/EG über die Zuweisung von Fahrwegkapazität der Eisenbahn und die Erhebung von Entgelten für die Nutzung von Eisenbahninfrastruktur; Art. 3 Nr. 3 RL 2012/34/EU zur Schaffung eines einheitlichen europäischen Eisenbahnraums (ABIEU 2012 L 343/32); Art. 36 Abs. 1 RL 2009/73/EG über gemeinsame Vorschriften für den Erdgasbinnenmarkt; Art. 2 Nr. 1 VO (EU) Nr. 347/2013 zu Leitlinien für die transeuropäische Energieinfrastruktur (ABIEU 2013 L 115/39).

${ }^{41}$ Vgl. z.B. H. Lecheler in: Grabitz/Hilf/Nettesheim, Recht der EU, Art. 170 AEUV Rn. 12-17; Chr. Calliess in: Calliess/Ruffert, EUV/AEUV, Art. 170 AEUV Rn. 7; Voet van Vormizeele in: Schwarze (Hrsg.) EU-Kommentar, Art. 170 AEUV Rn. 9-12; J. Kühling Sektorspezifische Regulierung in den Netzwirtschaften, 2004, 529. Ebenso Art. 2 Nr. 1 der auf Art. 172 AEUV gestützten VO (EU) Nr. 347/2013 zu Leitlinien für die transeuropäische Energieinfrastruktur (ABl. 2013 L 115/39): „Energieinfrastruktur“ bezeichnet jede materielle Ausrüstung oder Anlage, die ...".

${ }^{42}$ So aber Hermes Infrastrukturverantwortung (Fn. 11), 329: alle Mittel, die der Überwindung von Entfernung dienen und dadurch die Integration eines Raumes bewirken. Gegen diese begriffliche Beschränkung auch Kühling Regulierung (Fn. 41), 45; ders. Anforderungen an ein zukunftsfähiges Infrastrukturrecht, DVBl 2013, 1093 (1094).
} 
„Infrastrukturrecht“ beschreibt dann die Regeln über die Planung, Errichtung, den Unterhalt und den Betrieb von Infrastruktur. Es handelt sich auch nach Jahren des juristischen - vor allem regulierungsrechtlichen - Diskurses nicht um ein eigenständiges, klar abgrenzbares Rechtsgebiet. Zwar finden sich wegen der ihnen gemeinsamen Vorformung durch das Unionsrecht in einigen Umsetzungsgesetzen ähnliche Regelungsmuster, zum Teil gleichlautende Begrifflichkeiten. Eine dogmatisch stringente Durchformung, die trotz unterschiedlicher Gegenstände auf gemeinsame und doch spezielle Rechtsgrundsätze hinwiese und das „Infrastrukturrecht“ von anderen Bereichen des Verwaltungsrechts abhöbe, ist hingegen nicht erkennbar. ${ }^{43}$ Statt dessen handelt es sich um eine deskriptive Sammelbezeichnung für eine Teilmenge des Rechts der Daseinsvorsorge, deren Regeln verschiedenen, überwiegend spezialgesetzlich kodifizierten Teilrechtsgebieten ${ }^{44}$ entstammen. Infrastrukturrecht ist eine verkürzende Chiffre für jene - disparaten - Teile der Rechtsordnung, die sich auf ortsfeste Anlagen mit einem staatlich verantworteten Versorgungszweck beziehen.

\section{Infrastruktur und Daseinsvorsorge}

Der Begriff der Infrastruktur hat im deutschen Rechtsdiskurs unweigerlich einen anderen terminologischen Klassiker im Schlepptau, die „Daseinsvorsorge“. Diesen als Begriff und Konzept darzustellen, gar auszuleuchten, verbietet sich in diesem Kreis, zu oft beschrieben und erörtert ist die Forsthoff'sche Wortschöpfung ${ }^{45}$, die sich seit dieser Grundlegung

${ }^{43}$ Für ein „Querschnittsrechtsgebiet im Werden“ hingegen Kühling (Fn. 42), DVB1 2013, 1093 (1094). Die von Heiko Faber eingeführte Kategorisierung der „Infrastrukturverwaltung “ als eigenständigen Bereich des Verwaltungshandelns (Verwaltungsrecht, 4. Aufl.1995, § 5 II und $\S \S 31,32$ ) hat sich nicht durchgesetzt.

${ }_{44}$ Wie z.B. dem Abfallrecht, Bau- und Planungsrecht, Eisenbahnrecht, Energierecht, Kommunalrecht, Luftverkehrsrecht, Personenbeförderungsrecht, Postrecht, Schul- und Hochschulrecht, Sozialrecht, Straßen- und Wasserstraßenrecht, Subventionsrecht, Wasserrecht, Telekommunikationsrecht, Vergaberecht.

${ }^{45}$ Grundlegend E. Forsthoff Die Verwaltung als Leistungsträger, 1938; ders., Rechtsfragen der leistenden Verwaltung, 1959. Zusammenfassungen z.B. bei $W$. Löwer Energieversorgung zwischen Staat, Gemeinde und Wirtschaft, 1989, 110-117; Hermes Infrastrukturverantwortung (Fn. 11), 94-100; M. Knauff Der Gewährleistungsstaat: Reform der Daseinsvorsorge, 2004, 38-43; M. Krajewski Grundstrukturen des Rechts öffentlicher Dienstleistungen, 2011, 15-26. Zum geistigen Umfeld z.B. M. Stolleis Geschichte des öffentlichen Rechts in Deutschland, Bd. III, 1999, 366-368 und Bd. IV, 2012, 262-265; J. Kersten Die Entwicklung des Konzepts der Daseinsvorsorge im Werk von Ernst Forsthoff, Der Staat 44 (2005), 543 (544-554). 
bekanntlich fortentwickelt hat ${ }^{46}$ und dabei nicht nur dem demokratischen Ordnungsrahmen des Grundgesetzes angepasst wurde. Die Debatte um Privatisierung und Privatisierungsfolgenrecht der 1990er Jahre bewirkte (nicht zuletzt unter dem Einfluss des europäischen Binnenmarktrechts) weitere entscheidende Verschiebungen: Implizierte noch bei Forsthoff das klassische Konzept der Daseinsvorsorge die Selbstvornahme des Staates und schloss jede private Leistungserbringung, erst recht privaten Wettbewerb aus ${ }^{47}$, so ist die Beteiligung Privater an der staatlichen Daseinsvorsorge heute tatsächlich Allgemeingut und rechtsdogmatisch vielfach dargestellt und erörtert. ${ }^{48}$ Auch wenn die Daseinsvorsorge - als Begriff wie als Konzept - schon häufig für überholt erklärt wurde, und zwar gerade im Hinblick auf eine liberalisierte Versorgungswirtschaft mit staatlicher Residualverantwortung, ${ }^{49}$ so haben doch Begriffsvarianten, wie z.B. „öffentliche Versorgung "50 oder „öffentliche Dienstleistungen "51, bislang den Forsthoff'schen Begriff nicht verdrängen können. Dieser hat sich vielleicht auch deshalb als so wirkmächtig erwiesen, weil darin sowohl das wohlfahrtsstaatliche als auch das existentielle Element sprachlich prägnant zum Ausdruck kommen. Ich meine, auch in der modernen, von Verantwortungstypologien geprägten Rechtsdogmatik hat die Daseinsvorsorge daher ihren Platz. ${ }^{52}$

${ }^{46}$ Hierzu z.B. Kersten (Fn. 45) Staat 44 (2005), 543 (555-564); Krajewski Grundstrukturen (Fn. 45), 21-26.

${ }^{47}$ Vgl. Forsthoff Rechtsfragen (Fn. 45), 11; ebenso z.B. H. H. Klein Die Teilnahme des Staates am wirtschaftlichen Wettbewerb (1968), 18. Daher sei alle Daseinsvorsorge öffentliche Verwaltung, vgl. Forsthoff Verwaltungsrecht I, 10. Aufl. 1973, 370. Allerdings konnte Forsthoff sich vorstellen, dass sich der Staat in der Daseinsvorsorge aus der Leistungserbringung zugunsten ,unterstaatlicher, gemeinschaftsförmiger Leistungsträger" (gemeint waren vor allem die Gemeinden) zurückzieht und sich auf eine „Oberaufsicht" über diese beschränkt, vgl. Verwaltung (Fn. 45), 49 f.

48 Vgl. statt aller $R$. Schmidt Die Liberalisierung der Daseinsvorsorge, Der Staat 42 (2003), 225 (242 f.); M. Ronellenfitsch in: Magiera/Sommermann (Hrsg.) Daseinsvorsorge und Infrastrukturgewährleistung, 2009, 27 (32 f.).

49 So z.B. Glöckner Infrastrukturverantwortung (Fn. 11), 22-24; S. Boysen/M. Neukirchen Europäisches Beihilferecht und mitgliedstaatliche Daseinsvorsorge, 2007, 23.

${ }^{50}$ J.-Chr. Pielow Grundstrukturen öffentlicher Versorgung, 2001, 10-18; H. Butzer Sicherstellungsauftrag, in: Isensee/Kirchof (Hrsg.) HStR IV, 3. Aufl. 2006, § 74 Rn. 24-29.

${ }^{51}$ Statt aller Krajewski Grundstrukturen (Fn. 45), 3-14. Austauschbar mit Daseinsvorsorge offensichtlich in $\S 2$ Abs. 1 Nr. 2 UIG verwendet. Aus dem Unionsbereich z.B. die Kommissionsmitteilungen zu Ausgleichsleistungen für die „Erbringung öffentlicher Dienstleistungen“ in ABIEU 2005 C 297/4 und ABIEU 2012 C 8/15.

52 Zur Vereinbarkeit der Konzepte Daseinsvorsorge und Gewährleistungsverantwortung z.B. P. Badura Wirtschaftliche Betätigung der öffentlichen Hand zur Gewährleistung von Daseinsvorsorge, in: J. Schwarze (Hrsg.) Daseinsvorsorge im Lichte des 
Als rechtstatsächliche Beschreibung geläufig, als deskriptiver Rechtsund Gesetzesbegriff in Verwendung ${ }^{53}$, ist die Vokabel „Daseinsvorsorge“ also mit gutem Grund fester Bestandteil jedes - juristischen oder anderen - Diskurses über öffentliche Aufgaben und ihre Wahrnehmung. Er bezeichnet die grundlegende Aufgabe des sozialen Rechtsstaates, für die Versorgung der Bevölkerung mit den im modernen Alltag unverzichtbaren Gütern und Dienstleistungen zu sorgen. Zur Kennzeichnung von Reichweite und Intensität dieser Aufgabe spricht das BVerfG von Leistungen, derer der Bürger ,zur Sicherung einer menschenwürdigen Existenz unumgänglich bedarf“ $54, \S 2$ Abs. 2 Nr. 3 ROG von der „Grundversorgung für alle Bevölkerungsgruppen“. Im Übrigen beschreibt der deutsche Gesetzgeber die Schwelle der staatlichen Verantwortung für die Daseinsvorsorge mit dem Instrument des Universaldienstes in regulierten Leistungsbereichen: Dabei handelt es sich um solche „Dienstleistungen, die allgemein als unabdingbar angesehen werden“ ( $§ 11$ Abs. 1 S. 3 PostG) bzw. „deren Erbringung für die Öffentlichkeit als Grundversorgung unabdingbar geworden ist" ( $§ 78$ Abs. 1 TKG). Diese Formulierungen belegen schon, dass der Aufgabenbereich der Daseinsvorsorge nicht dauerhaft fixiert oder durch einen abschließenden Katalog zu bestimmen, sondern notwendigerweise entwicklungsoffen ist: ${ }^{55}$ Vor allem ist er von der sozialen und

Wettbewerbsrechts, 2001, 25 (27); M. Möstl Renaissance und Rekonstruktion des Daseinsvorsorgebegriffs unter dem Europarecht, FS Badura, 2004, 951 (959 f.); Knauff Gewährleistungsstaat (Fn. 45), 91; R. Uerpmann-Wittzack Verkehr, in: HStR IV (Fn. 50), § 89 Rn. 1; Kotzur (Fn. 28), JöR n.F. 61 (2013), 195 (200 f.). Für eine „Daseinsvorsorgeverantwortung“ z.B. M. Möstl in: Maunz/Dürig, GG, Art. 87e Rn. 138 f. (2006).

53 Vgl. z.B. § 4 Abs. 1 S. 3, § 25 Abs. 2 PostVerfG 1989 (BGBl. I 1026); § 8 Abs. 1 G über die Regulierung der Telekommunikation und des Postwesens 1994 (BGBl. I 2371); $\S 1$ Abs. 1 Gesetz zur Regionalisierung des ÖPNV (RegG); § 2 Abs. 2 Nr. 1 und 3 ROG; $\S 2$ Abs. 1 Nr. 2 UIG; $\S 50$ Abs. 1 WHG; weitere Nachw. bei Krajewski Grundstrukturen (Fn. 45), 40-42. Aus dem Landesrecht z.B. § 78c S. 2 Nr. 2 BayVwVfG. Aus der Rspr. statt aller BVerfGE 22, 28 (38); 66, 248 (258); 75, 192 (197-200); 107, 59 (93); 128, 226 (250); 130, 76 (115); BVerfG NJW 1990, 1783; als „Daseinssorge“ (sic !) in BVerfGE 38, 258 (270). Gegen „Daseinsvorsorge“ als Rechtsbegriff z.B. Schmidt (Fn. 48), Der Staat 42 (2003), 225 (229 f. und 247); skeptisch M. Krajewski Rechtsbegriff Daseinsvorsorge?, VerwArch 99 (2008), 174.

${ }^{54}$ BVerfGE 66, 248 (258). Forsthoff hatte ganz in diesem Sinne zunächst auf die „lebenswichtige Angewiesenheit“ des Einzelnen, seine „Vorsorgebedürftigkeit“ abgestellt, vgl. Verwaltung (Fn. 45), 42: Bäder und Heilbäder sollten umfasst sein, Theater und „Lichtspiele" hingegen nicht; deutlich weiter dann schon Forsthoff Rechtsfragen (Fn. 45), 12, wo alle für die Allgemeinheit erbrachten und grundsätzlich zugänglichen Verwaltungsleistungen erfasst sein sollten.

55 Ebenso schon Forsthoff Rechtsfragen (Fn. 45), 12. Vgl. auch $\S 11$ Abs. 2 S. 2 PostG, wonach die als „unabdingbar“ geltenden Leistungen der Universaldienstver- 
technischen Entwicklung abhängig, unter deren Einfluss sich die Grundund Versorgungsbedürfnisse der Allgemeinheit sowie die technischen Möglichkeiten ihrer Befriedigung permanent verändern. Das überkommene Konzept der Daseinsvorsorge ist in seiner modernen Gestalt grundsätzlich flexibel genug, um diese Änderungen zu verarbeiten.

Aus alldem ergibt sich ein modales Verhältnis von Infrastruktur zur Daseinsvorsorge: Letztere bezeichnet die öffentliche Aufgabe und die zu ihrer Erfüllung ins Werk gesetzte Tätigkeit, "Infrastruktur" hingegen einen Gegenstand und ein wesentliches Instrument zur Erfüllung dieser Aufgabe, nämlich ihre immobilen sächlichen Grundlagen. Infrastruktur ist ein unabdingbares Mittel, um die Aufgabe der Daseinsvorsorge zu erfüllen; ${ }^{56}$ dementsprechend gibt es, wie $\S 2$ Abs. 2 Nr. 3 ROG formuliert, "Infrastrukturen der Daseinsvorsorge“. Wie das Konzept der Daseinsvorsorge, ist auch der Infrastrukturbegriff grundsätzlich entwicklungsoffen: Ändern sich die Grund- und Versorgungsbedürfnisse der Allgemeinheit und die technische Möglichkeiten ihrer Befriedigung, so kann sich auch die Palette der „Hardware“ ändern, die hierfür gebraucht wird.

„Infrastrukturrecht" besitzt dementsprechend im Kern einen instrumentellen Charakter, es ist stets auf die Erfüllung der durch Verfassung oder Gesetz determinierten Versorgungszwecke bezogen und in der Regel beschränkt.57 Gleichzeitig ergibt sich, dass „Infrastrukturrecht“ nicht vom Recht der Daseinsvorsorge getrennt werden kann, Debatten über dieses betreffen fast zwingend stets auch das Recht der Infrastruktur.

\section{Europäische Konzepte}

Im europäischen Unionsrecht findet die Daseinsvorsorge ${ }^{58}$ eine Entsprechung in einem etwas umständlichen Begriffspaar, den „Diensten von

pflichtung „,er technischen und gesellschaftlichen Entwicklung nachfragegerecht anzupassen" sind.

${ }^{56}$ Dies wird auch im Wortlaut der ersten deutschen Gesetze deutlich, die beide Begriffe zugleich verwendeten, vgl. z.B. § 4 Abs. 1 S. 3 PostVerfG 1989 (BGBl. I 1026): Infrastrukturdienste und notwendige Infrastruktur ,sind im Sinne der öffentlichen Aufgabenstellung, insbes. der Daseinsvorsorge, ... zu sichern und der Entwicklung anzupassen"; nach Art. 25 Abs. 2 PostVerfG war die Bundesregierung ermächtigt, durch Rechtsverordnung ,diejenigen Infrastrukturleistungen zu bestimmen, die die Unternehmen im besonderen öffentlichen Interesse, vor allem aus Gründen der Daseinsvorsorge erbringen müssen“; ebenso in $\S 8$ Abs. $1 \mathrm{G}$ über die Regulierung der Telekommunikation und des Postwesens 1994 (BGBl. I 2371).

${ }^{57}$ Erweiterungen der Zweckrichtung ergeben sich allerdings durch den Liberalisierungs- und Wettbewerbsimpuls des EU-Rechts, dazu unten III.4.

${ }^{58}$ Dieser Begriff selbst fand in den deutschen Fassungen einiger Mitteilungen der Kommission ausdrückliche Verwendung, vgl. nur ABIEG 1996 C 281/3; ABIEG 2001 C $17 / 4$. 
allgemeinem wirtschaftlichen Interesse“ (DAWIs) und den „Diensten von allgemeinem Interesse". ${ }^{59}$ Dabei handelt es sich um markt- bzw. nicht marktbezogene Tätigkeiten, die im Interesse der Allgemeinheit erbracht und daher von den Behörden mit spezifischen Gemeinwohlverpflichtungen verknüpft werden. ${ }^{60}$ Sie werden in Art. 14 AEUV ${ }^{61}$ der gemeinsamen Sorge von Union und Mitgliedstaaten überantwortet. Gleichzeitig verspricht Art. 106 Abs. 2 AEUV Unternehmen, die mit der Erbringung derartiger Dienste betraut sind, unter bestimmten Voraussetzungen Abweichungen vom Wettbewerbsrecht des europäischen Binnenmarktes. Leistungen der Daseinsvorsorge erscheinen im Unionsrecht also einerseits in der Rolle der rechtfertigungsbedürftigen Wettbewerbseinschränkung, andererseits aber als ein eigenständiger, positiver Rechtswert, dessen Bedeutung für den ,sozialen und territorialen Zusammenhalt“ Art. 14 AEUV ausdrücklich betont. ${ }^{62}$ Die Werthaftigkeit der DAWIs unterstreicht seit dem Vertrag von Lissabon zusätzlich ein separates Protokoll ${ }^{63}$, dessen Art. 1 nicht nur die Autonomie der staatlichen Behörden und die notwendige Vielfalt der erfassten Leistungen unterstreicht, sondern auch bestimmte Erbringungsparameter formuliert ${ }^{64}$. Diese unions-

59 Vgl. für das Nebeneinander beider Begriffe nur Art. 1 und 2 des ,Protokolls über Dienste von allgemeinem Interesse“, ABlEU 2012 C 326/308. Zur Begriffsdoppelung grundlegend die Mitteilung der Kommission über ,die Leistungen der Daseinsvorsorge in Europa“ (ABlEG 2001 C 17 Rn. 27-30); näher Krajewski Grundstrukturen (Fn. 45), 77-115. Die terminologische Vielfalt des Unionsrechts ist damit allerdings noch nicht erschöpft, denn Art. 93 AEUV, der bestimmte Verkehrsbeihilfen vom Beihilfeverbot ausnimmt, bezieht sich hierfür auf „,mit dem Begriff des öffentlichen Dienstes zusammenhängende Leistungen“; konkretisiert wird diese Regelung durch die VO 1370/2007 über öffentliche Personenverkehrsdienste auf Schiene und Straße, deren Art. 1 Abs. 1 wiederum von „Dienstleistungen von allgemeinem Interesse“ spricht.

60 Vgl. z.B. die Kommissionsmitteilungen ,Leistungen der Daseinsvorsorge in Europa" (Fn. 58) sowie das Grünbuch der Kommission zu Dienstleistungen von allgemeinem Interesse v. 21.5.2003, KOM(2003) 270 endg, Tz. 16-17. Zum Konzept statt vieler Th. v. Danwitz Dienste von allgemeinem wirtschaftlichen Interesse in der europäischen Wettbewerbsordnung, in: Krautscheid (Hrsg.) Die Daseinsvorsorge im Spannungsfeld von europäischem Wettbewerb und Gemeinwohl, 2009, 103 (113-117).

61 Dessen Regelungsgegenstand in BVerfGE 123, 267 (294) umstandslos mit „Daseinsvorsorge" bezeichnet wird.

62 Für ein „Unionsstrukturprinzip“ Kotzur (Fn. 28), JöR n.F. 61 (2013), 195 (205). In Art. 36 EU-Grundrechtecharta wird dies wiederholt und nur scheinbar ergänzt um eine subjektiv-rechtliche Komponente: Die Union ,anerkennt und achtet" danach den Zugang zu DAWIs nach Maßgabe einzelstaatlicher Regelungen.

63 S. in Fn. 59.

64 Wie Qualität, Sicherheit, Bezahlbarkeit, Gleichbehandlung, universeller Zugang und Nutzerrechte. Zur prinzipienbildenden Rolle dieser Anforderungen z.B. M. Krajew$s k i$ Dienstleistungen von allgemeinem Interesse als Element europäischer Sozialstaat- 
rechtliche Verankerung der Daseinsvorsorge schließt als deren wesentliches Instrument die Infrastruktur mit ein.

\section{Die Infrastrukturverantwortung des Staates}

$\mathrm{Zu}$ den Anforderungen an ein „,zukunftsfähiges Infrastrukturrecht“ gehört, dass seine Ausgestaltung es dem Staat ermöglicht, seine ihm durch die Verfassung aufgegebene Verantwortung für Errichtung, Erhalt und Funktionsfähigkeit der für die Grundversorgung notwendigen Infrastruktur wahrzunehmen. Hierfür bedarf es geeigneter Regelungs- und Organisationsstrukturen für die Infrastruktur selbst sowie eines angemessenen rechtlichen Ordnungsrahmens für die Erbringung von Infrastrukturleistungen. „Infrastrukturrecht“ muss nicht nur einen verlässlichen Rahmen für die Erbringung dieser Leistungen bieten, sondern gleichzeitig - und vielleicht vorrangig - die Erfüllung des infrastrukturbasierten Daseinsvorsorgeauftrags des Staates gewährleisten.

\section{Verfassungsrechtliche Grundlagen}

Von Herbert Krüger wissen wir, dass ein öffentliches Straßen- und Wegenetz eine notwendige Voraussetzung staatlicher Existenz ist. ${ }^{65}$ Die Erschließung des Staatsgebiets gehört daher wie die Bereitstellung einer Rechtsordnung zu den notwendigen Staatsaufgaben. ${ }^{66}$ Von dort ist es nur ein kleiner Schritt, um zu erkennen, dass Infrastruktur, bezogen auf ein Mindestmaß an Versorgung, Mobilität und Kommunikation, eine Grundbedingung für die politische Integration jedes Gemeinwesens, zumal des Staates ist. ${ }^{67}$ Gerade in Zeiten einer schrumpfenden Bevölkerung ${ }^{68}$ sichern

lichkeit, in: J. Bast/F. Rödl (Hrsg.) Wohlfahrtsstaatlichkeit und soziale Demokratie in der Europäischen Union, EuR Beiheft 1/2013, 109 (117-120).

${ }^{65}$ H. Krüger Gegen eine Entstaatlichung der öffentlichen Wege, 1954, 36-38; ders. Marktwirtschaftliche Ordnung und öffentliche Vorhaltung der Verkehrswege, 1969, 4-8.

${ }^{66}$ J. Isensee Staatsaufgaben, in: HStR IV (Fn. 50), § 73 Rn. 30; U. Mager in: Säcker (Hrsg.) TKG. Kommentar, 3. Aufl. 2013, vor $\$ 78$ Rn. 2.

${ }^{67} \mathrm{Im}$ Anschluss an Krüger z.B. M. Fehling Mitbenutzungsrechte Dritter bei Schienenwegen, Energieversorgungs- und Telekommunikationsleitungen vor dem Hintergrund staatlicher Infrastrukturverantwortung, AöR 121 (1996), 59 (80 f.); Hermes Infrastrukturverantwortung (Fn. 11), 324-327; Schoch (Fn. 22), 198, in Fn. 192.

${ }^{68} \mathrm{Zu}$ diesem Aspekt im vorliegenden Zusammenhang insbes. J. Kersten Universaldienste in einer schrumpfenden Gesellschaft, DVB1 2006, 942; ders. Mindestgewährleistungen im Infrastrukturrecht, IzR 2008, 1 (2-3); F. Brosius-Gersdorf Demografischer Wandel und Daseinsvorsorge, VerwArch 98 (2007), 317 (319-324). 
vernetzte Infrastrukturen den sozialen Zusammenhalt von Staat und Gesellschaft. Diese Integrationsfunktion kann in Letztverantwortung nur der Verfassungsstaat erfüllen, sie obliegt ihm daher kraft seiner Existenz. Die Sicherstellung der für das Gemeinwesen notwendigen Infrastruktur ist somit eine immanente Kernfunktion des Staates und von Verfassungs wegen wesentlicher Gegenstand seiner Steuerungsverantwortung. ${ }^{69}$

In der geschriebenen Verfassung finden sich vor allem zwei Anknüpfungspunkte für einen umfassenden Infrastrukturauftrag des Staates. Zum einen ist er im Sinne einer Grundversorgung mit elementaren Gütern aus dem Sozialstaatsprinzip abzuleiten, verpflichtet dieses doch den Staat des Grundgesetzes dazu, für die Mindestvoraussetzungen eines menschenwürdigen Lebens seiner Bevölkerung zu sorgen. ${ }^{70}$ Dies schließt die Sicherung zeitgemäßer sozialer Existenzbedingungen ein. ${ }^{71}$ Vorbehaltlich eines großen Ausgestaltungsspielraums ist der Sozialstaat also verpflichtet, grundsätzlich für eine dauerhafte, flächendeckende und sozialverträgliche Grundversorgung mit Infrastruktur und Infrastrukturleistungen zu sorgen. ${ }^{72}$ Im Lichte des Sozialstaatsprinzips umfasst die Aufgabe

${ }^{69}$ Statt vieler Osterloh Privatisierung (Fn. 6), 208 und 229; Hermes Infrastrukturverantwortung (Fn. 11), 147, 152-156. Für die Daseinsvorsorge ähnlich W. Rüfner Daseinsvorsorge und soziale Sicherheit, in: HStR IV (Fn. 50), § 96 Rn. 1 und 21; H.-P. Bull Daseinsvorsorge im Wandel der Staatsformen, Der Staat 47 (2008), 1 (9); als ,essentielle Staatsfunktion“ bezeichnet von Lübbe-Wolff in abw. Meinung, BVerfGE 118, 124 (151). Auch der Ahnherr der Wirtschaftstheorie, Adam Smith, zählte die Errichtung und den Unterhalt nützlicher „öffentlicher Anlagen und Einrichtungen“ zu den drei Kernaufgaben des Staates, vgl. An Inquiry into the Nature and Causes of the Wealth of Nations (1776), Book V, Chapter I, Part III, zitiert nach der Ausgabe The Modern Library, New York, 1937. Die Untiefen einer Staatsaufgabenlehre betonen im vorliegenden Zusammenhang z.B. Hermes Infrastrukturverantwortung (Fn. 11), 135-139; J. A. Kämmerer Privatisierung, 2001, 157-163; Kühling Regulierung (Fn. 41), 446-450; O. Lepsius in: Fehling/Ruffert (Fn. 20), § 4 Rn. 101-103; allgemein auch Isensee (Fn. 66), § 73 Rn. 1 („Entwicklungsland der deutschen Staatsrechtslehre“).

${ }^{70}$ StRspr., z.B. BVerfGE 40, 121 (133); 82, 60 (80, 85); 110, 412 (445 f.); ebenso z.B. I. Spiecker gen. Döhmann Verfassungstheorie des Sozialstaates, in: Depenheuer/Grabenwarter (Hrsg.) Verfassungstheorie, 2010, § 23 Rn. 47 f. Für die Ausrichtung von Daseinsvorsorge und Infrastruktur auf die Gewährleistung sozialer Gleichheit hingegen H. F. Zacher Das soziale Staatsziel, in: Isensee/Kirchhof (Hrsg.) HStR II, 3. Aufl. 2004, $\S 28 \mathrm{Rn} .36$.

${ }^{71}$ R. Gröschner in: Dreier, GG, 2. Aufl. 2006, Art. 20 Rn. 55; Kämmerer Privatisierung (Fn. 69), 430.

72 Bauer Privatisierung (Fn. 6), 269 mwN; Osterloh Privatisierung (Fn. 6), 208; Fehling (Fn. 67), AöR 121 (1996), 59 (81 f.); Kämmerer Privatisierung (Fn. 69), 430; Hünnekens (Fn. 11), 116-123; Butzer (Fn. 50), § 74 Rn. 44; Glöckner Infrastrukturverantwortung (Fn. 11), 63 mwN; J. Brehme Privatisierung und Regulierung der öffentlichen Wasserversorgung, 2010, 328-330. Ebenso für die Daseinsvorsorge statt aller Zacher 
der Daseinsvorsorge dabei nicht nur die Versorgungssicherheit, sondern auch die tatsächliche Erreichbarkeit der essentiellen Güter: Die Versorgungsleistungen, für die der Staat verantwortlich ist, müssen für die Allgemeinheit erschwinglich ${ }^{73}$, die Einrichtungen, in denen die Leistungen erbracht werden, jedenfalls grundsätzlich allgemein zugänglich sein.

Mit dem sozialstaatlichen Gestaltungsauftrag verbunden sind die grundrechtlichen Schutzpflichten für Menschenwürde, Leben und Gesundheit, die sich zu einem Verfassungsauftrag für Infrastrukturvorsorge verdich$\operatorname{ten}^{74}$. Auch andere Grundrechte sind auf infrastrukturelle Voraussetzungen angewiesen: Ohne Teilhabe des Bürgers an Netzen und Leistungen der Infrastruktur ist seine gesellschaftliche Integration und die Verwirklichung seiner verfassungsrechtlich garantierten Freiheiten von Meinungsäußerung, Information, Versammlung, Fortbewegung, Beruf etc. praktisch nicht denkbar. Die staatliche Aufgabe des „Grundrechtsvoraussetzungsschutzes "75 verpflichtet den Staat daher zu aktiver Infrastrukturpolitik. Ähnlich folgt aus Art. 7 Abs. 1 GG die staatliche Verpflichtung, ein leistungsfähiges Schulsystem zu gewährleisten. ${ }^{76}$

Ist die prinzipielle verfassungsrechtliche Verankerung auch wenig bestritten, so ist die Direktionskraft des Sozialstaatsprinzips und der Grundrechte in diesem Zusammenhang doch begrenzt: Beide verpflichten nicht zu bestimmten sozialen Leistungen oder Maßnahmen ${ }^{77}$, sie enthalten keine Aussagen zum Versorgungs- und Vorsorgeumfang oder zum Versorgungsmodus. Der weite Gestaltungsspielraum des Staates ist prägend für beide Ansatzpunkte der verfassungsrechtlichen Infrastruktur-

(Fn. 70), § 28 Rn. 64-67; Knauff Gewährleistungsstaat (Fn. 45), 192-195; Lepsius (Fn. 69), § 4 Rn. 97.

73 Kämmerer Privatisierung (Fn. 69), 430; Ronellenfitsch (Fn. 48), 34; H.-G. Henneke Die Daseinsvorsorge in Deutschland - Begriff, historische Entwicklung, rechtliche Grundlagen und Organisation, in: Krautscheid, Daseinsvorsorge (Fn. 60), 17 (19).

${ }^{74}$ Zur Relevanz der Grundrechte für die Gewährleistung der Grundbedingungen menschlicher Existenz z.B. Bauer Privatisierung (Fn. 6), 269; Kämmerer Privatisierung (Fn. 69), 449-459; Knauff Gewährleistungsstaat (Fn. 45), 188-192; Schoch, NVwZ 2008, 242 (244); Kersten (Fn. 68), IzR 2008, 1 (11); Glöckner Infrastrukturverantwortung (Fn. 11), $66 \mathrm{f}$.

75 Schoch (Fn. 22), 187 f.; ausführlich W. Weiß Privatisierung und Staatsaufgaben, 2002, 147-205.

${ }^{76}$ Kersten (Fn. 68), IzR 2008, 1 (10); J. Ennuschat Organisation der öffentlichen Schule, Verw. 45 (2012), 331 (336 f.); Chr. Langenfeld in: Dörr/Grote/Marauhn (Hrsg.) Konkordanzkommentar EMRK/GG, 2. Aufl. 2013, Kap. 23 Rn. 10.

${ }_{77}$ Für das Sozialstaatsprinzip statt aller BVerfGE 82, 60 (80); 94, 241 (263); 110, 412 (445). Für die grundrechtlichen Schutzpflichten z.B. BVerfGE 77, 170 (214 f.); 79, 174 (202); 115, 25 (43-45); B. Wollenschläger Effektive staatliche Rückholoptionen bei gesellschaftlicher Schlechterfüllung, 2006, 83-94. 
pflicht. ${ }^{78}$ Griffiger sind da schon die konkreten sozialstaatlichen Ausprägungen im Verfassungstext, wie vor allem die expliziten Gewährleistungsaufträge in Art. 87e Abs. 4 und 87f Abs. 1 GG für die Verkehrsbedürfnisse der Allgemeinheit im Eisenbahnverkehr bzw. für „flächendeckend angemessene und ausreichende Dienstleistungen“ im Bereich Post und Telekommunikation. ${ }^{79}$

Auf unionsrechtlicher Ebene besteht ein Pendant in Art. 14 AEUV, der als Funktionsgarantie mit Schutzpflichtcharakter $\mathrm{zu}$ verstehen ist und damit einen Auftrag an Union und Mitgliedstaaten enthält, für das Funktionieren der innerstaatlichen Daseinsvorsorge zu sorgen. ${ }^{80}$ Dies umfasst die Verantwortung für das Vorhandensein und die Funktionsfähigkeit der notwendigen Infrastruktur. Die unionsrechtliche Grundsatznorm verstärkt damit nicht nur den verfassungsstaatlichen Auftrag und fügt ihm einen äußeren Impuls hinzu, sie formuliert - erst recht in Verbindung mit dem dazu angenommenen Protokoll ${ }^{81}$ - den öffentlichen Versorgungsauftrag sehr viel klarer und differenzierter als das Grundgesetz.

\section{Infrastruktur als Gegenstand von Gewährleistungsverantwortung}

Der infrastrukturbezogene Verfassungsauftrag des Staates ruft die verschiedenen Wahrnehmungsmodi staatlichen Handelns auf, die durch jenen nicht notwendigerweise determiniert sind. Sie reichen von der traditionellen staatlichen Leistungsgewährung als Selbstvornahme über die Indienstnahme oder Beleihung Privater, die vertragliche Kooperation mit

\footnotetext{
${ }_{78}$ Vgl. nur Voßkuhle Beteiligung Privater (Fn. 7), 297 f.; Hellermann Schutz (Fn. 8), 375 .

79 Zur sozialstaatlichen Grundierung dieser Vorschriften z.B. M. Cornils Staatliche Infrastrukturverantwortung und kontingente Marktvoraussetzungen, AöR 131 (2006), 378 (385); Knauff Gewährleistungsstaat (Fn. 45), 247; Möstl in: Maunz/Dürig, GG, Art. 87f Rn. 71 (2010). Interessanterweise ist im Verfassungstext der Gewährleistungsauftrag für Eisenbahnen auf Schienennetz und Verkehrsangebot gleichermaßen bezogen, schließt die Infrastruktur selbst also ein, während der Auftrag für Post und Telekommunikation nur die zu erbringenden Dienstleistungen nennt. Nach ganz h.M. aber ist die Sorge um das Netz, also um die Infrastruktur im eigentlichen Sinne, von letzterem mit umfasst, vgl. nur Fehling (Fn. 67), AöR 1996, 59 (78 f.); Hermes Infrastrukturverantwortung (Fn. 11), 239; Schoch (Fn. 22), 198, in Fn. 195; Möstl in: Maunz/Dürig, GG, Art. 87f Rn. 33 (2010).

${ }^{80} \mathrm{Vgl}$. St. Wernicke in: Grabitz/Hilf/Nettesheim, Recht der EU, Art. 14 EUV Rn. 39-49 („Funktionsgarantie und Optimierungsgebot“); Chr. Jung in: Calliess/Ruffert, EUV/AEUV, 4. Aufl. 2011, Art. 14 AEUV Rn. 22 („Funktionsgewährleistungspflicht“); Schmidt (Fn. 48), Staat 42 (2003), 225 (239: „Bestandsgarantie“); v. Danwitz Dienste (Fn. 60), $118 \mathrm{f}$.

${ }^{81}$ Dazu oben bei Fn. 63-64.
} 
ihnen bis zur bloßen Rahmensetzung. ${ }^{82}$ Die Tatsache, dass grundsätzlich verschiedene solcher Modi zur Verfügung stehen, bestimmt die insoweit dem Staat von Verfassungs wegen zukommende Rolle, die seit geraumer Zeit mit verschiedenen Verantwortungsschichten und -konzepten ${ }^{83}$ beschrieben wird.

Für die Wahrnehmung der Daseinsvorsorge haben sich seit Mitte der 1990er Jahre Begriff und Konzept der „Gewährleistungsverantwortung“ etabliert $^{84}$, wofür man begrifflich an entsprechende Formulierungen im Grundgesetz (Art. 87e Abs. 4, Art. 87f Abs. 1) anknüpfen konnte. Danach muss der Staat die ihm von Verfassungs wegen zufallende Aufgabe nicht mehr selbst erfüllen, er muss für ihre Erfüllung einstehen. Soweit

${ }^{82}$ Hierzu umfassend H. Schulze-Fielitz Grundmodi der Aufgabenwahrnehmung, in: Hoffmann-Riem/Schmidt-Aßmann/Voßkuhle (Hrsg.) GVwR, Bd. I, 2. Aufl. 2012, § 12.

${ }^{83}$ Zum schillernden Begriff der Verantwortung die Hinweise bei Voßkuhle Beteiligung Privater (Fn. 7), 270, in Fn. 9 und 10; Franzius Gewährleistung (Fn. 29), 96-102 sowie umfassend $J$. H. Klement Verantwortung, 2006. Zu den verschiedenen Verantwortungstypologien z.B. Voßkuhle Beteiligung Privater (Fn. 7), in Fn. 65; Bauer Privatisierung (Fn. 6), 278-280; Butzer (Fn. 50), § 74 Rn. 12; Brehme Privatisierung (Fn. 72), 46-53; grundlegend E. Schmidt-Aßmann in: Hoffmann-Riem/Schmidt-Aßmann/Schuppert (Hrsg.) Reform des Allgemeinen Verwaltungsrechts - Grundfragen, 1993, 11 (43f.).

${ }^{84}$ Grundlegend wohl G.F. Schuppert Rückzug des Staates?, DÖV 1995, 761 (768); ders. Vom produzierenden zum gewährleistenden Staat: Privatisierung als Veränderung staatlicher Handlungsformen, in: König/Benz (Hrsg.) Privatisierung und staatliche Regulierung, 1997, 539 (550 f.); W. Hoffmann-Riem Verfahrensprivatisierung als Modernisierung, in: Hoffmann-Riem/Schneider (Hrsg.) Verfahrensprivatisierung im Umweltrecht (1996), 9 (24); ders. Tendenzen der Verwaltungsrechtsentwicklung, DÖV 1997, 433 (441 f.). Im Anschluss z.B. Schoch (Fn. 22), 204, 212; M. Ruffert Regulierung im System des Verwaltungsrechts, AöR 124 (1999), 237 (247); J.-P. Schneider Liberalisierung der Stromwirtschaft durch regulative Marktorganisation, 1999, 123-125; Weiß Privatisierung (Fn. 75), 291-347; R. Ruge, Die Gewährleistungsverantwortung des Staates und der Regulatory State, 2004, insbes. 172-188; G. Britz „Kommunale Gewährleistungsverantwortung" - Ein allgemeines Element des Regulierungsrechts in Europa?, Verw. 37 (2004), 145 (148 f.); Kühling Regulierung (Fn. 41), 557-562; ders. (Fn. 42), DVB1 2013, 1093 (1095 und 1101); M. Burgi Privatisierung, in: HStR IV (Fn. 50), § 75 Rn. 3; Brosius-Gersdorf (Fn. 68), VerwArch 98 (2007), 317 (325, 340); W. Kahl Die Privatisierung der Wasserversorgung, GewArch 2007, 441 (442 f.); Franzius Gewährleistung (Fn. 29), 121-134; Brehme Privatisierung (Fn. 72), 51; Schulze-Fielitz (Fn. 82), § 12 Rn. 158-165; Krajewski Grundstrukturen (Fn. 45), 281-290; M. Schiller Staatliche Gewährleistungsverantwortung und die Sicherstellung von Anschluss und Versorgung im Bereich der Energiewirtschaft, 2012. Als „Gewährleistungspflicht für die Erfüllung von Verwaltungsaufgaben“ bei Bauer Privatisierung (Fn. 6), 268; ähnlich Kämmerer Privatisierung (Fn. 69), 474 479; als „Sicherstellungsauftrag“ bei Butzer (Fn. 50), § 74 Rn. 1-3. Früher schon als „Garantenstellung“ des Staates bei Gallwas VVDStRL 29 (1971), 211 (226-232). 
Private in die Aufgabenerfüllung eingeschaltet sind bzw. ihnen die Erbringung von Daseinsvorsorgeleistungen überlassen ist, muss er ihre Tätigkeit überwachen und auf die Erfüllung des Versorgungsauftrags achten. Der Staat kann sich m.a.W. im Rahmen der Verfassung aus der „Erfüllungsverantwortung" zurückziehen, solange er einen rechtlichen Ordnungsrahmen bereitstellt und sich durch rechtliche Instrumente einen hinreichenden Einfluss sichert, der die Gewährleistung der Versorgung verspricht. Verallgemeinernd mündet dieser Ansatz in die Rede vom „Gewährleistungsstaat" ${ }^{\text {"85 }}$, dessen Aufgaben von einer "Gewährleistungsverwaltung“"86 und durch "Gewährleistungskommunen"87 wahrgenommen werden und der mit den Instanzen der EU in einem „Gewährleistungsverbund“ zusammengespannt ist ${ }^{88}$. Diese Terminologie hat sich heute weitgehend

${ }^{85}$ Statt aller M. Eifert Grundversorgung mit Telekommunikationsleistungen im Gewährleistungsstaat, 1998, 18-22; G. F. Schuppert Der moderne Staat als Gewährleistungsstaat, in: Schröter (Hrsg.) Empirische Policy- und Verwaltungsforschung, FS Wollmann, 2001, 399; W. Hoffmann-Riem Modernisierung von Recht und Justiz. Eine Herausforderung des Gewährleistungsstaates, 2001; C. Franzius Der „Gewährleistungsstaat" - Ein neues Leitbild für den sich wandelnden Staat?, Der Staat 42 (2003), 493; ders. Gewährleistung (Fn. 29), 77-121; ders. Der Gewährleistungsstaat, VerwArch 99 (2008), 351; Knauff Gewährleistungsstaat (Fn. 45), insbes. 59-91; ders. Gewährleistungsstaatlichkeit in Krisenzeiten: Der Gewährleistungsstaat in der Krise, DÖV 2009, 581; div. Beiträge in Schuppert (Hrsg.) Der Gewährleistungsstaat - Ein Leitbild auf dem Prüfstand, 2005; K. Waechter Verwaltungsrecht im Gewährleistungsstaat, 2008; Isensee (Fn. 66), § 73 Rn. 72; M. Ruffert Begriff, in: Fehling/Ruffert (Fn. 20), § 7 Rn. 31; Hellermann Schutz (Fn. 8), 389 f.; D. Krausnick Staat und Hochschule im Gewährleistungsstaat, 2010; Krajewski Grundstrukturen (Fn. 45), 549-563; H. Wißmann in: Hoffmann-Riem/Schmidt-Aßmann/Voßkuhle (Hrsg.) GVwR, Bd. I, 2. Aufl. 2012, § 15 Rn. 14 und 63.

${ }^{86}$ Statt vieler Kämmerer Privatisierung (Fn. 69), 478; H. Lackner Gewährleistungsverwaltung und Verkehrsverwaltung, 2004; E. Schmidt-Aßmann Das Allgemeine Verwaltungsrecht als Ordnungsidee, 2. Aufl. 2004, 3. Kap, Rn. 116; F. Schoch Gewährleistungsverwaltung - Stärkung der Privatrechtsgesellschaft?, NVwZ 2008, 241; Franzius Gewährleistung (Fn. 29), 134-145: Schulze-Fielitz (Fn. 82), § 12 Rn. 51-63; M. Pechstein Umbau der Wasser- und Schiffahrtsverwaltung von einer Ausführungsverwaltung zu einer Gewährleistungsverwaltung?, DÖV 2013, 85. Für ein „Gewährleistungsverwaltungsrecht" grundlegend Voßkuhle Beteiligung Privater (Fn. 7), 304-306; daran anschließend z.B. Wollenschläger Rückholoptionen (Fn. 77), 25-34; M. Burgi Rechtsregime, in: GVwR, Bd. I, 2. Aufl. 2012, § 19 Rn. 79 f.

87 Vgl. J. Libbe/J.H. Trapp/St. Tomerius Gemeinwohlsicherung als Herausforderung umweltpolitisches Handeln in der Gewährleistungskommune, 2004; Knauff Gewährleistungsstaat (Fn. 45), 203.

${ }^{88}$ Franzius Gewährleistung (Fn. 29), 151; Krajewski Grundstrukturen (Fn. 45), 576587, der diesen Verbund allerdings noch unvollendet bzw. in statu nascendi sieht. Für die EG als „Gewährleistungsgemeinschaft“ W. Hoffmann-Riem, Telekommunikationsrecht als europäisiertes Verwaltungsrecht, DVBl 1999, 125; Schoch (Fn. 86), NVwZ 2008, 241 (243). 
durchgesetzt, zumal sie ja in der Verfassung für Eisenbahnen, Post und Telekommunikation konkrete sprachliche Anknüpfungspunkte findet. Für die Infrastruktur als Gegenstand staatlicher Gewährleistung ergibt sich dann die Bezeichnung als „Infrastrukturverantwortung“. ${ }^{89}$

Seinen Infrastrukturauftrag kann der Staat - gleichsam als Gewährleistungsstaat, der sich zur Erfüllungsverantwortung aufschwingt - selbst erfüllen durch öffentliche Einrichtungen oder Unternehmen, wie sie traditionell z.B. in der kommunalen Wasserversorgung ${ }^{90}$ und Abwasserbeseitigung ${ }^{91}$ oder in Gestalt von öffentlichen Schulen, Bibliotheken und Friedhöfen anzutreffen sind. Ebenso begründet die Überlassungspflicht nach $\S 17$ Abs. $1 \mathrm{KrWG}$ zunächst ein Entsorgungsmonopol der durch sie berechtigten öffentlich-rechtlichen Entsorgungsträger. ${ }^{92}$ Bau und Unterhaltung von Straßen und Wasserstraßen obliegt in Deutschland traditionell den öffentlichen Straßenbaulastträgern (stellvertretend § 3 FStrG, § 7 Abs. 1 WaStrG) und ist damit grundsätzlich als staatliche Aufgabe ausgestaltet.

Alternativ kann der Gewährleistungsstaat die Infrastruktur im Rahmen der Verfassung für private Trägerschaft und private Leistungserbringung öffnen. Die Beteiligung Privater an der Erfüllung öffentlicher Auf-

89 Fehling (Fn. 67), AöR 121 (1996), 59 (insbes. 81); Hermes Infrastrukturverantwortung (Fn. 11), 337-340; Schoch (Fn. 22), 198; Eifert Grundversorgung (Fn. 85), 175; Cornils (Fn. 79), 378; V. Mehde Staatliche Infrastrukturverantwortung im Praxistest Lehren aus der Entprivatisierung des britischen Schienennetzbetreibers Railtrack, ZögU 25 (2002), 421; Glöckner Infrastrukturverantwortung (Fn. 11); Remmert in: Epping/Hillgruber (Hrsg.) GG, 2. Aufl. 2013, Art. 89 Rn. 14 und Art. 90 Rn. 14; Kotzur (Fn. 28), JöR n.F. 61 (2013), 195 (201). Zum Begriff s. auch schon die Gegenäußerung der Bundesregierung in BT-Drucks. 12/5015 (1993), 16, zu Nr. 7.

90 Zur Marktstruktur in der Wasserversorgung vgl. z.B. die Angaben bei Brehme Privatisierung (Fn. 72), 64-75, die von $96 \%$ öffentlichen Versorgungsunternehmen ausgeht; ebenso W. Kahl Wasser, in: Fehling/Ruffert (Fn. 20), § 14 Rn. 1; St. Keller Wasserversorgung, in: Krautscheid, Daseinsvorsorge (Fn. 60), 179 (180-182). S. auch z.B. J. Hellermann Wasserversorgung als Gegenstand kommunaler Daseinsvorsorge, in: Ipsen (Hrsg.) Wasserversorgung zwischen kommunaler Daseinsvorsorge und marktwirtschaftlichem Wettbewerb, 2003, 38; W. Frenz Liberalisierung und Privatisierung der Wasserwirtschaft, ZHR 166 (2002), 307.

$91 \S 56$ WHG schreibt die Abwasserbeseitigungspflicht für juristische Personen des öffentlichen Rechts fest, die sich hierzu allerdings Dritter bedienen können. Zu den sich daraus ergebenden Optionen z.B. H. Bauer Privatisierungsimpulse und Privatisierungspraxis in der Abwasserentsorgung, VerwArch 90 (1999), 561; D. Zacharias Privatisierung der Abwasserbeseitigung, DÖV 2001, 454.

92 Vgl. W. Kahl Abfall, in: Fehling/Ruffert (Fn. 20), § 13 Rn. 7 f. Zur Beauftragung Dritter vgl. § 22 KrWG; zum Ganzen $M$. Gädeke Öffentliche und private Entsorgungsverantwortung, 2002. 
gaben stellt nicht nur, wie Voßkuhle zu Beginn seines Staatsrechtslehrerreferats hervorgehoben hat ${ }^{93}$, in freiheitlichen Ordnungen eine Alltäglichkeit dar, sie ist in Bezug auf Infrastruktur und Infrastrukturdienste auch keine Errungenschaft des späten 20. Jhdts. Bereits die erste Welle des Infrastrukturausbaus in Deutschland in den Jahren nach 1850 war gekennzeichnet durch das Engagement von Privatunternehmen, die mit dem Staat durch spezifische Verträge verbunden waren, welche ihnen das Monopol z.B. für die Gas- oder Wasserversorgung bestimmter Städte oder Stadtteile zuwiesen. ${ }^{94}$ Und auch das Preußische Eisenbahngesetz von 1838 hatte bereits zur Konzessionierung von konkurrierenden Bahnen auf fremden Strecken ermächtigt. ${ }^{95}$

Für diese private Bereitstellung von Infrastruktur und Infrastrukturdiensten stehen grundsätzlich drei Wettbewerbsmodelle zur Verfügung, die in ihrer praktischen Anwendung zum Teil auch kombiniert werden ${ }^{96}$ : Wettbewerb in der Infrastruktur, um die Infrastruktur oder zwischen Infrastrukturen.

- Es kann Wettbewerb ,in der Infrastruktur“ geben, wenn mehrere Leistungsanbieter gleichzeitig eine einzige Infrastruktur nutzen, um ihre Leistungen zu erbringen. Ausdruck dieses Modells ist regelmäßig die gesetzliche Eröffnung diskriminierungsfreien Zugangs von Anbietern zur Infrastruktur, wie ihn das Gesetz z.B. für Eisenbahnleistungen ( 14 $\mathrm{AEG}^{97}$ ), die Festnetztelefonie ( $\$ \S 21-23 \mathrm{TKG}$ ), die Strom- und Gasversorgung ( $\$ 20, \S \S 26-28$ EnWG) oder die Bodenabfertigungsdienste auf

93 Voßkuhle Beteiligung Privater (Fn. 7), 268.

${ }^{4}$ Dazu L. Jellinghaus Zwischen Daseinsvorsorge und Infrastruktur. Zum Funktionswandel von Verwaltungswissenschaften und Verwaltungsrecht in der zweiten Hälfte des 19. Jahrhunderts, 2006, 214-220; s. auch Schneider Liberalisierung (Fn. 84), 60-62; Ruge (Fn. 84), 190-192; Brehme Privatisierung (Fn. 72), 111-114. Zur Frühgeschichte des ÖPNV Knauff Gewährleistungsstaat (Fn. 45), 321-334.

${ }_{95}$ Vgl. Fehling (Fn. 67), AöR 121 (1996), 59 (68); F. Schorkopf Regulierung nach den Grundsätzen des Rechtsstaates, JZ 2008, 20 (27), der hierin ein frühes Regulierungsgesetz sieht. Zum rechtshistorischen Kontext $R$. Michalczyk Europäische Ursprünge der Regulierung im Wettbewerb, 2010, 125-175; P. Cancik Verwaltung und Öffentlichkeit in Preußen, 2007, 348-354.

${ }_{96}$ Zum folgenden z.B. Chr. Theobald Aktuelle Entwicklungen des Infrastrukturrechts, NJW 2003, 324 (325-328).

${ }^{97}$ In Verbindung mit der Verordnung über den diskriminierungsfreien Zugang zur Eisenbahninfrastruktur und über die Grundsätze zur Erhebung von Entgelt für die Benutzung der Eisenbahninfrastruktur (EIBV) v. 3.6.2005 (BGBl. I 1566). Zum unionsrechtlichen Hintergrund vgl. Art. 10-13 RL 2012/34/EU zur Schaffung eines einheitlichen europäischen Eisenbahnraums (ABIEU 2012 L 343/32). 
Verkehrsflughäfen ( $§ 19 \mathrm{c}$ LuftVG $^{98}$ ) gewährleistet. ${ }^{99}$ Für einige Verkehrsmedien ist der Zugang zur Infrastruktur heute abschließend unionsrechtlich geregelt, wie z.B. die Verteilung von Start- und Landezeiten (slots) im Rahmen der Flughafenkoordinierung (§ 27a LuftVG) ${ }^{100}$, für andere steht eine unionsrechtliche Regelung noch bevor. ${ }^{101}$

- Wettbewerb „um die Infrastruktur“ gibt es, wenn eine Infrastruktureinrichtung im Ergebnis nur einem Unternehmen zur Verfügung steht, um seine Leistungen anzubieten. Dies ist z.B. der Fall bei der Vergabe gemeinwirtschaftlicher Linienverkehrsdienste im ÖPNV, die gem. § 8a PBefG und der EU-VO 1370/2007102 grundsätzlich in einem wettbewerblichen Verfahren zu erfolgen hat. Für die Nutzung öffentlicher Verkehrswege zum Betrieb von Energieversorgungsleitungen gewährleistet § 46 EnWG den diskriminierungsfreien Abschluss von Wegenutzungsverträgen mit den Gemeinden. Die kommunale Wasserversorgung und Abwasserbeseitigung werden häufig in Gestalt von Betreiberoder Konzessionsmodellen für geschlossene Versorgungsgebiete an private Versorger vergeben, die für die Laufzeit des entsprechenden Ver-

98 In Verbindung mit der Verordnung über Bodenabfertigungsdienste auf Flugplätzen (BADV) v. 10.12.1997 (BGBl. I. 2885). Zu den dabei zu treffenden Auswahlentscheidungen und ihre gerichtliche Überprüfung z.B. BVerwG NVwZ 2013, 507.

$99 \mathrm{Zu}$ den Zugangsrechten als Kernstück der Infrastrukturregulierung z.B. Fehling Der finanzielle Ausgleich für die Mitbenutzung fremder Infrastruktur bei Schienenwegen, Energieversorgungs- und Telekommunikationsleitungen, VerwArch 86 (1995), 600; ders. (Fn. 67), AöR 121 (1996), 59.

100 Die Flughafenkoordinierung, welche die transparente und diskriminierungsfreie Zuweisung von Zeitnischen zum Starten und Landen auf koordinierten Flughäfen einschließt, erfolgt gem. § 27a Abs. 1 LuftVG nach Maßgabe des europäischen Unionsrechts. Sedes materiae ist insoweit die VO (EWG) Nr. 95/93, zuständig für die deutschen Verkehrsflughäfen (benannt in $\S 1$ der entsprechenden Durchführungsverordnung, FHKV) ist der Flughafenkoordinator der Bundesrepublik Deutschland, eine nachgeordnete Behörde des Bundesministeriums für Verkehr, Bau und Stadtentwicklung in Frankfurt/Main. Als Behörde in diesem Sinne ist durch die Verordnung zur Beauftragung des Flughafenkoordinators (BGBl. 1992 I 2072, geänd. BGBl. 2011 I 1526) eine natürliche Person berufen.

101 Wie z.B. für die Erbringung von Hafendiensten in den Seehäfen der EU, vgl. den Kommissionsentwurf v. 23.5.2013 für eine Verordnung des EP und des Rates zur Schaffung eines Rahmens für den Zugang zum Markt für Hafendienste und für die finanzielle Transparenz der Häfen, KOM(2013) 296 endg.

$102 \mathrm{Zu}$ deren Regime statt aller B. Linke Die Gewährleistung des Daseinsvorsorgeauftrags im öffentlichen Personennahverkehr, 2010, 147-288; A. Heiß Die neue EG-Verordnung für den öffentlichen Personenverkehr - ein Überblick unter der Berücksichtigung der Situation in Deutschland, VerwArch 1000 (2009), 113; M. Nettesheim Das neue Dienstleistungsrecht des ÖPNV - die Verordnung (EG) Nr. 1370/2007, NVwZ 2009, 1449. 
trages eine abgesicherte Monopolstellung erhalten. ${ }^{103}$ Die Sicherung von Wettbewerb und Allgemeinwohl muss hier vorrangig durch ein faires und rechtsstaatlich unterfangenes Vergabeverfahren erfolgen, es handelt sich um ein infrastrukturbezogenes Hauptanwendungsfeld des Vergaberechts.

- Schließlich können Anbieter in einen Wettbewerb „zwischen Infrastrukturen" treten, wenn sie für dasselbe Versorgungsgebiet jeweils eine Infrastruktureinrichtung desselben Typs vorhalten, die substituierbare Leistungen anbieten. Das Standardbeispiel bildet der Mobilfunk, wo ersichtlich ein Wettbewerb der Netze besteht, der durch die gesetzlichen Vorschriften über Frequenzzuteilung gesteuert wird (\$\$ 55-65 TKG) ${ }^{104}$. Seit dem 1.1.2008 mag man als Beispiel auch die Postdienste nehmen, da hier nach dem Auslaufen der „Exklusivlizenz“ der Deutschen Post AG (§ 51 PostG) verschiedene Anbieter mit ihren Sammelund Verteilsystemen miteinander konkurrieren. ${ }^{105}$ Seit der Änderung des PBefG zum 1.1.2013 ${ }^{106}$ stehen zudem die Straßen- und die Schienenbeförderung im Personenfernverkehr im echten Wettbewerb miteinander, da nun $\S 13$ Abs. 2 S. 2 PBefG die Streckengenehmigung nicht mehr an eine Bedürfnisprüfung knüpft.

Eine in diesem Sinne wettbewerblich organisierte Daseinsvorsorge, deren sozialstaatlicher Grundimpuls durch den Einfluss des EU-Binnenmarktrechts und dessen Liberalisierungsimpuls überlagert wird, wirft recht bald die Frage nach den Grenzen von Wettbewerblichkeit im sozialen Rechtsstaat auf. ${ }^{107}$ Unabdingbarer Bestandteil von Infrastrukturverantwortung im Mehrebenensystem ist daher die Aufgabe, beide Impulse in einen sozialverträglichen Ausgleich miteinander zu bringen - eine Auf-

${ }^{103} \mathrm{Zu}$ diesen Modellen z.B. Wollenschläger Rückholoptionen (Fn. 77), 48-58; Brehme Privatisierung (Fn. 72), 198-212; Kahl (Fn. 90), § 14 Rn. 9-11.

${ }_{104} \mathrm{Zu}$ verfassungs- und verwaltungsrechtlichen Fragen der Frequenzordnung z.B. Ruffert (Fn. 84), AöR 124 (1999), 237 (252-265).

${ }_{105}$ Zwar müssen Unternehmen, die gewerbsmäßig Briefsendungen befördern wollen, hierfür eine Lizenz beantragen und in dem Antrag auch das geplante Tätigkeitsgebiet bezeichnen ( $\$ 6$ Abs. 1 PostG), doch ergibt sich hieraus nicht, dass die Lizenzerteilung durch die Bundenetzagentur geschlossene Versorgungsgebiete zuweist und dadurch einen Wettbewerb „zwischen Infrastrukturen“ praktisch verhindert. Für einen „Wettbewerb der Netze" im Bereich der Post schon J. Masing Grundstrukturen eines Regulierungsverwaltungsrechts, Verw. 36 (2003), 1 (17).

106 Vgl. das Gesetz zur Änderung personenbeförderungsrechtlicher Vorschriften v. 14.12.2012 (BGBl. I 2598).

107 Dazu allg. Franzius Gewährleistung (Fn. 29), 56-70 und die Beiträge von M. Potacs und $J$. Kersten Herstellung von Wettbewerb als Verwaltungsaufgabe, in VVDStRL 69 (2010), 254 bzw. 288. 
gabe, deren Erfüllung heute regelmäßig in staatlicher Regulierung und entsprechendem Regulierungsrecht zum Ausdruck kommt. ${ }^{108}$ Dabei kann die staatliche Letztverantwortung für die Infrastrukturversorgung nicht in Frage stehen, sie resultiert vor allem aus der besonderen Legitimation ${ }^{109}$ und der verfassungsrechtlichen Gebundenheit des demokratischen Rechtsstaates. Diese Letztverantwortung kann der europäisch integrierte Verfassungsstaat nur bedingt in den überstaatlichen Verbund einbringen, die Verfassungsgrenzen, welche der europäischen Integration gesetzt sind, gelten auch für die Infrastrukturverantwortung.

\section{Verfassungsrechtliche Ausgestaltungsdeterminanten}

Unter dem Grundgesetz ist der Gestaltungsspielraum des Staates für die Organisation der Infrastrukturversorgung - jenseits von Grundrechten und Sozialstaatsprinzip - durch verfassungsrechtliche Determinanten eingeschränkt. Jenseits des Funktionsvorbehalts in Art. 33 Abs. 4 GG, der für den Wahrnehmungsmodus staatlichen Handelns nur eine geringe Steuerungswirkung entfaltet ${ }^{110}$, ergeben sich spezifische Vorgaben für die Infrastrukturverwaltung vor allem aus den Art. 87d bis 90 GG. Art. 87d Abs. 1 schreibt die Luftverkehrsverwaltung (Luftaufsicht, Flugplankoordinierung, Flugsicherung) als staatliche Aufgabe des Bundes fest, die allerdings nicht mehr in bundeseigener, sondern nur noch ,in Bundesverwaltung" erfüllt werden muss. ${ }^{111}$ Die Flugsicherung, die materiell-recht-

108 Zum - immer noch schillernden - Regulierungsbegriff in diesem Sinne z.B. Ruffert (Fn. 84), AöR 124 (1999), 237 (241-244); ders. Begriff, in: Fehling/Ruffert (Fn. 20), § 7 Rn. 58; Schneider Liberalisierung (Fn. 84), 37; Masing (Fn. 105), Verw. 36 (2003), 1 (29); ders. Soll das Recht der Regulierungsverwaltung übergreifend geregelt werden?, Gutachten D für den 66. Deutschen Juristentag, in: Verhandlungen des 66. DJT (2006), D 5 (D 14); Britz (Fn. 84), Verw. 37 (2004), 145; Kersten (Fn. 107), 316-319.

109 So allgemein schon Schuppert (Fn. 84), DÖV 1995, 761 (769).

110 Denn er gilt nur für die ,Ausübung hoheitsrechtlicher Befugnisse“, also die Ausübung imperativer Staatsgewalt, schreibt aber keine Aufgaben als immerwährend staatliche und schon gar nicht den hoheitlichen Wahrnehmungsmodus fest. Die Verfassungsnorm enthält damit kein Privatisierungsverbot, vgl. U. Di Fabio Privatisierung und Staatsvorbehalt, JZ 1999, 585 (591 f.); Voßkuhle Beteiligung Privater (Fn. 7), 293 mwN; Hünnekens (Fn. 11), 241-243; Masing in: Dreier, GG, Art. 33 Rn. 62. Die hoheitliche Aufgabenerfüllung, wie sie im Zusammenhang mit Infrastruktur z.B. in Art. 87e Abs. 1 S. 1 und Art. 87f Abs. 2 S. 2 GG ausdrücklich festgeschrieben ist, liegt dem Funktionsvorbehalt also voraus und bringt ihn zur Anwendung.

111 Zur Erfassung privatrechtlicher Organisationsformen durch die neue Fassung der Norm z.B. Remmert in: Epping/Hillgruber (Fn. 89), Art. 87d Rn. 6; Schwarz in: Maunz/Dürig, GG, Art. 87d Rn. 10-12 (2011); Windthorst in: Sachs, GG, 6. Aufl. 2011, Art. 87 d Rn. 12. 
lich ohnehin durch vorrangiges Unionsrecht determiniert ist ${ }^{112}$, kann auf unionsrechtlich zertifizierte Organisationen übertragen werden. Für Fernverkehrsleistungen auf der Schiene muss der Bund gem. Art. 87e Abs. 4 GG eine Grundversorgung sicherstellen; hingegen ist in bezug auf die eigentliche Eisenbahninfrastruktur die Eigentümerstellung des Bundes verfassungsrechtlich festgeschrieben (sog. Schienenwegevorbehalt in Art. 87e Abs. 3 S. 2 und 3 GG), lediglich für regional beschränkte Teilmärkte lässt Art. 87e Abs. 5 S. 2 GG eine „Übertragung von Schienenwegen an Dritte" aufgrund Gesetzes zu. Für Post- und Telekommunikationsdienste enthält Art. 87f Abs. 2 S. 1 GG ein Gebot der materiellen Privatisierung ${ }^{113}$, kombiniert mit einem Gewährleistungsauftrag des Bundes und einem Reservat hoheitlicher bundeseigener Verwaltung (Art. 87f Abs. 2 S. 2 GG).

Für die Bundeswasserstraßen schreibt Art. 89 GG die bundeseigene Verwaltung vor. Es handelt sich um eine obligatorische Aufgabenzuweisung an den Bund, die für den Betrieb der Infrastruktur als eine prinzipielle Privatisierungssperre wirkt. ${ }^{114}$ Für die öffentlichen Straßen des Bundes schreibt Art. 90 GG die staatliche Verwaltung durch Bund oder Länder fest. Die staatliche Erfüllungsverantwortung für den Infrastrukturbetrieb ist damit auch hier grundsätzlich von Verfassungs wegen vorgegeben. ${ }^{115}$

112 Vgl. VO 550/2004 über die Erbringung von Flugsicherungsdiensten im einheitlichen europäischen Luftraum (ABIEU L 96/10).

113 Was eine hoheitlich organisierte Leistungserbringung grundsätzlich ausschließt, vgl. BVerfGE 108, 169 (183); Kühling Regulierung (Fn. 41), 570; Windthorst in: Sachs, GG, Art. 87f Rn. 22; Möstl in: Maunz/Dürig, GG, Art. 87f Rn. 36-40 (2010). Dadurch ergibt sich eine außergewöhnlich weitgehende Garantenstellung des Staates, denn er unterliegt einer vorbehaltslosen Erfolgshaftung (,Gewährleistung flächendeckend angemessener und ausreichender Dienstleistungen“), darf aber wegen des Verbots der Eigenerfüllung den Erfolg notfalls nicht selbst herbeiführen, vgl. Cornils (Fn. 79), AöR 131 (2006), 378 (382).

114 Die Erfüllungsverantwortung muss insoweit beim Bund oder den betroffenen Ländern bleiben, vgl. M. Pechstein Umbau der Wasser- und Schiffahrtsverwaltung von einer Ausführungsverwaltung zu einer Gewährleistungsverwaltung?, DÖV 2013, 85 (86 f.) mwN; Gröpl in: Maunz/Dürig, GG, Art. 89 Rn. 90 (2007); Hermes in: Dreier, GG, Art. 89 Rn. 21. Für die Möglichkeit einer Wahrnehmung in Gewährleistungsverantwortung hingegen z.B. B. Remmert in: Epping/Hillgruber (Fn. 89), Art. 89 Rn. 15. Nach $\S \S 7$ Abs. 2 und 12 Abs. 5 WaStrG sollen die hoheitlichen Aufgaben der Unterhaltung, des Aus- oder Neubaus „Dritten zur Ausführung übertragen“ werden können.

115 Kühling Regulierung (Fn. 41), 544 f.; Gröpl in: Maunz/Dürig, GG, Art. 90 Rn. 77 (2007). Zu den Möglichkeiten einer funktionalen Privatisierung z.B. Gröpl ebd., Rn. 7986a; Uerpmann-Wittzack (Fn. 52), Rn. 35-37. Nach dem BFStrPrivFinG 1994 (neugef. in BGBl. 2006 I 49) kann Privaten der Bau, die Erhaltung, der Betrieb und die Finanzierung von Bundesfernstraßen zur Ausführung übertragen werden. 
Unter dem Strich enthält die Verfassung nur für einzelne Infrastrukturen überhaupt Vorgaben, und diese sind schon im grundsätzlichen Zugriff sehr heterogen. Immerhin ergibt sich, dass das GG für die geregelten Infrastrukturen ersichtlich von einer fortbestehenden Verantwortung des Staates ausgeht und eine völlige Freigabe für privatwirtschaftlichen Wettbewerb nicht zulässt: Der soziale Rechtsstaat muss die fraglichen Infrastrukturleistungen gewährleisten und darf den Betrieb bzw. die Verwaltung der Infrastruktur selbst nur in sehr engen Grenzen aus den Händen geben.

\section{Fragen des Verantwortungsinhalts}

Der Inhalt der staatlichen Infrastrukturverantwortung wird sowohl durch ihre rechts- und sozialstaatliche Grundierung als auch durch ihre unionsrechtliche Überlagerung bestimmt. Der verfassungsstaatliche Impuls erzwingt vor allem die Sicherung der Funktionsfähigkeit, Vorkehrungen für eine gleichmäßige, sozialverträgliche Versorgung, die Sicherung wesentlicher öffentlicher Interessen (z.B. Umweltschutz, Raumordnung, Städtebau) sowie den Ausgleich mit Rechten und Interessen Dritter in bezug auf die Nutzung der Infrastruktur. Die unionsrechtliche Überlagerung streitet - vor allem bei den durch Unionssekundärrecht liberalisierten Infrastrukturleistungen (Luftverkehr, Telekommunikation, Post, Energieversorgung, Eisenbahn, ÖPNV) - für die Ermöglichung privaten Wettbewerbs, so dass Wettbewerbsschutz und die subjektiven Interessen der Wettbewerber Teil der (europäisch determinierten) Infrastrukturverantwortung des Staates sind ${ }^{116}$. Äußerlicher Ausweis dieser gleichsam „doppelten" Verantwortung ist, dass in den regulierten Wirtschaftsbereichen die Gesetzeszwecke Versorgungssicherheit und Wettbewerbsförderung durch Netzregulierung ausdrücklich nebeneinander stehen (z.B. in $\S 1$ TKG, § 1 I, II EnWG, § 1 I AEG, § 1 PostG).

Soweit der Staat - unter dem Druck des Unionsrechts oder ohne diesen - den Infrastrukturbetrieb oder Infrastrukturleistungen Privaten überlässt, ist er aus Gründen der demokratischen Legitimation und zur Sicherstellung seines verfassungsrechtlichen Infrastrukturauftrags verpflichtet, einen hinreichenden Einfluss auf die Leistungserbringung

116 Ebenso für das Regulierungsverwaltungsrecht Masing DJT-Gutachten (Fn. 108), D 14, D 60 f. Der Mißbrauchstatbestand in § 19 Abs. 2 Nr. 4 GWB ist übergreifender Ausdruck dieses erweiterten Schutzauftrags und verankert ihn im einfachen Gesetz. 
sicherzustellen. ${ }^{117}$ Daraus ergeben sich für ein wettbewerbsorientiertes „Infrastrukturrecht“ bestimmte Grundanforderungen:118

(1) Der Staat muss die Leistungsstandards der Grundversorgung im Rahmen der verfassungs- und unionsrechtlichen Determinierung verbindlich festlegen. Für die Bereiche Post und Telekommunikation findet sich diese Festlegung in der Verfassung selbst, wenn Art. 87f Abs. 1 GG zur Gewährleistung ,flächendeckend angemessener und ausreichender Dienstleistungen" verpflichtet. Dieser abstrakte Standard wird durch das gesetzlich fixierte Konzept der Universaldienste konkretisiert. ${ }^{119}$ Für die Energieversorgung bestehen Grundpflichten der Netzbetreiber (§§ 11-16a EnWG) und eine Grundversorgungspflicht (§ 36 EnWG $\left.{ }^{120}\right)$. Für den ÖPNV ergeben sich die Anforderungen an Umfang und Qualität des Verkehrsangebotes aus den Nahverkehrsplänen gem. § 8 III PBefG. Im Eisenbahnrecht wirken als Funktionsäquivalente der Personen- und Gepäckbeförderungsanspruch (§ 10 AEG) sowie die Genehmigungspflichtigkeit der Streckenstillegung (§ 11 AEG). ${ }^{121}$ Im Rahmen vertraglicher Kooperationsverhältnisse ist der Versorgungsstandard in entsprechenden Leistungsvereinbarungen festzuschreiben. ${ }^{122}$

Inhaltlich kommt dem Staat hier ein erheblicher politischer Gestaltungsspielraum zu, der rechtlich nur durch das sozialstaatliche Minimum sowie durch seine verfassungsrechtliche Integrationsaufgabe begrenzt ist. Letztere ist dabei vor allem für die Infrastrukturversorgung in der Fläche auch an dem sozialen Faktum einer ,schrumpfenden Gesellschaft" ${ }^{\text {"123 }}$ aus-

117 Vgl. Voßkuhle Beteiligung Privater (Fn. 7), 296: verfassungsrechtliche Pflicht, den Mangel an tatsächlicher Entscheidungsbeherrschung durch entsprechende Sicherungsvorkehrungen adäquat auszugleichen. Zum kommunalen Handeln in Kooperationsverhältnissen entsprechend Brehme Privatisierung (Fn. 72), 307-315.

118 Vgl. die „Grundbausteine des Gewährleistungsverwaltungsrechts“ bei Voßkuhle Beteiligung Privater (Fn. 7), 310 f.; daran anschließend z.B. Wollenschläger Rückholoptionen (Fn. 77), 27-32; sehr ähnlich Butzer (Fn. 50), § 74 Rn. 51-54.

119 Vgl. $\S \S 78,79$ TKG, § 11 PostG iVm Post-UniversaldienstleistungsVO (PUDLV). Grundlegend zum Konzept und seiner verfassungsrechtlichen Verankerung z.B. K. Windthorst Der Universaldienst im Bereich der Telekommunikation, 2000, 112-387.

120 Konkretisiert in der Verordnung über Allgemeine Bedingungen für die Grundversorgung von Haushaltskunden und die Ersatzversorgung mit Elektrizität aus dem Niederspannungsnetz (StromGVV) und die Verordnung über Allgemeine Bedingungen für die Grundversorgung von Haushaltskunden und die Ersatzversorgung mit Gas aus dem Niederdrucknetz (GasGVV), beide v. 26.10.2006.

121 Kühling Regulierung (Fn. 41), 156.

122 Vorgesehen z.B. für die Kinder- und Jugendhilfe in $\S 78 \mathrm{c}$ Abs. 1 SGB VIII, für die Rehabilitation und Teilhabe Behinderter in $\S 21$ Abs. 1 SGB IX; für die integrierte Arztversorgung in $\S 140$ b Abs. 2 SGB V.

123 F. X. Kaufmann Schrumpfende Gesellschaft. Vom Bevölkerungsrückgang und seinen Folgen, 2005. 
zurichten: Das Leitbild der „gleichwertigen Lebensverhältnisse“, auf das Art. 72 Abs. 2 GG das föderale System noch immer festzulegen scheint und das z.B. in $\S 2$ Abs. 2 Nr. 1 ROG in ,ausgeglichene infrastrukturelle Verhältnisse" übersetzt ist, wird daher im Sinne eines Mindestmaßes an sozialem Zusammenhalt zu verstehen sein, das eine differenzierte Entwicklung der Infrastruktur durchaus zulässt. ${ }^{124}$ Die begriffsimmanente Entwicklungsoffenheit der Daseinsvorsorge bildet das Einfallstor, um Herausforderungen wie die demografische Entwicklung oder die Überschuldung der öffentlichen Haushalte zu verarbeiten.

(2) Der Staat muss die Auswahl des privaten Leistungserbringers gesetzlich in einer Weise regeln, dass sie nach objektiven, sachorientierten Gesichtspunkten erfolgt und dass dabei durch den Versorgungsauftrag bestimmte Qualitäts- und Leistungsanforderungen beachtet werden. Beim Wettbewerb um die Infrastruktur muss regelmäßig ein diskriminierungsfreies und rechtsschutzbewehrtes Vergabeverfahren stattfinden, das sich entweder nach speziellen gesetzlichen Bestimmungen richtet ${ }^{125}$ oder jedenfalls durch Art. 3 Abs. 1 GG determiniert ist ${ }^{126}$. Besteht potentiell eine grenzüberschreitende Relevanz der in Frage stehenden Beauftragung, so ist zudem das Vergabeprimärrecht der EU-Grundfreiheiten zu beachten. ${ }^{127}$ Beim Wettbewerb in der Infrastruktur wird die Auswahlentschei-

124 Kersten (Fn. 68), DVB1 2006, 942 (945-949) bzw. IzR 2008, 1 (3-6); ebenso für die kommunale Daseinsvorsorge Brosius-Gersdorf (Fn. 68), VerwArch 98 (2007), 317 (333339). Vgl. auch $R$. Winkel Öffentliche Infrastrukturversorgung im Planungsparadigmenwandel, IzR 2008, 41. Ganz in diesem Sinne beschreibt das BVerfG in seiner Rechtsprechung zur Gesetzgebungskompetenz des Bundes die „Gleichwertigkeit der Lebensverhältnisse" nur noch als unterste Schwelle des sozialen Zusammenhalts, vgl. BVerfGE 106, 62 (144); 111, 226 (253); 112, 226 (244).

125 Wie z.B. in den $\$ \S 97$ ff. GWB (Kartellvergaberecht), konkretisiert z.B. durch die Verordnung über die Vergabe von Aufträgen im Bereich des Verkehrs, der Trinkwasserversorgung und der Energieversorgung (Sektorenverordnung - SektVO), oder nach 8b PersBefG iVm Art. 5 Abs. 3 VO (EG) Nr. 1370/2007; für Bodenabfertigungsdienste auf Flugplätzen vgl. § 19c LuftVG iVm $§ 7$ BADV. Allgemein für die Wasserversorgung: Brehme Privatisierung (Fn. 72), 430-440.

${ }_{126}$ Zur verfahrenssteuernden Wirkung des allgemeinen Gleichheitssatzes bei staatlichen Verteilungsverfahren z.B. BVerfGE 116, 135 (153 f.); Th. Puhl Der Staat als Wirtschaftssubjekt und Auftraggeber, VVDStRL 60 (2001), 456 (478-480); H. Pünder Zu den Vorgaben des grundgesetzlichen Gleichheitssatzes für die Vergabe öffentlicher Aufträge, VerwArch 95 (2004), 38 (42-53); F. Wollenschläger Verteilungsverfahren, 2010, 203 f.; O. Dörr Vergaberecht in Deutschland: Das Recht der öffentlichen Auftragsvergabe im Stufenbau der Rechtsordnung, in: Dreher/Motzke (Hrsg.) Beck'scher Vergaberechtskommentar, 2. Aufl. 2013, Einleitung, Rn. 75.

${ }_{127} \mathrm{Zu}$ diesem z.B. F. Wollenschläger Das EU-Vergaberegime für Aufträge unterhalb der Schwellenwerte, NVwZ 2007, 388; J. Kühling/F. Huerkamp in: Montag/Säcker (Hrsg.) Münchener Kommentar zum Europäischen und Deutschen Wettbewerbsrecht 
dung ersetzt durch das gesetzlich garantierte Marktzutrittsrecht aller Wettbewerber (diskriminierungsfreier Netzzugang), dem Herzstück der unionsrechtlich vorgeformten Regulierung. ${ }^{128}$ Qualifikationsanforderungen ergeben sich hier mitunter daraus, dass die Leistungserbringung als solche kraft Gesetzes genehmigungspflichtig ist. ${ }^{129}$

$\mathrm{Zu}$ den Leistungsanforderungen, ob gesetzlicher oder vertraglicher Provenienz, muss angesichts des Versorgungscharakters der Infrastruktur stets ihre allgemeine Zugänglichkeit und die diskriminierungsfreie Erbringung der Infrastrukturleistungen für den Endnutzer gehören. Bei Einrichtungen der kommunalen Daseinsvorsorge erfüllt diese Funktion der allgemeine kommunalrechtliche Zugangsanspruch ${ }^{130}$, der ggf. in Gestalt eines Kontrahierungszwangs auf einen privaten Betreiber überzuleiten ist. ${ }^{131}$

(3) Über die Leistungserbringung muss der Staat eine geeignete „Gewährleistungsaufsicht" ${ }^{\text {"132 }}$ ausüben, die sich neben der Erfüllung des festgelegten Leistungsstandards und der allgemeinen Zugänglichkeit sowohl auf den Wettbewerbs- als auch auf den Kunden- und Verbraucherschutz beziehen muss. ${ }^{133}$

(4) Eine Kernaufgabe des Staates in den regulierten Netzwirtschaften ist die Entgeltregulierung ${ }^{134}$. In ihr kommt die dichotome staatliche Ver-

(Kartellrecht), Bd. 3: Beihilfen- und Vergaberecht, 2011, vor $§ 97$ ff. GWB Rn. 6-144; Dörr (Fn. 126), Rn. 142-170.

128 Vgl. § 19 TKG, § 20 EnWG, § 14 AEG; ähnlich § 29 PostG; für den Luftverkehr $\S 15$ Abs. 1 VO (EG) Nr. 1008/2008. Zur Typologie und den Grundstrukturen der Netzzugangsansprüche Kühling Regulierung (Fn. 41), 182-284.

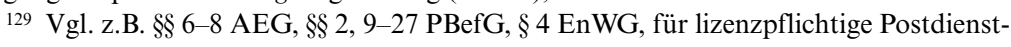
leistungen $\S \S 5-9$ PostG.

${ }^{130} \mathrm{Im}$ vorliegenden Zusammenhang z.B. Brehme Privatisierung (Fn. 72), 161 f. Allgemein statt aller M. Geis Kommunalrecht, 2. Aufl. 2011, § 10 Rn. 11-52; M. Burgi Kommunalrecht, 4. Aufl. 2012, § 16 Rn. 15-19.

131 Vgl. z.B. Brehme Privatisierung (Fn. 72), 215 f.; M. Fehling Instrumente und Verfahren, in: Fehling/Ruffert (Fn. 20), § 20 Rn. 48-52. Diese sowohl wettbewerblich wie sozialstaatlich radizierte Zugangsverantwortung des Staates kommt z.B. auch zum Ausdruck in den Regeln zur Sicherstellung der sog. Netzneutralität im Telekommunikationsrecht (§ 41a TKG): zur aktuellen Debatte um eine geplante VO zur Netzneutralität z.B. Fetzer FAZ v. 21.8.2013, 19; allg. die Beiträge in Spiecker gen. Döhmann/Krämer (Hrsg.) Network Neutrality and Open Acess, 2011; N. Wimmer Netzneutralität - eine Bestandsaufnahme, ZUM 2013, 641.

132 Zum Begriff Voßkuhle Beteiligung Privater (Fn. 7), 321; Wollenschläger Rückholoptionen (Fn. 77), 121-139.

${ }_{133} \mathrm{Zu}$ letzterem im Regulierungsrecht vgl. Hellermann und Durner Schutz (jeweils Fn. 8), 377-380 bzw. 412-423.

${ }_{134}$ Vgl. Masing DJT-Gutachten (Fn. 108), D 65 und D 118; Kühling Regulierung (Fn. 41), 83 und ausf. 284-337; als „Herzstück“ der Regulierung bei Schorkopf (Fn. 95), JZ 2008, 20 (22). Zu Aufgaben und Grenzen der Preisregulierung Masing DJT-Gutachten (Fn. 108), D 117-132. 
antwortung in bezug auf die Nutzung der Infrastruktur prägnant zum Ausdruck: die wettbewerbliche Verantwortung in bezug auf Nutzungsentgelte für konkurrierende Leistungsanbieter (Vorleistungsentgelte als Teil der Netzzugangsregulierung), die sozialstaatliche Verantwortung in bezug auf die Preise für Endkunden und Verbraucher. Beide Bindungen liegen der gesetzlich begründeten Genehmigungspflicht bzw. Regulierungsaufsicht ${ }^{135}$ zugrunde.

(5) Schließlich muß der Gewährleistungsstaat für den Fall mangelhafter Leistungserbringung Vorsorge treffen und Nachsteuerungs- oder Rückholoptionen für ein Wiederaufleben der staatlichen „Erfüllungsverantwortung " bereithalten. ${ }^{136}$ Als gesetzliche Instrumente der Erfüllungsvorsorge stehen vor allem die Auferlegung von Universaldienstverpflichtung ${ }^{137}$ bzw. gemeinwirtschaftlichen Leistungen ${ }^{138}$ zur Verfügung. Vertragliche Kooperationsverhältnisse mit privaten Leistungserbringern sind entsprechend auszugestalten, so dass Staat oder Kommune ihrer verfassungsrechtlichen „Ergebnisverantwortung“ für eine hinreichende Versorgung mit Infrastruktur und Infrastrukturleistungen gerecht werden können. ${ }^{139}$ Für die Wahrnehmung dieser unabdingbaren Reservefunktion des Gewährleistungsstaates kommen neben der Verstaatlichung der Infrastruktur und Selbsterbringung der Leistungen grundsätzlich auch andere, weniger invasive Instrumente in Betracht, wie z.B. der Erwerb staatlicher Unternehmensanteile oder der Einkauf der Grundversorgung durch Auftragsvergabe. ${ }^{140}$

135 Vgl. für Nutzungsentgelte z.B. § 14 Abs, 4-5 AEG iVm §§ 21-24 EIBV, §§ 21, 21a, 23a EnWG iVm GasNEV, StromNEV und ARegV, § 19b LuftVG, §§ 27-38 TKG; für Endkundenpreise z.B. § 12 AEG, §§ 39, 40 EnWG, § 39 PBefG; §§ 19-27 PostG; § 39 TKG.

136 Vgl. Voßkuhle Beteiligung Privater (Fn. 7), 326; Schulze-Fielitz (Fn. 82), § 12 Rn. 166; Butzer (Fn. 50), § 74 Rn. 44, 54; Brehme Privatisierung (Fn. 72), 52 f.; ausführlich Wollenschläger Rückholoptionen (Fn. 77), 64-98. Als staatliche „Auffangverantwortung" z.B. bei Weiß Privatisierung (Fn. 75), 336-339; Schoch (Fn. 86), NVwZ 2008, 241 (247). Das Privatisierungsgebot für Post und Telekommunikation in Art. 87f Abs. 2 GG ist in diesem Sinne sozialstaatlich zu arrondieren, vgl. Cornils (Fn. 79), AöR 131 (2006), 378 (383 und 405-408); in diesem Sinne auch BVerfGE 108, 370 (393 f.).

137 Vgl. z.B. $\$ \$ 80-87$ TKG, $\$ \$ 13-17$ PostG.

138 Vgl. z.B. § 8a PBefG und § 15 AEG, jeweils iVm VO (EG) Nr. 1370/2007; für den Luftverkehr Art. 16-18 VO (EG) Nr. 1008/2008.

${ }_{139} \mathrm{Zu}$ entsprechenden Gestaltungen z.B. Bauer Privatisierung (Fn. 6), 276; Wollenschläger Rückholoptionen (Fn. 77), 183-190, 200-208; Brehme Privatisierung (Fn. 72), $440-455$.

140 Cornils (Fn. 79), AöR 131 (2006), 378 (411-419). Zum Subsidiaritätsgebot bei gewährleistungsstaatlichen Notmaßnahmen Knauff (Fn. 85), DÖV 2009, 581 (584). 


\section{Der überstaatliche Rahmen}

Integrierte Verfassungsstaaten ${ }^{141}$ erfüllen die ihnen obliegenden Aufgaben im Rahmen einer gestuften Rechtsordnung, die von einer sachbereichsabhängigen Gemengelage von staatlichem und überstaatlichem Recht mit vielfältigen gegenseitigen Einwirkungen geprägt ist. Das gilt erst recht für die Infrastrukturversorgung als einer Querschnittsmaterie, die etliche verschiedene Lebens- und Rechtsbereiche betrifft und daher unweigerlich mit dem Stufenbau des kompetenzgeteilten Mehrebenensystems in Berührung kommt.

Konkret entstehen infrastrukturbezogene Einwirkungen aus dem materiellen Unionsrecht, die sich ihrerseits wieder in verschiedene Bausteine unterteilen, und - in deutlich geringerem Maße - aus völkerrechtlichen Abreden.

\section{Infrastrukturverantwortung und Unionsrecht}

Die Wahrnehmung der Infrastrukturverantwortung durch den europäischen Verfassungsstaat wird durch den materiell-rechtlichen Rahmen des Unionsrechts nicht nur begrenzt, sie erhält aus diesem auch eigene Impulse. $\mathrm{Zu}$ den Anforderungen an ein ,zukunftsfähiges Infrastrukturrecht“ gehört es, diese - vor allem: wettbewerbsorientierten - Impulse aufzunehmen und in den nationalen Ordnungsrahmen zu übertragen, ohne dabei den sozialstaatlichen Versorgungsauftrag aus den Augen zu verlieren. Bei letzterem hilft die Erkenntnis, dass der Versorgungsauftrag, wie gesehen, durch eine europäische Funktionsgarantie verstärkt wird.

Die infrastrukturrelevanten Vorgaben des Unionsrechts bestehen grosso modo aus drei Elementen: der EU-Infrastrukturpolitik, dem Wirtschaftsrecht des Binnenmarktes sowie den besonderen Schutzregeln für Dienstleistungen der Daseinsvorsorge.

\section{a) EU-Infrastrukturpolitik}

Die spezifische Infrastrukturpolitik der Union entfaltet aufgrund ihres begrenzten Anwendungsbereichs und ihrer dünnen Kompetenzausstattung nur eine schwache Determinierungswirkung: Die einschlägigen

141 Zum Begriff $R$. Grawert Der integrierte Verfassungsstaat, in: Lhotta/Oebbecke/ Reh (Hrsg.) Deutsche und europäische Verfassungsgeschichte: sozial- und rechtswissenschaftliche Zugänge, 1997, 133; H. Hofmann Von der Staatssoziologie zu einer Soziologie der Verfassung, JZ 1999, 1065 (1067); ähnlich M. Kaufmann Integrierte Staatlichkeit als Verfassungsprinzip, JZ 1999, 814. 
Art. 170-172 AEUV ermächtigen die Union zwar zur autonomen Förderung grenzüberschreitender Infrastruktur, beziehen sich aber nur auf transeuropäische Netze in den Bereichen Verkehr, Telekommunikation und Energie. Zudem bleibt die Union auf Leitlinien, Aktionen im Bereich der technischen Normung und Unterstützungsmaßnahmen beschränkt ${ }^{142}$ - eine Kompetenz für eine eigenständig gestaltete Infrastrukturpolitik der Union ergibt sich hieraus nicht, vielmehr geht es um die begleitende Steuerung mitgliedstaatlicher Politik. ${ }^{143}$

Immerhin macht aber Art. 170 Abs. 1 AEUV mit seinem ausdrücklichen Verweis auf die Ziele der EU-Kohärenzpolitik deutlich, dass die Basisfunktion der Infrastruktur für die politische, wirtschaftliche und soziale Integration eines Gemeinwesens auch für die Union von verfassungsrechtlicher Bedeutung ist. Außerdem verweist Art. 170 AEUV auf das Binnenmarktziel und unterstreicht damit die Funktion einer Netzinfrastruktur als wesentliche Voraussetzung für grenzüberschreitenden Handel und Freizügigkeit. Für die Union ist Infrastrukturpolitik nicht zuletzt Binnenmarktpolitik.

\section{b) EU-Binnenmarktrecht}

Es verwundert daher nicht, dass die wesentlichen europäischen Impulse für das Infrastrukturrecht aus dem EU-Binnenmarktrecht kommen: Wie in anderen Lebens- und Rechtsbereichen auch (z.B. Sport, Kultur, Bildung, Verteidigung, Steuern, Sozialleistungen), entfaltet sich die innerstaatliche Einwirkungsmacht des Unionsrechts auf das Recht der Infrastrukturen vor allem als eine Funktion der Binnenmarktgestaltung. Das betrifft insbesondere die Netzwirtschaften, die seit den 1990er Jahren auf der Grundlage von Unionssekundärrecht liberalisiert und dadurch zu regulierten Wirtschaftssektoren wurden. ${ }^{144}$

Für die nicht harmonisierten Infrastrukturbereiche ergibt sich ein ähnlicher, wenngleich legislativ ungesteuerter Liberalisierungseffekt aus der unmittelbaren Wirkung der EU-Grundfreiheiten: Da Errichtung und

142 Vgl. z.B. die VO (EU) Nr. 347/2013 zu Leitlinien für die transeuropäische Energieinfrastruktur v. 17.4.2013 (AB1. L 115/39) und dazu z.B. L. Giesberts/A. Tiedge NVwZ 2013, 836; T. Strobel ZEuS 2013, 167; zum Entwurf schon M. Nettesheim Transeuropäische Energieinfrastruktur und EU-Binnenmarkt - Die Neuregelung der TEN-E, in: Giegerich (Hrsg.) Herausforderungen und Perspektiven der EU, 2012, 87.

143 Nettesheim (Fn. 142), 98. Ähnlich P. Schäfer/M. Schröder in: Streinz, EUV/ AEUV, Art. 170 AEUV Rn. 2 und 29.

144 Zusammenfassend z.B. Weiß Privatisierung (Fn. 75), 390-403; Voßkuhle Beteiligung Privater (Fn. 7), 286-288; Kühling Regulierung (Fn. 41), 65-160, für den ÖPNV Knauff Gewährleistungsstaat (Fn. 45), 364-389. 
Betrieb von Infrastruktur Ausweis einer Niederlassung (Art. 49 AEUV), vor allem aber Infrastrukturleistungen regelmäßig Dienstleistungen (Art. 56 AEUV) sind, kommen sowohl der kommerzielle Leistungsanbieter als auch der Endverbraucher als Leistungsempfänger in den Genuss der entsprechenden Diskriminierungs- und Beschränkungsverbote. Hinzu kommt das allgemeine Teilhaberecht für Unionsbürger, das der $\mathrm{EuGH}$ aus Art. 18 iVm Art. 21 AEUV ableitet ${ }^{145}$ und das angesichts seines bislang nicht begrenzten Anwendungsbereichs auch ein Recht auf gleichberechtigten Zugang zur Infrastruktur anderer Mitgliedstaaten umfasst. Mag auch wegen des allfälligen Rechtfertigungspotentials der Gemeinwohlaufgabe Infrastruktur die infrastrukturelle Steuerungswirkung der Grundfreiheiten im praktischen Ergebnis nicht sehr weit tragen ${ }^{146}$, so unterstellt deren grundsätzliche Anwendbarkeit doch Betrieb und Nutzung der nationalen Infrastruktur - einschließlich ihrer rechtlichen Grundlagen - der Aufsicht von Kommission und EuGH. Und die wäre übrigens auch dann herausgefordert, wenn ein EU-Mitgliedstaat die Benutzung seiner öffentlichen Straßen nur für Ausländer entgeltpflichtig machte ...

Die Aufsichtsfunktion der EU-Organe greift erst recht im weiteren Baustein des EU-Binnenmarktrechts, den Regeln über staatliche Beihilfen

145 Vgl. z.B. EuGH Rs. C-184/99, Slg. 2001, I-6193 Rn. 30-37 - Grzelczyk; Rs. C-209/03, Slg. 2005, I-2119 Rn. 31-42 - Bidar; Rs. C-158/07, Slg. 2008, I-8507 Rn. 35-43 - Förster; Rs. C-524/06, Slg. 2008, I-9705 Rn. 69-71 - Huber, Rs. C-103/08, Slg. 2009, I-9117 Rn. 23-28 - Gottwald; Rs. C-73/08, Slg. 2010, I-2735 Rn. 31-33 Bressol u.a.; Rs. C-75/11, Urt. v. 4.10.2012, Rn. 38-51 - Kommission/Österreich; zusammenfassend z.B. Th. Giegerich Unionsbürgerrechte, in: Schulze/Zuleeg/Kadelbach (Hrsg.) Europarecht, 2. Aufl. 2010, § 9 Rn. 56-59.

146 Der Versorgungscharakter der Infrastruktur, ihr notwendiger Raum- und Sozialbezug sowie die Angewiesenheit Dritter auf die damit verbundenen Leistungen begründen regelmäßig ein derart hohes Abwägungspotential zugunsten der öffentlichen Aufgabe, dass sich die staatliche Gestaltungsmacht insoweit häufig durchsetzen wird. Der EuGH erkennt insoweit grundsätzlich hinreichende Allgemeininteressen zur verhältnismäßigen Beschränkung der Binnenmarktrechte einzelner an, vgl. z.B. für den ordnungsgemäßen Betrieb des öffentlichen Fernsprechnetzes z.B. EuGH Rs. C-18/88, Slg. 1991, I-5941 Rn. 31 - RTT/GB-Inno-BM; verb. Rs. C-388/00, C-429/00, Slg. 2002, I-5845 Rn. 44 - Radiosistemi; für die Gewährleistung des postalischen Universaldienstes EuGH verb. Rs. C-282/04, C-283/04, Slg. 2006, I-9141 Rn. 38 - Kommission/Niederlande; für die Sicherstellung der Energieversorgung z.B. EuGH Rs. C-483/99, Slg. 2002, I-4781 Rn. 47 - Kommission/Frankreich; Rs. C-543/08, Slg. 2010, I-11241 Rn. 84 Kommission/Portugal; für eine funktionsfähige öffentliche Gesundheitsversorgung und die Finanzierbarkeit der sozialen Sicherungssysteme z.B. EuGH Rs. C-158/96, Slg. 1998, I-1931 Rn. 41, 50 f. - Kohll; Rs. C-385/99, Slg. 2003, I-4509 Rn. 67, 73 - MüllerFauré und van Riet; Rs. C-372/04, Slg. 2006, I-4325 Rn. 103-105 - Watts; Rs. C-512/08, Slg. 2010, I-8833 Rn. 33-42 - Kommission/Frankreich. 
(Art. 107-109 AEUV), die als „zusätzliche Rationalitätskontrolle“147 auch auf die Finanzierung von Infrastruktur Anwendung finden. Deren Besonderheiten können bereits die Frage nach der Erfüllung des Beihilfetatbestands aufwerfen, wie etwa nach dem „staatlichen“ Ursprung der Begünstigung bei gesetzlichen Umlageregimen im Bereich der Energieversorgung ${ }^{148}$ oder nach der „Selektivität“ der Begünstigung bei allgemeinnützigen Infrastrukturmaßnahmen ${ }^{149}$. Vor allem kann es bereits an einer beihilferelevanten Begünstigung fehlen, wenn die staatliche Finanzierung nur in transparenten und objektiv erforderlichen Ausgleichsleistungen für die effiziente Erbringung öffentlicher Dienstleistungen besteht: Die Voraussetzungen für eine derartige Tatbestandsreduzierung zugunsten der öffentlichen Versorgung hat der EuGH in seinem mittlerweile berühmten Urteil Altmark Trans aufgestellt ${ }^{150}$; sie sind seitdem Gegenstand zahlreicher konkretisierender Mitteilungen der Kommission ${ }^{151}$, an denen sich die Finanzierungspraxis der Mitgliedstaaten orientiert.

147 Kühling Regulierung (Fn. 41), 17.

148 Zur Befreiung stromintensiver Großabnehmer von den Netzentgelten gem. § 19 Abs. 2 S. 2 StromNEV z.B. Koenig, FAZ v. 24.10.2012, 21; offen gelassen von OLG Düsseldorf, Beschl. v. 14.11.2012 (VI-3 Kart 65/12), BeckRS 2012, 23298, unter II.2.1; Beschl. v. 6.3.2013 (VI-3 Kart 65/12), BeckRS 2013, 6830, unter II vor 1. Zuvor zum Regime des damaligen Stromeinspeisungsgesetzes EuGH Rs. C-379/98, Slg. 2001, I-2099 Rn. 57-66 - PreussenElektra.

149 Dazu z.B. A. Bartosch EU-Beihilfenrecht, 2009, Art. 87 Abs. 1 EGV Rn. 108-110. Allgemein zum Selektivitätsmerkmal z.B. Boysen/Neukirchen (Fn. 49), 130-136; Lübbig/Martin-Ehlers Beihilfenrecht der EU, 2. Aufl. 2009, Rn. 232-234.

${ }^{150}$ EuGH Rs. C-280/00, Slg. 2003, I-7747 Rn. 88-93; mittlerweile stRspr., vgl. z.B. EuGH Rs. C-451/03, Slg. 2006, I-2941 Rn. 60 ff. - Servizi Ausiliari; Rs. C-140/09, Slg. 2010, I-5243 Rn. 35 ff. - Fallimento Traghetti del Mediterraneo; Rs. C-399/08 P, Slg. 2010, I-7831, Rn. 41 ff. - Kommission/Deutsche Post; EuG Rs. T-274/01, Slg. 2004, II-3145 Rn. 130 f. - Valmont Nederland/Kommission; Rs. T-289/03, Slg. 2008, II-81 Rn. 160 ff. - BUPA u.a.; Rs. T-354/05, Slg. 2009, II-471 Rn. 124 ff. - TF1/Kommission; Rs. T-231/06 und T-237/06, Slg. 2010, II-5993, Rn. 125-158 - Niederlande und NOS/ Kommission Zu den Folgen statt aller Boysen/Neukirchen (Fn. 49), 145-165; J. A. Kämmerer Strategien zur Daseinsvorsorge - Dienste im allgemeinen Interesse nach der ,Altmark"-Entscheidung des EuGH, NVwZ 2004, 28; O. Dörr Infrastrukturförderung (nur) nach Ausschreibung?, NZBau 2005, 617; Chr. Arhold in: Münchener Kommentar (Fn. 127), Art. 107 AEUV Rn. 222-238.

151 Vgl. z.B. allgemein die Mitteilung über die Anwendung der Beihilfevorschriften der EU auf Ausgleichsleistungen für die Erbringung von Dienstleistungen von allgemeinem wirtschaftlichen Interesse, ABIEU 2012 C 8/4, Tz. 42-77; insgesamt zum „DAWIPaket" der Kommission M. Knauff Die mitgliedstaatliche Finanzierung der Daseinsvorsorge im Dickicht des europäischen Regelungsgeflechts, ZG 28 (2013), 139. Für einzelne Infrastrukturen hat die Kommission zudem spezielle Leitlinien veröffentlicht, in denen der Begünstigungsausschluss „nach Altmark Trans" stets mitbehandelt ist, vgl. z.B. in ABIEU 2005 C 312/1, Tz. 34-37 (Flughäfen); ABIEU 2009 C 257/1, Tz. 23 f. (Rund- 


\section{c) Daseinsvorsorge als Verfassungswert der Union}

Soweit die staatliche Finanzierung von Infrastruktur jedoch den Beihilfetatbestand erfüllt und nicht durch anderweitige Ausnahmen freigestellt ist ${ }^{152}$, kommt das dritte Element des EU-Infrastrukturrechts zum Tragen: die Anerkennung der Daseinsvorsorge als eigenständiger Verfassungswert, die in Art. 14 AEUV verankert und im entsprechenden Protokoll ${ }^{153}$ mit einer wohlfahrtsstaatlichen Grundierung versehen ist. Gemäß Art. 106 Abs. 2 AEUV kann dieses Verfassungsrechtsgut eine Abweichung von den Wettbewerbsregeln des Vertrages rechtfertigen: Auch für die Infrastrukturversorgung in den Mitgliedstaaten wird hier die Marktorientierung des Unionsrechts zugunsten der öffentlichen Gemeinwohlaufgabe relativiert, sofern diese Aufgabe offiziell und transparent übertragen wurde und die Finanzierung nur dazu dient, die Nettokosten der Versorgungsleistung zu decken. ${ }^{154}$

Unter dem Strich ergibt sich also, dass sich auch jenseits spezifischer Liberalisierungsmaßnahmen der Einwirkungsanspruch des europäischen Unionsrechts auf die Infrastrukturversorgung in den Mitgliedstaaten erstreckt und diese somit der Aufsicht von Kommission und Gerichtshof unterstellt. Dabei erkennt das Unionsrecht grundsätzlich den infrastrukturpolitischen Autonomieanspruch der Mitgliedstaaten an und räumt diesen insoweit einen erheblichen Gestaltungsspielraum ein. Die Infrastrukturverantwortung, einschließlich aller in Betracht kommenden Wahrnehmungsmodi, bleibt also grundsätzlich eine mitgliedstaatliche; die materiell-rechtliche Steuerungswirkung des Unionsrechts ist trotz seines

funk); ABlEU 2013 C 25/1, Tz. 18-27 (Breitbandausbau). Für die Beihilfepraxis zur Infrastrukturförderung s. auch die Infrastructure Analytical Grids der Kommission v. 1.8.2012, die spezielle Leitlinien für die Beurteilung der Finanzierung von Flughafen-, Breitband-, Kultur- und Hafeninfrastrukturen sowie für die der Wasserversorgung enthalten.

${ }^{152} \mathrm{Zu}$ den zahlreichen Ausnahme- und Freistellungstatbeständen des EU-Beihilferechts vgl. z.B. die nach verschiedenen Sektoren untergliederte Darstellung versch. Autoren im Münchener Kommentar (Fn. 127), Teil 2 unter F. Einen deutlichen „Infrastrukturanteil" hat auch die kürzlich betriebene Änderung der Ermächtigungsverordnung zur Freistellung horizontaler Beihilfen (VO 994/98), vgl. den Kommissionsvorschlag v. 5.12.2012 in $\operatorname{COM}(2012) 730$ final.

153 Oben bei Fn. 59.

154 Zum Prüfprogramm nach Art. 106 Abs. 2 AEUV vgl. die Kommissionsmitteilung zum Rahmen der EU für staatliche Beihilfen in Form von Ausgleichsleistungen für die Erbringung öffentlicher Dienstleistungen (ABlEU 2012, C 8/15) und den Kommissionsbeschluss v. 20.12.2011 über die Anwendung von Art. 106 Abs. 2 AEUV auf staatliche Beihilfen in Form von Ausgleichsleistungen zugunsten bestimmter Unternehmen, die mit der Erbringung von Dienstleistungen von allgemeinem wirtschaftlichen Interesse betraut sind (ABIEU 2012 L 7/3). 
umfassenden Einwirkungsanspruchs begrenzt. Dies ändert aber nichts daran, dass auch die Infrastrukturversorgung heute europäisch determiniert ist, dass ein „Infrastrukturrecht“ also nur dann ,zukunftsfähig“ ist, wenn es dieser Determinierung Rechnung trägt.

\section{Infrastruktur im Völkerrecht}

Im Vergleich dazu ist die Einwirkungsintensität des Völkerrechts auf die Errichtung und den Betrieb von Infrastruktur recht gering, dennoch möchte ich als Völkerrechtler diesen Punkt wenigstens kurz erwähnen. Infrastruktur ist ortsgebunden, also dem Staatsgebiet verhaftet und unterliegt damit den allgemeinen Regeln über dessen Nutzung. Es greift daher die Begrenzung des souveränen Staates auf sein eigenes Staatsgebiet, und es greifen die Regeln über die ausnahmsweise Zulässigkeit extraterritorialer Staatsgewalt. ${ }^{155}$ Die ,virtuelle Infrastruktur“ Internet ${ }^{156}$ unterfällt diesen Regeln zum einen durch ihre weiterhin vorhandene territoriale Verankerung in Gestalt von physischen Servern sowie - mit naturgemäß ausdehnender Tendenz - über das sog. Auswirkungsprinzip (,,effects doctrine").

Von der staatlichen Gebietshoheit ausgehend kommen Infrastrukturregeln vor allem als Gegenstand völkerrechtlicher Verträge in Betracht. So gibt es denn auch etliche Abkommen über Regime grenzüberschreitender Infrastruktur: Bekannt ist dies etwa für Schiffahrtswege, wie z.B. den Panama-Kanal, Suezkanal, den Rhein, die Donau oder früher den NordOstsee-Kanal; man mag in einem weiteren Sinne auch die Vereinbarung über die Europäischen Schulen ${ }^{157}$ hierzu rechnen. Daneben stehen Abkommen über die Koordinierung der grenzüberschreitenden Nutzung nationaler Infrastrukturen (Telekommunikation, Post-, Eisenbahn-, StraBen- und Luftverkehr). In eine dieser beiden Kategorien gehörten auch mögliche völkerrechtliche Absprachen über die internationale Kontrolle des Internet, die allerdings im Rahmen der International Telecommunication Union vorerst politisch gescheitert sind. ${ }^{158}$ Nur mittelbar infrastruk-

${ }_{155} \mathrm{Zu}$ diesen z.B. R. Wolfrum in: Dahm/Delbrück/Wolfrum, Völkerrecht, Bd. I/1, 2. Aufl. 1989, 320-328; Stein/v. Buttlar Völkerrecht, 13. Aufl. 2012, Rn. 601-627.

156 Aus jüngerer Zeit z. B. S. E. Schulz/J. Tischer Das Internet als kritische Infrastruktur, ZG 28 (2013), 339.

157 Vereinbarung über die Satzung der Europäischen Schulen zwischen den Europäischen Gemeinschaften und ihren Mitgliedstaaten v. 21.6.1994, ABIEU 1994 L 212/3 (BGBl. 1996 II 2559).

158 Aus Anlass der ITU-Staatenkonferenz zur Überarbeitung der International Telecommunication Regulations im Dezember 2012, vgl. den Konferenzbericht in Vereinte Nationen 2013, 130 f. und D.P. Fidler Internet Governance and International Law. The 
turbezogen sind Vertragsregime, die den grenzüberschreitenden Handel mit Infrastrukturleistungen betreffen, wie z.B. die Dienstleistungsregeln der WTO im General Agreement on Trade in Services (GATS) ${ }^{159}$ oder der Energiecharta-Vertrag ${ }^{160}$.

Darüber hinaus zeigen Fälle wie die Entscheidung des Internationalen Gerichtshofs zum Donaukraftwerk von Gabčíkovo-Nagymaros (1997), dass gelegentlich auch staatliche Infrastruktureinrichtungen zur Debatte stehen, wenn um allgemeine Regeln des Völkerrechts gestritten wird. Nur ist die völkerrechtliche Erfassung der staatlichen Infrastruktur in diesen Fällen eher punktuell und ebenso zufällig, wie wenn die Regeln der UNESCO zum Schutz des Weltkulturerbes anlässlich eines Brückenbaus in Dresden relevant werden. ${ }^{161}$ Dass auch das Internet, die Basisinfrastruktur der modernen Kommunikationsgesellschaft, gegenwärtig vor allem aus der Warte der allgemeinen völkerrechtlichen Regeln gesehen wird, belegen die aktuellen Debatten zum sog. Cyberkonflikt ${ }^{162}$ und den cyber security incidents ${ }^{163}$.

Controversy concerning Revision of the International Telecommunication Regulations, ASIL insights vol. 17, issue 6 (7.2.2013); die offiziellen Konferenzdokumente sind erhältlich unter www.itu.int.

${ }_{159} \mathrm{Zu}$ ihnen z.B. F. Weiss Internationaler Dienstleistungshandel, in: Tietje (Hrsg.) Internationales Wirtschaftsrecht, 2009, § 4; M. Michaelis Dienstleistungshandel (GATS), in: Hilf/Oeter (Hrsg.) WTO-Recht, 2. Aufl. 2010, § 20. Speziell zu öffentlichen Dienstleistungen S. Simon Liberalisierung von Dienstleistungen der Daseinsvorsorge im WTO- und EU-Recht, 2009, 58-179 sowie der kurze Aufriss bei Krajewski Grundstrukturen (Fn. 45), 117-123.

160 Vertrag über die Energiecharta v. 17.12.1994 (BGBl. 1997 II 5); dazu allg. J. Gundel Regionales Wirtschaftsvölkerrecht in der Entwicklung: Das Beispiel des Energiecharta-Vertrages, AVR 42 (2004), 157.

161 Zum Fall der Dresdner Elbschlösschenbrücke z.B. BVerfG 2 BvR 695/07, NVwZ 2007, 1176; U. Fastenrath Der Schutz des Weltkulturerbes in Deutschland, DÖV 2006, 1017; A. v. Bogdandy/D. Zacharias Zum Status der Weltkulturerbekonvention im deutschen Rechtsraum - Ein Beitrag zum internationalen Verwaltungsrecht, NVwZ 2007, 527; M. Kilian Die Brücke über die Elbe - völkerrechtliche Wirkungen des WelterbeÜbereinkommens der UNESCO, LKV 2008, 248; H. Krieger Die Herrschaft des Fremden - zur demokratietheoretischen Kritik des Völkerrechts, AöR 133 (2008), 315 (338-341).

${ }^{162}$ Statt vieler M. N. Schmitt Computer Network Attack and the Use of Force in International Law: Thoughts on a Normative Framework, Columbia JTL 37 (1999), 885; M.C. Waxman Cyber-Attacks and the Use of Force: Back to the Future of Article 2 (4), Yale JIL 36 (2011), 421; T. O. Keber/P.N. Roguski Ius ad bellum electronicum?, AVR 49 (2011), 399.

${ }^{163} \mathrm{Zu}$ diesen vgl. z.B. die Beiträge auf der International Berlin Cyber Conference "Securing the Freedom and Stability of Cyberspace: The Role and Relevance of International Law" am 28.6.2013, Tagungsband i.E. 


\section{Schluss}

Infrastruktur ist die Lebensader jedes Gemeinwesens, da die soziale und politische Gemeinschaft von Menschen eines Mindestmaßes an Versorgung, Mobilität und Kommunikation bedarf. Dies gilt erst recht für moderne Gesellschaften, zu deren Lebensbedingungen die allgegenwärtige Entgrenzung gehört und die weite Teile ihres politischen und sozialen Alltags der technikgestützten Kommunikation anvertraut haben. Für die infrastrukturelle Grundausstattung zu sorgen, ist notwendige Aufgabe des sozialen Rechtsstaates, denn seine Legitimität beruht nicht nur auf dem Schutz vor äußerer Bedrohung und der Sicherstellung inneren Friedens, sondern auch auf der Gewährleistung einer gleichmäßigen und im Prinzip flächendeckenden Versorgung mit den Grundbedürfnissen der modernen Existenz. Dementsprechend ist der Zustand der Infrastruktur und Infrastrukturdienste ein wesentliches Indiz für den Zustand eines politischen Gemeinwesens, seiner Volkswirtschaft und seiner Verfassungskultur.

„Infrastrukturrecht" dient der rechtlichen Gründung und Flankierung der infrastrukturellen Versorgung. Es handelt sich nicht um ein kohärentes Teilrechtsgebiet, sondern um eine deskriptive Sammelbezeichnung aus dem Bereich der Daseinsvorsorge. Deren sozialstaatlich radizierten Verfassungsauftrag können Staat und Kommunen auch in Bezug auf den Betrieb und die Nutzung der Infrastruktur in verschiedenen Modi wahrnehmen, solange die Letztverantwortung bei der verfassungsrechtlich gebundenen öffentlichen Gewalt liegt. Diese durch die Verfassung gebotene Residualverantwortung setzt auch der unionsrechtlich indizierten Liberalisierung von Netzbetrieb und Leistungserbringung eine letzte Grenze. Das Unionsprimärrecht selbst weist diese Grenze und damit das „europäische Modell der Infrastrukturversorgung“ als ein sozialstaatlich unterfangenes aus, das den Modus der wettbewerblichen Versorgung nur für den Regelfall vorsieht.

Der allgemeine Ordnungsrahmen von Verfassungs- und Unionsrecht läßt dem Staat weite Spielräume, um das Gestaltungs- und Steuerungspotential der Infrastrukturversorgung zu nutzen. Diese Potentiale werden auch dazu dienen müssen, die wesentlichen Zukunftsfragen der infrastrukturellen Versorgung zu beantworten, wie z.B. die Folgen der demografischen Entwicklung, der Überschuldung öffentlicher Haushalte oder das Bedürfnis nach demokratischer Partizipation. Ihren rechtlichen Spielraum beschränken die Staaten vor allem durch die überstaatliche Verabredung infrastrukturbezogenen Handelns, sei es in Europa oder darüber hinaus. Angesichts der allgegenwärtigen Entgrenzung politischer Gemeinschaft liegt die Prognose nahe, dass die „Zukunftsfähigkeit“ des Rechtsnetzgebundener Infrastrukturen vor allem durch internationale Lösungen 
sicherzustellen ist, während die Regeln über punktförmige Infrastrukturen ihre „Zukunftsfähigkeit“ durchaus auch im örtlichen Rahmen unter Beweis stellen können. 
Leitsätze des 1. Referenten über:

\section{Die Anforderungen an ein zukunftsfähiges Infrastrukturrecht}

\section{Einleitung}

1) „Infrastruktur“ ist ständig und überall. Es handelt sich um einen allgegenwärtigen Begriff der Alltags-, Politik-, Verwaltungs- und Rechtssprache, dessen Inhalt vor allem assoziativ oder empirisch angereichert, nicht normativ konturiert ist.

\section{Begriff und Konzept}

\section{Zur Begriffsgeschichte}

2) Der Begriff ist um 1875 als neulateinischer Neologismus im französischen Sprachraum entstanden und erreichte in den 1960er Jahren die deutsche Wissenschaftssprache. Die ökonomische Theorie bezeichnet hiermit eine der Basisfunktionen einer Volkswirtschaft.

\section{Infrastruktur“ als Rechtsbegriff}

3) Seit 1969 wird „Infrastruktur“ in deutschen Gesetzen als Rechtsbegriff verwendet, seit den 1990er Jahren vor allem in den unter Einwirkung des Europarechts regulierten Wirtschaftssektoren.

4) Begriffsprägend sind vor allem drei Elemente: die unmittelbare Allgemeinnützigkeit, der Versorgungszweck sowie die Ortsgebundenheit. Es geht also um ortsfeste Anlagen und Einrichtungen, die der Versorgung der Bevölkerung mit notwendigen Gütern und Dienstleistungen dienen, im Prinzip jedermann zugänglich sind und deren Betrieb von der öffentlichen Hand verantwortet wird. Neben die netzgebundene tritt die punktförmige Infrastruktur, deren Versorgungsleistung vor Ort wirksam wird.

5) „Infrastrukturrecht“ beschreibt die Regeln über die Planung, Errichtung, den Unterhalt und den Betrieb von Infrastruktur. Es handelt es sich nicht um ein kohärentes Teilrechtsgebiet, sondern um eine deskriptive Sam- 
melbezeichnung für eine Teilmenge des Rechts der Daseinsvorsorge, nämlich diejenigen Regeln, die sich auf die Errichtung, Unterhaltung und Nutzung ortsfester Anlagen mit einem staatlich verantworteten Versorgungszweck beziehen.

\section{Infrastruktur und Daseinsvorsorge}

6) Auch in der modernen, von Verantwortungstypologien geprägten Rechtsdogmatik hat die Daseinsvorsorge als Gegenstand staatlicher ,,Verantwortung "ihren Platz.

7) Infrastruktur ist Gegenstand und Instrument der Daseinsvorsorge, steht zu dieser also in einem modalen Verhältnis. „Infrastrukturrecht“ besitzt dementsprechend im Kern einen instrumentellen Charakter, es ist auf die Erfüllung der durch Verfassung oder Gesetz determinierten Versorgungszwecke bezogen und in der Regel beschränkt.

\section{Europäische Konzepte}

\section{Die Infrastrukturverantwortung des Staates}

\section{Verfassungsrechtliche Grundlagen}

8) Infrastruktur ist eine Grundbedingung für die politische Integration jedes Gemeinwesens sowie - gerade in Zeiten einer schrumpfenden Bevölkerung - für den sozialen Zusammenhalt einer Gesellschaft. Die Sicherstellung der zur Erfüllung dieser Integrationsfunktion notwendigen Infrastruktur zählt zu den immanenten Kernfunktionen des Staates und liegt daher von Verfassungs wegen in seiner Letztverantwortung.

9) In der geschriebenen Verfassung des Grundgesetzes ergibt sich ein Verfassungsauftrag zur Infrastrukturvorsorge aus dem Sozialstaatsprinzip sowie aus den grundrechtlichen Schutzpflichten des Staates. Vor allem letztere begründen die staatliche Aufgabe des infrastrukturellen „, Grundrechtsvoraussetzungsschutzes“. Die Direktionskraft dieser verfassungsrechtlichen Grundlagen ist angesichts weiter Gestaltungsspielräume des Gesetzgebers allerdings begrenzt.

10) Art. 14 AEUV enthält eine Funktionsgarantie für die mitgliedstaatliche Daseinsvorsorge und die dafür notwendige Infrastruktur. Die Norm verstärkt nicht nur den verfassungsstaatlichen Auftrag, sondern formulierterst recht in Verbindung mit dem DAI-Protokoll - den öffentlichen Versorgungsauftrag sehr viel klarer und differenzierter als das Grundgesetz. 


\section{Infrastruktur als Gegenstand von Gewährleistungsverantwortung}

11) Die Gewährleistungsverantwortung des Staates für die Daseinsvorsorge impliziert seine prinzipielle Infrastrukturverantwortung, die er sowohl durch Eigenleistung als auch durch die Regulierung privater Leistungserbringung wahrnehmen kann. Für letzteres stehen als Grundmodelle der Wettbewerb in der Infrastruktur, um die Infrastruktur und zwischen Infrastrukturen zur Verfügung.

12) Staatliche Infrastrukturverantwortung im europäischen Mehrebenensystem bedingt die Aufgabe, den sozialstaatlichen Grundimpuls der Daseinsvorsorge und den Liberalisierungsimpuls des EU-Binnenmarktrechts in einen sozialverträglichen Ausgleich zu bringen. Die Verantwortung für diesen Ausgleich kann der integrierte Verfassungsstaat nur vorbehaltich der allgemeinen Grenzen, die der europäischen Integration von Verfassungs wegen gesetzt sind, in den überstaatlichen Verband verlagern.

\section{Verfassungsrechtliche Ausgestaltungsdeterminanten}

13) Für die Ausgestaltung der Infrastrukturverantwortung enthält die Verfassung in den Art. 87d-90 GG nur für einzelne Infrastrukturen überhaupt Vorgaben, und diese sind schon im grundsätzlichen Zugriff sehr heterogen. Immerhin ergibt sich aus ihnen für die betroffenen Infrastrukturen eine fortbestehende staatliche Verantwortung, die eine völlige Freigabe für privatwirtschaftlichen Wettbewerb nicht zulässt. Vor allem darf der soziale Rechtsstaat den Betrieb und die Verwaltung der Infrastruktur selbst nur in sehr engen Grenzen aus den Händen geben.

\section{Fragen des Verantwortungsinhalts}

14) Solange die Infrastrukturverantwortung auf staatlicher Ebene wahrgenommen wird, muss der Verfassungsstaat sowohl dem Versorgungsauftrag als auch dem - vornehmlich unionsrechtlich radizierten - Wettbewerbsauftrag Rechnung tragen. Dazu und aus Gründen der demokratischen Legitimation muss er einen hinreichenden Einfluss auf die private Erbringung von Infrastrukturleistungen sicherstellen.

15) Dies impliziert jedenfalls staatliche Regeln über die Leistungsstandards der Grundversorgung, über die Auswahl des Leistungserbringers, eine Entgeltregulierung für Netzzugang und Endkundenversorgung sowie geeignete Nachsteuerungs- und Rückholoptionen für ein ggf. notwendiges Aufleben der staatlichen „Erfüllungsverantwortung“.

16) Der dem Staat hierbei zukommende politische Gestaltungsspielraum erlaubt es, das Leitbild der „gleichwertigen Lebensverhältnisse“ (Art. 72 Abs. 2 GG) für die Infrastrukturversorgung in der Fläche durch ein Min- 
destmaß sozialen, wirtschaftlichen und politischen Zusammenhalts zu ersetzen und so der Herausforderung einer ,schrumpfenden Gesellschaft" mit einer differenzierten Infrastrukturentwicklung zu begegnen.

\section{Der überstaatliche Rahmen}

\section{Infrastrukturverantwortung und Unionsrecht}

17) Die Einwirkung des Unionsrechts auf die Infrastrukturversorgung in den Mitgliedstaaten ist auch jenseits spezifischer Liberalisierungsmaßnahmen zwar in der Breite umfassend, aber materiell-rechtlich nur von begrenzter Steuerungskraft: Eine Kompetenz für eine eigenständige EU-Infrastrukturpolitik besteht nicht, die - grundsätzlich raumgreifende - Wirkungsmacht der EU-Grundfreiheiten trägt angesichts des allfälligen Rechtfertigungspotentials der Gemeinwohlaufgabe Infrastruktur nicht sehr weit, und diejenige des EU-Beihilferechts ist durch eine relativierende Praxis von Gerichtshof und Kommission kupiert. In dieser Begrenzung kommt nicht nur der infrastrukturpolitische Autonomieanspruch der Mitgliedstaaten zum Ausdruck, sondern auch die Anerkennung der Daseinsvorsorge als eigenständiger Verfassungswert der Union.

18) Die infrastrukturrelevante Determinierungswirkung des Unionsrechts ergibt sich daher vor allem daraus, dass sein prinzipieller Anwendungsanspruch die Wahrnehmung der Infrastrukturverantwortung, die grundsätzliche Sache der Mitgliedstaaten bleibt, der Aufsicht von Kommission und Gerichtshof unterstellt.

\section{Infrastruktur im Völkerrecht}

19) Da Infrastruktur ortsgebunden ist, unterliegt ihre Errichtung und Nutzung grundsätzlich den allgemeinen Regeln des Völkerrechts über die Nutzung von Staatsgebiet und die Zulässigkeit extraterritorialer Staatsgewalt.

20) Als Gegenstand völkerrechtlicher Verträge finden sich sowohl der gemeinsame Betrieb von Infrastruktur und Infrastrukturregime, als auch die Koordinierung der grenzüberschreitenden Nutzung nationaler Infrastrukturen.

21) Auch das Internet als Basisinfrastruktur der modernen Kommunikationsgesellschaft wird gegenwärtig vor allem aus der Warte der allgemeinen völkerrechtlichen Regeln gesehen und mit ihnen „,bearbeitet“. 


\section{Schluss}

22) Der Zustand der Infrastruktur und Infrastrukturdienste ist ein wesentliches Indiz für den Zustand eines politischen Gemeinwesens, seiner Volkswirtschaft und seiner Verfassungskultur.

23) Angesichts der allgegenwärtigen Entgrenzung politischer Gemeinschaft wird die „Zukunftsfähigkeit“ des Rechts netzgebundener Infrastrukturen vor allem durch internationale Lösungen sicherzustellen sein, während die Regeln über punktförmige Infrastrukturen ihre „Zukunftsfähigkeit“ auch im örtlichen Rahmen unter Beweis stellen können. 

Vierter Beratungsgegenstand:

\title{
Die Anforderungen an ein zukunftsfähiges Infrastrukturrecht
}

\author{
2. Referat von Professor Dr. Hinnerk Wißmann, Münster*
}

Inhalt

Seite

I. Einführung. . . . . . . . . . . . . . . . . 370

1. Ausgangspunkt: Infrastruktur als Herausforderung des Öffentlichen Rechts . . . . . . . . . . . . . . . 370

2. Begriff - Gang der Untersuchung - Ziel . . . . . . . . . . 372

II. Entwicklungslinien und Ordnungsmodelle des Infrastrukturrechts.................... 377

1. Infrastrukturen als Signum moderner Staatlichkeit . . . . . 377

2. Ausdifferenzierung der Zielvorgaben und Regelungsmodelle . . . . . . . . . . . . . . 379

III. Der Verbund von Phasen im Infrastrukturrecht . . . . . . . . 387

1. Planung: Konzeption und Entscheidung . . . . . . . . . . 387

a) Infrastrukturinitiative - Infrastrukturschaffungspflichten . . . . . . . . . . . . . 387

aa) Ausgangslage und Bestand rechtlicher Regelungen . 387

bb) Keine allgemein-ungeschriebenen Infrastrukturschaffungspflichten . . . . . . . . . . . . . 390

b) Planung und Entscheidungsverfahren . . . . . . . . . . . . . 393

aa) Die Rolle des Planungsrechts. . . . . . . . . . . . 393

bb) Entscheidungsverfahren: Planfeststellung . . . . . . 395

cc) Insbesondere: Modifizierungen für öffentliche Infrastrukturen . . . . . . . . . . . . . . . . . . . . . . . 398

dd) Strukturelle Überlastung . . . . . . . . . . . . . . . 399

\footnotetext{
* Ich danke meinen Mitarbeitern in Bayreuth und Münster, namentlich Dr. Boas Kümper, für die treue Unterstützung in der Vorbereitung. Einer frühen Diskussion des Konzepts in einem Kreis von Kolleginnen und Kollegen verdanke ich viel. Christian Bumke und Andreas Voßkuhle haben am Ende durch Kritik und Zuspruch die Fertigstellung des Textes unterstützt.
} 
2. Bereitstellung . . . . . . . . . . . . . 400

a) Grundsatz der Typenwahlfreiheit - Herausforderung Vertragsgestaltung. . . . . . . . . . . . . 400

b) Insbesondere: Private Bereitstellung von Infrastruktur als Vorleistung privilegierter Nutzung - Das Problem von Dienst und Netz . . . . . . . . . . . . . . 402

aa) Anreize für private Investitionen in öffentliche Infrastrukturen . . . . . . . . . . . . . . 402

bb) Verpflichtung zur Öffnung der Infrastruktur für Wettbewerber . . . . . . . . . . . 403

3. Pflege der Infrastruktur . . . . . . . . . . . . . . 405

4. Zusammenfassung: Infrastrukturrecht als Verbundrecht Infrastrukturverwaltung als Dauerverwaltung . . . . . 408

IV. Insbesondere: Das Verhältnis von Verwaltungsarbeit und Gesetz im Infrastrukturrecht . . . . . . . . . . . . . . 411

1. Infrastrukturrecht als Recht politischer Gestaltung . . . . 411

2. Infrastrukturentscheidungen in der verfassungsrechtlichen Funktionengliederung . . . . . . . . . . . . . 412

a) Gewaltenteilung und Einzelfallgesetz . . . . . . . . . 412

b) Vom Einzelfallgesetz zum integrierten Entscheidungsverbund . . . . . . . . . . . . . . 415

V. Schluss . . . . . . . . . . . . . . . . . . . 421

\section{Einführung}

\section{Ausgangspunkt: Infrastruktur als Herausforderung des Öffentlichen Rechts}

Im Großbegriff Infrastruktur sind Mut und Hybris, Weitsicht und Eigennutz, Innovationskraft und Selbstüberschätzung eng verflochten. Geradezu als ein Signum der Moderne steht „Infrastruktur“ für die Grundvoraussetzungen wie für die Gefährdungen einer Gesellschaft, die sich auf Mobilität, Kommunikation und stetige Erneuerung gründet. ${ }^{1}$

Und so ist das Recht der Infrastrukturen - wie zu zeigen sein wird nicht sektorales Technikrecht oder Modethema, sondern in doppeltem

${ }^{1}$ S. Zweig hat die Verlegung des ersten Unterseetelegraphenkabels über den Atlantik 1858 als „Das erste Wort über den Ozean“ in seine „Sternstunden der Menschheit“ aufgenommen - und zugleich vom schnellen Wechsel zwischen Anerkennung und Verwerfung des Projekts berichtet, Ausgabe von 1964/1989, 154 ff. Vgl. noch unten bei Fn. 29 f. 
Sinn eine Herausforderung unseres Fachs mitten im Zentrum des Staatsund Verwaltungsrechts: Funktionierende Infrastrukturen sind - zum einen - eine Vorbedingung ersten Ranges, soll individuelle Freiheit real und nicht nur als atavistische Phrase möglich sein. Wenn Infrastrukturen gegenwärtig in vielfacher Weise fragil werden, ist deshalb tatsächlich über die Architektonik der Bürgergesellschaft im Verfassungsstaat zu verhandeln. ${ }^{2}$ Dabei stoßen nun - zum anderen - die einschlägigen Ordnungsmodelle, mit denen komplexe Vorhaben in technischer, finanzieller und politischer Hinsicht gesteuert werden sollen, zunehmend an Grenzen: ${ }^{3}$ Seit Stuttgart 21 ist allseits bekannt, dass bei der Entscheidung über Großvorhaben selbst aufwendigste Verwaltungs- und Gerichtsverfahren vielfach weder verbindliche noch befriedende Funktion haben; ${ }^{4}$ das Vergaberecht steht geradezu absurden Unzulänglichkeiten scheinbar hilflos gegenüber, wenn Infrastrukturen durch private Unternehmen bereitgestellt werden sollen (nichts anderes gilt freilich für das eigenhändige staatliche Management von Großvorhaben); 5 schließlich weisen die Überflutung von Bahnstrecken oder durch Hitze aufgerissene Autobahnen augenfällig darauf

\footnotetext{
${ }^{2} \mathrm{Zu}$ Integration als Partnerbegriff der Infrastruktur D. van Laak Archiv für Begriffsgeschichte, Band 41 (1999), 280 (290 ff.).

${ }_{3}$ Zur Verbindung von Wandel der Staatlichkeit und verwaltungsrechtswissenschaftlicher Binnenmodernisierung stellvertretend $A$. Voßkuhle Beteiligung Privater an der Wahrnehmung öffentlicher Aufgaben und staatliche Verantwortung, VVDStRL 62 (2003), 266 (286 ff., 307 ff.); E. Schmidt-Aßmann Das allgemeine Verwaltungsrecht als Ordnungsidee, 2. Aufl. 2006, Rn. 1/50 ff.; C. Franzius Gewährleistung im Recht, 2009, 134 ff., 566 ff.; R. Pitschas in: Ziekow (Hrsg.) Wandel der Staatlichkeit und wieder zurück, 2011, 255 (256 ff.); aus staatsrechtlicher Perspektive zuletzt $O$. Lepsius Funktion und Wandel von Staatsverständnissen, Staat Beiheft 21 (2013), 37 (50 ff.).

${ }^{4}$ Übergreifend zuletzt $J$. Saurer DVB1. 2012, 1082 (1086 ff.); K. Waechter/Th. Mann Großvorhaben als Herausforderung für den demokratischen Rechtsstaat, VVDStRL 72 (2013), 499 ff., 544 ff.; breite Darstellung der Rechtslage bei J. Ziekow Neue Formen der Bürgerbeteiligung? Planung und Zulassung von Projekten in der parlamentarischen Demokratie, Gutachten D zum 69. DJT, 2012, D 26 ff., zusammenfassende Defizitanalyse D 61 ff. Vgl. zur aktuellen Diskussion weiter bei Fn. 88, 91. Man wird daran erinnern dürfen, dass in den 1980er Jahren z.B. die Genehmigung von atomaren Endlagern und Flughafenerweiterungen ebenfalls nicht friedensstiftend durch Genehmigungen abgeschlossen wurde, vgl. insoweit für die atomaren Endlager J. Listl DVBl. 1978, 10 ff.; für Flughäfen $V$. Henle UPR 1982, 215 ff., 253 ff.

${ }^{5}$ Vgl. W. Durner in: Sommermann/Magiera (Hrsg.) Daseinsvorsorge und Infrastrukturgewährleistung, 2009, 73 (81 f.). Kritisch zur Entwicklung des Vergaberechts S. Broß VergabeR 2011, 797 (801 f.). Zu den Kostenexplosionen bei Großprojekten der öffentlichen Hand vor dem Hintergrund z.B. der Elbphilharmonie Hamburg oder des BER Flughafen Berlin-Brandenburg etwa Th. Thierau BauR 2013, 673 ff.; K.-R. Wagner BauR 2013, 1596 ff.; vgl. weiter bei Fn. 107.
} 
hin, dass das begleitende (Ordnungs-)Recht die Pflege bestehender Infrastrukturen - eine Megaaufgabe der unmittelbaren Zukunft - nicht verlässlich bewältigt. ${ }^{6}$

In all diesem bestimmen fein ausgearbeitete Fachdiskussionen das Feld, die in den unterschiedlichen Sektoren mit immer höherem Tempo eigenen Pfaden folgen; die Ungleichzeitigkeit der europarechtlichen Integration steigert die Unübersichtlichkeit nochmals in immenser Weise. Verfassungsrechtliche Vorgaben und verwaltungsrechtliche Systembildung erscheinen insgesamt als Getriebene statt als Taktgeber des Geschehens.

\section{Begriff - Gang der Untersuchung-Ziel}

$\mathrm{Zu}$ fragen ist angesichts dieser ersten Problembeschreibung zunächst, ob sich überhaupt ein abgegrenzter Bereich „Infrastruktur“ identifizieren lässt, der für gleiche Fragen gleiche Antworten ermöglicht, die sich von denen anderer Rechtsgebiete unterscheiden. Infrastruktur ist ein vergleichsweise junger Begriff, der Anleihen aus der Ingenieurstechnik, der Ökonomie wie der Soziologie und der (militärischen) Bedarfsplanung nimmt. ${ }^{7}$ Von der wortwörtlichen Verwendung im Eisenbahnbau des

\footnotetext{
${ }^{6}$ Vgl. zuletzt Gesetzesantrag der Bundesländer Sachsen und Bayern zum „Entwurf eines Gesetzes zur Beschleunigung von Hochwasserschutzmaßnahmen (Hochwasserschutzbeschleunigungsgesetz - HWSBG)“, BRat-Drs. 568/13. Vor allem in diesem Feld ist die Finanzierung der Infrastrukturen zuletzt als dringliches Problem erkannt worden, vgl. den Bericht der von K.-H. Daehre geleiteten Kommission „Zukunft der Infrastrukturfinanzierung", 2012, 17 f., 38-40, 46, 105 ff., abzurufen unter http://www. bundesrat.de/DE/gremien-konf/fachministerkonf/vmk, sowie der von $K$. Bodewig geleiteten Kommission „Nachhaltige Verkehrsinfrastrukturfinanzierung“, 2013, abzurufen unter http://www.bundesrat.de/cln_350/nn_8794/DE/gremien-konf/fachministerkonf/ vmk, 9-12, 25, $29 \mathrm{ff}$.

7 Aufbereitung der Begriffsgeschichte bei $D$. van Laak Archiv für Begriffsgeschichte, Band 41 (1999), 280 ff., mwN; ausführlich ders. Imperiale Infrastruktur, 2004, 17 ff.; vgl. auch $K .-W$. Schatz Zur Entwicklung des Begriffs Infrastruktur, in: Berger (Hrsg.) Wettbewerb und Infrastruktur in Post- und Telekommunikationsmärkten, 1996, 122 (124 ff.). Wichtig für die allgemeine Debatte war vor allem die wirtschaftswissenschaftliche Aufarbeitung und Theoriebildung, in Deutschland angestoßen maßgeblich von $R$. Jochimsen Über „Infrastrukturen“ als Voraussetzungen einer funktionsfähigen Volkswirtschaft, in: Kindermann (Hrsg.) Kulturen im Umbruch, 1962, 29 ff. Vgl. zur ökonomischen Perspektive G. Aberle Art. Infrastruktur, Staatslexikon, 1987, Sp. 78 ff.; Th. Schulze Infrastruktur als politische Aufgabe, 1993, $40 \mathrm{ff}$; zuletzt im Überblick U. Baßeler/J. Heinrich/B. Utecht Grundlagen und Probleme der Volkswirtschaft, 19. Auflage 2010, 848; P. Samuelson/W. Nordhaus Volkswirtschaftslehre, 4. Auflage 2010, 747; für den internationalen Zugriff T. Henckel/W. McKibbin The economics of infrastructure in a globalized world: issues, lessons and future challenges, 2010, insb. $3 \mathrm{ff}$.
} 
19. Jahrhunderts über die allgemeine planerische Nutzung hat er eine stramme Entwicklung hin zu einem viel genutzten Leitbegriff durchlaufen, der am Ende neben dem Bereich der physischen Einrichtungen Straße, Schiene, Kanal - zu allen Dingen gehört, weil alles und jedes seinen „Unterbau“, seine Voraussetzungen benötigt. ${ }^{8}$

Für das Recht ist zu verzeichnen, dass es an dieser allgemeinen Begriffsgeschichte eher passiv beteiligt war und sich so bis in die Gegenwart keine klare Vorstellung vom Inhalt des Begriffs durchgesetzt hat. Erste Verwendungen im europäischen, transatlantischen und deutschen Rechtskreis nach dem 2. Weltkrieg blieben zunächst noch blass und zufällig. ${ }^{9}$ Vor allem durch die Karriere der Planungsidee wurde der Begriff dann mobilisiert ${ }^{10}$ und ab 1969 zunehmend allgemein genutzt. ${ }^{11}$ Verfas-

${ }^{8}$ Zur „ganzheitlichen“ Dimension des Begriffs, der neben quantitativen auch qualitative Aspekte einbezieht und damit insbesondere auch in den Umweltwissenschaften Beachtung gefunden hat, U. E. Simonis Zur inhaltlich-systematischen Deutung des Begriffs Infrastruktur, Zeitschrift für Ganzheitsforschung, N. F. 27 (1983), 119 ff. Vgl. beispielsweise zur Vielschichtigkeit der „Kommunikationsinfrastruktur“ $K$.-H. Ladeur Die Kommunikationsinfrastruktur der Verwaltung, in: Hoffmann-Riem/Schmidt-Aßmann/ Voßkuhle (Hrsg.) Grundlagen des Verwaltungsrechts, Band II, 2. Aufl. 2012, § 21, insb. Rn. 86 f.; zur „komplexen Infrastruktur kollektiver Ordnungsbildung“ ders. Staat 48 (2009), 163 (169).

9 Auf Bundesebene erstmals in Titel 601 des Kapitels 1402 des Bundeshaushaltsplan für das Rechnungsjahr 1957, Einzelplan 14 für den Geschäftsbereich des Bundesministers für Verteidigung, 2161.

${ }^{10}$ Vgl. zeittypisch H. Filbinger Probleme der Infrastruktur in der Raumordnung, Staatsanzeiger für Baden-Württemberg, 9.10.1965, 1 f.; E. Egner Art. Raumwirtschaftspolitik, in: Handwörterbuch der Sozialwissenschaften, Band 8, 1964, 694 (698).

${ }^{11}$ Erste normative Verwendung im Bundesrecht in $\S 1$ Abs. 1 des Gesetzes über die Gemeinschaftsaufgabe „Verbesserung der regionalen Wirtschaftsstruktur" vom 6.10. 1969, BGB1. I 1861 („Förderung des Ausbaus der Infrastruktur“, § 1 Abs. 1 Nr. 2; „Infrastrukturmaßnahmen“, § 1 Abs. 3); dann $\S 5$ Abs. 3, Abs. 6 f. des Durchführungsgesetzes zum Gesetz über einen Ausgleich für Folgen der Aufwertung der Deutschen Mark auf dem Gebiet der Landwirtschaft (,Agrar- und Infrastruktur“, in diesem Doppelsinn auch bereits 1968 in BT-Drs. V/2672 (Entwurf eines Agrarstrukturgesetzes)), BGBl. I 1970, 676 ff. (677 f.); $\$ 3$ Abs. 3 Nr. 2 c) Städtebauförderungsgesetz (,infrastrukturelle Erschließung“), BGBl. I 1971, 1125 ff. (1128) (mit allgemeiner Verwendung des Begriffs in BT-Drs. VI/510, 25 f.), gleichlautend noch heute in $\S 136$ Abs. 3 Nr. 2c) BauGB; Art. 19 und 28 des Gesetzes zum Assoziierungsabkommen der EWG mit Madagaskar („Infrastruktur“, „wirtschaftliche und soziale Infrastrukturanlagen“), BGBl. II 1970, 521 ff. (542, 548). Vgl. für die Rechtsprechung 1974 BVerfGE 38, 258 (270 f.) - Magistratsverfassung: „Einrichtungen der Energie- und Wasserversorgung, des Nahverkehrs, der Abfallbeseitigung, der Krankenhäuser, Altenheime und Kindergärten wie für sonstige Maßnahmen zum Ausbau der örtlichen ,Infrastruktur““. 
sungsrechtlich ist er zwar nicht in das Grundgesetz promoviert worden, wohl aber im AEUV zu finden. ${ }^{12}$

Im geltenden Gesetzesrecht wird der Terminus „Infrastruktur“ prominent vor allem im Recht der Raumordnung verwandt, schon dort allerdings mit einer beachtenswerten Weite, wenn etwa von bestimmten „Infrastrukturen der Daseinsvorsorge“ gesprochen wird, aber eben auch in allgemeiner Weise von „sozialer“, von „kritischer“ oder „wirtschaftsnaher" Infrastruktur, die zu gewährleisten, zu bündeln, zu entwickeln, zu schützen ist. ${ }^{13}$ Zahlreiche Fachgesetze - von TKG, EnWG und GWB über das SGB bis hin zum Wertpapierhandelsgesetz - nutzen den Terminus gleichfalls in durchaus unterschiedlicher Weise. ${ }^{14}$ Zugleich findet er sich in

12 V. a. Art. 170, nachrangig 171, 177 AEUV, eingeführt 1993 durch den Vertrag von Maastricht, vgl. zur Entwicklung Ch. Calliess in ders./M. Ruffert (Hrsg.) EUV/AEUV, 4. Aufl. 2011, Art. 170 Rn. 1.

${ }_{13}$ Alles in $\S 2$ Abs. 2 Nr. 3 f. ROG (Grundsätze der Raumordnung). Infrastruktur wird maW sowohl als Teil bestimmter Sachgebiete („Soziale Infrastruktur“, § 2 Abs. 2 Nr. 3 ROG) als auch als eigenständige Materie verstanden, vgl. § 2 Abs. 2 Nr. 1 ROG, wonach im gesamten Bundesgebiet ,ausgeglichene soziale, infrastrukturelle, wirtschaftliche, ökologische und kulturelle Verhältnisse“ anzustreben sind. Ähnlicher Selbststand des Begriffs in $\S 8$ Abs. 5 Nr. 3 ROG, wo Infrastruktur als Verkehrs- und Versorgungs-/ Entsorgungsinfrastruktur verstanden wird. Es ist darauf hinzuweisen, dass das ROG in seiner Ursprungsfassung 1965 den Begriff (ebenso wie die ökologischen Verhältnisse) noch nicht kannte (BGBl. I 1965, 306), sondern er erst 1989 (§ 1 Abs. 1, BGBl. 1989, 1461 (Neubekanntmachung), vgl. zur Begründung BT-Drs. 11/3916, 9 ff.) bzw. 1998 (§ 2 Abs. 2, § 7 Abs. 2, BGBl. 1997, 2081) Eingang in das Gesetz gefunden hat.

${ }^{14}$ Eher gegenständliche Verwendung in $\S 19$ Abs. 2 Nr. 3 GWB (,Zugang zu eigenen Netzen oder anderen Infrastruktureinrichtungen“), § 3 Nr. 9b) TKG (,Netzinfrastruktur“), § 3 Nr. 32b) TKG (,Zugang zu physischen Infrastrukturen wie Gebäuden, Leitungsrohren und Masten“), § 77a TKG (,Gemeinsame Nutzung von Infrastrukturen durch Betreiber öffentlicher Telekommunikationsnetze: (1) Die Bundesnetzagentur kann die gemeinsame Nutzung von Verkabelungen oder Kabelkanälen (...) anordnen“), $\S 13 \mathrm{a}$ Abs. 2 Nr. 3 BauGB (,Verwirklichung von Infrastrukturvorhaben“), ähnlich $\S 136$ Abs. 3 BauGB, § 11 Abs. 3 BauNVO, § 11 Abs. 1 AEG (,Abgabe und Stilllegung von Eisenbahninfrastruktureinrichtungen: (1) Beabsichtigt ein öffentliches Eisenbahninfrastrukturunternehmen die dauernde Einstellung des Betriebes einer Strecke, eines (...) Bahnhofs oder die mehr als geringfügige Verringerung der Kapazität einer Strecke, (...)"); als allgemein-offene Sammelbezeichnung in § 128 SGB XI („Aufbau der technischen und organisatorischen Infrastruktur"), § 2 Abs. 3 Nr. 4 TKG („Investitionen und Innovationen im Bereich neuer und verbesserter Infrastrukturen"), § 21 Abs. 1 Nr. 4 TKG (,Investitionen in Infrastruktureinrichtungen“), § 40 Abs. 2 Nr. 2 TKG (,Wettbewerb im Bereich Infrastruktur“); $\$ 77$ b) („Alternative Infrastrukturen: (...) Einrichtungen, die zum Auf- und Ausbau von Netzen der nächsten Generation genutzt werden können“), § 3 Nr. 5a BImSchG („Anlagen einschließlich gemeinsamer oder verbundener Infrastrukturen“); spezifische Verwendung dagegen etwa in § 291a SGB V (,Telematikinfrastruktur“ als Zusammenfassung von „Informations-, Kommunikations- und 
Gesetzen überhaupt nicht, die wir intuitiv dem Sachbereich der Infrastruktur zuordnen (wie etwa dem NABEG ${ }^{15}$ ).

Es ist nun allerdings kein Zufall oder bloße Nachlässigkeit, warum ein präziser Rechtsbegriff der Infrastruktur offensichtlich nur schwer gebildet werden kann. Denn er bezieht sich nach dem hier vertretenen Verständnis nicht auf einen bestimmten, festliegenden Gegenstand, sondern auf eine spezifische Fragestellung. Dem Recht der öffentlichen Infrastrukturen geht es um die Planung (d.h. Konzeption und Entscheidung), Bereitstellung und Pflege von Einrichtungen, die unmittelbar von der Allgemeinheit genutzt werden können oder für weitere Dienste zur Verfügung stehen, die sich grundsätzlich an die Allgemeinheit richten. Infrastruktur kann damit Straße und Schiene umfassen, die Netze der Telekommunikation, der Energie- wie Wasserversorgung, ebenso wie „stationäre“ Einrichtungen Flughäfen, Krankenhäuser und Schulen. ${ }^{16}$ Und während bei der Straße das „Netz“ selbst bereits Infrastruktur ist, ergibt sich etwa schon bei

\footnotetext{
Sicherheitsinfrastruktur" in Bezug auf die elektronische Gesundheitskarte), in $§ 1$ Kreditwesengesetz (,Nutzung von Infrastrukturen, die darauf abzielen, Latenzzeiten zu minimieren" als Beispiel für Finanzdienstleistungen), ähnlich $\S 2$ Wertpapierhandelsgesetz, § 3 Abs. 3 Nr. 3 TKG (,infrastrukturbasierter Wettbewerb“), § 90a Investmentgesetz („Infrastruktur-Sondervermögen“), in den landesrechtlichen Geodateninfrastrukturgesetzen, die den Begriff als „Infrastruktur aus Geodaten, Metadaten und Geodatendiensten" usw. definieren (z.B. Art. 3 Abs. 6 BayGDIG). Durchaus heterogen auch die Verwendung durch das Bundesverfassungsgericht, vgl. etwa BVerfGE 38, 258 (270) - Magistratsverfassung (offene Formel zu kommunalen Infrastrukturen, s.o. Fn. 11); 110, 177 (192 f.) - Freizügigkeit (,,infrastrukturelle Folgelasten“: u.a. Herstellung von Einrichtungen der Kinderbetreuung, der öffentlichen Versorgung und Entsorgung); 120, 1 (39) - Gewerbesteuer Freiberufler (Infrastrukturlasten bei Ansiedlung von Gewerbebetrieben); 125, 39 (86) - Adventssonntage (Infrastruktur: Energieversorgung und Sicherung der Mobilität); 125, 260 (314) - Telekommunikationsverkehrsdaten (Telekommunikationsinfrastruktur); referierend 127, 165 (168 f.) - Informationsbeschaffung (u.a. „Bildungsinfrastruktur“ sowie Krankenhäuser, „ländliche Infrastruktur", „sonstige Infrastrukturinvestitionen“).

${ }_{15}$ Gleiches gilt für das Planvereinheitlichungsgesetz vom Juni 2013, BGBl. I 1388, mit der Einführung der ,frühen Öffentlichkeitsbeteiligung" nach $\S 25 \mathrm{Abs} .3 \mathrm{VwVfG}$.

16 Vgl. für einen offenen Begriff BVerfGE 38, 258 (270 f.) - Magistratsverfassung, s.o. Fn. 11, vgl. auch BVerfGE 127, 165 (168 f.) - Informationsbeschaffung. Differenzierend unter Abgrenzung vom Begriff der öffentlichen Versorgung J.-C. Pielow Grundstrukturen öffentlicher Versorgung, 2001, 21 ff. Weit unter Einschluss etwa von Schulund Universitätsgebäuden zuletzt auch J. Kühling DVBl. 2013, 1093 (1094 f.); entsprechend für die Schweiz M. Lendi UPR 2011, 422 (422); enger etwa W. Erbguth DVBl. 2009, 265 (265 f.). Nicht erfasst sind alle nur faktisch der Öffentlichkeit zugänglichen Einrichtungen, wie etwa die von privaten Betrieben geprägten ,zentralen Versorgungsbereiche“, vgl. dazu zuletzt H.-J. Koch Die Verwaltung 45 (2012), 233 (234); U. Battis DVB1. 2011, $196 \mathrm{ff}$.
} 
Schiene, Energie und Telekommunikation erst durch eine integrierte Betrachtung, die die Kopplung der Einrichtung mit den Diensten einbezieht, eine sinnvolle Perspektive. ${ }^{17}$ Freilich gehören die Einzelheiten von Zugang und Entgelt, Versorgungssicherheit und Datenschutz zu einem „Infrastrukturrecht II“, das an die hier zu behandelnden Fragen erst anschließt. ${ }^{18}$

Insgesamt ist also der Sachbereich der Infrastruktur begrifflich per se nicht abschließend bestimmt, weder in Hinblick auf Aufgaben, Akteure noch Ordnungsmodelle: Der Bestand und die Form allgemein zugänglicher Einrichtungen sind offen und grundsätzlich gestaltbar. ${ }^{19}$ Anders gewendet: Für die rechtliche Debatte ist Abstand zu halten von sektoralen Begriffsverengungen („Infrastruktur als Teil des Rechts der Netze“) ebenso wie von voreiligen ökonomischen Normativismen, öffentliche Infrastrukturen seien Voraussetzung und zugleich Ausnahme eines Marktoder Wettbewerbsgeschehens. ${ }^{20}$

Die sachnotwendig bestehende Vielschichtigkeit des Begriffs zeigt an so ein erstes Zwischenergebnis -, dass es ein Infrastrukturrecht (samt einheitlicher Erwartungen daran) kaum geben kann. Die Rede vom Recht der öffentlichen Infrastrukturen ist vielmehr eine Chiffre, unter der allerdings grundlegende Herausforderungen des Verwaltungsrechts für unsere Zeit verhandelt werden können: Mit dieser Gesamtperspektive möchte ich zunächst darlegen, dass Infrastrukturen und ihre Regelungsmodelle in

${ }_{17}$ Die Verbindung beider Bereiche prägt auch die neueren Begriffsbildungen in der Rechtswissenschaft, vgl. insbesondere G. Hermes Staatliche Infrastrukturverantwortung, 1998, insb. 330 ff.; zuletzt entsprechend W. Hoffmann-Riem Rechtsformen, Handlungsformen, Bewirkungsformen, in: ders./Schmidt-Aßmann/Voßkuhle (Hrsg.) Grundlagen des Verwaltungsrechts, Band II, 2. Aufl. 2012, § 33 Rn. 94 („Infrastrukturregulierung"); für eine Verbindung von Einrichtung und Dienst grundsätzlich auch $W$. $G$. Leisner WiVerw 2011/2, 55 (70 f.). Tendenziell enger unter Ausschluss der Nutzung ist der europarechtliche Begriff der Infrastruktur, vgl. Ch. Calliess in: ders./Ruffert (Hrsg.) EUV/AEUV, 4. Aufl. 2011, Art. 170 Rn. 7; vgl. auch die Beiträge des Abschnitts „Infrastrukturrecht“" zu Telekommunikation, Energie und „Transportrecht“ von J. Kühling, M. Ludwigs und M. Knauff in Ruffert (Hrsg.) Europäisches sektorales Wirtschaftsrecht (EnzEur Band 5), 2013.

18 Vergleiche zu Schnittstellen unten bei Fn. 112, $113 \mathrm{ff}$., $125 \mathrm{ff}$.

${ }_{19}$ Ebenso J. Kühling DVBl. 2013, 1093 (1094); vgl. zur Bandbreite der Ausgestaltung bereits Hermes Infrastrukturverantwortung (Fn. 17), 342 ff., 390 ff. Entsprechend Pielow Grundstrukturen (Fn. 16), 311, der für das Kriterium der Öffentlichkeit auf den allgemeinen Adressatenkreis abstellt.

${ }^{20}$ Klassisch für eine sektorale Verwendung: Infrastruktur als Begriff der militärischen Bedarfsplanung, so etwa heute noch im „Bundesamt für Infrastruktur, Umweltschutz und Dienstleistungen der Bundeswehr (BAIUDBw)“; zur ökonomischen Sichtweise etwa $S c h a t z$ (Fn. 7), passim. 
signifikanter Weise mit der Entwicklung des modernen Staats verbunden sind. Die dabei entstandenen Infrastrukturteilrechtsordnungen sind bis heute deutlich unterscheidbar, und geben so Zeugnis von den regulativen Wellenbewegungen, in die ausdifferenzierte Zielvorgaben ebenso integriert wurden wie die Fortschreibung des verwaltungsrechtlichen Instrumentariums (II.). Im Anschluss soll gezeigt werden, dass sich das Infrastrukturrecht in den Phasen von Planung, Bereitstellung sowie Nutzung und Pflege von Infrastruktur modellieren lässt und diese Phasen in stärkerer Weise als bisher geschehen als auf Dauer angelegtes Verbundsystem aufeinander bezogen werden müssen (III.). Durch den notwendig politischen Charakter von Infrastrukturentscheidungen ergeben sich abschließend Folgerungen für das Verhältnis von Verwaltungsverfahren und Parlamentsgesetz (IV.)

\section{Entwicklungslinien und Ordnungsmodelle des Infrastrukturrechts}

\section{Infrastrukturen als Signum moderner Staatlichkeit}

Infrastrukturen und Infrastrukturrecht bilden ein spezifisches Strukturmerkmal moderner Staatlichkeit. $\mathrm{Zu}$ beobachten ist eine historisch gewachsene Schichtung verschiedener regulativer Grundannahmen und Leitmodelle, die sich nebeneinander bis in die Gegenwart erhalten haben.

Ein Fehlschluss wäre demgegenüber die Annahme, erst die Gegenwart hätte mit dem Begriff auch den Gegenstand oder die Rechtsprobleme der Infrastruktur erfunden. Selbstverständlich war es gerade ein Kennzeichen schon des frühneuzeitlichen Staates, dass er Infrastruktur schaffte oder schaffen ließ: Durch die technisch-kartographische Erfassung des Herrschaftsgebiets, den Bau von Kanälen, Straßen, aber etwa auch die Einrichtung von Schulen wird Stück für Stück die mittelalterliche „Herrschaft vom Sattel aus“ überwunden. ${ }^{21}$ Die abstrakten Herrschaftsgebiete wurden mit anderen Worten nicht allein durch Reglement und Gesetz, sondern erst durch die konkrete, planmäßig voranschreitende ErschlieBung des Territoriums, eben durch Infrastruktur, Staaten in unserem Sinn. ${ }^{22}$

${ }^{21}$ Zur Lage im Mittelalter G. Althoff Die Ottonen. Königsherrschaft ohne Staat, 2. Aufl. 2005, insb. 230 ff., 243 ff.; vgl. begriffsprägend $O$. Brunner Land und Herrschaft, 4. Aufl. 1959, 180 ff. Zur Rolle der Städte für die Entwicklung von Infrastruktur M. Stolleis Geschichte des Sozialrechts, 2003, 13 ff., in Bezug auf Armenversorgung und Krankenwesen.

22 Vgl. dazu umfassend D. Blackbourn Die Eroberung der Natur, 2006, vor allem zum Bereich von Flüssen, Kanälen und Entwässerung; insbesondere zur Entwicklung der Mobilität C. Merki Verkehrsgeschichte und Mobilität, 2008, vor allem 76 ff.; zur all- 
In diesem langgestreckten Prozess bildet das 19. Jahrhundert einen entscheidenden, auf die Gegenwart weisenden Einschnitt. Rechtsstaatliche Sicherungen, ${ }^{23}$ Gewerbefreiheit ${ }^{24}$ und Schutz des geistigen Eigentums ${ }^{25}$ schufen einen neuen Rahmen für technische Entwicklungen, die sich nun in neuer Dynamik entfalteten. ${ }^{26}$ Neben der linearen Zunahme von Geschwindigkeit und Produktivität, für die die Eisenbahn steht, ${ }^{27}$ führte vor allem der Siegeszug der Elektrizität zu einem grundlegenden Wandel: ${ }^{28}$ Seit den 1830er Jahren sind die Städte und seit den 1860er Jahren die Kontinente durch die Telegraphie verbunden und damit in einen gemeinsamen, auf Gleichzeitigkeit basierenden Erfahrungsraum gesetzt worden. ${ }^{29}$ Die verlässliche Vernetzung ganzer Territorien, ja im Grunde schon damals der ganzen bekannten Welt, ist seitdem ein typisches Kennzeichen

mählichen Entwicklung des allgemeinen Schulwesens im Flächenstaat $H$. Wißmann in: Reimer (Hrsg.) Homeschooling - Bewährung oder Bedrohung des freiheitlichen Rechtsstaats?, 2012, 17 (20 ff., 31 ff.). Aus wirtschaftswissenschaftshistorischer Sicht Schulze Infrastruktur (Fn. 7), $71 \mathrm{ff}$.

${ }^{23}$ Zur begriffsgeschichtlichen Entwicklung E.-W. Böckenförde FS Arndt, 1969, 53 ff.; H. Hofmann Der Staat 34 (1995), 1 ff. Exemplarisch zur praktischen Entwicklung E.-R. Huber Deutsche Verfassungsgeschichte seit 1789, Band I, 2. Aufl. 1967, 617; Band II, 2. Aufl. 1968, 835 f.; Band III, 2. Aufl. 1970, 111 f., 1071 ff. Zur allmählichen Durchsetzung der Gesetzesbindung und der rechtswissenschaftlichen Formierung des Verwaltungsrechts im Überblick H. Wißmann Generalklauseln, 2008, $56 \mathrm{ff}$.

${ }^{24}$ Zur Entwicklung B. Vogel Allgemeine Gewerbefreiheit, 1983, insb. 165 ff.; J. Ziekow Freiheit und Bindung des Gewerbes, 1992, $327 \mathrm{ff}$.

${ }^{25}$ E. Wadle Geistiges Eigentum, Band II, 2003, 3 ff.; insbesondere auch zum Schutz des Persönlichkeitsrechts D. Klippel ZNR 4 (1982), 132 ff.; weiter speziell L. Gieseke Vom Privileg zum Urheberrecht, Die Entwicklung des Urheberrechts in Deutschland bis 1845, 1995; L. Pahlow Lizenz und Lizenzvertrag im Recht des Geistigen Eigentums, 2006, 18 ff.; A. K. Schmidt Erfinderprinzip und Erfinderpersönlichkeitsrecht im deutschen Patentrecht von 1877 bis 1936, 9 ff.

${ }^{26}$ Zum Zusammenhang der Faktoren L. Jellinghaus Zwischen Daseinsvorsorge und Infrastruktur, 2006, insb. 14.

${ }^{27} \mathrm{Zu}$ den ersten, in der Regel auf private Unternehmen bezogenen Regelungen vgl. das preußische „Gesetz über die Eisenbahn-Unternehmungen“ vom 3.11.1838, GS 305, sowie das preußische Enteignungsgesetz von 1874, GS 221, dazu G. Püttner FS Blümel, 1999, 467 ff.; S. Stamm Eisenbahnverfassung und Bahnprivatisierung, 2010, 29 ff.; vgl. auch K. Bracht Der Bau der ersten Eisenbahnen in Preußen, 1998, 14 ff. Zur späteren Verstaatlichung unten bei Fn. $40 \mathrm{ff}$.

${ }^{28}$ Knapper Überblick über die frühe Entwicklung der Energieversorgung in Deutschland bei Hermes Infrastrukturverantwortung (Fn. 17), 285 ff. Vgl. weiter Fn. 40.

${ }^{29}$ Vgl. T. Standage The Victorian Internet, 1998/2007; skeptisch zu dieser Deutung angesichts der großen technischen Schwierigkeiten in Bezug auf die Verlegung des transatlantischen Telegrafenkabels C. Holtorf Der erste Draht zur neuen Welt, 2013; vgl. aber auch nochmals Zweig Sternstunden (Fn. 1). 
von Infrastruktur, ${ }^{30}$ das neben die Versorgung ,, vor Ort “ tritt - beides bildet schon früh eine reguläre Aufgabe des Infrastrukturrechts. ${ }^{31}$

\section{Ausdifferenzierung der Zielvorgaben und Regelungsmodelle}

Das Infrastrukturrecht muss dabei seit jeher ganz gegenläufige Zielvorgaben bewältigen. Um die wichtigsten Etappen zu nennen: Bereits im frühen Rechtsstaat wurden die Fragen von allgemeinem Bedarf, konkretem unternehmerischem Verwirklichungsinteresse und eventuell notwendiger Enteignung mit dem Ziel allseitiger Rechtssicherheit miteinander verknüpft. ${ }^{32}$ Mit dem Stichwort der räumlichen Gesamtplanung ${ }^{33}$ traten

${ }^{30}$ Auf diese Dimension weist Standage (Fn. 29) mit seinem auf den Telegraphen bezogenen Titel hin, vgl. auch 1 (,Mother of all networks“).

${ }^{31}$ Zur Entwicklung des Verhältnisses von „Democracy \& Public Space“ vgl. am Beispiel von New York und London L. Keller Triumph of Order, 2009. Vgl. zur gegenwärtigen Entwicklung sektorenübergreifend stellvertretend A. Voßkuhle Der Wandel von Verwaltungsrecht und Verwaltungsprozeßrecht in der Informationsgesellschaft, in: Hoffmann-Riem/Schmidt-Aßmann (Hrsg.) Verwaltungsrecht in der Informationsgesellschaft, 2000, 349 (351 ff.); J. Masing FS Wahl, 2011, 147 (156 ff.). Zur Bedeutung von Mobilität insbesondere für die europäische Integration M. Knauff Transportrecht, in: Ruffert (Hrsg.) Europäisches sektorales Wirtschaftsrecht (EnzEur Band 5), 2013, § 6 Rn. 3 ff.; allgemein zum supranationalen und internationalen Kontext J. Saurer DVBl. 2012, 1082 (1084 f.). Als ein Zielpunkt vgl. das europarechtliche Ziel des „Auf- und Ausbaus transeuropäischer Netze" Art. 170 Abs. 1 AEUV, bezogen auf die Bereiche von „Verkehrs-, Telekommunikations- und Energieinfrastruktur“.

32 Prototypisch die Formulierung des Aufopferungsanspruchs in der Einleitung des Allgemeinen Landrechts für die Preußischen Staaten (1794): ,\$. 74. Einzelne Rechte und Vortheile der Mitglieder des Staats müssen den Rechten und Pflichten zur Beförderung des gemeinschaftlichen Wohls, wenn zwischen beyden ein wirklicher Widerspruch (Collision) eintritt, nachstehn. §. 75. Dagegen ist der Staat denjenigen, welcher seine besondern Rechte und Vortheile dem Wohle des gemeinen Wesens aufzuopfern genöthigt wird, zu entschädigen gehalten. "Zur historischen Entwicklung des Instituts der Planfeststellung, ausgehend von $\S 4$ des preußischen „Gesetzes über die Eisenbahn-Unternehmungen“ vom 3.11.1838, GS 305, grundlegend W. Blümel Die Bauplanfeststellung I, 1961; vgl. ferner unter Berücksichtigung weiterer Forschungsergebnisse ders. FS Hoppe, 2000, 3 ff. Konkreter (Primär-) Rechtsschutz entwickelte sich erst langsam in einzelnen Ländern, siehe D. Grimm in: Coing/Wilhelm (Hrsg.) Wissenschaft und Kodifikation des Privatrechts im 19. Jahrhundert, Band IV, 121 (132 f.). Zum Aspekt der Rechtssicherheit in der gegenwärtigen Lage A. v. Arnauld Rechtssicherheit, 2006, speziell zum Planungsrecht 318 ff.; J. Saurer DVBl. 2012, 1082 (1085 f.), dort auch zum historischen Antagonismus zwischen rechtsstaatlicher und demokratischer Steuerung.

${ }_{33}$ Zur Entwicklung der allgemeinen Raumordnung W. Ernst in: Kaiser (Hrsg.) Planung III, 1968, 129 ff.; U. Battis JA 1981, 313 ff.; W. Appold FS Hoppe, 2000, 21 ff. Zur vorgängigen Entstehung des Stadtplanungsrechts und der ersten übergemeindlichen Planungsverbände vgl. etwa $R$. Breuer Die Verwaltung 19 (1986), 305 ff. Vgl. für die aktuelle Lage W. Durner NuR 2009, 373 ff., s. weiter Fn. 41, 51, 83. 
später die Annäherung der Lebensverhältnisse ${ }^{34}$ sowie dann die Einbeziehung der Umweltwirkungen ${ }^{35}$ von Infrastrukturen hinzu. Gerade hier entwickelte sich die Frage nach angemessenen Beteiligungs- und Entscheidungsformen bei Großvorhaben. ${ }^{36}$ Schließlich war und ist parallel stets die Frage nach der technischen Offenheit des Infrastrukturrechts immer wieder neu zu stellen. ${ }^{37}$

34 J. Kersten DVB1. 2006, 942 (943 ff.). Das ROG hat immer ambitionierter die Angleichung der Lebensverhältnisse als Ziel formuliert (mit dem Höhepunkt § 1 Abs. 2 Nr. 6 ROG (1998): Bei der Aufgabenerfüllung der Raumplanung sind „gleichwertige Lebensverhältnisse in allen Teilräumen herzustellen." Vgl. auch § 2 Abs. 2 Nr. 4 ROG (1998)). Das ROG 2009 hat die Verschiedenheit der Situation stärker in den Blick genommen und damit Flexibilisierungen verbunden, vgl. § 2 Abs. 2 Nr. 2 f. ROG (2009); kritisch zur Abschmelzung J. Kersten UPR 2006, 245 ff.

${ }_{35}$ Zur Beziehung von Umweltschutz und Planungsrecht allgemein W. Erbguth Rechtssystematische Grundfragen des Umweltrechts, 1987, 146 ff.; E. Schmidt-Aßmann DÖV 1990, 169 ff.; R. Bartlsperger FS Hoppe, 2000, 127 ff.; zur Beziehung von Umweltschutz und Fachplanung S. Paetow/R. Wahl in: Hansmann/Sellner (Hrsg.) Grundzüge des Umweltrechts, 4. Aufl. 2012, Kap. 4 Rn. 1 ff.; zu Umweltschutzbelangen in der Raumordnung A. Scheidler NVwZ 2010, 19 ff.; O. Rojahn NVwZ 2011, 654 ff. Speziell dann zur strategischen Umweltprüfung $R$. Hendler (Hrsg.) Die strategische Umweltprüfung (sog. Plan-UVP) als neues Instrument des Umweltrechts, 2004; W. Erbguth (Hrsg.) Strategische Umweltprüfung (SUP) - Stand, Rechtsfragen, Perspektiven -, 2006. Zum Stand des Abfallrechts anhand des KrWG (2012) M. Beckmann AbfallR 2012, 142 ff.; J. Klement VerwArchiv 103 (2012), 218, insb. 221 ff. zur „Publifizierung“; zur Entwicklung M. Kloepfer AbfallR 2012, 261 (262 ff.).

${ }^{36}$ Vgl. zu Frühformen der diesbezüglichen Kommunikation der Verwaltung im 19. Jahrhundert $P$. Cancik Verwaltung und Öffentlichkeit in Preußen, 2007, 9 ff.; zum Topos der Partizipation A.-B. Kaiser Die Kommunikation der Verwaltung, 2009, $136 \mathrm{ff}$. Umfassende Problemanalysen für die Gegenwart bei $K$. Waechter Großvorhaben (Fn. 4), 501 ff.; Th. Mann Großvorhaben (Fn. 4), 546 ff.; J. Ziekow Gutachten D zum 69. DJT, 2012; J. Saurer DVB1. 2012, 1082 (1086 ff.). Zur Diskussion im Einzelnen noch bei Fn. 88, 91 .

${ }^{37}$ Stellvertretend zum Problem der Standardsetzung im Recht am Beispiel der Telekommunikation K.-H. Ladeur Der Staat 48 (2009), 163 (172 ff.); s. auch I. Appel Methodik des Umgangs mit Ungewissheit, in: Schmidt-Aßmann/Hoffmann-Riem (Hrsg.) Methoden der Verwaltungsrechtswissenschaft, 2004, 327 (342 ff.). Zum zugrundeliegenden Dualismus der Zielstellungen W. Hoffmann-Riem Recht als Instrument der Innovationsoffenheit und Innovationsverantwortung, in: Hof/Wengenroth (Hrsg.) Innovationsforschung, 2007, 387 (397 ff.); vgl. dann M. Eifert/W. Hoffmann-Riem (Hrsg.) Innovationsfördernde Regulierung, 2009, sowie M. Eifert/W. Hoffmann-Riem (Hrsg.) Innovationsverantwortung, 2011. Zu den Grundkategorien O. Lepsius Risikosteuerung durch Verwaltungsrecht: Ermöglichung oder Begrenzung von Innovationen?, VVDStRL 63 (2004), 264 (278 ff.). In Bezug auf die Konvergenz der Märkte zwischen Kabel/Telefon/ Internet und Kabel/Fernsehen vgl. zuletzt Beschluss des OLG Düsseldorf vom 14.8. 2013, Az. VI - Kart 1/12 (V) zur Fusion im Kabelfernsehen; vgl. dazu allgemeiner T. Fetzer WiVerw 2010, 145 ff. Zum Topos der Netzneutralität C. Franzius N\&R 2012, 
Die angesprochene historische Schichtung der Aufgaben hat das Infrastrukturrecht in regulativen Wellenbewegungen verarbeitet. ${ }^{38}$ In einer stark vergröbernden Gesamtschau folgte in Deutschland und Kontinentaleuropa nach privatwirtschaftlichen Anfängen ${ }^{39}$ eine lange Phase überwiegend staatlicher bzw. kommunaler Eigentätigkeit ${ }^{40}$ und Planung. ${ }^{41}$ Nach

126 (133 ff.) sowie unten Fn. 112; zu Synergievorgaben im Bereich des Breitbandausbaus B. KindlM. Schramm N\&R 2012, 140 ff. Zur Konvergenz zwischen Straßenverkehr und Energieversorgung am Beispiel S. Michaels/Ch. De Wyll R. Ringwald DÖV 2011, $831 \mathrm{ff}$.

${ }^{38} \mathrm{Vgl}$. zum systemtheoretisch angeleiteten Ansatz der reflexiven Modernisierung als Endlosprozess $R$. Pitschas Wandel (Fn. 3), 257 mwN. Überblick über die wissenschaftliche Begleitung der im folgenden geschilderten Entwicklung bei M. Stolleis Entwicklungsstufen der Verwaltungsrechtswissenschaft, in: Hoffmann-Riem/Schmidt-Aßmann/ Voßkuhle (Hrsg.) Grundlagen des Verwaltungsrechts, Band I, 2. Aufl. 2012, § 2, insb. Rn. 26 ff., 90 ff., 106 ff.

39 Siehe oben bei Fn. $23 \mathrm{ff}$.

${ }^{40}$ Insbesondere wurden bereits früh vormals private Unternehmen in Post, Eisenbahn und Elektrizität durch den Staat bzw. die Kommunen übernommen. Dieses formal-etatistische Regulierungsmodell bildete zugleich die Konkurrenzsituation der jungen Nationalstaaten ab. Siehe zum Post- und Telekommunikationswesen J. Masing Post und Telekommunikation, in: Isensee/Kirchhof (Hrsg.) Handbuch des Staatsrechts, Band 4, 3. Aufl. 2006, § 90 Rn. 2 ff.; E. Schilly in: Jeserich/Pohl/v. Unruh (Hrsg.) Deutsche Verwaltungsgeschichte, Band I, 1983, 448 (451 ff.) sowie ders. in: Jeserich/Pohl/ v. Unruh (Hrsg.) Deutsche Verwaltungsgeschichte, Band III, 1984, 385 ff. Zur Eisenbahn Stamm Eisenbahnverfassung (Fn. 27), 32 ff.; G. Hermes in: Ehlers/Fehling/Pünder (Hrsg.) Besonderes Verwaltungsrecht, Band I, 3. Aufl. 2012, § 25 Rn. 3 ff.; H. Seidenfus in: Jeserich/Pohl/v. Unruh (Hrsg.) Deutsche Verwaltungsgeschichte, Band III, 1984, 358 ff. Für die Energieversorgung unter Berücksichtigung des EnWG 1935 Hermes Infrastrukturverantwortung (Fn. 17), 288 ff. Zur Verstaatlichung des Fernstraßenwesens siehe R. Bartlsperger Das Fernstraßenwesen in seiner verfassungsrechtlichen Konstituierung, 2006, 85 ff. Neben den überregionalen Vernetzungen, die oftmals in die Hand der Gesamtstaaten gelangten, standen die örtlichen Infrastruktureinrichtungen wie etwa die Wasser- und Abwasserversorgung, vgl. J. Hellermann Örtliche Daseinsvorsorge und gemeindliche Selbstverwaltung, 2000, 16 ff.; zur kommunalen Gasversorgung siehe W. Hofmann in: Jeserich/Pohl/v. Unruh (Hrsg.) Deutsche Verwaltungsgeschichte, Band III, 1984, 578 ff.; weiterhin empirisch H.-D. Brunckhorst Kommunalisierung im 19. Jahrhundert, 1978, 25 ff. Zur Leistung des öffentlichen Sachenrechts für die Bewältigung dieser Aufgaben vgl. bereits $O$. Mayer Verwaltungsrecht, Band II, 3. Aufl. 1924, 72 ff., wonach die Einführung von Jedermannsrechten als wesentlicher Anschluss an das allgemeine rechtsstaatliche Verwaltungsrecht gelten kann. Gegen die Polemik vom rechtstatsächlich blinden rechtsstaatlichen Verwaltungsrecht (E. Forsthoff Verwaltungsrecht I, 10. Aufl. 1973, 52 ff., der das Anstaltsrecht verkürzend als ,große Lücke im System der rechtsstaatlichen Verwaltung" (53) kennzeichnet) überzeugend Jellinghaus (Fn. 26), 16 ff., mit umfassenden Nachweisen aus der zeitgenössischen Literatur. In den USA hat sich dagegen als Regelfall ein Modell des Zusammenwirken privilegierter Privatunternehmen mit staatlichen Behörden, die Monopol-Konzessionen erteilten, Zuschüsse 
einer kurzen, europarechtlich flankierten Privatisierungseuphorie ${ }^{42}$ sind seit einiger Zeit hybride Konzepte der Regulierung ${ }^{43}$ in den Vordergrund

zahlten und Leistungen abnahmen, immer weiter verfeinert, das dann für das gegenwärtig auch in Europa verfolgte Regulierungskonzept ieS (s. unten bei Fn. 43 f.) Pate stand, vgl. J. Masing AöR 128 (2003), 558 (568 ff.); O. Lepsius Regulierungsrecht in den USA: Vorläufer und Modell, in: Fehling/Ruffert (Hrsg.) Regulierungsrecht, 2010, § 1 Rn. 11-34.

${ }^{41}$ Vgl. bereits Fn. 33. Zur allmählichen Verortung des Planungsgedankens im rechtsstaatlichen Gesamtgefüge des Grundgesetzes zunächst $M$. Imboden Der Plan als verwaltungsrechtliches Institut, VVDStRL 18 (1960), 113 ff.; E. Forsthoff in: Kaiser (Hrsg.) Planung III, 1968, 21 (22 ff.); Th. Ellwein Politik und Planung, 1968, 58 f.; insb. für die Raumordnung P. Oberndorfer DV 5 (1972), 257 (258); F. Ossenbühl Welche normativen Anforderungen stellt der Verfassungsgrundsatz des demokratischen Rechtsstaats an die planende staatliche Tätigkeit, dargestellt am Beispiel der Entwicklungsplanung, Gutachten B zum 50. Deutschen Juristentag, 1974, insb. B 150 ff., 160 ff.; E. SchmidtAßmann DÖV 1974, 541 (542 ff.); W. Hoppe DVBl. 1974, 641 ff.; E.-W. Böckenförde Der Staat 11 (1972), 429 (429 ff.).

42 Aktueller Überblick über das Spektrum bei $A$. Voßkuhle Neue Verwaltungsrechtswissenschaft, in: Hoffmann-Riem/Schmidt-Aßmann/Voßkuhle (Hrsg.) Grundlagen des Verwaltungsrechts, Band I, 2. Aufl. 2012, § 1 Rn. 58 ff. Zum Leitmodell der materiellen Privatisierung insbesondere im kommunalen Bereich der Versorgung P. Badura FS Starck, 2007, 153 (158 ff.); als Beispiel für den Bereich des ÖPNV M. Fehling/K. Niehus DÖV 2008, 662 ff. Zur Privatisierung als juristischer Herausforderung M. Burgi Funktionale Privatisierung, 1999, 1 ff., vgl. zu den verfassungsrechtlichen Vorgaben 175 ff.; s.a. J. A. Kämmerer Privatisierung, 2001, 426 ff.; Ch. Gramm Privatisierung und notwendige Staatsaufgaben, 2001, 340 ff.; W. Weiss Privatisierung und Staatsaufgaben, 2002, 125. Vgl. zum Konzept des public-private-partnership für den Straßenbereich E. Gawel IR 2011, 4 ff., 29 ff.; S. Weisheit Privatisierung von Bundesautobahnen, 2011, insb. 129 ff.; zum Bereich der Städtebauförderung U. Battis/J. Kersten LKV 2006, 442 (mit Typologie 443 ff.); Überblick bei Th. Mann FS Püttner, 2006, 109 (113 ff.); umfassend F. Siebler Privilegierung von Public-Private-Partnerships im europäischen Vergaberecht, 2013, 67 ff. Kritische Bilanz zuletzt bei M. Hochhuth (Hrsg.) Rückzug des Staates und Freiheit des Einzelnen - Die Privatisierung existenzieller Infrastrukturen, 2013. Affirmativ dagegen G. Ambrosius ZöG 34 (2011), 214 (215 f.).

${ }^{43}$ Grundlegend für die Netzstrukturen Hermes Infrastrukturverantwortung (Fn. 17), 356 ff.; M. Ruffert AöR 124 (1999), 327 ff. Konzeptionell dann insb. J. Masing Die Verwaltung 36 (2003), 1 ff.; M. Fehling Regulierung als Staatsaufgabe im Gewährleistungsstaat Deutschland, in: Hill (Hrsg.) Die Zukunft des öffentlichen Sektors, 2006, 91 (92 ff.); zur Einordnung in die Wirtschaftsverfassung O. Lepsius Verfassungsrechtlicher Rahmen der Regulierung, in: Fehling/Ruffert (Hrsg.) Regulierungsrecht, 2010, § 4 Rn. 1 ff.; zu den ,versorgungssichernden Instrumenten“ der Regulierung $M$. Fehling Instrumente und Verfahren, ebd., § 20 Rn. 44 ff. Zum Begriff der „Infrastrukturregulierung" W. Hoffmann-Riem Rechtsformen, Handlungsformen, Bewirkungsformen, in: Hoffmann-Riem/Schmidt-Aßmann/Voßkuhle (Hrsg.) Grundlagen des Verwaltungsrechts, Band II, 2. Aufl. 2012, § 33 Rn. 94 ff. Zur Entwicklung jeweils mit Überblick über die europarechtliche Rechtslage zuletzt die Beiträge in: Ruffert (Hrsg.) Europäisches sektorales Wirtschaftsrecht (EnzEur Band 5), 2013: J. Kühling Telekom- 
getreten, die ein neues Bild des Gewährleistungsstaats etabliert haben. ${ }^{44}$ Vor allem ist aber zu betonen, dass bestimmte Sektoren sich der jeweiligen Gesamttendenz widersetzten ${ }^{45}$ und immer wieder auch Gegenentwicklungen $\mathrm{zu}$ beobachten waren, wie zuletzt etwa unter dem Stichwort der „Rekommunalisierung“. 46

Im Ergebnis ist bis in die Gegenwart eine vielgestaltige Rechtslage vorzufinden: Unter Bezeichnungen wie Energierecht, Straßenrecht, Eisenbahnrecht, Telekommunikationsrecht usw. sind in unterschiedlichen Kombinationen Anteile des „klassischen“ privatnützigen Genehmigungsrechts, ${ }^{47}$ des spezifischen Ordnungsrechts, ${ }^{48}$ des Rechts der öffentlichen

munikationsrecht, $§ 4$ Rn. 14 ff.; M. Ludwigs Energierecht, $§ 5$ Rn. 36 ff., 260 ff.; für das Eisenbahnrecht $M$. Knauff Transportrecht, $\S 6$ Rn. 120 ff. Stellvertretend zur Kritik des Modells K. F. Gärditz AöR 135 (2010), 251 ff.

${ }^{44}$ Grundlegend E. Schmidt-Aßmann in: Hoffmann-Riem/Schmidt-Aßmann/Schuppert (Hrsg.) Reform des Allgemeinen Verwaltungsrechts. Grundfragen, 1993, 11 (43 f.); W. Hoffmann-Riem Tendenzen in der Verwaltungsrechtsentwicklung, DÖV 1997, 433 (441 f.); M. Eifert Grundversorgung mit Telekommunikationsleistungen im Gewährleistungsstaat, 1998; G.F. Schuppert Verwaltungswissenschaft, 2000, 404 ff., 898 ff.; Voßkuhle (Fn. 3), 285 ff., 310 ff. Zur Kanonisierung Franzius Gewährleistung (Fn. 3), insb. 549 ff., 630 ff.; zur kritischen Reflexion etwa $R$. Wahl Herausforderungen und Antworten: Das Öffentliche Recht der letzten fünf Jahrzehnte, 2006, 83 ff.; K.-H. Ladeur Der Staat 48 (2009), 163 ff. Zum Zusammenhang von Regulierung und Gewährleistungskonzept H. Wißmann Art. Regulierung, Ev. Staatslexikon, 2006, Sp. 1978 (1981 ff.).

${ }_{45}$ Prototypisch zum Recht der Wasserversorgung J. Masing VerwArchiv 95 (2004), 151 (160 ff.); A. Glöckner Kommunale Infrastrukturverantwortung und Konzessionsmodelle, 2009, 165 ff.; J. Brehme Privatisierung und Regulierung der öffentlichen Wasserversorgung, 2010, 154 ff.; J. Kühling DVB1. 2010, 205 ff.; M. HellriegellTh. Schmitt IR 2011, 294 ff.; vgl. zum aktuellen Stand unten Fn. 119.

${ }^{46}$ Für den historischen Gesamtkontext $P$. Collin JZ 2011, 274 ff. Zum Stichwort der „Rekommunalisierung“ H. Bauer DÖV 2012, 329 ff. Zum Beispiel der Berliner Wasserbetriebe mit Rückblick auf die zugrundeliegende Privatisierungskonzeption K. Lederer IR 2012, 302 ff.; zuletzt zum Rückkauf der Leitungsnetze durch die Freie und Hansestadt Hamburg nach Volksentscheid FAZ vom 20.9.2013, 14. Zur Kategorie des schlichten Verwaltungshandelns in Bezug auf nach wie vor öffentlich-rechtlich erbrachte Infrastrukturdienste G. Hermes Schlichtes Verwaltungshandeln, in: Hoffmann-Riem/ Schmidt-Aßmann/Voßkuhle (Hrsg.) Grundlagen des Verwaltungsrechts, Band II, 2. Aufl. 2012, $\S 39$ Rn. 44 ff. Fragend zum „Gewährleistungsstaat in der Krise“ bereits M. Knauff DÖV 2009, $581 \mathrm{ff}$.

$47 \mathrm{Zu}$ dessen Funktion und Ausdifferenzierung J. Masing Der Rechtsstatus des Einzelnen im Verwaltungsrecht, in: Hoffmann-Riem/Schmidt-Aßmann/Voßkuhle (Hrsg.) Grundlagen des Verwaltungsrechts, Band I, 2. Aufl. 2012, § 7 Rn. 165 ff.

${ }^{48}$ Zur Rolle des Ordnungsrechts im Öffentlichen Wirtschaftsrecht D. Ehlers in: ders./Fehling/Pünder (Hrsg.) Besonderes Verwaltungsrecht, Band I, 3. Aufl. 2012, § 1 Rn. 22; Vgl. auch J. Ziekow Öffentliches Wirtschaftsrecht, 2. Aufl. 2010, § 10 Rn. 28; a.a.O. $\S 3$ Rn. 3; konzeptionell P. M. Huber Überwachung, in: Hoffmann-Riem/ 
Sachen und des Anstaltsrechts, ${ }^{49}$ des kommunalen Wirtschaftsrechts, ${ }^{50}$ des Planungsrechts, ${ }^{51}$ des Vergabe- und Kartellrechts ${ }^{52}$ sowie des Regulie-

Schmidt-Aßmann/Voßkuhle (Hrsg.) Grundlagen des Verwaltungsrechts, Band III, 2. Aufl. 2013, $\S 45$, insb. Rn. 7 f., 28 f.; grundlegend $R$. Gröschner Überwachungsrechtsverhältnis, 1992, insb. $119 \mathrm{ff}$.

${ }^{49}$ Siehe bereits oben Fn. 40. Aktuelle Gesamtdarstellungen bei H. J. Wolffl O. BachoflR. Stober Verwaltungsrecht II, 7. Aufl. 2010, $\$ 74$ ff.; H.-J. Papier in: Erichsen/Ehlers (Hrsg.) Allgemeines Verwaltungsrecht, 14. Aufl. 2010, §§ 38 ff. Zu den Schwierigkeiten, „die Identität“ des seinerseits vielgestaltigen Rechts der öffentlichen Sachen ,als Rechtsgebiet“ zu bestimmen, vor allem P. Axer Die Widmung als Schlüsselbegriff des Rechts der öffentlichen Sachen, 1992, insb. 23 ff., 218 ff.; sowie jüngst am Beispiel öffentlicher Friedhöfe ders. DÖV 2013, 165 ff. Von einem weiten, u.a. das Anstaltsrecht sowie verschiedene planungsrechtliche Aspekte berührenden Begriff des öffentlichen Sachenrechts gehen etwa auch die Berichte von F.-J. Peine JZ 1996, 350 ff., 398 ff.; sowie dems. JZ 2006, 593 ff., aus. Überblick über aktuelle Fragen des straßenrechtlichen Nutzungsregimes bei $T$. Siegel NVwZ 2013, $479 \mathrm{ff}$.

${ }^{50}$ Vgl. für den einschlägigen Gesamtüberblick D. Ehlers DVBl. 1998, 497 ff.; M. Ruffert VerwArch 92 (2001), 27 ff.; A. Katz Kommunale Wirtschaft, 2004; zuletzt J. Peters Dogmatik der Kommunalwirtschaft, 2012; zu kommunalen Unternehmen J. Suerbaum in: Ehlers/Fehling/Pünder (Hrsg.) Besonderes Verwaltungsrecht, Band I, 3. Aufl. 2012, $\S 13$; umfassend W. Hoppe/M. Uechtritz/H.-J. Reck (Hrsg.) Handbuch Kommunale Unternehmen, 3. Aufl. 2012. Zur Beziehung zwischen kommunaler Wirtschaftstätigkeit und Daseinsvorsorge vor allem J. Hellermann Örtliche Daseinsvorsorge und gemeindliche Selbstverwaltung, 2000; K. Rennert Die Verwaltung 35 (2002), 319 ff.; G. Britz Die Verwaltung 37 (2004), $145 \mathrm{ff}$.

${ }^{51}$ Siehe bereits oben bei Fn. 33, 41. W. Köck Planung als eigenständige Handlungsform, in: Hoffmann-Riem/Schmidt-Aßmann/Voßkuhle (Hrsg.) Grundlagen des Verwaltungsrechts, Band II, 2. Aufl. 2012, § 37, insb. Rn. 20 f.; Wahl Herausforderungen (Fn. 44), 47 ff.; M. Beckmann in: Erbguth/Kluth (Hrsg.) Planungsrecht in der gerichtlichen Kontrolle, 2011, 123 ff. Das Planungsrecht spielt als zentrale Handlungsplattform für die Entscheidung über Infrastrukturen eine entscheidende Rolle, siehe dazu weiter III. 1., IV. 2.

${ }^{52}$ Insbesondere M. Bungenberg Vergaberecht im Wettbewerb der Systeme, 2007, $133 \mathrm{ff}$. Allgemeiner Überblick zur Situation des Vergaberechts bei S. Hertwig Praxis der öffentlichen Auftragsvergabe, 4. Aufl. 2009; J. Ziekow Öffentliches Wirtschaftsrecht, 2. Aufl. 2010, § 9; S. Storr in: Ruthig/ders., Öffentliches Wirtschaftsrecht, 3. Aufl. 2011, $\S 10 ;$ H. Pünder in: Ehlers/Fehling/ders. (Hrsg.) Besonderes Verwaltungsrecht, Band I, 3. Aufl. 2012, § 17, dort Rn. 17 zur Bindung Privater im Sektorenvergaberecht nach $\S 98$ Nr. 4 GWB; zur Europäisierung bereits $O$. Dörr JZ 2004, 703 ff.; M. Bungenberg/ P. M. Huber/R. Streinz (Hrsg.) Daseinsvorsorge und öffentliches Beschaffungswesen nach dem Lissabonner Vertrag, 2011; zuletzt A. Gyulai-Schmidt VergabeR 2012, $809 \mathrm{ff}$. Siehe weiter M. Fehling in: Pünder/Schellenberg (Hrsg.) Vergaberecht, 2011, § 97 Rn. 7 ff.; siehe auch J. Ziekow in: ders./Völlink (Hrsg.) Vergaberecht, 2011, 1 ff.; A. Schwab in: Heuvels/Höß/Kuß/Wagner (Hrsg.) Vergaberecht, 2013, 1 ff. 
rungsrechts $^{53} \mathrm{zu}$ jeweils eigengearteten Infrastrukturteilrechtsordnungen verbunden. ${ }^{54}$

Vom frühen Enteignungsrecht bis zum europäischen Regulierungsverwaltungsrecht der Gegenwart lässt sich so eine erste Grundlinie nachzeichnen: Durchgesetzt hat sich die Einsicht, dass die Frage des Umfangs, der Qualität und des Zugangs zu Infrastrukturen allgemeinverbindlich geklärt werden muss, weil hier über Vorbedingungen von Freiheit entschieden wird, neben denen individuelle Entscheidungen der Eingriffs- und Leistungsverwaltung geradezu als Randfunktion erscheinen. ${ }^{55}$ Bei der konkreten Entscheidung über öffentliche Infrastrukturen und ihre Durchführung wirken private Interessen und Gegeninteressen und die Vertreter der Allgemeinheit dann regelmäßig zusammen. ${ }^{56}$ Die Festlegung auf bestimmte Akteure, sei es im etatistischen Ansatz der Daseinsvorsorge, ${ }^{57}$ sei

$53 \mathrm{Vgl}$. bereits oben bei Fn. 43. Im Zusammenhang J. Masing Soll das Recht der Regulierungsverwaltung übergreifend geregelt werden, Gutachten D zum 66. DJT, 2006, insb. D 18 ff. Vergleichsweise klar ist zu erkennen, dass die Vorstellung der Regulierung als zeitlich begrenztem Privatisierungsfolgenrecht, das letztlich im Kartellrecht aufgehen könne, die Eigenart der regulierten Strukturen verkennt. Für den Übergang von einem sektoral begrenzten zu einem allgemeinen Regelungskonzept vgl. die Breite der Beiträge in Fehling/Ruffert (Hrsg.) Regulierungsrecht, 2010.

54 Vgl. zur Vielfalt der Regelungsmodelle bereits R. Schmidt Der Staat 42 (2003), 225 (242 ff.). Zum Konzept der ,,säulenübergreifenden“ Sektoralität M. Ruffert Sektorales Wirtschaftsrecht als Teil des europäischen Wirtschaftsrechts, in: ders. (Hrsg.) Europäisches sektorales Wirtschaftsrecht (EnzEur Band 5), 2013, § 1 Rn. 9 ff., 37 f.; für die sektorenübergreifende Rückbindung J. Kühling Sektorenspezifische Regulierung, 2004, 444 ff. Zur verwaltungsrechtswissenschaftlichen Analyse der unterschiedlichen Regulierungsstrategien typisierend $M$. Eifert Regulierungsstrategien, in: HoffmannRiem/Schmidt-Aßmann/Voßkuhle (Hrsg.) Grundlagen des Verwaltungsrechts, Band I, 2. Aufl. 2012, § 19 Rn. 23 ff.

55 Diese zentrale Einsicht bei H. Faber Verwaltungsrecht, 4. Aufl. 1996, 170. Beispielhaft lässt sich dieser Zusammenhang zwischen der Ausgestaltung der Infrastruktur des Internets und den Kommunikationsfreiheiten zeigen, vgl. zur notwendigen Leistungsfähigkeit $H$. Greve KuR 2013, 87 ff.; zum grundrechtlich gebotenen Datenschutz andererseits G. Schulz DUD 2012, 395 ff.; vgl. auch J. Masing FS Wahl, 2011, 147 (156 ff.); B. Holznagel Erosion demokratischer Öffentlichkeit?, VVDStRL 68 (2009), 381 (391 ff.). Zu den verschiedenen Perspektiven auf die staatliche Infrastrukturverantwortung M. Cornils AöR 131 (2006), 378 (384 ff.). Zum globalen Maßstab D. Acemoglu/J. A. Robinson Why Nations fail, 2013, $70 \mathrm{ff}$., $368 \mathrm{ff}$.

56 Zum Konzept des Gewährleistungsrechts bereits Fn. 44. Vgl. zu den vielfältigen Erscheinungsformen der Erfüllung öffentlicher Aufgaben durch Private den aktuellen Überblick bei $H$. Schulze-Fielitz Grundmodi der Aufgabenwahrnehmung, in: Hoffmann-Riem/Schmidt-Aßmann/Voßkuhle (Hrsg.) Grundlagen des Verwaltungsrechts, Band I, 2. Aufl. 2012, § 12 Rn. 91 ff. Vgl. weiter unten bei Fn. 60.

57 Vgl. kennzeichnend für die totalitäre Perspektive auf die staatliche Massenverwaltung jenseits subjektiver Rechte E. Forsthoff Die Verwaltung als Leistungsträger, 1938, 
es in der ideologischen Überhöhung vollständiger Privatisierung, ${ }^{58}$ ist demgegenüber Ausnahme geblieben. Deshalb ist die Mischung von Regulierungsansätzen, sowohl zwischen unterschiedlichen Infrastrukturbereichen wie auch innerhalb eines Sektors, der typische Befund in Geschichte und Gegenwart. Das Infrastrukturrecht steht so - gegen die Bestrebung abschließender Kodifikation und Vertypung - in besonderer Weise für den beweglichen, reaktiven Charakter des Verwaltungsrechts. ${ }^{59}$

Eine solche Beobachtung weist freilich auf Folgefragen: Handelt es sich um eine bloße Unfertigkeit, die sich nicht aus der Sache, sondern aus der unterschiedlichen Abwehrkraft der betroffenen Fachbruderschaften gegenüber neuen Einsichten speist, also mit wissenschaftlichem Optimismus als temporäres Problem zu beurteilen ist? Oder wirken die Ordnungsideen des Allgemeinen Verwaltungsrechts im Infrastrukturrecht in dialektischer Wendung gerade dadurch, dass Alternativen bereitgestellt werden und Verschiedenheit so nicht nur ertragen, sondern als Gestaltungsinstru-

19 ff., insb. 21. Zur Spannung zwischen Fortführung der Begrifflichkeit und gewandelter Konzeption unter dem Grundgesetz Jellinghaus (Fn. 26), 15 f. Vgl. zur Debatte H. P. Bull Der Staat 47 (2008), 1 (2 ff.); M. Ronellenfitsch, in: Blümel (Hrsg.) Kolloquium aus Anlass des 100. Geburtstags von Prof. Dr. Dr. h.c. E. Forsthoff, 2003, 53 (63 ff.); kämpferisch ders. DVBl. 2008, 201 (201 mit Fn. 2); differenzierend J. Kersten/ S. - C. Lenski Die Verwaltung 42 (2009), 501 (505 f.); R. Ringwald Daseinsvorsorge als Rechtsbegriff, 2008, 71 ff.; insbesondere zur europarechtlichen Nutzung M. Möstl FS Badura 2004, 951 ff.; zum Rechtsvergleich mit dem service public Pielow Grundstrukturen (Fn. 16), 392 ff. Insgesamt kritisch R. Schmidt Der Staat 42 (2003), 225 (247); H. Butzer Sicherstellungsauftrag, in: Isensee/Kirchhof (Hrsg.) Handbuch des Staatsrechts, Band 4, 3. Aufl. 2006, § 74 Rn. 7 mit Fußnote 18; M. Krajewski VerwArchiv 99 (2008), 174 (178 ff.); O. Lepsius Verfassungsrechtlicher Rahmen der Regulierung, in: Fehling/Ruffert (Hrsg.) Regulierungsrecht, 2010, § 4 Rn. 86 ff.; stellvertretend für die weitere Nutzung des Begriffs zuletzt W. G. Leisner WiVerw 2011/2, 55 (65 f.); St. Schmahl WiVerw 2011/2, 96 ff.

58 Einschlägig etwa W. Möschel JZ 1988, 885 ff.; ders. FS Gernhuber, 1993, 905 ff.; Ch. Koenig FS Isensee, 2007, 375 ff.; konzeptionelle Abgrenzung bei U. Di Fabio JZ 1999, 585 ff. Zu den Wellenbewegungen politischer Strömungen und Moden hinsichtlich der Privatisierung mwN J. A. Kämmerer in: Ehlers/Fehling/Pünder (Hrsg.) Besonderes Verwaltungsrecht, Band 1, 3. Aufl. 2012, § 14 Rn. 32 ff.

${ }_{59}$ Gesamtüberblick über die Entwicklung bei $R$. Wahl Herausforderungen (Fn. 44), 76 ff. Anders gewendet spiegelt sich hier die Spannung zwischen „öffentlichen“ und „staatlichen“ Aufgaben, vgl. dazu breit Pielow Grundstrukturen (Fn. 16), 302 ff. Zur Kodifikationsidee einschlägig W. Kahl in: Hoffmann-Riem/Schmidt-Aßmann (Hrsg.) Verwaltungsverfahren und Verwaltungsverfahrensgesetz, 2002, 67 ff. (insb. 89 ff.); ders. Die Europäisierung des Verwaltungsrechts als Herausforderung an Systembildung und Kodifikationsidee, Die Verwaltung Beiheft 10 (2010), 39 (55 ff.); vgl. weiter H. SchulzeFielitz FS Schmidt-Aßmann, 2008, 135 ff. Zur Mischung von Regulierungsansätzen M. Burgi Funktionale Privatisierung, 1999, insb. 100 ff., 145 ff. 
ment wahrgenommen werden kann? Dafür ist nach Gemeinsamkeiten und Unterschieden zu fragen, die sich in den typischen Phasen der Planung, Bereitstellung und Pflege von Infrastrukturen ergeben.

\section{Der Verbund von Phasen im Infrastrukturrecht}

\section{Planung: Konzeption und Entscheidung}

\section{a) Infrastrukturinitiative - Infrastrukturschaffungspflichten \\ aa) Ausgangslage und Bestand rechtlicher Regelungen}

$\mathrm{Ob}$ öffentliche Infrastrukturen geschaffen werden, wird in der Regel in einem komplexen Prozess konkret-politisch entschieden. ${ }^{60}$ Die Initiative zu einer solchen Entscheidung kann von verschiedenen Akteuren ausgehen: Neben gesamtstaatlichen bzw. gemeineuropäischen Strukturvorgaben, die parlamentarisch und gouvernemental vereinbart werden, agieren - oft damit vernetzt ${ }^{61}$ - privatwirtschaftliche Konzerne, aber auch Vertreter der Bürgergesellschaft oder auch speziell eingesetzte sachverständige Gremien. ${ }^{62}$ Gleiches wiederholt sich auf den verschiedenen Ebenen bis in den lokalen Bereich. ${ }^{63}$

Dabei bestehen typischerweise keine ausdrücklichen verfassungsrechtlichen Vorgaben, „ob“ öffentliche Infrastrukturen $\mathrm{zu}$ schaffen sind. Die bekanntesten Ausnahmen von dieser Regel sind Art. 87e und Art. 87f GG, durch die den Bund die Verpflichtung trifft, bei Bahn, Post und Tele-

\footnotetext{
${ }^{60}$ Vgl. für eine Teilung in vier Unterphasen K. Waechter Großvorhaben (Fn. 4), $501 \mathrm{f}$.

${ }^{61}$ Aktuelle Diagnose zum Modell des Public-Private-Partnership J. Ziekow in: ders. (Hrsg.) Wandel der Staatlichkeit und wieder zurück?, 2011, 43 ff.; siehe auch H.J. Wolffl O. Bachof/R. Stober/W. Kluth, Verwaltungsrecht II, 7. Aufl. 2010, §93. Rechtsvergleichend M. Kment VerwArchiv 103 (2012), 63 (mit Typenbildung 67 ff.).

${ }^{62}$ Zur sogenannten „Endlagerkommission“ $\$ \S 3$ ff. Standortauswahlgesetz (StandAG) vom 23.7.2013, BGBl. I 2553.; ob damit allerdings eine Neutralität der entsprechenden Grundentscheidungen verbunden ist, bleibt zweifelhaft. Zur Neutralität im Planfeststellungsverfahren $K$. Waechter Großvorhaben (Fn. 4), 503 ff., 508 ff., 511 ff.; zur Einbeziehung von Sachverstand umfassend A. Voßkuhle HbdStR III, 3. Aufl. 2005, $\S 43$, insb. Rn. 58 ff., der auf das Erfordernis politisch-demokratischer Legitimation hinweist.

${ }^{63}$ Öffentliche Infrastrukturen und die Ansiedlung von Industrie oder auch neuer Bevölkerung sind dabei über wechselseitige Anreizsysteme eng und in empfindlicher Weise miteinander verbunden, so dass sich die ursprüngliche Initiative oftmals nicht klar ermitteln lässt, sondern sich die Akteursbeziehungen prozesshaft entwickeln. Vgl. weiter bereits bei Fn. $32 \mathrm{ff}$.
} 
kommunikation „flächendeckend angemessene und ausreichende Dienstleistungen“ zu gewährleisten. ${ }^{64}$ Hinzuweisen ist weiter darauf, dass das Landesverfassungsrecht aus konkreten historischen Gründen umfängliche Festlegungen zur Einrichtung und zum Betrieb öffentlicher Schulen enthält. ${ }^{65}$

Abgesehen von solchen Sonderlagen übernimmt das einfache Gesetz die Aufgabe, Infrastrukturschaffungspflichten $\mathrm{zu}$ kreieren. Inhaltlich kann es um den Gesamtausbau wie um den Zugang des Einzelnen gehen, z.B. im Bereich Straßenbau, der Wasser- und Abwasserversorgung, Einrichtungen des Gesundheits- und des Bildungswesens. ${ }^{66}$ Von solchen Vorgaben sind in gewährleistungsrechtlich regulierten Bereichen auch private Unternehmen betroffen, etwa in den Bereichen Telekommunikation,

${ }^{64}$ Art. 87f Abs. 1 GG; gleichsinnig Art. 87e Abs. 4 GG. Vgl. BVerfGE 108, 169 (182 f.) - Telekommunikationsgesetz. Zu verstehen ist diese Gewährleistungsverantwortung als politischer Großkompromiss nur als Ausgleichsgröße der Umstellung auf eine privatwirtschaftliche Erfüllung dieser Aufgaben, vgl. Art. 87e Abs. 3, Art. 87f Abs. 2 1 GG. Im europäischen Primärecht finden sich dagegen mit den Bestimmungen zu den transeuropäischen Netzen und zur Energiepolitik nur vorerst offene Zielbestimmungen, vgl. Art. 170 f. AEUV, vgl. dazu Ch. Calliess in: ders./Ruffert (Hrsg.) EUV/AEUV, 4. Aufl. 2011, Art. 170, insb. Rn. 3, sowie Art. 194 Abs. 2 UA 2 AEUV, dazu knapp Ch. Calliess in: ders./Ruffert (Hrsg.) EUV/AEUV, 4. Aufl. 2011, Art. 194 Rn. 28; M. Ludwigs Energierecht, in: Ruffert (Hrsg.) Europäisches sektorales Wirtschaftsrecht (EnzEur Band 5), 2013, § 5 Rn. 56 ff.; vgl. auch J. Gundel Europäisches Energieverwaltungsrecht, in: Terhechte (Hrsg.) Verwaltungsrecht der Europäischen Union, 2011, § 23 Rn. 14 ff. Die knappe Benennung von Infrastrukturfragen im AEUV wird durch eine Masse sekundärrechtlicher Vorgaben überlagert; ungeachtet ihres Anwendungsvorrangs sind diese funktional jedoch nicht als verfassungsrechtliche Vorgaben anzusprechen, weil sie als konkrete politische Einigung zustandekommen.

${ }^{65}$ H. Wißmann Verfassungsrechtliche Vorgaben der Verwaltungsorganisation, in: Hoffmann-Riem/Schmidt-Aßmann/Voßkuhle (Hrsg.) Grundlagen des Verwaltungsrechts, Band I, 2. Aufl. 2012, § 15 Rn. 12 mit Fn. 59. Zur Genese ders. Homeschooling (Fn. 22), 26 ff.

${ }^{66}$ Vgl. zur vom Bundesrechnungshof betriebenen Debatte um den Umfang der Bundesfernstraßen M. Sauthoff DÖV 2009, 974 ff.; B. Witting DVBl. 2010, 408 ff. Parallel zB $\S \S 1$ ff. LStrAusbauG NRW (Bedarfsplan per Gesetz, dann Ausbauplan); zur Rolle privater Akteure bei Fn. 106 ff. Pflicht zur Sicherstellung der öffentlichen Wasserversorgung durch Gemeinden zB nach § 47a LWG NRW auf Grundlage des Wasserversorgungsberichts, § 50a LWG NRW; Abwasserableitung durch Gemeinden, § 53 Abs. 1 LWG NRW. Für Krankenhäuser in NRW $\S 12$ ff. KHGG NRW (ministerieller Krankenhausplan, Rahmenvorgaben und regionale Planungskonzepte). Für eine Infrastrukturpflicht ohne (notwendige) physische Einrichtung in den Rettungsdienstgesetzen vgl. die Verpflichtung der Kreise und kreisfreien Städte zur Sicherstellung einer bedarfsgerechten Versorgung, zB $\S 6$ RettungsdienstG NRW, Grundlage bildet ein Bedarfsplan, $\S 12$. Allgemeine Übersicht für die kommunalen Pflichten bei Glöckner Infrastrukturverantwortung (Fn. 45), $71 \mathrm{ff}$. 
Eisenbahn und Energie, hier in der Regel angeleitet durch europäisches Sekundärrecht. ${ }^{67}$ Die zum Einsatz kommenden Instrumente, die von prozedural entwickelten Szenarioplanungen und Netzentwicklungsplänen bis zu gesetzlich beschlossenen Bedarfsplänen reichen, spiegeln auch insoweit die unterschiedlichen Verläufe und Grundbedingungen der Sektoren wider. ${ }^{68}$ Eine wichtige Rolle spielen selbstverständlich neben den gesetz-

${ }^{67}$ Im Regelungsbereich des $T K G$ ist auf die Realisierung der Anschlussverpflichtung als Teil der Universaldienstpflichten hinzuweisen, insb. § 78 Abs. 2 Nr. 1 iVm § 84 I TKG, vgl. M. Cornils in: Geppert/Schütz (Hrsg.) Beck'scher TKG-Kommentar, 4. Aufl. 2013, § 78 Rn. 32 ff.; zur europarechtlichen Lage J. Kühling Telekommunikationsrecht, in: Ruffert (Hrsg.) Europäisches sektorales Wirtschaftsrecht (EnzEur Band 5), 2013, § 4 Rn. 136 ff., insb. Rn. 139 zum dynamischen Begriff des ,funktionalen Internetzugangs“, für den allerdings ein nationaler Gestaltungsspielraum anerkannt ist. Insb. zur Frage des Breitbandausbaus proaktiv B. HolznagellS. Deckers DVBl. 2009, 482 (488 f.); dies/M. Schramm NVwZ 2010, 1059 ff., sowie kritisch J. Kühling/M. Biendl DÖV 2012, 409 ff. Zu den entsprechenden Duldungspflichten von Grundstückseigentümern bzw. der öffentlichen Hand M. Freund/T.-L. Bary NVwZ 2012, 1504 ff. („Hausstich“); U. Rathgeb NVwZ 2012, 270 ff. (Wegenutzung); vgl. umfassend zu den einschlägigen Neuregelungen des TKG 2012 B. KindlM. Schramm N\&R 2012, 140 ff. Zu den entsprechenden (Ausbau-)Pflichten nach dem EnWG und EEG in Bezug auf Übertragungsund Verteilernetze (aktuell: §§ 11, 12, 12a-12e, 17, 17a-17d, 18, 36 EnWG; §§ 5, 9 EEG; $\S 6$ NABEG) unter dem Stichwort der Versorgungssicherheit allgemein $K$. Bourwieg in: Britz/Hellermann/Hermes (Hrsg.) EnWG, 2. Aufl. 2010, § 11 Rn. 31 ff.; insb. zu den kommunalen Verteilernetzbetreibern K. Rauch IR 2008, 218 ff.; zur Beschleunigung des Netzausbaus nach EnWG und NABEG M. Appel UPR 2011, 406 ff.; vgl. M. Rodi IR 2012, 242 (246 f.); konzeptionell mit Vergleich zur Investionsplanung im Verkehrssektor J. P. Schneider Infrastrukturausbau als Aufgabe des Regulierungsrechts, in: Fehling/ Grewlich (Hrsg.) Struktur und Wandel des Verwaltungsrechts, 2011, 69 (75 ff.); kritisch zur Netzausbauverpflichtung unter Berücksichtigung der Elektrizitätsbinnenmarktrichtlinie A. Glaser DVBl. 2012, 1283 (1286 f.). Zum europarechtlichen Rahmen M. Ludwigs Energierecht, in: Ruffert (Hrsg.) Europäisches sektorales Wirtschaftsrecht (EnzEur Band 5), 2013, § 5 Rn. 36 ff. (Energieversorgungssicherheit als dritte Säule der europäischen Energiepolitik neben Binnenmarkt und Umweltschutz). Für die Entwicklung des Schienennetzes gilt das Bundesschienenwegeausbaugesetz und der damit verbundene Bedarfsplan (BGBl. I 2004, 2322 f.); zur Überprüfung nach $§ 4$ vgl. Bericht des Bundesministeriums für Verkehr, Bau und Stadtentwicklung vom 11.11.2010; zum Gesetzentwurf 2013 vgl. unten Fn. 121. Insgesamt sind Regulierungsverfügungen trotz gewisser struktureller Parallelität nicht klassische Planungsverfügungen, sondern pflichtengenerierende Ordnungsverfügungen, vgl. dazu Ch. Bumke in: Hoffmann-Riem/ Schmidt-Aßmann/Voßkuhle (Hrsg.) Grundlagen des Verwaltungsrechts, Band II, 2. Aufl. 2012, § 35 Rn. 102 mit Fn. 354. Zum Zusammenhang zwischen Eigentumsfreiheit und „Systemrelevanz“ M. Droege DVBl. 2009, 1415 (1419 ff.).

${ }^{68}$ Vgl. bereits Fn. 67. Zum Bundesverkehrswegeplan Th. Groß VerwArchiv 104 (2013), 1 (2. ff.). Aktuell zur Infrastrukturentwicklung durch Leitstrategie und Geschäftsplan im europäischen Eisenbahnrecht Ch. Lerche N\&R 2013, 27 (29 f.). Siehe 
lich angeordneten nach wie vor die insbesondere auf kommunaler Ebene freiwillig erbrachten Leistungen und ihre Infrastruktur. ${ }^{69}$

\section{bb) Keine allgemein-ungeschriebenen Infrastrukturschaffungspflichten}

Eine allgemeine, etwa grundrechtlich in der Berufsfreiheit wurzelnde Infrastrukturschaffungspflicht überall dort, wo der Wettbewerb solche Strukturen braucht und selber nicht bewältigen kann, ist dagegen abzulehnen. ${ }^{70}$ Weder das Grundgesetz noch das europäische Recht liefern den Staat in solcher Weise an den Markt aus. Eine andere Betrachtung verwechselte die Legitimationsebenen von Wettbewerbsfreiheit und politischer Gesamtordnung. ${ }^{71}$ Gleiches gilt dem Grunde nach auch für individuelle Versorgungsansprüche; nur ganz ausnahmsweise kann anderes für

W. Köck in: Hoffmann-Riem/Schmidt-Aßmann/Voßkuhle (Hrsg.) Grundlagen des Verwaltungsrechts, Band II, 2. Aufl. 2012, §37 Rn. 87 ff. am Beispiel des Krankenhausplans. Neben den Netzausbau kann auch das Netzmanagement treten, vgl. dazu M. Martini VerwArchiv 102 (2011), 315 (317 f.). Vgl. auch Schmidt-Aßmann Ordnungsidee (Fn. 3), Rn. $6 / 98$ f.

${ }^{69}$ Vgl. dazu etwa $U$. Berlit NDV 2012, 419 ff., 479 ff., insb. unter dem Aspekt der Finanzierung der entsprechenden Infrastruktur. Zur kommunalen Aufgabenlehre siehe H. C. Röhl in: Schoch (Hrsg.) Besonderes Verwaltungsrecht, 15. Aufl. 2013, 9 Rn. 60 ff.; R. Stober Kommunalrecht in der Bundesrepublik Deutschland, 3. Aufl. 1996, 32 ff.; A. Gern Deutsches Kommunalrecht, 3. Aufl. 2003, Rn. 227 ff.; M.-E. Geis Kommunalrecht, 2. Aufl. 2011, 50 ff.; T. I. Schmidt Kommunalrecht, 2011, Rn. 226 ff.

${ }^{70}$ In diese Richtung Gramm Privatisierung (Fn. 42), 285 f., 321 ff. Entsprechende Argumente gehen in der Regel von volkswirtschaftlichen Modellen aus, dass bestimmte Infrastrukturen im vernünftigen Wettbewerb nicht bereitgestellt werden, und schließen daraus, dass hier dann eine staatliche Pflicht eintritt; dagegen bereits $M$. Knauff Der Gewährleistungsstaat: Reform der Daseinsvorsorge, 2004, 273; Franzius Gewährleistung (Fn. 3), 390 f. mit Fn. 125. Zum folgenden mwN H. Wißmann Verfassungsrechtliche Vorgaben der Verwaltungsorganisation, in: Hoffmann-Riem/Schmidt-Aßmann/Voßkuhle (Hrsg.) Grundlagen des Verwaltungsrechts, Band I, 2. Aufl. 2012, § 15 Rn. 10 ff. Vgl. rechtsvergleichend Pielow Grundstrukturen (Fn. 16), 115 ff., $288 \mathrm{ff}$.

${ }^{71}$ Die staatliche „Funktionsgewährleistungspflicht“ des Art. 14 AEUV bezieht sich deshalb nur auf zuvor definierte Dienste von allgemeinem öffentlichen Interesse, vgl. Ch. Jung in: Calliess/Ruffert (Hrsg.) EUV/AEUV, 4. Aufl. 2011, Art. 14 Rn. 22 in Verbindung mit 9, 12 f.; Zum Gestaltungsspielraum der Nationalstaaten vgl. auch EuG, Rs. T-289/03, Slg. 2008, II-00 081,00081 Rn. 166-169, 172 (BUPA/Kommission); Europäische Kommission, Leistungen der Daseinsvorsorge in Europa, 20.9.2000, KOM (2000) 580 endg., Rn. 22. Auch die Abgrenzung von Tätigkeitsfeldern zwischen Europäischer Union und Nationalstaaten in BVerfGE 123, 267 (369 ff., 381 ff., 406 ff.) Lissabon-Vertrag, kann unter dem Aspekt der politischen Entscheidbarkeit über bestimmte Dienstleistungen rekonstruiert werden. Umgekehrt kann auch eine Begrenzung öffentlicher Infrastrukturen nicht aus dem Marktprinzip folgen. 
existentielle Güter dargelegt werden. ${ }^{72}$ Es wäre eine Fehldeutung, in jedem Fall eine Überforderung der Konzeption staatlicher Gewährleistungsoder Auffangverantwortung ${ }^{73}$ (ja der Verfassung überhaupt), wenn mit ihr eine materielle Staatsaufgabenlehre verbunden würde: Richtigerweise setzt sie den andernorts bestimmten Umfang staatlicher Aufgaben bereits voraus. ${ }^{74}$ Gleiches gilt auch für die Kompetenzordnung, insbesondere für

72 Vgl. BVerfGE 66, 248 (258) - EnWG-Enteignung; 82, 60 (79 f.) - Steuerfreies Existenzminimum; 125, 175 (222 ff.) - Regelleistung. Überblick mwN bei M. Ruffert Vorrang der Verfassung und Eigenständigkeit des Privatrechts, 2001, 256 ff.; H. Wißmann Verfassungsrechtliche Vorgaben der Verwaltungsorganisation, in: HoffmannRiem/Schmidt-Aßmann/Voßkuhle (Hrsg.) Grundlagen des Verwaltungsrechts, Band I, 2. Aufl. 2012, $§ 15$ Rn. 12a ff. Im Einzelnen zu Ableitungen aus Sozialstaatsprinzip und Grundrechten in Bezug auf (kommunale) Infrastrukturschaffungspflichten F. BrosiusGersdorf VerwArchiv 98 (2007), 317 (328 ff.); vgl. in Bezug auf Lebensmittelversorgung zweifelhaft H. Butzer Sicherstellungsauftrag, in: Isensee/Kirchhof (Hrsg.) Handbuch des Staatsrechts, Band 4, 3. Aufl. 2006, § 74 Rn. 24; sehr weitgehender Ansatz bei W. Weiss Privatisierung und Staatsaufgaben, 2002, 149 ff.; skeptisch J. Hellermann Schutz der Verbraucher durch Regulierungsrecht, VVDStRL 70 (2011), 366 (375 f.). Umfassend W. G. Leisner Existenzsicherung im Öffentlichen Recht, 2007, 98 ff. Zur begrenzten Rolle der Grundrechte für die Fundierung des individuellen Rechtsstatus J. Masing Der Rechtsstatus des Einzelnen im Verwaltungsrecht, in: Hoffmann-Riem/ Schmidt-Aßmann/Voßkuhle (Hrsg.) Grundlagen des Verwaltungsrechts, Band I, 2. Aufl. 2012, § 7, zusammenfassend Rn. 61 ff. Auch aus Art. 36 Grundrechtecharta ergeben sich nach überwiegender Auffassung keine subjektiven Leistungsansprüche im Sinn von Infrastrukturschaffungs- oder -zugangsrechten, vgl. H. D. Jarass GRCh, 2. Aufl. 2013, Art. 36 Rn. 3, 14; J.-C. Pielow in: Tettinger/Stern (Hrsg.) Kölner Gemeinschaftskommentar zur Europäischen Grundrechte-Charta, 2006, Art. 36 Rn. 19; E. Riedel in: Meyer (Hrsg.) Charta der Grundrechte der Europäischen Union, 3. Aufl. 2011, Art. 36 Rn. 2; weiteres Verständnis bei Th. Mann Zugang zu Dienstleistungen von allgemeinem wirtschaftlichem Interesse, in: Heselhaus/Nowak (Hrsg.) Handbuch der Europäischen Grundrechte, 2006, § 34 Rn. 28.

73 Vgl. Fn. 44.

${ }^{74}$ Vgl. bereits M. Fehling Regulierung als Staatsaufgabe im Gewährleistungsstaat Deutschland, in: Hill (Hrsg.) Die Zukunft des öffentlichen Sektors, 2006, 91 (107 ff. mwN in Fn. 108). Anderer Ansicht Gramm Privatisierung (Fn. 42), 190 ff., mit der Kategorie des öffentlichen Gutes; vgl. auch St. Storr Der Staat als Unternehmer, 2001, 103 ff. Siehe wie hier Franzius Gewährleistung (Fn. 3), 78, unter Verweis auf G. F. Schuppert Staatswissenschaft, 2003, 289, 333; vgl. H. Butzer Sicherstellungsauftrag, in: Isensee/Kirchhof (Hrsg.) Handbuch des Staatsrechts, Band 4, 3. Aufl. 2006, § 74 Rn. 7, siehe allerdings auch Rn. 39 ff. So auch die Rechtslage in Bezug auf Art. 14 AEUV, vgl. Ch. Jung in: Calliess/Ruffert (Hrsg.) EUV/AEUV, 4. Aufl. 2011, Art. 14 Rn. 23, dort auch zur politischen Debatte im Vorfeld des Lissabon-Vertrags; St. Wernicke in: Grabitz/Hilf/Nettesheim (Hrsg.) Das Recht der Europäischen Union (Stand 50. Ergänzungslieferung 2013), Art. 14 Rn. 50. Zur entsprechenden Debatte in Bezug auf Art. 33 Abs. 4 GG J. Masing in: Dreier (Hrsg.) GG- Kommentar, Band I, 2. Aufl. 2006, Art. 33 Rn. 62. 
Art. 72 Abs. 2 GG. ${ }^{75}$ Bei vorhandenen Infrastrukturen kann hingegen der Gleichheitssatz verfassungsgestützte Leistungspflichten erzeugen. ${ }^{76}$

Im Umkehrschluss ist auch daran festzuhalten, dass eine einmal formulierte Infrastrukturschaffungspflicht des einfachen Gesetzes keinen Infrastrukturbestandsschutz auslöst, sondern grundsätzlich revisibel im Sinn einer politischen Entscheidbarkeit bleibt: Nachfolgende Generationen haben weder die Pflicht noch das Recht, vorgefundene Infrastrukturen als gegeben zu nehmen; ${ }^{77}$ insoweit kann die Debatte um die Energiewende als allgemeines Beispiel herangezogen werden. ${ }^{78}$ Eine interessante Mischform bildet die prozedurale Rückbindung entsprechender Entscheidungen an Volksentscheide, wie sie seit kurzem das Bremer Landesverfassungsrecht kennt. ${ }^{79} \mathrm{Im}$ Übrigen ist auch der geänderte verfassungsrechtliche Finanzierungsrahmen, der Infrastrukturen nicht mehr unbesehen als

${ }^{75}$ So in aller Klarheit auch $J$. Isensee Staatsaufgaben, in: Isensee/Kirchhof (Hrsg.) Handbuch des Staatsrechts, Band 4, 3. Aufl. 2006, § 73 Rn. 19. Offen dagegen W. Rüfner Daseinsvorsorge und soziale Sicherheit, in: Isensee/Kirchhof (Hrsg.) Handbuch des Staatsrechts, Band 4, 3. Aufl. 2006, § 96 Rn. 16 ff. Zum Verhältnis der Kompetenznorm zu den Vorgaben der sozialpolitischen Raumstruktur J. Kersten DVBl. 2006, 942 (944).

${ }^{76}$ Vgl. P. Kirchhof in: Isensee/Kirchhof (Hrsg.) Handbuch des Staatsrechts, Band 8, 3. Aufl. 2010, $§ 181$ Rn. 72, 238; W. Heun in: Dreier (Hrsg.) GG- Kommentar, Band I, 3. Aufl. 2013, Art. 3 Rn. 82; U. Kischel in: Epping/Hillgruber (Hrsg.) GG-Kommentar 2009, Art. 3 Rn. 80 ff. Die Zuweisung von Infrastruktur kann entsprechend auch als Beispiel für Verteilungsverfahren dargestellt werden, vgl. zur grundrechtlichen Verortung F. Wollenschläger Verteilungsverfahren, 2010, insb. 34 ff.; knapp H. C. Röhl Ausgewählte Verwaltungsverfahren, in: Hoffmann-Riem/Schmidt-Aßmann/Voßkuhle (Hrsg.) Grundlagen des Verwaltungsrechts, Band II, 2. Aufl. 2012, § 30 Rn. 15; allgemein zur Organisation von Verteilungsmodi A. Voßkuhle Die Verwaltung 32 (1999), 21 (29 f.). Auch für die Ausgestaltung der Infrastrukturen können die Grundrechte selbstverständlich relevant werden, vgl. zum Aspekt des „Grundrechts auf Gewährleistung der Vertraulichkeit und Integrität informationstechnischer Systeme“ G. Schulz DUD 2012, 395 ff.; zum Problem der Immissionen durch Infrastruktur am Beispiel des Mobilfunks A. Schmehl/V. Ludewig Jura 2011, 669 (674 ff.).

77 Vgl. fehlgehend BVerwG DVBl. 2009, 1382 ff. - Weihnachtsmarkt. Zur Kritik D. Ehlers DVBl. 2009, 1456 (1456 f.); F. Schoch DVBl. 2009, 1533 (1535 ff.); H. Wißmann Verfassungsrechtliche Vorgaben der Verwaltungsorganisation, in: HoffmannRiem/Schmidt-Aßmann/Voßkuhle (Hrsg.) Grundlagen des Verwaltungsrechts, Band 1, 2. Auflage 2012, 15 Rn. 13; vgl. aber auch A. Katz NVwZ 2012, 405 (408 ff.). Als Ausnahme $\S 11$ AEG, vgl. dazu noch Fn. 121.

${ }^{78} \mathrm{Zu}$ den Verwerfungen bei der Umstellung energiepolitischer Gesamtziele vgl. H. Wißmann Kohlendioxidspeicherung als „Klimaretter für die Kohle“?, in: Gundel/ Lange (Hrsg.) Klimaschutz nach Kopenhagen, 2011, 57 (72 ff.).

${ }^{79}$ Vgl. Art. 42 IV iVm Art. 70 Landesverfassung Bremen, Änderung vom 3.9.2013, GB1. 480 f. (in Bezug auf staatlich beherrschte Unternehmen mit näher spezifiziertem allgemeinem Versorgungsauftrag). 
Quelle neuer Verschuldungsmöglichkeiten ansieht, ${ }^{80}$ ein starkes Indiz gegen festgelegte Infrastrukturschaffungspflichten. ${ }^{81}$

\section{b) Planung und Entscheidungsverfahren}

\section{aa) Die Rolle des Planungsrechts}

Bei der Verwirklichung von Infrastrukturvorhaben sind in der Regel vorgängige allgemeine Planung und eigentliches Genehmigungsverfahren zu unterscheiden..$^{82}$ Die große Chance des Planungsrechts ${ }^{83}$ ist die langfristige, übergreifende Entwicklung von Gesamträumen und Gesamtkontexten jenseits von Einzelprojekten, die vor allem auch "schwachen“

80 Vgl. zu Art. 115 GG für den vorliegenden Zusammenhang J. Kühling DVBl. 2013, 1093 (1100). Näher Ch. Ohler DVB1. 2009, 1265 (1271 f.).

${ }^{81}$ Entsprechend zur Forderung nach einem geschlossenen „Finanzierungskreislauf Straße" stellvertretend W. Kugele N\&R 2010, 91 (93 ff.).

82 Das entwicklungsgeschichtliche Auseinanderfallen von Raumplanung und Planfeststellung (s. o. bei Fn. 32 f.) wird relativiert, wenn man beide als Erscheinungsformen der Außenplanung begreift (im Unterschied zur Binnenplanung als rein staatsinternem Vorgang), so die Begriffsbildung von G. Roellecke DÖV 1994, 1024 (1026 ff.). Zur neueren Entwicklung der sog. projektorientierten Raumordnung, die über eine bloße Koordinierung anderer Planungen zu einer unmittelbaren Einwirkung auf die Vorhabenzulassung übergeht, grundlegend $R$. Wahl FS Sellner 2010, 155 ff. (insb. 159 ff.); ferner M. Wetzel Rechtsfragen einer projektorientierten Raumordnung, 2011, insb. 55 ff., $65 \mathrm{ff}$. O. Rojahn NVwZ 2011, 654 ff.; vgl. R. Steinberg DVBl. 2010, 137 ff.

${ }_{83}$ Vgl. bereits oben Fn. 33, 41, 51. Neben der allgemeinen Raumordnung und der Fachplanung im technischen Sinn ist Planung insbesondere auch im Bereich staatlicher Binnentätigkeit eine näher zu betrachtende Handlungsdimension: Die Landesschulgesetze sehen die Aufstellung von Schulentwicklungsplänen vor, in denen die Schulträger für ihr Gebiet den gegenwärtigen und künftigen Schulbedarf feststellen bzw. prognostizieren sowie Schulstandorte festlegen, vgl. im Überblick A. Leppin NordÖR 1999, 90 ff.; E. Roßner RdJB 1999, 452 ff.; H. Budde/K.-D. Janßen RdJB 2005, 11 ff.; M. Winkler DÖV 2011, 686 ff. Die Schulentwicklungsplanung muss als raumrelevante Planung (insb. Schulstandorte) die Erfordernisse der Raumordnung beachten (vgl. etwa die ausdrückliche Raumordnungsklausel in $§ 145$ Abs. 4 Satz 2 HessSchG; iÜ gilt davon unabhängig § 4 Abs. 1 ROG). Die Planung und Zulassung von Krankenhäusern nach dem Krankenhausfinanzierungsgesetz (KHG) des Bundes und den Krankenhausgesetzen der Länder gehört zur raumbezogenen Fachplanung insoweit, als es um den auf einen Planungsraum bezogenen Einsatz von Ressourcen geht, vgl. W. Köck in: Hoffmann-Riem/Schmidt-Aßmann/Voßkuhle (Hrsg.) Grundlagen des Verwaltungsrechts, Band II, 2. Aufl. 2012, § 37 Rn. 40, 43, 87 ff.; F. Stollmann in: Huster/Kaltenborn (Hrsg.) Krankenhausrecht, 2010, § 4; H. Thomae Krankenhausplanungsrecht, 2006; M. Quaas/R. Zuck Medizinrecht, 2. Aufl. 2008, Rn. 320 ff.; U. Steiner NVwZ 2009, 486 ff.; K. Rennert DVBl 2010, 936 ff.; M. Burgi NVwZ 2010, 601 ff.; zur prozessualen Seite vgl. Ch. Seiler/Th. Vollmöller DVB1. 2003, 235 ff. 
Interessen (namentlich dem Umweltschutz ${ }^{84}$ ) wie überhaupt der Frage nach dem Bedarf ${ }^{85}$ zu einer besseren Position verhelfen kann. ${ }^{86}$ Die offensichtliche Gefahr liegt darin, dass in technokratischem Modus scheinbar entpolitisiert Zwangsläufigkeiten produziert werden, die dann durch konkrete Entscheidungen nur noch nachvollzogen werden können ${ }^{87}$ - als

${ }^{84}$ Zur Rolle des Umweltschutzes in der raumordnerischen Standortplanung von Infrastrukturvorhaben siehe bereits Fn. 35. Die Belange des Umweltschutzes werden flankiert durch altruistische Klagerechte der Umweltverbände; dazu vor allem J. Masing Die Mobilisierung des Bürgers für die Durchsetzung des Rechts, 1997; S. Schlacke Überindividueller Rechtsschutz, 2008; aktuelle Übersichten bei M.-J. Seibert NVwZ 2013, 1040 ff.; W. Porsch NVwZ 2013, 1062 ff.; zu den strukturellen Veränderungen des deutschen Prozessrechts J. Berkemann DVB1. 2011, 1253 ff.; ders. DVBl. 2013, 1137 ff.; M. Hong JZ 2012, 380; K. F. Gärditz Die Verwaltung 46 (2013), 257 ff.

${ }^{85}$ Vom Umweltrecht ausgehende allgemeine Aufbereitung bei I. Appel Staatliche Zukunfts- und Entwicklungsvorsorge, 2005, 58 ff. Für den Bereich der Planfeststellung kritisch Th. Gro $\beta$ VerwArchiv 104 (2013), 1 (15 ff.), der für eine Fortentwicklung zur positiven Nutzenfeststellung als planungsrechtlicher Voraussetzung plädiert. Zum Konzept eines Bedarfserörterungsverfahrens M. Burgi NVwZ 2012, 277 (278 ff.); vgl. zur Koordinierung von Planungsmaßnahmen auch St. Trülzsch/T. Schmidt IR 2012, 325 (326 f.). Zur Bedarfsplanung im Gesundheitsrecht C. Franzius VSSR 2012, 49 (50 ff.). Insbesondere zum Faktor der demographischen Entwicklung vgl. M. Arndt/A. Brauckmann/F. Schwabedal IR 2010, 310 (zu Instrumenten der Nachhaltigkeitsprüfung 311 ff.); F. Brosius-Gersdorf VerwArchiv 98 (2007), 317 ff.; vgl. auch J. Kersten DVB1. 2006, 942 (946 ff.); umfassend ders. Die Verwaltung 40 (2007), 309 (332 ff.); allg. rechtspolitisch W. Kahl DÖV 2009, 2 (9). Gesamtaufbereitung bei W. Kluth und S. Baer Demographischer Wandel und Generationengerechtigkeit, VVDStRL 68 (2009), 246 ff. (einschlägig $275 \mathrm{ff}$.), $290 \mathrm{ff}$.

${ }^{86}$ Zur Unterscheidung von indikativer, influenzierender und imperativer Planung vgl. W. Köck in: Hoffmann-Riem/Schmidt-Aßmann/Voßkuhle (Hrsg.) Grundlagen des Verwaltungsrechts, Band II, 2. Aufl. 2012, 37 Rn. 45 ff. mwN. Zur Orientierung der „traditionellen“ Raumordnung auf den „Binnenraum der Verwaltung“ $R$. Wahl FS Sellner 2010, 155 (155 f.). Konzeptionell zur Gestaltungsaufgabe M. Fehling Verwaltung zwischen Unparteilichkeit und Gestaltungsaufgabe, 2001, insb. 124 ff., 150 ff., 169 ff. mit den Kategorien „politische Gestaltung“, „sachverständige Problemlösung“ und „effizienzorientierte Dienstleistung“. Zur Ergänzung herkömmlicher Planungsvorgänge durch Konzeptpflichten Schmidt-Aßmann Ordnungsidee (Fn. 3), Rn. 6/98 f. Zu den Entwicklungsmöglichkeiten der europäischen Raumentwicklung J. Kersten UPR 2010, $201 \mathrm{ff}$. Für die technische Seite der geplanten Infrastrukturen ist zu beachten, dass hier die transnationale Dimension des Infrastrukturrechts inzwischen auch rechtlich verbindlich ist, wie sich insbesondere in den europäischen Synchronisierungskompetenzen hinsichtlich Interoperabilität und Konnektivität zeigen lässt, vgl. Art. 170 Abs. 2, 194 AEUV. Zum Zusammenhang zwischen Energieplanung und Systemoptimierung $\mathrm{H}$. Krawinkel ZNER 2012, 461 (462).

${ }^{87}$ Allgemein berichtend W. Erbguth DÖV 2012, 821 (822, 825); M. Burgi NVwZ 2012, 277 (277); pointiert $K$. Waechter Diskussionsbemerkung VVDStRL 70 (2011), 342 f. Zum Verständnis von „Planung als Regulierung“ C. Franzius VSSR 2012, 49 
Konsequenz wird gerade in letzter Zeit eine stärkere Beteiligung der Öffentlichkeit bereits in diesem frühen Stadium gefordert. ${ }^{88}$

\section{bb) Entscheidungsverfahren: Planfeststellung}

Typischerweise von der allgemeinen Planungsphase getrennt ist die Entscheidung über das konkrete Projekt, für das in Bezug auf Infrastrukturen das Planfeststellungsverfahren zentrale Bedeutung hat. ${ }^{89}$ Die Grund-

(61 ff.). Zum besonderen Fall des Bundesverkehrswegeplans Th. Groß VerwArchiv 104 (2013), 1 ff., mit Vorschlägen zu einer koordinierenden Neuregelung für die Bundesebene $14 \mathrm{ff}$. Zu den (als Förderungsvoraussetzung fungierenden) „Leitlinien“ für transeuropäische Netze nach Art. 171 AEUV W. Frenz/Ch. Ehlenz IR 2010, 173 ff. Zur Entwicklung der Raumordnungsziele als Hauptinstrument raumordnerischer Steuerung auch M. Ronellenfitsch FS Hoppe, 2000, 355 ff.; S. Paetow in: Erbguth/Kluth (Hrsg.) Planungsrecht in der gerichtlichen Kontrolle, 2011, 179 ff.; ders. FS Wahl, 2011, $511 \mathrm{ff}$. Zur Beteiligung Privater umfassend B. Remmert Private Dienstleistungen in staatlichen Verwaltungsverfahren, 2003, $30 \mathrm{ff}$.

88 Konzeptionell zuletzt J. Ziekow Gutachten D zum 69. DJT, 2012, D 80 ff. Dezidiert für eine Stärkung der Öffentlichkeitsbeteiligung bereits in der Raumordnung etwa W. Erbguth DÖV 2012, 821 (822 ff.). Zu entsprechenden Änderungsvorschlägen jenseits des neuen $\S 25$ Abs. 3 VwVfG (,frühe Öffentlichkeitsbeteiligung") M. Burgi NVwZ 2012, 277 ff.; vgl. auch bereits A. Schink DVBl. 2011, 1377 ff. Gesamtdarstellung der Rechtslage bei M. Fehling BLJ 2012, 92 (93 ff.); zu Strategien der Akzeptanzverbesserung C. Franzius GewArchiv 2012, 225 (228 ff.); insb. zur neuen Rechtslage in Bezug auf den Bundesverkehrswegeplan nach $\S$ 19b, 14i UVPG R. Wulfhorst DVBl. 2012, $466 \mathrm{ff}$. Zu Forderungen der Praxis B. Stuer DVB1. 2012, 885 ff. Als Beispiel für die direkte gesetzliche Entscheidung über die Planerforderlichkeit (hier gemäß § 43 EnWG) vgl. das Energieleitungsausbaugesetz (EnLAG) von 2009, BGBl. I 2870; zum „Bundesbedarfsplan“ § 12e EnWG. Zu entsprechenden „Planungskaskaden“ unter dem Aspekt der Öffentlichkeitsbeteiligung E. Hofmann JZ 2012, 701 (706 ff.). Die Debatte um das NABEG ist dabei teilweise von einer geradezu erstaunlichen Systemtreue geprägt; vgl. W. Erbguth DVBl. 2012, 325 ff.; ders. NVwZ 2012, 326 (328); sowie ders. DVBl. 2013, 274 (276); ähnlich R. Steinberg/M. Wickel/H. Müller Fachplanung, 4. Aufl. 2012, § 7 Rn. 129. Gesamtüberblick bei W. Durner DVBl. 2011, 853 ff.; ders. NuR 2012, 369 ff.; M. Appel UPR 2011, 406 ff.; Ch. Calliess/M. Dross JZ 2012, 1002 ff.; J. GundellK. W. Lange (Hrsg.) Der Umbau der Energienetze als Herausforderung für das Planungsrecht, 2012. Zum Einfluss des NABEG auf die Weiterentwicklung der transeuropäischen Energieinfrastruktur, insbesondere auf den im Oktober 2011 vorgelegten Entwurf einer Verordnung zu Leitlinien für die transeuropäische Energieinfrastruktur H. Schmitz/Ph. Jornitz NVwZ 2012, 332 ff.; inzwischen ist die Verordnung (EU) Nr. 347/2013 des Europäischen Parlaments und des Rates vom 17. April 2013 zu Leitlinien für die transeuropäische Energieinfrastruktur und zur Aufhebung der Entscheidung Nr. 1364/2006/EG und zur Änderung der Verordnungen (EG) Nr. 713/2009, (EG) Nr. 714/2009 und (EG) Nr. 715/2009, Amtsblatt L 115/39, in Kraft getreten.

$89 \S 72$ I 1 VwVfG iVm $\S 17$ ff. FStrG; $\S \S 18$ ff. AEG; $\S 8$ ff. LuftVG; $\S 14$ ff. WaStrG; $\S \S 9$ a Abs. 3 1, 9b Abs. 11 AtG; $\S 28$ Abs. 11 1, $\$ 41$ Abs. 1 PBefG; $\S \S 1$ ff. MBPIG; $§ 68$ Abs. 1 WHG; $\S 41$ Abs. 3 FlurbG; $§ 35$ Abs. 21 KrW-/AbfG; $\S 52$ Abs. 2a 
idee der Planfeststellung - nämlich die förmliche Ordnung mehrseitiger Rechtsverhältnisse durch ein besonderes Verwaltungsverfahren mit dem Abschluss durch eine umfassende Genehmigung - ist dabei wie bereits angedeutet einer der Urtypen des frühmodernen Verwaltungsrechts. ${ }^{90}$

Die weitere Entwicklung und der heutige Stand des Planfeststellungsverfahrens sind dadurch geprägt, dass einerseits das Potential des Verfahrens, das gerade für eine demokratische, grundrechtlich geprägte Rechtsordnung besteht, weiter entfaltet wurde. ${ }^{91}$ Andererseits bestehen durch die

1 BBergG. Grundlegend zum Planungscharakter der Planfeststellung R. Wahl DVBl. 1982, 51 ff.; vgl. ferner mwN W. Durner Konflikte räumlicher Planungen, 2005, 58 ff.; R. Steinberg/M. Wickel/H. Müller Fachplanungsrecht, 3. Aufl. 2012, § 1 Rn. 8 ff.; R. WahllS. Paetow in: Hansmann/Sellner (Hrsg.) Grundzüge des Umweltrechts, 4. Aufl. 2012, Kap. 4 Rn. 18 ff. Für ein Verständnis als reine Zulassungsentscheidung (Kontrollerlaubnis) W. Löwer DVB1. 1981, 528 (529); G. Gaentzsch FS Schlichter, 517 ff. (insb. 525); H. D. Jarass DVB1. 1998, 1202 (1203 f.). Umfassende Aufbereitung der Diskussion bei $H$. Schoen Die Planfeststellung zwischen Kontrollerlaubnis und Planungsentscheidung, 2003. Für stationäre Infrastrukturen sind teilweise gewachsene Bereichsausnahmen zu verzeichnen: Das Krankenhausrecht trifft keine Sonderregeln zur baurechtlichen Zulassung, insb. fehlt die Konzentrationswirkung einer Planfeststellung. Daher wird die baurechtliche Zulassung regelmäßig über die Bauleitplanung (zB Festsetzung eines sonstigen Sondergebiets gem. § 11 Abs. 2 Satz 2 BauNVO; dazu mwN W. Söfker in: Ernst/Zinkahn/Bielenberg/Krautzberger (Hrsg.) BauGB-Kommentar (2013), § 11 BauNVO Rn. 36) und über das baurechtliche Genehmigungsverfahren bewerkstelligt. Auch Schulgebäude werden nicht durch Planfeststellung zugelassen, so dass die Baugenehmigungspflicht nicht aufgrund einer Konzentrationswirkung des Planfeststellungsbeschlusses entfällt. Für die Lage bei Mobilfunkstationen A. Schmehll V. Ludewig Jura 2011, $669 \mathrm{ff}$.

90 Vgl. Fn. 32. Allgemein H. Pünder Verwaltungsverfahren, in: Erichsen/Ehlers (Hrsg.) Allgemeines Verwaltungsrecht, 14. Aufl. 2010, § 13 Rn. 2; plastisch Faber Verwaltungsrecht (Fn. 55), 354 f. unter Rückgriff auf Blümel Die Bauplanfeststellung (Fn. 32). Zuletzt hat M. Fehling BLJ 2012, 92 (92), in der Debatte um Bürgerbeteiligung im Planfeststellungsverfahren daran erinnert, dass das Planfeststellungsverfahren primär dazu dient(e), der Verwaltung Informationen über abwägungsrelevante Informationen zu liefern, dort 94 ff. Darstellung der bisherigen zweistufigen Öffentlichkeitsbeteiligung im Planfeststellungsverfahren; vgl. auch Schmidt-Aßmann Ordnungsidee (Fn. 3), Rn. $6 / 98$ f.

${ }_{91}$ Vgl. bereits Fn. 41. Zur verfassungsrechtlichen Verortung des planerischen Abwägungsgebots grundlegend $W$. Hoppe DVB1. 1964, 165 ff.; vgl. ferner mwN Durner Konflikte (Fn. 89), 301 ff.; W. Erbguth in: ders./Kluth (Hrsg.) Planungsrecht in der gerichtlichen Kontrolle, 2011, 103 ff.; $R$. Bach Die Abwägung gemäß § 1 Abs. 7 BauGB nach Erlass des EAG Bau, 2011, 38 ff.; M. Merkel Die Gerichtskontrolle der Abwägung im Bauplanungsrecht, 2012, 122 f.; aus der Rechtsprechung insofern richtungsweisend BVerwGE 41, 67 (69); BVerwGE 48, 56 (63); BVerwGE 56, 110 (123). Zur Kritik K. $-H$. Ladeur Der Staat 48 (2009), 163 (169 ff.). Zu verfassungsrechtlich und europarechtlich vorgegebenen Verfahrensrechten Überblick bei $H$. Pünder Verwaltungsverfahren, in: Erichsen/Ehlers (Hrsg.) Allgemeines Verwaltungsrecht, 14. Aufl. 2010, § 13 Rn. 7 f., 
Konzentrationswirkung der Genehmigungsverfahren ${ }^{92}$ und die enteignungsrechtliche Vorwirkung ${ }^{93}$ nach wie vor erhebliche Anreize zur Nutzung dieses Instrumentariums. ${ }^{94}$ Infrastrukturvorhaben werden in diesem

12 f. Zur Entwicklung der Erörterung im Planfeststellungsverfahren P. Cancik DÖV 2007, 107 (111 ff.). Insbesondere ist auch die Beteiligung der betroffenen Bürger an der Entscheidungsfindung immer weiter verfeinert worden, ohne damit freilich durchweg friedensstiftende Ergebnisse erzielen zu können. Zum Phänomen „Not in my backyard (NIMBY-Güter)“ $U$. Scheele IR 2012, 247 (248 ff.). Insbesondere für die rechtsstaatliche Dimension einer Integration des Bürgerparadigmas in das europäisierte und internationalisierte Verwaltungsrecht A. v. Arnauld JöR 59 (2011), 497 (510 ff.). Weiter aus der überbordenden aktuellen Diskussion mit übersichtlicher Darstellung der Reformvorschläge bereits $R$. Wulfhorst DÖV 2011, 581 (583 ff.); vgl. W. Erbguth DÖV 2012, 821 ff.; F. Wittreck ZG 2011, 209 (211 ff.); R. Steinberg ZUR 2011, 340 (341 ff.); M. Fehling BLJ 2012, 92 ff.; skeptisch K. F. Gärditz GewArch 2011, 273 (279); H.-G. Henneke DVB1. 2012, 1072 ff. Zum Beispiel Stuttgart 21 stellvertretend Th. Groß DÖV 2011, 510 (512 ff.); A. Schmehl FS Bull, 2011, 347 (348 ff.); C. Franzius GewArchiv 2012, 225 ff.; für die Debatte zum Netzausbau E. Hofmann JZ 2012, 701 (706 ff.).

92 Zur (formellen) Konzentrationswirkung der Planfeststellung $H$. W. Laubinger VerwArch 77 (1986), 77 ff.; R. Wahl/S. Paetow in: Hansmann/Sellner (Hrsg.) Grundzüge des Umweltrechts, 4. Aufl. 2012, Kap. 4 Rn. 27 ff. Zur Konzentrationswirkung allgemein K. Odendahl VerwArch 94 (2003), 222 ff.; F. Wittreck VerwArch 100 (2009), 71 ( 81 ff., 86 ff.). Vgl. zu umweltrechtlichen Konzentrationswirkungen außerhalb des Planfeststellungsrechts K. Schonenbroicher in: Burgi/ders. (Hrsg.) Die Zukunft des Verwaltungsverfahrensrechts, 2010, 82 (84 ff.). Zum umweltrechtlich diskutierten Konzept der integrierten Vorhabengenehmigung W. Kahl/L. Diederichsen NVwZ 2006, 1107 ff.; M. Martini VerwArchiv 100 (2009), 40 (45 ff.); ausführlich zu den europarechtlichen Vorgaben $B$. Welke Die integrierte Vorhabengenehmigung, 2010, 26 ff., 36 ff., dort 72 ff. auch zum Scheitern des Entwurfs von 2008. Zum Zusammenhang zwischen Planfeststellung und Anlagengenehmigung nach $\S 13$ BImSchG M. Fehling Zulassung von Erzeugungsanlagen, in: Schneider/Theobald (Hrsg.) Handbuch zum Recht der Energiewirtschaft, 2003, § 7 Rn. 5 ff., 30 ff.

93 J. Kühling/N. Herrmann Fachplanungsrecht, 2. Aufl. 2000, Rn. 470 ff.; H. D. Jarass DVB1. 2006, 1329 ff. Zur Problematik des vorzeitigen Enteignungsverfahrens nach § 45b Satz 1 EnWG und $\S 27$ Abs. 2 Satz 1 NABEG Ch. Moench/M. Ruttloff NVwZ 2013, $463 \mathrm{ff}$.

94 Zur Diskussion, inwieweit privatnützige Planfeststellungen besonderen Voraussetzungen unterliegen J. Kühling/N. Herrmann Fachplanungsrecht, 2. Aufl. 2000, Rn. 285 ff.; H. D. Jarass Die Planfeststellung privater Vorhaben, 2003; R. Steinberg/ M. Wickel/H. Müller Fachplanungsrecht, 3. Aufl. 2012, § 1 Rn. 22 ff. Die Rechtsprechung hat die Unterscheidung zwischen öffentlichen und privatrechtlichen Nutzungen erheblich relativiert; vgl. insb. BVerwGE 128, 358 ff. - Mühlenberger Loch; vgl. bereits R. Wahl/J. Dreier NVwZ 1999, 606 (609 f.). Zum Themenkreis Enteignung zugunsten Privater allgemein weiter $O$. Depenheuer in: v. Mangoldt/Klein/Starck (Hrsg.) Kommentar zum Grundgesetz, 6. Aufl. 2010, Art. 14 Rn. 428 ff.; H. D. Jarass, in: ders./Pieroth, GG, 12. Aufl. 2012, Art. 14 Rn. 80a; J. Wieland in: Dreier (Hrsg.) GG, 3. Aufl. 2013, Art. 14 Rn. 120; W. Leisner Eigentum, in: Isensee/Kirchhof (Hrsg.) Handbuch des Staatsrechts, Band 8, 3. Aufl. 2010, § 173 Rn. 221; speziell zur Energiewirtschaft 
Zusammenhang zu Recht als übergreifende, multipolare Entscheidungen begriffen, die nicht adäquat durch adressatenbezogene Einzelgenehmigungen erfasst werden könnten. ${ }^{95}$ Andererseits stellt sich das Planfeststellungsrecht durch seine Orientierung am Verwaltungsakt als im Grundzug konservativer, auf statische Verbindlichkeit gerichteter Entwurf dar. ${ }^{96}$

\section{cc) Insbesondere: Modifizierungen für öffentliche Infrastrukturen}

Es kennzeichnet die Überlastung des Planfeststellungsverfahrens, dass insbesondere für Infrastrukturprojekte mit dem Instrument der Plangenehmigung eine aus Effizienzgründen verschlankte Fassung geschaffen wurde. ${ }^{97}$ Damit ist gerade dort, wo die multipolare Lage und die Vielzahl der Abwägungsfaktoren den Aufwand des Verfahrens begründen würden, der legitimationsstiftende Teil weitgehend weggekürzt. Mit Abweichungen im Detail gilt dies etwa für das Eisenbahn-, Fernstraßen- und Wasserstraßenrecht, aber auch für das Energierecht. ${ }^{98}$

BVerwGE 116, 365; allgemeiner BVerwGE 71, 108 (124 f.); 87, 241 (247 f.). Zu weiteren Fallkonstellationen St. Muckel BayVBl. 2011, 225 ff. Siehe ferner W. Schmidbauer Enteignung zugunsten Privater, 1989; S. Pommer Bahnreform und Enteignung, 2002; A. Jagst Enteignung zugunsten privatisierter Flughäfen, 2012. Zur Duldungspflicht von Grundstückseigentümern nach dem TKG 2012 („Hausstich“) M. Freund/T.-L. Bary NVwZ 2012, 1504 ff.; B. Kind/M. Schramm N\&R 2012, 140 (147 f.).

${ }_{95}$ Ausführliche Übersicht über die Abschnitte des Verfahrens unter besonderer Betonung des Infrastrukturcharakters $H$. Pünder Planfeststellungsverfahren, in: Erichsen/Ehlers (Hrsg.) Allgemeines Verwaltungsrecht, 14. Aufl. 2010, § 15 Rn. 4 ff.

${ }_{96}$ Zur Verortung im System der Verwaltungsakte Ch. Bumke in: Hoffmann-Riem/ Schmidt-Aßmann/Voßkuhle (Hrsg.) Grundlagen des Verwaltungsrechts, Band II, 2. Aufl. 2012, $\$ 35$ Rn. 103. Zur Verfahrensdimension zuletzt eingehend M. Fehling BLJ 2012, 92 (95 ff.) Anderes gilt freilich für die Entwicklung der gerichtlichen Kontrollmaßstäbe, vgl. unten Fn. 102.

${ }_{97}$ Die Plangenehmigung wurde zunächst im Fachplanungsrecht vorgesehen (WHG, WaStrG, FlurbG). Das Verkehrswegeplanungsbeschleunigungsgesetz vom 16. Dezember 1991 (BGBl. I 2174) weitete ihren Anwendungsbereich im Straßenrecht aus; das Planvereinfachungsgesetz vom 26. Juni 1993 (BGBl. I 2123) erstreckte sie auf weitere Fachplanungsgesetze (zB LuftVG, PBefG). Das Genehmigungsbeschleunigungsgesetz vom 12. Dezember 1996 (BGBl. I 1354) hat die Plangenehmigung mit der in $\S 74$ Abs. 6 VwVfG getroffenen Regelung dann in das allgemeine Planfeststellungsrecht eingeführt. Vgl. dazu H. D. Jarass DVBl. 1997, 795 (796 ff.); zur Entwicklung auch H. J. Bonk/ W. Neumann in: Stelkens/Bonk/Sachs (Hrsg.) VwVfG-Kommentar, 7. Aufl. 2008, § 74 Rn. 222; kritisch insb. W. Erbguth DÖV 2009, 921 ff.; vgl. zu den raumordnungsrechtlichen Sonderregeln $R$. Wahl FS Sellner 2010, 155 (159 ff.).

${ }_{98}$ Grundsätzlich entfällt die enteignungsrechtliche Vorwirkung bei der Plangenehmigung, vgl. ausdrücklich $\S 74$ VI 2 VwVfG. Intrikaterweise wird dann allerdings gerade für die hier relevanten öffentlichen Infrastrukturen die Plangenehmigung der Planfeststellung gleichgestellt, so etwa in $\S 22$ Abs. 2 AEG, § 19 Abs. 2 FStrG, $\S 30$ PBefG, 


\section{dd) Strukturelle Überlastung}

Gerade durch diese Modifizierung tritt die strukturelle Schwäche des Planfeststellungsverfahrens besonders klar hervor: Es überschätzt letztlich den abschließenden Charakters rechtlicher Entscheidungen und konzentriert sich also ganz auf diesen einen Moment mit der Folge völliger Überlastung, was zu entsprechenden Ausweichbewegungen führt. Insbesondere sind die notwendig folgenden Abschnitte bis zur Realisierung eines Vorhabens (durch den Grundsatz der umfassenden Konfliktbewältigung ${ }^{99}$ und das Recht der Nebenbestimmungen) nicht ausreichend begleitet. ${ }^{100}$ Das berücksichtigt nicht ausreichend, dass das Risiko des Scheiterns nicht allein bei einem privaten Vorhabensträger liegt, sondern auch die Allgemeinheit trifft. Und dass gerade hier Verfahrensfehler weitgehend als irrelevant eingestuft werden, ist ein besonders trauriges Beispiel für ein gegenseitiges downsizing von allgemeinem und besonderem Verwaltungsrecht. ${ }^{101}$ Gleiches gilt für die Verkürzung des Rechtsschutzes, bei

$\S 28$ Abs. 2 LuftVG, $\$ 45$ Abs. 2 EnWG. Das NABEG wählt einen anderen Weg, indem es in $\S 18$ allein die Planfeststellung, nicht aber die Plangenehmigung vorsieht, dafür aber die enteignungsrechtliche Vorwirkung vorverlegt; zur umstrittenen Frage der Verordnungsermächtigung des Bundes zur Beauftragung der BNA zur Durchführung von Planfeststellungsverfahren nach $\S 2$ II M. Appell A. Erding NVwZ 2012, 343 (346 f.).

99 Zum Bereich der Straßenplanung BVerwGE 57, 297 (301 f.); 102, 331 (346).

${ }^{100}$ H. Pünder Planfeststellungsverfahren, in: Erichsen/Ehlers (Hrsg.) Allgemeines Verwaltungsrecht, 14. Aufl. 2010, § 15 Rn. 23 f.

${ }^{101}$ Abwägend Ch. Bumke Relative Rechtswidrigkeit, 2004, 208 ff.; F. Schoch Gerichtliche Verwaltungskontrollen, in: Hoffmann-Riem/Schmidt-Aßmann/Voßkuhle (Hrsg.) Grundlagen des Verwaltungsrechts, Band III, 2. Aufl. 2013, § 50 Rn. 306; vgl. zur allgemeinen Lage im Rechtsvergleich M. Fehling Eigenwert des Verfahrens im Verwaltungsrecht, VVDStRL 70 (2011), 278 (292 ff.). Gegen das „Planerhaltungsrecht“ zuletzt $W$. Erbguth DÖV 2012, 821 (824 f.); insbesondere in Bezug auf die Sicherung der Öffentlichkeitsbeteiligung E. Hofmann JZ 2012, 701 (709 f.). Umfassende Darstellung der frühen Entwicklung bei $R$. Käß Inhalt und Grenzen des Grundsatzes der Planerhaltung, 2002, 59 ff. Vgl. auch H. D. Jarass DVB1. 1997, 795 (800 ff.); G. Gaentzsch DVBl. 2000, 741 ff.; ders. UPR 2001, 201 ff.; zum Bauplanungsrecht W. Hoppe DVB1. 1996, 12 ff.; H. Sendler DVB1. 2005, 659 ff.; U. Stelkens UPR 2005, 81 ff. Zur Planerhaltung im Raumordnungsrecht Ch. Wiggers Planerhaltung im Recht der Raumordnung, 2003. $\mathrm{Zu}$ den Anforderungen des Unionsrechts an Planerhaltungsvorschriften $M$. Kment DÖV 2006, 462 ff.; ders. DVB1. 2007, 1275 ff. Kritisch gegen einfach-rechtliche Planerhaltungsnormen mit Blick auf die ,grundrechtssichernde Funktion“ der Verfahrensvorschriften im Planungsrecht $W$. Köck in: Hoffmann-Riem/Schmidt-Aßmann/Voßkuhle (Hrsg.) Grundlagen des Verwaltungsrechts, Band II, 2. Aufl. 2012, § 37 Rn. 116. Siehe zu diesem Komplex auch E. Schmidt-Aßmann in: Hoffmann-Riem/ders./Voßkuhle (Hrsg.) Grundlagen des Verwaltungsrechts, Band II, 2. Aufl. 2012, § 27 Rn. 105 ff.; ferner F. Hufen/T. Siegel Fehler im Verwaltungsverfahren, 5. Aufl. 2013, Rn. 911 ff. Zur parallelen Diskussion zu $\S \S 214,215$ BauGB siehe die Nachweise bei $U$. Battis in: ders./Krautzberger/Löhr (Hrsg.) BauBG, 11. Aufl. 2009, Vorb §§ 214-216 Rn. 12. 
der sich die eingeschränkte materielle Kontrolle inzwischen mit einer erstund letztinstanzlichen Zuständigkeit des BVerwG verbindet. ${ }^{102}$

Insgesamt bleibt so der politisch-gestaltende Charakter von Infrastrukturentscheidungen hinter der Fassade von Planfeststellungsverfahren eher verborgen. Damit wird aber nicht nur Scheinrationalität, sondern auch Scheinlegitimation erzeugt. Darauf wird unter dem Stichwort des politischen Charakters von Infrastrukturvorhaben zurückzukommen sein.

\section{Bereitstellung}

\section{a) Grundsatz der Typenwahlfreiheit-Herausforderung Vertragsgestaltung}

Bei der Bereitstellung von Infrastrukturen, die der Entscheidung über das „ob“ nachfolgt, bildet die physische Herstellung regelmäßig die nächste Etappe, etwa durch den Bau von Straßen, Leitungen oder bestimmten Gebäuden. ${ }^{103}$ Soweit es dabei um Einrichtungen geht, die dann ohne weiteres der Allgemeinheit als Gemeingebrauch ${ }^{104}$ zur Verfügung stehen, kann festgehalten werden, dass Träger einer solchen Herstellung sowohl die öffentliche Hand sein kann (wie bei Straßen oder Schulen) wie auch Einrichtungen des Dritten Sektors oder private Unternehmen sein können.

Typischerweise beauftragt der Träger private oder gemischt-wirtschaftliche Unternehmen mit bestimmten Herstellungsleistungen. ${ }^{105}$ Die Ent-

$102 \S 50$ I Nr. 6 VwGO, zur Ausweitung nach dem Infrastrukturplanfeststellungsbeschleunigungsgesetz kritisch $H$. Pünder Planfeststellungsverfahren, in: Erichsen/Ehlers (Hrsg.) Allgemeines Verwaltungsrecht, 14. Aufl. 2010, § 15 Rn. 27. Zum Instanzenzug einschlägig F. Schoch Gerichtliche Verwaltungskontrollen, in: Hoffmann-Riem/ Schmidt-Aßmann/Voßkuhle (Hrsg.) Grundlagen des Verwaltungsrechts, Band III, 2. Aufl. 2013, $\S 50$ Rn. 330 ff., insb. Rn. 336 f. mit Hinweis auf fehlende „Lernprozesse“. Allgemein zum Kontrollstandard im Planungsrecht W. Hoppe FG 25 Jahre BVerwG, 1978, 295 ff.; ders. FS Menger, 1985, 747 ff.; W. Blümel Planung und Verwaltungsgerichtsbarkeit, 1997, insb. 13 ff.; M. Ronellenfitsch FS Blümel, 1999, 497 ff.; H. SchulzeFielitz FS Hoppe, 2000, 997 ff.; W. Erbguth in: ders./Kluth (Hrsg.) Planungsrecht in der gerichtlichen Kontrolle, 2012, 103 ff.; aus verfassungsgerichtlicher Perspektive W. Hoppe FG 25 Jahre BVerfG, 1976, 663 ff.; M. Bertrams FS Hoppe, 2000, 975 ff.

${ }^{103}$ Freilich gibt es insoweit Ausnahmen: Eine neue Einrichtung kann auch bestehende Infrastrukturen nutzen, so der Rettungsdienst das allgemeine Straßennetz und in der Regel die Anbindung an vorhandene Krankenhäuser. Vgl. zum Rettungsdienst als Infrastruktur Fn. 66.

104 § 7 FStrG; § 6 WaStrG iVm Rechtsverordnung; § 59 Abs. 1 BNatSchG; $\S 14$ BWaldG. Vgl. U. Mager/L. Sokol Jura 2012, 913 ff. Weiter zur Verknüpfung mit Infrastrukturdiensten bei Fn. $109 \mathrm{ff}$.

${ }^{105} \mathrm{Zu}$ Gestaltungsmöglichkeiten der Kommunen siehe J. Hellermann in: Hoppe/ Uechtritz/Reck (Hrsg.) Handbuch kommunale Unternehmen, 3. Aufl. 2012, § 7, insb. Rn. $10 \mathrm{ff}$. 
scheidung für Infrastruktur ist in dieser Phase in erster Linie eine Entscheidung zur Förderung der betreffenden (Bau-) Dienstleister. ${ }^{106}$ Daraus folgt als Hauptaufgabe des Vergaberechts, die Zuteilung öffentlicher Mittel fair zu gestalten. ${ }^{107}$ Dabei sind Vertragsgestaltung und -abwicklung für die spezifischen Anforderungen des Infrastrukturrechts bisher nicht genügend ausgearbeitet, weil sie zu stark auf punktuelle Vereinbarungen statt auf moderierte Dauerbeziehungen setzen. $\mathrm{Zu}$ zielen wäre hier in intradisziplinärer Offenheit auf neue Mischmodelle, die den multipolaren Zielcharakter solcher Vereinbarungen stärker gewichten und präziser erfassen, namentlich die berechtigten Interessen der infrastrukturabhängigen Allgemeinheit, sei es durch Vertragsstrafen, sei es durch Kündigung. ${ }^{108}$

${ }^{106}$ Knapp H. Schulze-Fielitz Grundmodi der Aufgabenwahrnehmung, in: Hoffmann-Riem/Schmidt-Aßmann/Voßkuhle (Hrsg.) Grundlagen des Verwaltungsrechts, Band I, 2. Aufl. 2012, § 12 Rn. 139 f. Nach Evaluation des Bundesrechnungshofs wird bei $85 \%$ der durch Bundesministerien bzw. nachgeordnete Behörden durchgeführten Maßnahmen auf eine Wirtschaftlichkeitsprüfung verzichtet, Präsident des Bundesrechnungshofes als Bundesbeauftragter für die Wirtschaftlichkeit in der Verwaltung, Anforderungen an Wirtschaftlichkeitsuntersuchungen finanzwirksamer Maßnahmen nach $§ 7$ Bundeshaushaltsordnung, 2013, 5.

107 Zum Doppelauftrag des Vergaberechts J. Ziekow Öffentliches Wirtschaftsrecht, 2. Aufl. 2010, §9 Rn. 1; S. Storr in: Ruthig/ders. Öffentliches Wirtschaftsrecht, 3. Aufl. 2011, § 10 Rn. 1024 ff.; H. Pünder in: Ehlers/Fehling/ders. (Hrsg.) Besonderes Verwaltungsrecht, Band I, 3. Aufl. 2012, § 17 Rn. 1 ff.; P. M. Huber in: Schoch (Hrsg.) Besonderes Verwaltungsrecht, 15. Aufl. 2013, Rn. 278 f. Umfassend auch zum folgenden P. F. Bultmann Beihilfenrecht und Vergaberecht, 2004, insb. 93 ff. Zu den Transparenzanforderungen vor dem Maßstab des IFG M. Rossi/M. Vogt Jahrbuch Informationsfreiheit und Informationsrecht, 2011, 61 ff. Vgl. weiter H. Schulze-Fielitz Grundmodi der Aufgabenwahrnehmung, in: Hoffmann-Riem/Schmidt-Aßmann/Voßkuhle (Hrsg.) Grundlagen des Verwaltungsrechts, Band I, 2. Aufl. 2012, § 12 Rn. 142 ff. Insbesondere zu den europarechtlichen Vorgaben im Vergaberecht $J$. Ziekow Öffentliches Wirtschaftsrecht, 2. Aufl. 2010, $\$ 9$ Rn. 2 ff.; ferner S. Hertwig Praxis der öffentlichen Auftragsvergabe, 4. Aufl. 2009, Rn. 26 ff.; zur Verzahnung mit dem kommunalen Wirtschaftsrecht Th. Mann NVwZ 2010, 857 ff.; vgl. umfassend zuletzt Siebler Privilegierung (Fn. 42), $179 \mathrm{ff}$.

$108 \mathrm{Zu}$ mehrseitigen (Verwaltungs-)Verträgen grundlegend F. Reimer VerwArchiv 94 (2003), 543, insb. 562 ff.; zu Umgehungsmöglichkeiten vergaberechtlicher Anforderungen durch Vertragsgestaltung $V$. Poschmann Vertragsänderungen unter dem Blickwinkel des Vergaberechts, 2010. Allgemein zur intradisziplinären Herausforderung D. Ehlers Die Verwaltung 20 (1987), 373 (378 ff.); Ch. Kirchner, H. Trute, W. Hoffmann-Riem, alle in: Hoffmann-Riem/Schmidt-Aßmann (Hrsg.) Öffentliches Recht und Privatrecht als wechselseitige Auffangordnungen, 1996, 63 ff., 167 ff., 261 ff.; H. Wißmann FS Berg, 2011, 556 (561 ff.); zum Verbund zwischen Zivilrecht und Öffentlichem Recht insbesondere M. Burgi Rechtsregime, in: Hoffmann-Riem/Schmidt-Aßmann/Voßkuhle (Hrsg.) Grundlagen des Verwaltungsrechts, Band I, 2. Aufl. 2012, § 18 Rn. 34 ff.; weiter Hellermann Schutz der Verbraucher (Fn. 72), 372 ff. Die institutionelle Aufstellung der Vergabekammern wäre ein Ansatzpunkt für eine reflektierte Zusammenarbeit zwischen 


\section{b) Insbesondere: Private Bereitstellung von Infrastruktur als Vorleistung privilegierter Nutzung - Das Problem von Dienst und Netz}

\section{aa) Anreize für private Investitionen in öffentliche Infrastrukturen}

Wenn öffentliche Infrastrukturen von privaten Investoren eingerichtet werden sollen, müssen hinreichend sichere Anreize für die Investition entweder durch Zuschüsse, Umlagen oder durch sichere Abnahme der entsprechenden Dienstleistungen geschaffen werden. ${ }^{109}$ Das Beihilfenrecht muss hier den Wettbewerbsschutz sichern, dabei aber auch den Unterschied $\mathrm{zu}$ allgemeinen Industriehilfen berücksichtigen. ${ }^{110}$ Insbesondere

öffentlichem und privatem Recht, vgl. zuletzt C. F. Germelmann DÖV 2013, 50 (54 ff.). Vgl. zum Eigenkapitalbeitrag bei der Gründung von Projektgesellschaften Ch. Koenig/ M. Hellstern IR 2010, 345 ff. Vgl. aber auch zur Verteilung realer Handlungsmacht vor Ort H. Treiber FS Faber, 2007, 281 (292 ff.).

109 Ausgehend vom Medienrecht M. Droege Steuerung durch finanzielle Anreize, finanzieller Ausgleich für regulatorische Markteingriffe, BLM Schriftenreihe Band 99, 2011, 97 ff. Einschlägig zu den „Leitlinien“ für transeuropäische Netze als Zuschussvoraussetzungen nach Art. 171 AEUV W. Frenz/Ch. Ehlenz IR 2010, 173 ff. Effizienz- und Wirtschaftlichkeitsregeln setzen hier bei der Kostenermittlung die einschlägigen Grenzen; aktueller Überblick bei J. Kühling DVB1. 2013, 1093 (1100 f.), insbesondere mit Blick auf die TKG-Novelle 2012; vgl. auch T. Fetzer WiVerw 2010, 145 (147 ff.); zum Energierecht $A$. Glaser DVB1. 2012, 1283 (1287 ff.); zum Wahlrecht „Gebühr/Entgelt“ im Privat-Straßenbaurecht E. Gawel IR 2011, 29 (30 ff.). Bei Abnahmepflichten für die auf der Infrastruktur erzeugten Dienstleistungen entstehen (mindestens) Dreieckskonstellationen, was nach aller Erfahrung zu dauernder Nachsteuerung bei der Entgeltregulierung führt, vgl. zu der entsprechenden Debatte A. Baer Abnahmepflichten und Vergütungspflichten in der Energiewirtschaft, 2010, 34 ff., 42 ff.; zur aktuellen Judikatur W.-A. Achilles REE 2012, 138 ff. Allgemein zur Refinanzierung von Infrastrukturinvestitionen aus regulierten Entgelten typologisierend M. Schäfer/P. Reimer VerwArchiv 102 (2001), 431 ff.; zur Unterscheidung von Sachverantwortung und Finanzierungslast bei öffentlichen Aufgaben in privater Hand H. Kube Die Verwaltung 41 (2008), 1 (26 ff.). Auch das Instrument der bewussten Regulierungsfreistellung kann Anreiz sein, vgl. M. Wegner Regulierungsfreistellungen für neue Elektrizitäts- und Erdgasinfrastrukturen, 2010, insb. zu ,access holidays“ 68 ff.; zur „,nicht-fiskalischen Infrastrukturfinanzierung" am Beispiel des ÖPNV O. Mietzsch IR 2010, 270 ff.; zur Kofinanzierung von Eisenbahninfrastrukturprojekten $H$. Gersdorf ZG 2011, $248 \mathrm{ff}$.

${ }_{110}$ Vgl. allgemein Bultmann Beihilfenrecht (Fn. 107), 21 ff., zum Zusammenhang mit dem Vergaberecht 139 ff. Zum Problem der Direkthilfen bei Dienstleistungen von allgemeinem wirtschaftlichem Interesse G. Britz ZHR 169 (2005), 370 ff. In Bezug auf Infrastrukturen zuletzt am Beispiel der Seehäfen C. Jennert/S. Eitner EuZW 2013, 414. Zur „Flughafeninfrastrukturfinanzierung“ A. Seidenspinner ZLW 60 (2011), 569 ff. Überblick über die ältere Praxis der EU-Kommission U. Karpenstein/G. Schiller ZHR 172 (2008), 81 (86 ff.); zur Überschneidung von Kartell- und Beihilfenrecht A. MartinEhlers EuZW 2010, 287 ff. Für die Lage der Kommunen grundlegend $O$. Dörr/D. Heinemann KommJur 2006, 41 ff., 89 ff.; dazu zuletzt W. Pauly/N. Jedlitschka DVBl. 2012, $1269 \mathrm{ff}$. 
können Konzessionen ein naheliegender Weg sein, entsprechende Anreize zu schaffen ${ }^{111}$ - womit zugleich die Gewährleistung eines angemessenen allgemeinen Zugangs und also die Entgeltregulierung sowie die Durchsetzung der „Netzneutralität" als unabweisbare Folgefragen verbunden sind. ${ }^{112}$

\section{bb) Verpflichtung zur Öffnung der Infrastruktur für Wettbewerber}

Ausnahmsweise können Eigentümer von Infrastrukturen gesetzlich verpflichtet sein, diese für andere, insbesondere für Wettbewerber zu öffnen. Das gilt namentlich dort, wo sogenannte „natürliche Monopole“ herrschen. ${ }^{113}$ Hier entstehen eigentumsrechtlich bedingte Ausgleichspflichten, um die Investitionskosten in diesem Fall nicht von Nutzern, sondern von Wettbewerbern zu erlösen. ${ }^{114}$ Ein zukunftsfähiges Infrastrukturrecht

111 Hier bleibt das Betriebsrisiko beim Unternehmer, die Konsequenz ist in der Regel die Vorverlagerung des Wettbewerbs auf die Vergabe dieser Konzession, vgl. zum „Wettbewerb um den Markt“ Franzius Gewährleistung (Fn. 3), 493 ff. Eine Herausforderung bildet (vergaberechtlich) insbesondere das Recht der Dienstleistungskonzessionen, vgl. M. Nettesheim EWS 2007, 145 ff.; Glöckner Infrastrukturverantwortung (Fn. 45), 95 ff.; zur Abgrenzung zwischen Dienstleistungskonzession und Dienstleistungsauftrag EuGH, Urteil vom 10.9.2009, Rs. C-206/08 - Eurawasser; aus der Lit. etwa M. Sitsen IR 2009, 223 ff.; zuletzt insb. zu den europarechtlichen Implikationen $P$. F. Bultmann NVwZ 2011, 72 ff.; Ch. Braun EuZW 2012, 451 ff. Vgl. allgemein mit Beispielen R. Stober in: Wolff/Bachof/ders./Kluth, Verwaltungsrecht II, 7. Aufl. 2010, § 93 Rn. 27.

112 Zur Netzneutralität im Sinne eines gleichberechtigten Zugangs zu Monopolnetzen siehe allgemein M. Fehling in: ders./Ruffert (Hrsg.) Regulierungsrecht, 2010, § 20 Rn. 5 ff.; M. Martini VerwArchiv 102 (2011), 315 ff.; ferner J. Ruthig in: ders./Storr, Öffentliches Wirtschaftsrecht, 3. Auf1. 2011, § 6 Rn. 585 ff. Grundlegend bereits J. Kühling Sektorspezifische Regulierung in den Netzwirtschaften, 2004, 182 ff. Siehe aktuell den Verordnungsentwurf der Europäischen Kommission vom 11.09.2013, dort insb. Art. 23, online abrufbar unter http://ec.europa.eu/transparency/regdoc/rep/1/2013/DE/ 1-2013-627-DE-F1-1.Pdf\#page=58 (zuletzt abgerufen am 19.09.2013).

${ }^{113}$ Kartellrechtliche Begründung bei V. Emmerich FS Söllner, 2000, 273 ff.; vgl. auch ders. Kartellrecht, 12. Aufl. 2012, § 27 Rn. 102 ff. mwN; zur Problematik des Begriffs M. Leschke in: Fehling/Ruffert (Hrsg.) Regulierungsrecht, 2010, § 6 Rn. 54 ff. $\mathrm{mwN}$. Vgl. etwa für den Zugang zu Gasspeichern S. Fischerauer Regulierung des Zugangs zu Speicheranlagen, 2010, insb. $85 \mathrm{ff}$.

${ }_{114}$ Für eine eigentumsrechtliche Entschädigungspflicht im Falle der strukturell vergleichbaren Inpflichtnahme privater Unternehmer zur Wahrnehmung öffentlicher Aufgaben H.-J. Papier in: Maunz/Dürig (Hrsg.) GG-Kommentar, Loseblatt, 68. Lfg. 2013, Art. 14 Rn. 378i f.; M. Fehling in: ders./Ruffert (Hrsg.) Regulierungsrecht, 2010, § 20 Rn. 10 ff. Gleiches gilt dem Grunde nach auch dort, wo die öffentliche Hand Infrastrukturen geschaffen hat und diese nun außerhalb des Gemeingebrauchs für Dienstleistungen ,auf dem Netz“ zur Verfügung stellt. 
muss dabei Investitionszeiträume absichern - darüber hinaus freilich ist „unumkehrbare“ Planungssicherheit eine Chimäre. ${ }^{115}$

Intrikat wird die Zugangsverpflichtung, wo die Eigentümer selbst ebenfalls das Netz nutzen, um Dienste anzubieten, weil über feinste Justierungen des Entgelts die Position der einen oder der anderen Seite verzerrt werden kann. Das Regulierungsverwaltungsrecht, das sich mit diesen Mischkonstellationen zu beschäftigen hat, soll den Grundsatz des diskriminierungsfreien Wettbewerbs durch Zugangsregulierung und Preisregulierung durchsetzen und hat sich dabei für betriebswirtschaftlich angeleitete Modelle der Marktsimulation entschieden, was bei realistischer Betrachtung nicht über den normativen Gehalt auch solcher Regulierungsentscheidungen hinwegtäuschen kann. ${ }^{116}$

Als rechtliche Grundfrage bleibt, ob es ein Regelmodell gibt, nach dem Dienst und Netz grundsätzlich zu trennen sind. Ordnungspolitisch mag eine solche Trennung vielfach Sinn machen, weil sie die angesprochenen Zugangsprobleme entschärft; auch dürfen Einheitsmodelle nicht als quasi unabänderliches Erbe staatlicher Versorgungsstrukturen betrachtet werden. Ein verfassungsrechtlicher oder primärrechtlicher Grundsatz der institutionellen Entflechtung ist aber abzulehnen: ${ }^{117}$ Zunächst einmal ist schon nicht richtig, dass Dienste auf der Infrastruktur stets durch Private oder überhaupt marktförmig zu erbringen sind.118 Und auch soweit

115 Allgemein zu diesem Problem O. Lepsius Nachhaltigkeit und Parlament, in: Kahl (Hrsg.) Nachhaltigkeit als Verbundbegriff, 2008, 326 (333 ff.). Abgrenzung des Regelungsstands zwischen Telekommunikationsrecht und Energierecht bei J. Kühling DVB1. 2013, 1093 (1100 f.). Zu Mehrjahresverträgen im Bereich der BundesfernstraBeninfrastruktur W. Kugele N\&R 2010, 91 ff.; zur Lage im Bereich der Bahn vgl. die Leistungs- und Finanzierungsvereinbarung zwischen Bundesregierung und Deutscher Bahn, abrufbar online beim Eisenbahn-Bundesamt http://www.eba.bund.de. Zur Verlängerung siehe Pressemitteilung des BMVBS vom 06.09.2013, Lfd. Nr. 201/2013. Zum aktuellen europarechtlichen Hintergrund Ch. Lerche N\&R 2013, 27 (30). Vgl. im Überblick H. Gersdorf ZG 2011, 248 ff.; zur Unterscheidung der Lage zwischen Fernverkehr und Nahverkehr ders. DVBl. 2010, 746 ff. Vgl. ansonsten zur Finanzierung von Infrastrukturen durch Zuschüsse bereits Fn. $109 \mathrm{ff}$.

116 J. Masing/H. Wißmann in: Wilms/Masing/Jochum (Hrsg.) TKG, 2006, § 30 Rn. 29 ff. Die Legitimation der Ergebnisse soll institutionell durch die Unabhängigkeit der Bundesnetzagentur erhöht werden. Vgl. zu dieser Debatte allgemein Fn. 152.

117 Vgl. bereits $R$. Scholz Entflechtung und Verfassung, 1982, insb. 19 ff., 83 ff. Ebenso Ch. Möllers/C. Schäfer Verfassungs- und bilanzrechtliche Prüfung des Gesetzentwurfs „Kapitalprivatisierung Deutsche Bahn AG“ des Bundesministeriums für Verkehr, Bau und Stadtentwicklung, 2007, $9 \mathrm{f}$.

118 Allgemein zu den unterschiedlichen Erscheinungsformen von Wettbewerb typologisierend $J$. Kersten Herstellung von Wettbewerb als Verwaltungsaufgabe, VVDStRL 69 (2010), 288 (290 ff.). Umfassend Storr Staat (Fn. 74), 152 ff. Vgl. für den kommunalen Bereich im Einzelnen Glöckner Infrastrukturverantwortung (Fn. 45), 92 ff.; W. Kluth 
Markt und Wettbewerb vorgegeben sind, folgt daraus keine allgemeine Vorgabe, passgenaue Teilnehmer von Teilmärkten „um“ und ,im“ Wettbewerb zu konfigurieren. ${ }^{119}$ Anderes gilt nur als konkrete sekundärrechtliche bzw. gesetzliche Anordnung des „Unbundling“. ${ }^{120}$

\section{Pflege der Infrastruktur}

Ein auf die Zukunft ausgerichtetes Infrastrukturrecht muss schließlich die Pflege, also die Erhaltung und die nachlaufende Ertüchtigung bestehen-

Kommunalwirtschaftliche Aktivitäten als Dienste von allgemeinem wirtschaftlichem Interesse, in: Mann/Püttner (Hrsg.) Handbuch der kommunalen Wissenschaft und Praxis, Band 2, 3. Aufl. 2011, 39 Rn. 7 ff.; näher für das offene Arrangement des Art. 106 Abs. 2 AEUV am Beispiel des Abfallrechts M. Reese/H.-J. Koch DVBl. 2010, 1393 (1396 ff.). Selbst eine so straffe Vorgabe wie die des Art. 170 Abs. 2 AEUV, wonach der Beitrag der EU zum Auf- und Ausbau transeuropäischer Netze „im Rahmen eines Systems offener und wettbewerbsorientierter Märkte" erfolgt, führt nicht zu einem Zwang zu Liberalisierung und Privatisierung, vgl. Ch. Calliess in: ders./Ruffert (Hrsg.) EUV/AEUV, 4. Aufl. 2011, Art. 170 Rn. 25. Zur Voraussetzung marktbezogener Tätigkeiten St. Wernicke in: Grabitz/Hilf/Nettesheim (Hrsg.) Das Recht der Europäischen Union (Stand 50. Ergänzungslieferung 2013), Art. 14 Rn. 26 f., weiter mit Nachweisen zur Genese der Terminologie des „gemeinwirtschaftlichen Dienstes“; A. Gyulai-Schmidt VergabeR 2012, 809 (810 f.). Zur Bedeutung des Protokolls Nr. 26, ABl. 2008 C 115/308 vgl. Ch. Jung in: Calliess/Ruffert (Hrsg.) EUV/AEUV, 4. Aufl. 2011, Art. 14 Rn. 14 ff.

119 Franzius Gewährleistung (Fn. 3), 414 ff., zum „Wettbewerb im Markt“; vgl. zu den Kategorien mit Gegenübersetzung von Vergaberecht und Netzregulierung am Beispiel der Wasserversorgung J. Masing VerwArchiv 95 (2004), 151 (156 ff.). Die Anerkennung der Dienste von allgemeinem wirtschaftlichem Interesse (Art. 14, 106 Abs. 2 AEUV) steht sinnbildlich dafür, dass Marktgeschehen Vorleistungen erfordert, die von der öffentlichen Hand, eigenen Netzunternehmen oder eben auch von Wettbewerbern der Dienstebene erbracht werden können. Beispielhaft zeigte zuletzt die Debatte um die Wasserversorgung, dass prinzipielle Ableitungen aus den europäischen Rechtsvorgaben den Filter des politischen Kompromisses durchlaufen, vgl. dazu Achtes Gesetz zur Änderung des Gesetzes gegen Wettbewerbsbeschränkungen vom 26. Juni 2013, BGBl. I 1738 Art. 1 Nr. 12 (§§ 31-31b), vgl. Kommission: Vorschlag für Richtlinie des Europäischen Parlaments und des Rates über die Konzessionsvergabe (sog. Konzessionsrichtlinie) vom 20.12.2011, KOM (2011), 897 endg.

120 Auf der Ebene des sekundären Unionsrecht werden weitreichende Entflechtungsziele insbesondere im Energiebereich verfolgt: vgl. die Richtlinie 2009/72/EG des Europäischen Parlaments und des Rates vom 13. Juli 2009, ABl. L 211/55. Aus dem jüngeren Schrifttum A.-B. Kaiser/Th. Wischmeyer VerwArch 101 (2010), 34 ff.; für einen frühen Überblick über die Sektoren E. Staebe IR 2006, 204 ff., 222 ff.; J. Masing Soll das Recht der Regulierungsverwaltung übergreifend geregelt werden?, Gutachten D zum 66. DJT, 2006, D 114 ff. Zur aktuellen Lage im Eisenbahnrecht $U$. Ehricke N\&R 2012, 222 (223 f.); zu den europarechtlichen Vorgaben Ch. Lerche N\&R 2013, 27 (29); umfassend $P$. Hafner Entflechtung und Wettbewerb im Eisenbahnwesen, 2011, insb. 53 ff. 
der Infrastrukturen, stärker in den Blick nehmen. Zerfaserte Straßenbeläge, gesperrte Bahntrassen und Autobahnbrücken, das Problem des Rückbaus vor allem im Atomrecht, aber auch die bessere Nutzung der Windenergieanlagen und die Aufwertung von Telekommunikationsleitungen stehen in gewisser Weise für die doppelte Dimension des Themas Infrastruktur überhaupt: Der erreichte Standard ist bereits hochkomplex, wartungsintensiv und im Zeitablauf störanfällig; und darüber hinaus ist eine stetige Verbesserung der Nutzung technologisch, ökonomisch wie ökologisch reizvoll, ja oftmals geboten.

Das Recht widmet sich der Pflege der Infrastruktur nur ausschnittsweise und vor allem ungleichmäßig. ${ }^{121}$ Diese Unterbelichtung lässt sich in erster Linie als Folgefehler der Ausrichtung auf abschließende Entscheidungen erklären. In der durchscheinenden Logik des Gewerberechts ${ }^{122}$ ist die spätere Behandlung der Einrichtung Sache und Risiko des Eigentümers. ${ }^{123}$ Damit wird aber die Vielschichtigkeit öffentlicher Infrastrukturen

121 Zum Gesamtrahmen im Bereich der Eisenbahn M. Fehling Das Recht der Eisenbahnregulierung, in: Lüdemann (Hrsg.) Telekommunikation, Energie, Eisenbahn, 2008, 118 (122 ff.), vgl. insb. $\S \S 4$ Abs. 3, 4a, 11 AEG. Zur Neufassung der Eisenbahnregulierung BT-Drs. 17/12726 von 2013, vgl. dazu Stellungnahme T. Beckers, der für eine „effiziente Erhaltungsstrategie“ plädiert, da bisher ,auf Verschleiß“ gefahren werde, zu finden, Drs. 17/15563 (Stellungnahmen), 59 ff.; zum Hintergrund U. Ehricke N\&R 2012, 222 (225 ff.). Für den Hochwasserschutz wurde mit der Begründung der Gefahrenabwehr zuletzt eine Verkürzung der üblichen Genehmigungsanforderungen gefordert, vgl. bereits Fn. 6. Dagegen konzentrieren sich die neuartigen Regulierungsgebiete eher auf Gewährleistungspflichten in Bezug auf die Dienste, bei denen die Vorhaltung der Infrastruktur selbst nur als Reflex beachtet wird. Vgl. zu den entsprechenden Pflichten der Betreiber von Übertragungsnetzen K. Bourwieg in: Britz/Hellermann/Hermes (Hrsg.) EnWG, 2. Aufl. 2010, § 12 Rn. 6 ff.; vgl. als Beispiel für gesetzgeberische Detailregelung den neuen $\S 12$ Abs. 3a EnWG. Anders gefasst ist die Pflege etwa Teil der „nachhaltigen“ Energieversorgung, dazu i E J. Gundel Nachhaltigkeit und Energieversorgung, in: Kahl (Hrsg.) Nachhaltigkeit als Verbundbegriff, 2008, 443 (445 für eine engere, nämlich umweltbezogene Begriffsverwendung). Für den Bereich der Wasserkraftnutzung in Bezug auf vorhandene Anlagen nach $\S 35$ WHG M. Reinhardt NVwZ 2011, 1089 (1092 f.). Für die Rechtslage im Telekommunikationsrecht 2012 C. Franzius N\&R 2012, 126 (136 ff.). Eine echte „Investitionsregulierung“ findet insgesamt nicht statt, zu dieser Formel J. Kühling DVB1. 2013, 1093 (1100). Zum Ansatz einer ,reflexiven Steuerung durch Investitionsplanungspflichten“ J. P. Schneider Infrastrukturausbau als Aufgabe des Regulierungsrechts, in: Fehling/Grewlich (Hrsg.) Struktur und Wandel des Verwaltungsrechts, 2011, 69 (79 ff.).

122 Vgl. Mann Großvorhaben (Fn. 4), 556 ff.; in der Grundanschauung wohl auch Waechter Großvorhaben (Fn. 4), $518 \mathrm{f}$.

123 Der Gesetzgeber und das Bundesverwaltungsgericht mit seiner Rechtsprechung zur Einschränkung späterer Auflagen haben bisher das ihre dazu getan, dass das statische Modell der Anlagengenehmigung den besonderen Charakter der öffentlichen Infrastrukturen vernachlässigt, vgl. BVerwGE 112, 221 (224 ff.). Zur grundrechtlichen 
in gemischten Realisierungsmodellen verfehlt. ${ }^{124}$ Ansatzpunkt für eine angemessene Weiterentwicklung können hier die Modelle liefern, die in der Zugangs- und Preisregulierung oder auch im Anlagenzulassungsrecht entwickelt worden sind: Denn hier ist der Dauercharakter der entsprechenden Verwaltungsaufgabe bereits früh erkannt worden. ${ }^{125}$ Wenn Genehmigungen nicht als Abschluss eines Verwaltungsverfahrens, sondern als Etappe der Dauerverwaltung Infrastruktur begriffen werden, ist auch die nachlaufende Aktualisierung der entsprechenden Rechtsverhältnisse verbessert möglich. ${ }^{126}$

Lage $P . \quad M$. Huber Überwachung, in: Hoffmann-Riem/Schmidt-Aßmann/Voßkuhle (Hrsg.) Grundlagen des Verwaltungsrechts, Band III, 2. Aufl. 2013, § 45, insb. Rn. 7 f. Vgl. zum Instrument des Heimfalls am Beispiel des Wasserrechts $R$. Breuer Probleme des wasserrechtlichen Heimfalls, 2009, insb. $47 \mathrm{ff}$.

${ }^{124}$ So ist etwa die Schließung öffentlicher Einrichtungen „,vor Ort“" von vornherein eine durchgängig (kommunal-) politisch verantwortete Angelegenheit.

${ }^{125} \mathrm{Vgl}$. für den „traditionellen Zukunftsbezug des Verwaltungsrechts“ allgemein Appel Staatliche Vorsorge (Fn. 85), 129, zu den Instrumenten insb. 134 ff. Die begleitende Sorge ist insbesondere im Umweltrecht entwickelt worden und hat sich in $\S 5$ BImSchG manifestiert.

${ }^{126}$ Zum Topos der Marktbegleitung M. Eifert Regulierungsstrategien, in: Hoffmann-Riem/Schmidt-Aßmann/Voßkuhle (Hrsg.) Grundlagen des Verwaltungsrechts, Band I, 2. Aufl. 2012, 19 Rn. 127. Ein wichtiges Element der (weiteren) Begleitung privater Akteure ist die Wissensgenerierung, zu den entsprechenden regulierungsrechtlichen Instrumenten vgl. kritisch K. F. Gärditz DVBl. 2009, 69 ff. Das allgemeine Verwaltungsverfahrensrecht sowie die Fachgesetze kennen selbstverständlich Reaktionsmöglichkeiten auf nachträgliche Veränderungen. Auflagenvorbehalte, Erweiterungsgenehmigungen oder nachträgliche Anordnungen (zB § $17 \mathrm{BImSchG)} \mathrm{sind} \mathrm{jedoch} \mathrm{nicht}$ im Sinne der Dauerverwaltung auf das Ziel der qualitätsbezogenen Pflege und Erhaltung von Infrastrukturen ausgerichtet, sondern bezwecken die Vermeidung bzw. den Ausgleich von Nachteilen Dritter (Nachbarn etc.) und die Einhaltung ordnungsrechtlicher Pflichten. Das Planfeststellungsrecht ermöglicht es, im Planfeststellungsbeschluss neben Schutzauflagen zugunsten betroffener Dritter in gewissem Umfang auch Vorkehrungen ,zum Wohl der Allgemeinheit“ aufzuerlegen (vgl. § 74 Abs. 2 S. 2 VwVfG). Darunter werden allerdings vorrangig Aspekte der Verkehrssicherheit verstanden. Inwiefern Betriebsregelungen Gegenstand der Planfeststellung sein können, wird in den einzelnen Fachgesetzen unterschiedlich geregelt und ist im Einzelnen umstritten; vgl. dazu W. Blümel VerwArch 83 (1992), 146 ff. Gem. § 9 Abs. 2 LuftVG sind im Planfeststellungsbeschluss dem Unternehmer die Errichtung und Unterhaltung der Anlagen aufzuerlegen, die für das öffentliche Wohl oder zur Sicherung der Benutzung der benachbarten Grundstücke gegen Gefahren oder Nachteile notwendig sind. Zur Ökonomisierung rechtlicher Technikanforderungen am Beispiel des Wasserrechts E. Gawel DÖV 2012, 298 (300 ff.). Zu den erweiterten Regelungen des TKG 2012 B. Kindl M. Schramm N\&R 2012, 140 (141 ff.); M. Freund/T.-L. Bary NVwZ 2012, 1504 ff.; vgl. zur Inanspruchnahme öffentlicher Wege U. Rathgeb NVwZ 2012, 270 (271 ff.). Zur Methodik der rechtsaktbezogenen Arbeitsweise I. Appel und M. Eifert Das Verwaltungsrecht zwischen klassischem dogmatischen Verständnis und steuerungswissen- 


\section{Zusammenfassung: Infrastrukturrecht als Verbundrecht- Infrastrukturverwaltung als Dauerverwaltung}

Zusammenfassend lässt sich erkennen, dass ein zukunftsfähiges Recht der öffentlichen Infrastrukturen die geschilderten Phasen als Verbund begreifen muss. Der teilweise anzutreffende Abbruch rechtlicher Betreuung wird der Rolle der öffentlichen Infrastruktur nicht gerecht. Soweit private Akteure beteiligt werden, verbindet sich mit der weitgehenden Offenheit für solche Kooperationen die klare Auskunft, dass die Beteiligung an öffentlichen Aufgaben ggfs. besondere Rechte, in jedem Fall aber erhöhte Pflichtenbindungen mit sich bringt. Freiheitssicherung kann dort angesichts der multipolaren Rechtsverhältnisse nicht durch möglichst weitgehende unternehmerische Privatautonomie realisiert werden, sie benötigt vielmehr invers ausgerichtete Sicherungen, die sich als Verfahrens-, Beteiligungs- und Gestaltungsansprüche beschreiben lassen. ${ }^{127}$ Hier werden zukünftig weiter zunehmend komplexe Grundrechtslagen entstehen, bei denen sich Berechtigung und gesetzlich geregelte Verpflichtung der beteiligten Unternehmen verbinden. ${ }^{128}$

Gegen die vielfach vertretene Ansicht, die in den Netzwirtschaften etablierten neuen Arrangements hätten sich zu einem Normalfall des Infra-

schaftlichem Anspruch, VVDStRL 67 (2008), 226 (252 ff.) bzw. 286 (289 ff.). Zu Kategorien eines pflegenden Gewährleistungsverwaltungsrechts bereits Voßkuhle (Fn. 3), $310 \mathrm{ff}$.

127 Zur Spannung von individueller und kollektiver Freiheitsentfaltung für diesen Zusammenhang mit individuellrechtlichem Akzent J. Kersten Teilverfasste Wirtschaft, in: Vesting/Korioth (Hrsg.) Der Eigenwert des Verfassungsrechts, 2011, 135 (145 ff.); zurückhaltender $O$. Lepsius Der Eigenwert der Verfassung im Wirtschaftsrecht, ebd., 149 (167 ff.).

${ }^{128}$ Das gilt zunächst für vom Staat beherrschte gemischtwirtschaftliche Unternehmen, vgl. BVerfGE 128, 226 - Fraport, dort nochmals erweiternd 249 f. als obiter dictum auch für materiell private Unternehmen. Umfassende Aufbereitung bei J. P. Schaefer Der Staat 51 (2012), 250 ff.; vgl. für die Etappen der Debatte Storr Staat (Fn. 74) 187 ff.; U. Battis/J. Kersten WuV 2005, 493 (495 ff.); C. Bumke Die Verwaltung 41 (2008), 227 (238); J. Wieland Die Verwaltung 43 (2010), 83 (88); Hellermann Schutz der Verbraucher (Fn. 72), 389 f.; H. Wißmann Verfassungsrechtliche Vorgaben der Verwaltungsorganisation, in: Hoffmann-Riem/Schmidt-Aßmann/Voßkuhle (Hrsg.) Grundlagen des Verwaltungsrechts, Band I, 2. Aufl. 2012, § 15 Rn. 4a; kritisch P. M. Huber FS Stober, 2008, 547 (551 ff.); F. Schorkopf JZ 2008, 20 (23 f.). Zur Analyse der „Kommune als Konzern“ $J$. Oebbecke VBIBW 2010, $1 \mathrm{ff}$. Wichtig ist die grundgesetzlich gebotene Unterscheidung, dass die Grundrechtsverpflichtung materiell privater Akteure nach wie vor nur als gesetzliche Pflicht in Betracht kommt, sich also an dem formalen Modell des rechtsstaatlichen Verteilungsprinzips nichts ändert, vgl. J. Masing Der Rechtsstatus des Einzelnen im Verwaltungsrecht, in: Hoffmann-Riem/Schmidt-Aßmann/Voßkuhle (Hrsg.) Grundlagen des Verwaltungsrechts, Band I, 2. Aufl. 2012, $\S 7$ Rn. $142 \mathrm{ff}$. 
strukturrechts entwickelt, der als abstrakter Maßstab jede Abweichung rechtfertigungsbedürftig stellt, ${ }^{129}$ ist allerdings daran festzuhalten, dass das Infrastrukturrecht typischerweise gemischte Regelungsmodelle etabliert, die in jedem Sektor einem spezifischen, nicht zuletzt entwicklungsgeschichtlich geprägten Pfad folgen. Es gibt insoweit keinen prinzipiellen infrastrukturrechtlichen Maßstab. ${ }^{130}$

Für die öffentliche Hand gilt, dass öffentliche Infrastrukturen gerade für den Fall einer Einbeziehung privater Akteure einen Regelfall der ,,vorgängig"-begleitenden Dauerverwaltung bilden. ${ }^{131}$ Das hergebrachte Verständnis des Verwaltungsakts oder des Verwaltungsvertrags setzt Annahmen hinsichtlich Personal, Institutionen, ja insgesamt rechtskultureller Grundregeln voraus, die in vielen Bereichen des Infrastrukturrechts planmäßig aufgelöst worden sind. ${ }^{132}$ In der Konsequenz folgt daraus, ${ }^{133}$ dass Handlungsformen und umgebendes ,setting“ im Verwaltungsrecht neu zugeordnet werden müssen. ${ }^{134}$ Aufgabe des allgemeinen Verwaltungs-

129 So in der Gegenübersetzung von TKG und Energierecht zuletzt J. Kühling DVB1. 2013, 1093 (1101), mit klarem Votum für die stärker marktwirtschaftliche Ausrichtung des TKG und kritisch zur Verdrängung der „Kraft des Wettbewerbs“; gegenläufig in Bezug auf das TKG C. Franzius N\&R 2012, 126 (132).

130 Zum Verhältnis unterschiedlicher Regulierungsstrategien M. Eifert Regulierungsstrategien, in: Hoffmann-Riem/Schmidt-Aßmann/Voßkuhle (Hrsg.) Grundlagen des Verwaltungsrechts, Band I, 2. Aufl. 2012, § 19, insb. Rn. 153 ff. Zu den Möglichkeiten und Grenzen von Folgerichtigkeit und Konsistenz allgemein konzeptionell zusammenführend L. Michael Formen- und Instrumentenmix, in: Hoffmann-Riem/SchmidtAßmann/Voßkuhle (Hrsg.) Grundlagen des Verwaltungsrechts, Band II, 2. Aufl. 2012, $\S 41$, insb. 77 ff. Die institutionelle Teilzusammenfassung im Infrastrukturbereich hat an diesem Befund nichts geändert: Schon unter dem Dach der Bundesnetzagentur, die „für Elektrizität, Gas, Telekommunikation, Post und Eisenbahnen“ zuständig ist (Gesetz über die Bundesnetzagentur für Elektrizität, Gas, Telekommunikation, Post und Eisenbahnen (BEGTPG) v. 07.07.2005, BGB1. I 1970) werden bekanntlich weiterhin durchaus verschiedene Regelungsmodelle zur Anwendung gebracht.

131 Zum Topos der ,,vorgängigen Verhandlung“ im Königreich Hannover als Gegenmodell zur preußischen Vollzugsverwaltung und die damit verbundenen verfahrensrechtlichen Implikationen $H$. Faber DVB1. 1985, 715 (719).

132 Treffend insoweit der Hinweis von $R$. Wulfhorst DÖV 2011, 581 (586), auch nach geltendem Recht wäre niemand gehindert, eine vorbildliche Bürgerbeteiligung durchzuführen.

133 R. Wahl Die Aufgabenabhängigkeit von Verwaltung und Verwaltungsrecht, in: Hoffmann-Riem/Schmidt-Aßmann/Schuppert (Hrsg.) Reform des Allgemeinen Verwaltungsrechts, 1993, 177 (213); Schmidt-Aßmann Ordnungsidee (Fn. 3), Rn. 1/9 ff., insb. 16.

134 Zur Betrachtung aus ,mittlerer Abstraktionshöhe“ vgl. etwa F. Schoch Der Verwaltungsakt zwischen Stabilität und Flexibilität, in: Hoffmann-Riem/Schmidt-Aßmann (Hrsg.) Innovation und Flexibilität des Verwaltungshandelns, 1994, 199 (insb. 212 f.). $\mathrm{Zu}$ Handlungsformen als Ordnungsmustern des Verwaltungsrechts $H$. Wißmann/ 
rechts ist es hier insbesondere, das begrenzte Wirkungsfeld der rechtsformorientierten Vorgehensweise im Bereich der öffentlichen Infrastrukturen aufzunehmen und zu ergänzen: ${ }^{135}$ Dort, wo an die Grundentscheidung für eine öffentliche Infrastruktur eine nicht öffentlich-rechtliche Handlungslogik anschließt (also bei der Einbeziehung privater Akteure), muss die weitere Begleitung solcher Projekte als reguläre Aufgabe angenommen werden, die sich typischerweise nicht an den pathologieorientierten Grundmustern des Ordnungsrechts orientieren kann. ${ }^{136}$

P. Lange Handlungsformen im europäischen Verwaltungsrecht, in: Leible/Terhechte (Hrsg.) Rechtsschutz und Verfahrensrecht (EnzEur Band 3), i. E., § 31 Rn. 7 ff.; C. Franzius Entwicklungsstufen der Verwaltungsrechtswissenschaft, in: HoffmannRiem/Schmidt-Aßmann/Voßkuhle (Hrsg.) Grundlagen des Verwaltungsrechts, Band I, 2. Aufl. 2012, § 4, insb. Rn. 37 ff. Zur wechselseitigen Beeinflussung allgemeiner Handlungsformen und spezifischer Lagen im Infrastrukturrecht J. Saurer DVBl. 2012, 1082 (1082 f.). Stellvertretend für die Skeptiker zuletzt K. F. Gärditz Die Rolle des parlamentarischen Gesetzgebers im Regulierungsrecht - ein Werkstattbericht, in: Kurth/ Schmoeckel (Hrsg.) Regulierung im Telekommunikationssektor, 2012, 67 („Entformalisierung von Herrschaft“ durch „Entparlamentarisierung“ und ,administrative Komplexitätssteigerung"). Das Recht der Infrastrukturen wird mit anderen Worten zugleich überschätzt und unterschätzt, wenn es neben dem Recht der Eingriffs- und Leistungsverwaltung als eigenständiger Typus eingeordnet wird, der durch bestimmte Handlungsformen gekennzeichnet ist, so namentlich Faber Verwaltungsrecht (Fn. 55), 347 ff., der (zu Recht) die Multilateralität und (zu Unrecht) den Ausschluss konkreter rechtlicher Entscheidungen als Merkmal der Infrastrukturverwaltung ansieht (348).

${ }_{135}$ Entsprechend zum Verhältnis von Allgemeinem Verwaltungsrecht und Fachverwaltungsrecht Schmidt-Aßmann Ordnungsidee (Fn. 3), Rn. 1/9 ff., 3/1 ff.; zuletzt in der Übersicht M. Burgi Rechtsregime, in: Hoffmann-Riem/Schmidt-Aßmann/Voßkuhle (Hrsg.) Grundlagen des Verwaltungsrechts, Band I, 2. Aufl. 2012, § 18 Rn. 96 ff.

136 Vgl. zur „Veränderung in der Zeit“ grundlegend Ch. Bumke Relative Rechtswidrigkeit, 2004, 163 ff., insb. zum Verwaltungsakt 172 ff. Andeutungsweise aus Sicht der Technik zur „,integrativen Planung von Infrastrukturmaßnahmen“ St. Trülzsch/ T. Schmidt IR 2012, 325 (326). Zur „Überwachung als Daueraufgabe“ P. M. Huber Überwachung, in: Hoffmann-Riem/Schmidt-Aßmann/Voßkuhle (Hrsg.) Grundlagen des Verwaltungsrechts, Band III, 2. Aufl. 2013, § 45 Rn. 109 ff., vgl. auch Rn. 39 ff. Zum Aspekt der Verwaltungslegitimation durch und in Kooperation mit Privaten $H$. $-H$. Trute Die demokratische Legitimation der Verwaltung, in: Hoffmann-Riem/SchmidtAßmann/Voßkuhle (Hrsg.) Grundlagen des Verwaltungsrechts, Band I, 2. Aufl. 2012, $\S 6$ Rn. 97 ff.; zur Kooperation als „Grundmodus der Aufgabenwahrnehmung“ H. Schulze-Fielitz Grundmodi der Aufgabenwahrnehmung, in: Hoffmann-Riem/ Schmidt-Aßmann/Voßkuhle (Hrsg.) Grundlagen des Verwaltungsrechts, Band I, 2. Aufl. 2012, § 12 Rn. 64 ff., insb. 73 ff. 


\section{Insbesondere: Das Verhältnis von Verwaltungsarbeit und Gesetz im Infrastrukturrecht}

\section{Infrastrukturrecht als Recht politischer Gestaltung}

Die Formel von der Infrastrukturverwaltung als Daueraufgabe hat institutionelle, verfahrensmäßige und materielle Auswirkungen. Eine besondere Herausforderung, die abschließend näher betrachtet werden soll, stellt die Einsicht in den notwendig politischen Gehalt von Infrastrukturentscheidungen dar. Wenn über öffentliche Infrastrukturen im Regelfall weder im „ob" noch im „wie“ verbindliche Vorfestlegungen getroffen sind, endet freilich nicht im Anschützschen Sinn die Aufgabe des Öffentlichen Rechts: Ganz im Gegenteil soll es nach der Anschlussfähigkeit sektoraler Teillösungen fragen (und der Behauptung sachlicher Besonderheit einen Grundzweifel entgegensetzen), auf die innere Konsistenz im Sinn einer relativen Pfadabhängigkeit bestehen (man kann nicht privatisieren und dann wie im Anstaltsbetrieb führen wollen), die verfassungsrechtliche Gesamtsaldierung von Regelungen thematisieren (also bei prozeduralen Entscheidungsabläufen die Banalisierung von Verfahrensfehlern skandalisieren), insgesamt also auf Klugheits- wie auf Richtigkeitsentscheidungen drängen. ${ }^{137}$

Als konkrete rechtsdogmatische Weiterentwicklung ist die bisherige Abfolge von Verwaltungsarbeit und Gesetz zu überprüfen. Die hier entfaltete Dauerperspektive wird anders gesagt unterschätzt, wenn nur auf Verwaltungsabläufe geblickt wird. Ein leistungsfähiges Infrastrukturrecht kann und sollte - so die abschließende Perspektive - die gesetzliche Entscheidung über konkrete Infrastrukturvorhaben als Instrument in neuer Weise für sich (wieder-138) entdecken und damit besser abgeschichtete und legitimierte Infrastrukturentscheidungen möglich machen.

${ }_{137}$ Vgl. A. Voßkuhle Die Verwaltung 32 (1999), 545 ff. Für den Wandel der Herausforderungen in Bezug auf die Verwaltung $S$. Baer Verwaltungsaufgaben, in: HoffmannRiem/Schmidt-Aßmann/Voßkuhle (Hrsg.) Grundlagen des Verwaltungsrechts, Band I, 2. Aufl. 2012, § 11 Rn. 15 ff., 23 ff.; H. Kube Die Verwaltung 41 (2008), 1 (26: „Verfassungsrechtliche Begleitung formeninnovativer Aufgabenerfüllung"). In diesem Sinn ist auch die bei $W$. Erbguth DÖV 2012, 821 (825 ff.) geforderte „Neutralitätssicherung“ der staatlichen Raumordnung zu unterstützen.

138 (Überwiegend kritische) Überlegungen zum Einzelfallgesetz insbesondere für Großvorhaben bereits bei $V$. Henle UPR 1982, 215 ff., 253 ff.; P. Badura FS H. Huber, 1981, 15 (23 f.); M. Ronellenfitsch DÖV 1991, 771 (776 ff.); P. Kunig Jura 1993, 308 ff.; F. Ossenbühl FS Hoppe, 2000, 183 ff.; vorsichtig positiv zuletzt Th. Groß DÖV 2011, 510 (514). Beispiele für vor- und nachkonstitutionelle Investitionsmaßnahmengesetze bei Ch. Schneller Objektbezogene Legalplanung, 1999, 24 ff. 
2. Infrastrukturentscheidungen in der verfassungsrechtlichen Funktionengliederung

\section{a) Gewaltenteilung und Einzelfallgesetz}

Freilich stehen einem solchen Ansatz scheinbar das spezielle Verbot des Einzelfallgesetzes wie der allgemeine Gedanke der Gewaltenteilung entgegen. Insbesondere das imperfekte Verbot des Art. 19 Abs. 1 Satz 1 GG zeugt von der Skepsis, dass ein staatlicher Machtblock ,durchentscheidet" und die verfassungsstaatliche Mäßigung außer Kraft setzt, die durch die Trennung von Gesetzgebung und Gesetzesanwendung gesichert werden soll. ${ }^{139}$

Dennoch weist bereits die fehlende Wirkungsgeschichte der Vorschrift $^{140}$ darauf hin, dass es sich bei ihr um eine konstitutionalistische

139 Vgl. allgemein BVerfGE 22, 106 (111) - Steuerausschüsse; allgemeiner BVerfGE 3, 225 (247) - Gleichberechtigung; 95, 1 (15) - Südumfahrung Stendal. In verfassungsrechtlichen Grundkategorien: „Rechtsstaat und Demokratie sind, soweit sie praktisch werden wollen, auf Gewaltenteilung verwiesen." - R. Poscher Funktionenordnung des Grundgesetzes, in: Hoffmann-Riem/Schmidt-Aßmann/Voßkuhle (Hrsg.) Grundlagen des Verwaltungsrechts, Band I, 2. Aufl. 2012, § 8 Rn. 19; vgl. weiter Ch. Möllers AöR 132 (2007), 493 (496 f.); M. Cornils Gewaltenteilung, in: Depenheuer/Grabenwarter (Hrsg.) Verfassungstheorie, 2010, § 20 Rn. 8 ff., 27 ff. Zu beachten ist, dass das Gesetzgebungsverfahren auch isoliert ein spezifisches Rationalitätsprogramm enthält, vgl. dazu F. Reimer Parlamentsgesetz, in: Hoffmann-Riem/Schmidt-Aßmann/Voßkuhle (Hrsg.) Grundlagen des Verwaltungsrechts, Band I, 2. Aufl. 2012, § 9 Rn. 5 ff.

${ }_{140}$ Bekanntlich hat das BVerfG auch in mehr oder weniger offensichtlichen Fällen Parlamentsgesetze in keinem Fall an Art. 19 Abs. 1 S. 1 GG scheitern lassen, vgl. einschlägig vor allem BVerfGE 7, 129 (151 ff.) - lex Schörner (Anlassgesetzgebung); 10, 234 (244 f.) - „Platow-Komplex“ (verdecktes Individualgesetz); 13, 225 (228 f.) - Bahnhofsapotheke (faktisches Einzelfallgesetz); 24, 367 (395 ff.) - Hamburger Deichordnung (Ausschluss für Art. 14 GG); 25, 371 - Montanmitbestimmung I (LS 1 f.: „1. Der Begriff des Maßnahmegesetzes ist verfassungsrechtlich irrelevant. 2. Einzelfallgesetze sind als solche nach dem Grundgesetz nicht schlechthin unzulässig. (...)"), insb. $398 \mathrm{f}$. („Auch die gesetzliche Regelung eines einzelnen Falls kann erforderlich sein. Dies gilt vor allem im Bereich der Wirtschafts- und Sozialordnung"), hier 399 auch besonders deutlich die dogmatische Argumentation zu den ,speziellen“ Vorbehalten; 85, 360 (374) - Akademie der Wissenschaften der DDR (Regelung eines singulären Sachverhalts); 95, 1 (15 ff.) - Südumfahrung Stendal (gesetzliche Einzelentscheidung bei ,guten Gründen“); vgl. zuletzt 99, 367 (400 f.) - Montanmitbestimmung II; 121, 30 (49) - Parteirundfunk. In der Literatur sind als Hauptfragen die Verwirklichung der Gewaltenteilung, v.a. aber der qualifizierte Gleichheitsschutz identifiziert worden, vgl. dazu differenziert A. von Arnauld Die Freiheitsrechte und ihre Schranken, 1999, 179 ff.; übersichtliche Darstellung bei P. M. Huber in: v. Mangoldt/Klein/Starck (Hrsg.) Kommentar zum Grundgesetz, 6. Aufl. 2010, Art. 19 Rn. 11 ff.; ausführlich J. Lege Verbot des Einzelfallgesetzes, in: Merten/Papier (Hrsg.) Handbuch der Grundrechte, Band 3, 2009, $\S 66$ Rn. 17 ff., 31 ff. Zur Entstehung der Vorschrift G. Kirchhof Die Allgemeinheit des 
Verspätung handelt, die die tieferliegende Struktur des Grundgesetzes verfehlt: ${ }^{141}$ Das Verbot des Einzelfallgesetzes geht gedanklich davon aus, dass Parlament und Regierung Gegenspieler sind, und dass ihre „Einigung" durch Gesetz und Verwaltungsakt alle Legitimationsbedürfnisse hinreichend befriedigt und zugleich den grundrechtlichen Schutz des Einzelnen absichert; das ist nun aber erkennbar in der parlamentarischen Regierungsform nicht sicher gegeben. ${ }^{142}$

Gesetzes, 2009, 196 ff., mit Forderungen zur weiteren Entfaltung 207 ff. Knapper rechtsvergleichender Überblick bei $H$. Dreier in: ders. (Hrsg.) GG-Kommentar, 3. Aufl. 2013, Art. 19 Abs. 1 Rn. 8. Vgl. zur Praxis in der Schweiz Fn. 148.

${ }^{141}$ Dem liegt eine übersteigerte und letztlich zugleich geringschätzende Einordnung des allgemeinen Gesetzes zugrunde, dem die Kraft der „Maßnahme“ abgesprochen wird. Diese funktionelle Scheinidealisierung angeblicher Rechtsstaatsprinzipien findet sich pointiert bei C. Schmitt Verfassungslehre, 1928, 138 ff. (142: der generelle Charakter einer Rechtsnorm als „der letzte Rest der ideellen Grundlage des bürgerlichen Rechtsstaats überhaupt"), sowie dann (programmatisch aufgenommen und zugleich als Teil einer Verfallsgeschichte praktisch relativiert) bei E. Forsthoff GS W. Jellinek, 1955, 221 (221 f., 229); sehr allgemein auch Ch. Möllers Gewaltengliederung, 2006, 105 ff.; dagegen schon P. Laband Deutsches Reichsstaatsrecht I, 1907, 106 f. (Allgemeinheit als „Naturale, nicht ein Essentiale des Gesetzesbegriffs"); H. Heller Der Begriff des Gesetzes in der Reichsverfassung, VVDStRL 4 (1927), 98 (107 ff., 121); zur Einordnung O. Lepsius Der Hüter der Verfassung - demokratietheoretisch betrachtet, in: Beaud/ Pasquino (Hrsg.) La controverse sur „le gardien de la Constitution“ et la justice constitutionnelle / Der Weimarer Streit um den Hüter der Verfassung und die Verfassungsgerichtsbarkeit, 2007, 103 (110 ff.). Vgl. zur Debatte unter dem Grundgesetz vor allem K. Zeidler Maßnahmegesetz und „klassisches“ Gesetz, 1961, insb. 143 f.; U. Scheuner FS H. Huber, 1981, 127 (132); zusammenfassend Schneller Legalplanung (Fn. 138), 26, 54 ff.; für die Gegenwart W. Krebs in: v. Münch/Kunig (Hrsg.) GG, 6. Aufl. 2012, Art. 19 Rn. 6 ff. Einschlägiger Überblick zur Debatte bei J. Bücker Die Zulässigkeit von Individualgesetzen nach dem Grundgesetz, 1966, 8 ff. Die gelegentlich behauptete Abwehr eines Parlamentsabsolutismus angesichts der Erfahrungen des Nationalsozialismus lässt sich rechtsempirisch kaum halten, vgl. H. Dreier in: ders. (Hrsg.) GG-Kommentar, Band 1, 3. Aufl. 2013, Art. 19 Abs. 1 Rn. 13 ff. Vgl. gegen die These von „Degenerationsentscheidungen“ auch M. Cornils Gewaltenteilung, in: Depenheuer/Grabenwarter (Hrsg.) Verfassungstheorie, 2010, $§ 20$ Rn. 33 ff., s. a. Rn. 43 zur Kritik des legitimationstheoretisch-materiellen Ansatzes von Möllers.

${ }^{142}$ Vgl. zur präkonstitutionellen Idee der Machtteilhabe durch Gewaltenteilung bei Montesquieu M. Cornils Gewaltenteilung, in: Depenheuer/Grabenwarter (Hrsg.) Verfassungstheorie, 2010, $\$ 20$ Rn. 9. Zur Rezeption der Gewaltenteilung im Frühkonstitutionalismus St. Korioth Der Staat 37 (1998), 27 (38 ff.); M. Racky Die Diskussion über Gewaltenteilung und Gewaltentrennung im Vormärz, 2005, insb. 55 ff., 119 ff. In der Situation der „Parlamentsregierung“ kann typischerweise eine Einigung zwischen Parlamentsmehrheit und gubernativ geführter Verwaltung vorausgesetzt werden - und daher versagt dieses Legitimationsarrangement (zumindest jenseits der hier typischerweise auch nicht angeführten vertikalen Gewaltenteilung gemäß Art. 83 GG). Zu dieser Einsicht im Überblick referierend U. Di Fabio Gewaltenteilung, in: Isensee/Kirchhof 
Der eigentliche Kern der Funktionenordnung des Grundgesetzes wie des europäischen Rechts setzt deswegen abstrakter an:143 Das Verfassungsrecht macht sich die Mäßigung der Staatsgewalt und die Verbindung möglichst hoher Sachkunde mit möglichst breiter Legitimation zum Programm - eine zwingende Abfolge von allgemeinem Gesetz und konkreter Verwaltungsentscheidung ist aber nicht das Synonym dieser Vorgabe, sondern nur eine mögliche Ausprägung; ${ }^{144}$ hieraus erklärt sich, warum die hermetische Formel des Art. 19 Abs. 1 S. 1 GG wirkungslos geblieben ist. ${ }^{145}$

(Hrsg.) Handbuch des Staatsrechts, Band 2, 3. Aufl. 2004, § 27 Rn. 53; R. Poscher Funktionenordnung des Grundgesetzes, Grundlagen des Verwaltungsrechts, Band I, 2. Aufl. 2012, § 8 Rn. 11 ff. Das Bundesverfassungsgericht hat deshalb zuletzt für möglich gehalten, dass außerparlamentarische Beteiligte in einem Gesetzgebungsverfahren zum Beispiel ein Anhörungsrecht haben können, vgl. BVerfG 1 BvR 1501/13 vom 27.6.2013 am Beispiel der Zusammenlegung von Universitäten in Bezug auf betroffene Fakultäten; allgemein F. Reimer Parlamentsgesetz, in: Hoffmann-Riem/SchmidtAßmann/Voßkuhle (Hrsg.) Grundlagen des Verwaltungsrechts, Band I, 2. Aufl. 2012, $\S 9$ Rn. 5 mit Fußnote 21. Vgl. im übrigen BVerfGE 95, 1 (17) - Südumfahrung Stendal: „Dem Grundgesetz kann nicht entnommen werden, daß es von einem Gesetzesbegriff ausgeht, der nur generelle Regelungen zuläßt. Dies bestätigen sowohl Art. 19 Abs. 1 Satz 1 GG, der Einzelfallgesetze nicht generell, sondern nur in seinem Gewährleistungsbereich ausschließt, als auch Art. 14 Abs. 3 Satz 2 GG, der dem Gesetzgeber ausdrücklich die Möglichkeit der Enteignung durch Gesetz eröffnet. Mit der Planung eines einzelnen Vorhabens greift der Gesetzgeber mithin nicht notwendig in die Funktion ein, die die Verfassung der vollziehenden Gewalt oder der Rechtsprechung vorbehalten hat (vgl. BVerfGE 25, 371 [398]). “

143 So dezidiert BVerfGE 95, 1 (15) - Südumfahrung Stendal, unter Zusammenschau von BVerfGE 3, 325 (347) - Gleichberechtigung, und 68, 1 (86) - Atomwaffenstationierung. Für eine funktionale Rekonstruktion der Gewaltenteilung rechtsvergleichend auch Möllers Gewaltengliederung (Fn. 141), 81 ff.; vgl. U. Di Fabio Gewaltenteilung, in: Isensee/Kirchhof (Hrsg.) Handbuch des Staatsrechts, Band 2, 3. Aufl. 2004, § 27 Rn. 50 ff. Zur europäischen Funktionenordnung grundlegend EuGH, Rs. 138/79, Slg. 1980, I-3333; vgl. H. Wißmann/P. Lange Handlungsformen im europäischen Verwaltungsrecht, in: Leible/Terhechte (Hrsg.) Rechtsschutz und Verfahrensrecht (EnzEur Band 3), i. E., § 31 Rn. 4 ff. Vgl. zum klassischen Konzept der Gewaltenteilung zuletzt $M$. Cornils Gewaltenteilung, in: Depenheuer/Grabenwarter (Hrsg.) Verfassungstheorie, 2010, § 20, bereits Rn. 1 zur Spannung zwischen „Idee“ und Verfassungslage; zur entsprechenden Debatte zum Rechtsstaatsprinzip A. v. Arnauld Rechtsstaat, in: Depenheuer/Grabenwarter (Hrsg.) Verfassungstheorie, 2010, § 21 Rn. 1 ff., zur Verschränkung mit dem Demokratieprinzip Rn. 44 ff.

${ }_{144}$ Zur dreifachen Begründung der Gewaltengliederung näher Möllers Gewaltengliederung (Fn. 141), 68 ff. sowie 88 ff.; kritisch zur materiellen Zuschreibung von Funktionen an bestimmte Organe M. Cornils Gewaltenteilung, in: Depenheuer/Grabenwarter (Hrsg.) Verfassungstheorie, 2010, § 20 Rn. 32 ff.

${ }^{145}$ Daher ist verfassungsrechtlich bei der Zuordnung von Staatsfunktionen und Handlungsformen eine summative Betrachtung, die konkrete Festlegungen freilich integriert, einem abstrakten Regel-Ausnahme-Verhältnis vorzuziehen, vgl. dazu im Über- 


\section{b) Vom Einzelfallgesetz zum integrierten Entscheidungsverbund}

Wenn das verfassungsrechtliche Grundszenario also (pragmatisch wie theoretisch begründet) offen ist, sollten die angesprochenen Unfertigkeiten durch einen konsequenten Schritt überwunden werden. Das Ziel wäre ein Entscheidungsverbund, in dem Verwaltungsarbeit und gesetzgeberische Entscheidung funktional adäquat eingesetzt werden: Für öffentliche Infrastrukturen bedeutet dies, dass in einem vorgängigen Verwaltungsverfahren - grundsätzlich nach dem Muster des bisherigen Planfeststellungsverfahrens ${ }^{146}$ - die Materie aufbereitet werden und eine Entscheidung vorbereitet werden kann. ${ }^{147}$ Die Entscheidung selbst wird dann zur Sache des

blick R. Poscher Funktionenordnung des Grundgesetzes, in: Hoffmann-Riem/SchmidtAßmann/Voßkuhle (Hrsg.) Grundlagen des Verwaltungsrechts, Band I, 2. Aufl. 2012, $\S 8$ Rn. 31 ff. Zur entsprechenden Diskussion um die Allgemeinheit des Gesetzes H. Hofmann Das Postulat der Allgemeinheit des Gesetzes, in: Starck (Hrsg.) Die Allgemeinheit des Gesetzes, 1987, 9 (46 ff.). Übersicht zum doppelten Gesetzesbegriff F. Ossenbühl Gesetz und Recht, in: Isensee/Kirchhof (Hrsg.) Handbuch des Staatsrechts, Band 5, 3. Aufl. 2007, $\S 100$ Rn. 5 ff, insb. 9 f. Bei aller Kritik am handwerklichen Standard der Rechtsprechung zu Art. 19 Abs. 1 Satz 1 GG überwiegt das Verständnis für eine „teleologische Reduktion“ (Huber, Hillgruber) der Vorschrift in Bezug auf die hier betroffene Diskussion um Einzelfallgesetze für jeweils singuläre Sachverhalte (in Abgrenzung zu Einzelpersonengesetzen), vgl. insb. H. Dreier in: ders. (Hrsg.) GG-Kommentar, 3. Aufl. 2013, Art. 19 Abs. 1 Rn. 13 ff.; U. Hufeld in: Kahl/Waldhoff/ Walter (Hrsg.) Bonner Kommentar zum Grundgesetz (Neubearbeitung 2012), zusammenfassend Art. 19 Abs. 1 Rn. 176 ff.; B. Remmert in: Maunz/Dürig (Hrsg.) GG-Kommentar (2008), Art. 19 Abs. 1 Rn. 36; Ch. Hillgruber Grundrechtsschranken, in: Isensee/Kirchhof (Hrsg.) Handbuch des Staatsrechts, Band 9, 3. Aufl. 2011, § 201 Rn. 42; F. Ossenbühl FS Hoppe, 2000, 183 (193 f.); strenger P. M. Huber in: v. Mangoldt/Klein/ Starck (Hrsg.) Kommentar zum Grundgesetz, 6. Aufl. 2010, Art. 19, insb. Rn. 22, 49 ff., 59 ff.; J. Lege Verbot des Einzelfallgesetzes, in: Merten/Papier (Hrsg.) Handbuch der Grundrechte, Band 3, 2009, § 66, zusammenfassend Rn. 102 ff.; frühe Kritik v.a. bei H. Krüger DVB1. 1955, 758 ff., 791 ff.; vgl. auch D. Volkmar Allgemeiner Rechtssatz und Einzelakt, Berlin 1962, 212 ff.; Bücker Zulässigkeit von Individualgesetzen (Fn. 141), insb. $41 \mathrm{ff}$., $61 \mathrm{ff}$.

146 Ein entsprechendes Verfahren müsste insbesondere die geeignete Einbeziehung der Öffentlichkeit und der Betroffenen (weiter) sichern. Zugleich wäre es wegen seiner Einbindung in einen nachfolgenden Legitimationszusammenhang nicht gezwungen, die immer weiter aufgetürmten Kaskaden von (Schein-) Beteiligungsritualen fortzuführen, auf die sich die Vorschläge zur „Demokratisierung“ der Planungsentscheidungen konzentrieren, vgl. dazu bereits Fn. 88, 91.

${ }^{147}$ Gesetzesvorbereitende Arbeit ist als Aufgabe der Ministerialbürokratie eine gewohnte Aufgabe der Exekutive, vgl. insbesondere auch zur Einbeziehung ,ausführender" Behörden E. Schmidt-Jortzig/M. Schürmann in: Kahl/Waldhoff/Walter (Hrsg.) Bonner Kommentar zum Grundgesetz (1996), Art. 76 Rn. 213 f.; J. HesselT. Ellwein Das Regierungssystem der Bundesrepublik Deutschland, 10. Aufl. 2012, 408 ff.; kritisch R. Stettner in: Dreier (Hrsg.) GG-Kommentar, Band II, 2. Aufl. 2006, Art. 76 Rn. 20 f. 
Gesetzgebers. ${ }^{148}$ Die Gewichtung der Abwägungsbelange wäre in dieser Perspektive Aufgabe der Verwaltung, ${ }^{149}$ während der parlamentarische Gesetzgeber ähnlich wie bei Referenden am Ende die Entscheidung über feststehende Alternativen zu treffen hat. ${ }^{150}$ Die nachlaufende Verwirklichung des Vorhabens fiele dann wieder an die Infrastrukturverwaltung zurück, wodurch auch der Verbund zu den anschließenden Phasen des Infrastrukturrechts sichergestellt wäre.

Einem solchen Vorschlag stehen von Verfassungswegen weder ein „Verwaltungsvorbehalt"151 (auch nicht in der modernen Gestalt des Bundes-

Zur externen Wissensgenerierung A. Voßkuhle Sachverständige Beratung des Staates, in: Isensee/Kirchhof (Hrsg.) Handbuch des Staates, Band 3, 3. Aufl. 2005, § 43 Rn. 6 ff.; zur europäischen Dimension der „Lernprozesse in gestuften Verbundverfahren“ M. Fehling Eigenwert des Verfahrens (Fn. 101), $313 \mathrm{f}$.

148 Dezidiert anderer Auffassung Waechter Großvorhaben (Fn. 4), 526 ff., der die entsprechende Praxis in der Schweiz kritisiert. Waechter sieht in klassischer Manier die Hauptaufgabe des Parlaments in der abstrakten Festsetzung von Genehmigungsvoraussetzungen, 529; im Ergebnis ähnlich ablehnend K. F. Gärditz GewArch 2011, 273 (278), $R$. Wulfhorst DÖV 2011, 581 (585 f.) jeweils in Bezug auf Volksentscheide. Zuzustimmen ist der Aussage bei F. Ossenbühl FS Hoppe, 2000, 183 (186), dass es sich bei solchen Gesetzen „funktionenrechtlich (um) einen Exekutivakt" handelt. Dennoch ist mit M. Cornils Gewaltenteilung, in: Depenheuer/Grabenwarter (Hrsg.) Verfassungstheorie, 2010, § 20 Rn. 63 ff., darauf abzustellen, dass die formale Handlungsform des Verwaltungsakts dem Parlament nicht zukommt, und gerade aus dieser Erkennbarkeit Rationalität folgen kann.

${ }^{149} \mathrm{Zu}$ den entsprechenden Anforderungen als „Muster“ allgemeiner Überlegungen W. Hoffmann-Riem Eigenständigkeit der Verwaltung, in: Hoffmann-Riem/SchmidtAßmann/Voßkuhle (Hrsg.) Grundlagen des Verwaltungsrechts, Band I, 2. Aufl. 2012, $\S 10$ Rn. 96 ff.

${ }^{150}$ Eine solche Aufteilung berücksichtigt vor allem die quantitativ unterschiedliche Problemlösungskraft. Zieht der Gesetzgeber dagegen Verwaltungsbefugnisse an sich, muss er ggfs. die entsprechenden Verfahrenserfordernisse beachten, vgl. dazu zuletzt in Bezug auf Anhörungsrechte BVerfG 1 BvR 1501/13 vom 27.6.2013. Vgl. für eine Verrechtlichung der Nutzenfeststellung durch das Parlament in Bezug auf den Verkehrswegeplan bereits Th. Groß VerwArchiv 104 (2013), 1 (17).

${ }^{151}$ H. Krüger DVBl. 1955, 791 (791) (Verwaltungsakt als „,Hausgut‘ der Exekutive"), dagegen zuletzt mit der ganz überwiegenden Meinung J. Lege Verbot des Einzelfallgesetzes, in: Merten/Papier (Hrsg.) Handbuch der Grundrechte, Band 3, 2009, § 66 Rn. 17; differenzierend F. Ossenbühl FS Hoppe, 2000, 183 (188), der eine „Normalität“ der Funktionenordnung ausmacht, die nur im Ausnahmefall durchbrochen werden dürfe; für den vorliegenden Zusammenhang Schneller Legalplanung (Fn. 138), 86 ff.; auch die Formel vom „besonderen Sachverstand und der größeren Problemnähe“ der Verwaltung, so statt vieler einschlägig zusammenfassend Schneller Legalplanung (Fn. 138), 213, ist kein materiellrechtlicher Satz. Zur Frage der Einzelfallentscheidungskompetenz des Parlaments Möllers Gewaltengliederung (Fn. 141), 105 ff., 178 ff.; s.a. $R$. Poscher Funktionenordnung des Grundgesetzes, in: Hoffmann-Riem/SchmidtAßmann/Voßkuhle (Hrsg.) Grundlagen des Verwaltungsrechts, Band I, 2. Aufl. 2012, 
netzagenturvorbehalts ${ }^{152}$ ) noch am anderen Ende der Skala eine verabsolutierte Entscheidungsfreiheit des Parlaments ${ }^{153}$ entgegen. Gegenüber solchen parakonstitutionellen Leitmotiven ${ }^{154}$ gibt eine ausgestaltete Verfassung wie das supranational vernetzte Grundgesetz typischerweise andersgeartete Begrenzungen vor. Mit ihnen werden horizontale und vertikale Beteiligung der Staatsfunktionen wie insbesondere der notwendige Rechtsschutz jeweils in einem konkreten Arrangement ausgelotet und definiert. ${ }^{155}$ Richtigerweise streitet die hervorgehobene Legitimation des Par-

$\S 8 \mathrm{Rn} .48 \mathrm{f}$. Tendenziell für ein unbeschränktes Zugriffsrecht auf der Grundlage der Wesentlichkeitslehre einschlägig BVerfGE 95, 1 (16) - Südumfahrung Stendal; G. Zimmer Funktion - Kompetenz - Legitimation, 1979, 263 ff.; kritisch zum Topos F. Reimer Parlamentsgesetz, in: Hoffmann-Riem/Schmidt-Aßmann/Voßkuhle (Hrsg.) Grundlagen des Verwaltungsrechts, Band I, 2. Aufl. 2012, § 9 Rn. 47 f. Allgemein zur Rechtsfigur H. Maurer Der Verwaltungsvorbehalt, VVDStRL 43 (1985), 135 ff.; F. Schnapp ebd., $179 \mathrm{ff}$.

${ }^{152}$ Für den vorliegenden Zusammenhang zum Agenturmodell M. Cornils Gewaltenteilung, in: Depenheuer/Grabenwarter (Hrsg.) Verfassungstheorie, 2010, § 20 Rn. 56. Aus der Flut der Veröffentlichungen J. Masing/G. Marcou (Hrsg.) Unabhängige Regulierungsbehörden, 2010; M. Eifert ZHR 174 (2010), 449 (461 ff.); M. Ludwigs DVBl. 2011, 61 (68 f.); M. Ruffert FS Scheuing, 2011, 399 (410 ff.); C. D. Classen FS Scheuing, 2011, 293 (297 f., 300 ff.); vgl. allgemeiner in Bezug auf europarechtliche Vorgaben E. Frenzel DÖV 2010, 925 ff. Zur Kritik ausführlich K. F. Gärditz AöR 135 (2010), 251 (275 ff.); für die gesamteuropäische Entwicklung, insbesondere die Verschiebungen durch das dritte Legislativpaket im Energiesektor (Amtsbl. L 211 vom 14.8.2009) sowie die Telekommunikationsrahmenrichtlinie (Amtsbl. L 337 vom 18.12.2009) knapp M. Ruffert Sektorales Wirtschaftsrecht als Teil des europäischen Wirtschaftsrechts, in: ders. (Hrsg.) Europäisches sektorales Wirtschaftsrecht (EnzEur Band 5), 2013, § 1 Rn. 61 ff. Die Rolle des Gesetzgebers wird insbesondere vom EuGH pragmatisch beurteilt, vgl. Urteil vom 6.10.2010, C 389/08 - BASE, Rn. 21 ff., wonach nicht grundsätzlich untersagt sein soll, dass „der nationale Gesetzgeber als nationale Regulierungsbehörde im Sinne der Rahmenrichtlinie“ tätig wird, wenn die spezifischen Anforderungen an diese Regulierungsbehörde erfüllt sind.

${ }_{153}$ Anders gewendet ist die Festlegung des Gesetzgebers auf abstrakte Rechtsregeln, denen dann eine gesetzesanwendende Verwaltung nachfolgt, für die hier besprochenen Konstellationen ebenfalls eine Scheinlösung; dafür aber K. F. Gärditz Die Rolle des parlamentarischen Gesetzgebers im Regulierungsrecht - ein Werkstattbericht, in: Kurth/ Schmoeckel (Hrsg.) Regulierung im Telekommunikationssektor, 2012, 67 (insb. 75 ff.).

${ }^{154}$ Ihre praktische Funktion war in einer Verfassungsordnung ohne Verfassungsgerichtsbarkeit ungleich größer, weil sich der politische Prozess an abstrakten Maßstäben orientieren musste.

${ }^{155}$ Für die europäische Ebene gilt für die flexible Zuordnung von Herrschaftsfunktionen nämliches mit dem Grundsatz des ,institutionellen Gleichgewichts“, vgl. EuGH, Slg. 1990, I-2067 ff. (Rs. C-70-88), Leitsatz 1; Slg. 2008, I-3189 (Rs. C-133/06), Leitsatz 2; auch bei den Handlungsformen ergibt sich eine größere Bandbreite, vgl. H. Wißmann/P. Lange Handlungsformen im europäischen Verwaltungsrecht, in: Leible/Terhechte (Hrsg.) Rechtsschutz und Verfahrensrecht (EnzEur Band 3), i. E., § 31 Rn. 4 ff.; 
laments gegen einen Totalausschluss für bestimmte Entscheidungen, und zugleich ist seine politische Gestaltungshoheit seit jeher vielfältig begrenzt, von der völkerrechtlichen Zustimmungsgesetzgebung ${ }^{156}$ bis zu den europarechtlichen Umsetzungspflichten. ${ }^{157}$ Im Übrigen kann die parlamentarische Gesetzgebungslehre auch von der (hier auch ebenfalls in Betracht kommenden) Volksgesetzgebung lernen, die seit jeher die binäre Entscheidung kennt. ${ }^{158}$ Der entscheidende Maßstab sind so wechselseitige Kontrolle, Formgebung und funktionsadäquate Aufgabenwahrnehmung ${ }^{159}$ - und nach der hier vorgetragenen Überlegung werden diese in der neu gestalteten Abfolge verbessert, nicht verhindert. Damit ist auch klar, dass der Vorschlag nicht einer naiven Vorstellung anhängt, parlamentarische Gesetzgebung wäre per Diskurs schon sachrational; ${ }^{160}$ noch

H. Hill/M. Martini Exekutivische Normsetzung, in: Hoffmann-Riem/Schmidt-Aßmann/Voßkuhle (Hrsg.) Grundlagen des Verwaltungsrechts, Band II, 2. Aufl. 2012, § 34 Rn. $76 \mathrm{ff}$.

156 Art. 59 Abs. 2 S. 1 GG.

${ }^{157}$ Vgl. M. Ruffert in: Callies/Ruffert (Hrsg.) EUV/AEUV, 4. Auflage 2011, Art. 288 Rn. 26 ff.; W. Schroeder in: Streinz (Hrsg.) EUV/AEUV, 2. Auflage 2012, Art. 288 Rn. 76 f.; B. Biervert in: Schwarze (Hrsg.) EU-Kommentar, 3. Auflage 2012, Art. 288 Rn. 23 ff.; EuGH Sammlung der Rechtsprechung des Gerichtshofes 1973, I-161 ff. (Rs. 30/72), Rn. 11.

${ }^{158}$ Vgl. dazu zuletzt M. Möstl/M. Schuler-Harms Elemente direkter Demokratie als Entwicklungsperspektive, VVDStRL 72 (2013), 355 ff., 417 ff. Zur Entwicklung der Bürgerbeteiligung auf kommunaler Ebene F.-L. Knemeyer BayVBl. 2011, 681 ff. S. a. J. Saurer DVB1. 2012, 1082 (1088 f.); zum Einsatz im vorliegenden Zusammenhang bei J. Ziekow Neue Formen der Bürgerbeteiligung? Planung und Zulassung von Projekten in der parlamentarischen Demokratie, Gutachten D zum 69. DJT, 2012, D 111 ff.; J. Rux Direkte Demokratie in Deutschland, 2008, 68 ff.; P. Neumann Sachunmittelbare Demokratie, 2009, $812 \mathrm{ff}$.

159 So die Rationalitätskriterien bei R. Poscher Funktionenordnung des Grundgesetzes, in: Hoffmann-Riem/Schmidt-Aßmann/Voßkuhle (Hrsg.) Grundlagen des Verwaltungsrechts, Band I, 2. Aufl. 2012, § 8 Rn. 23 ff.; skeptisch zur Operationalisierbarkeit M. Cornils Gewaltenteilung, in: Depenheuer/Grabenwarter (Hrsg.) Verfassungstheorie, 2010, $\S 20$ Rn. 36 ff.

${ }_{160} \mathrm{Zu}$ diesem Problemkreis O. Lepsius Die erkenntnistheoretische Notwendigkeit des Parlamentarismus, in: Bertschi u. a. (Hrsg.) Demokratie und Freiheit (AssÖR 39), 1999, 123 (131 ff.); M. Cornils DVB1. 2011, 1053 ff.; zu rechtsstaatlichen und demokratischen Aspekten B. Grzeszick Rationalitätsanforderungen an die parlamentarische Rechtssetzung im demokratischen Rechtsstaat, VVDStRL 71 (2012), 49 (55 ff.). Wenn der Gesetzgeber die Sache voll an sich zieht und auch in der Sache per Dezision entscheiden will, muss er dagegen die Anforderungen, die sonst im Verwaltungsverfahren realisiert werden, im Gesetzgebungsverfahren mit abdecken, vgl. bereits Fn. 142. In dieser Perspektive führt der hier unterbreitete Vorschlag letztlich zu einer Limitierung der Idee vom Einzelfallgesetz als Ausnahmeinstrument. In der einschlägigen Leitentscheidung des BVerfG war die Konstellation insofern anders, als dort zunächst ein reguläres 
viel weniger rekurriert er auf die Idee der ungebundenen Ausnahmegesetzgebung. Ganz im Gegenteil wäre durch die gesetzesgebundene Vorbefassung der Verwaltung konkret gesichert, dass das Einzelfallgesetz nicht zur Abweichung von allgemeinen gesetzlichen Regeln missbraucht werden kann; ${ }^{161}$ der Entlastungseffekt des Verfahrens entstünde (funktional wie rechtsstaatlich angemessen) durch die Verlagerung der Entscheidungsverantwortung auf den Gesetzgeber, was bestimmte Ersatzhandlungen auf Verfahrensebene erübrigte, die im Moment in wenig befriedigender Weise das Feld prägen.

Mit dieser Perspektive liegen die tatsächlichen Probleme des hier formulierten Vorschlags auf den Gebieten von Zuständigkeit und Rechtsschutz. Komplexer ist dabei die Frage der vertikalen Kompetenzverteilung im Mehrebenensystem: Nach der Binnenlogik muss eine funktional begründete Gesetzgebungskompetenz, die auf eine Einzelfallentscheidung

\footnotetext{
Planfeststellungsverfahren begonnen worden war; kritisiert wurde in der abstrakten Normenkontrolle insoweit zu Recht vor allem der „Formenwechsel“ zur Vermeidung von verwaltungsgerichtlichem Rechtsschutz, vgl. BVerfGE 95, 1 (8 f., 12) - Südumfahrung Stendal. Als warnendes Beispiel BT-Drucksache 12/3477, 1 f. (Südumfahrung Stendal): „Der wirtschaftsfördernde Effekt des Vorhabens kann nur erreicht werden, wenn die erforderlichen Maßnahmen so schnell wie möglich verwirklicht werden. Um das zu erreichen, тиß für jeden Streckenabschnitt gesondert festgestellt werden, wie die Planung auf dem schnellsten Weg abgeschlossen werden kann. Insbesondere wegen des Verlassens der Trasse der Stammstrecke Berlin-Lehrte werden auf dem Streckenabschnitt ,Südumfahrung Stendal in besonders starkem Maße öffentliche und private Belange mit der Folge berührt, daß ein entsprechend höherer Zeitbedarf für die Durchführung eines Planfeststellungsverfahrens mit Sicherheit zu erwarten ist. Die hierdurch eintretende Verzögerung von mindestens einem Jahr mit der Folge einer prognostizierten Dauer bis zum Vorliegen des Planfeststellungsbeschlusses für die ,Südumfahrung Stendal' von insgesamt drei Jahren gilt es zu vermeiden. "

${ }^{161}$ Dieses Problem zeigte sich etwa in der Hamburger „lex airbus“ von 2002, vgl. insoweit zu Recht kritisch H. P. Bull NordÖR 2002, 439 ff.; zuletzt J. Lege Verbot des Einzelfallgesetzes, in: Merten/Papier (Hrsg.) Handbuch der Grundrechte, Band 3, 2009, $\S 66$ Rn. 94 ff. Zur Bindung des Einzelfallgesetzgebers an allgemeine Gesetze bereits H. Heller Der Begriff des Gesetzes in der Reichsverfassung, VVDStRL 4 (1927), 98 (121). Eine entsprechende Abwehr des öfter befürchteten gesetzgeberischen Missbrauchs ist der Kern der Systematisierung bei $U$. Hufeld in: Kahl/Waldhoff/Walter (Hrsg.) Bonner Kommentar zum Grundgesetz (Neubearbeitung 2012), Art. 19 Abs. 1, insb. Rn. 20 ff., 78 ff., 154 ff., der zwischen Sondergesetzen und Singulargesetzen unterscheidet und Konsequenz bzw. Folgerichtigkeit als entscheidenden Maßstab für grundsätzlich zulässige Singulargesetze bezeichnet. Im Bereich eines harmonisierten Rechtsrahmens kann sich die Beschränkung des intervenierenden Gesetzgebers schon institutionell ergeben, vgl. einschlägig vor allem EuGH, Urteil vom 3.12.2009, Rs. 424/07 Zugangsrichtlinie, Rn. 65 ff.; eine überschießende Absage an das Gesetz als Handlungsform im Infrastrukturrecht ist damit nicht verbunden.
} 
zielt, der Verwaltungskompetenz nachfolgen. ${ }^{162}$ Der Rechtsschutz fiele bei Bundesgesetzen zunächst unmittelbar in die Zuständigkeit des Bundesverfassungsgerichts. Insofern ist darauf hinzuweisen, dass schon jetzt in etlichen Infrastrukturprojekten das Bundesverwaltungsgericht zuständig ist und also eine Qualitätssicherung qua Instanzenzug nicht stattfindet, so dass hier institutionell betrachtet kein Verlust einträte. ${ }^{163}$ Und bekanntlich gibt es auch kein verfassungsrechtliches Gebot der Vorbefassung der Fachgerichte. ${ }^{164}$ Daher lässt sich ohne weiteres von den Kontrollmöglichkeiten der Gesetzesverfassungsbeschwerde und der abstrakten Normenkontrolle ausgehen, ${ }^{165}$ und ggfs. wäre abzuwarten, ob und wie das Bundesverfassungsgericht wie schon in anderen Fällen Möglichkeiten einer fachgerichtlichen (Vor-) Kontrolle entdeckt, etwa in Bezug auf bestimmte Verfahrensschritte und -rechte. ${ }^{166}$

${ }^{162}$ Es ist darauf hinzuweisen, dass in vielen Fällen Gesetzgebungs- und Verwaltungskompetenz im Bereich der Infrastrukturen ohnehin zusammenfallen, so auch in BVerfGE 95, 1 (22 f.) - Südumfahrung Stendal, weshalb dort der Frage nicht weiter nachgegangen wurde.

$163 \mathrm{Vgl}$. zur Absage an einen zwingende Instanzenzug BVerfGE 4, 74 (LS 6) - Ärztliches Berufsgericht; 11, 232 (233) - Normenkontrollverfahren; 96, 27 (39) - Durchsuchungsanordnung I. Vgl. H. Schulze-Fielitz in: Dreier (Hrsg.) GG-Kommentar, Band 1, 3. Aufl. 2013, Art. 19 Abs. 4 Rn. 94; s. bereits Fn. 102.

${ }_{164}$ Statt aller $A$. Voßkuhle in: v. Mangoldt/Klein/Starck (Hrsg.) Kommentar zum Grundgesetz, 6. Aufl. 2010, Art. 93 Rn. 175.

${ }^{165}$ Zur Erweiterung des verfassungsgerichtlichen Prüfungsumfangs als „Rechtsschutzausgleich" bereits F. Ossenbühl FS Hoppe, 2000, 183 (193); vgl. auch Schneller Legalplanung (Fn. 138), 179; diese Option übersieht Möllers Gewaltengliederung (Fn. 141), 111, bei seiner grundrechtlich begründeten Ablehnung des Einzelfallgesetzes. Schon Forsthoff hat für "Maßnahmegesetze“ eine Vollkontrolle durch das BVerfG gefordert, vgl. GS W. Jellinek, 1955, 221 (235 f.). Die scharfe Absage in BVerfGE 25, 371 - lex Rheinstahl (LS 1: „Der Begriff des Maßnahmegesetzes ist verfassungsrechtlich irrelevant“), v.a. $396 \mathrm{mwN}$, ist insoweit zu spezifizieren: Soweit wie hier vorgeschlagen die Einbeziehung verwaltungsmäßiger Vorentscheidungen ansteht, sind diese nicht von der Einschätzungsprärogative der Legislative gedeckt. Zum Hintergrund J. Lege Verbot des Einzelfallgesetzes, in: Merten/Papier (Hrsg.) Handbuch der Grundrechte, Band 3, 2009, § 66 Rn. 23.

166 Vgl. für die Erweiterung der Feststellungsklage nach entsprechenden Hinweisen in Bezug auf untergesetzliche Normenkontrollen BVerwG NJW 2000, 3584 - Flugrouten; BVerfGE 115, 81 (91 ff.) - Feststellungsklage; siehe auch BVerfGE 70, 35 (57 ff.) Satzungsvertretendes Gesetz. Vgl. H. Goerlich DÖV 1985, 945; M. Möstl in: Posser/ Wolff (Hrsg.) VwGO, 2008, § 43 Rn. 29 ff.; einschlägig zuletzt Th. Groß VerwArchiv 104 (2013), 1 (21 f.). Zur Perspektive des Rechtsschutzes gegenüber den neuen Handlungsformen des europäischen Verwaltungsverbunds allgemein A. v. Arnauld JöR 59 (2011), 497 (514 ff.). 
Zusammengefasst scheint der integrierte Entscheidungsverbund eine Perspektive, im besonderen, aber regulären Fall der Infrastrukturprojekte $\mathrm{zu}$ einem verfassungsrechtlich und funktional adäquaten Vorgehen $\mathrm{zu}$ kommen, dass mit besserem Recht Sachrationalität und Legitimation zusammenführt - gerade weil es den politischen Charakter solcher Entscheidungen erkennt und verfassungsrechtlich rückbindet. Hierüber wird - so hoffe ich - zu streiten sein.

\section{Schluss}

Ich komme zum Schluss: Im Recht der Infrastrukturen geht es um existentielle Voraussetzungen einer freiheitlichen Ordnung - und zugleich ist richtig, dass über Umfang und konkrete Regelung der Infrastrukturen politisch zu entscheiden ist. Die entsprechende Unterscheidung durchzuhalten, ist hier erste Aufgabe des öffentlichen Rechts. Will das Infrastrukturrecht dann konkret nicht mit großflächigen Ausblendungen arbeiten, muss es sich des Dauercharakters seiner Aufgabe annehmen; will es Rationalität und Legitimation in angemessener Weise sichern, sollte es Verwaltungsarbeit und Gesetzgebung neu verbinden. So kann das Recht der öffentlichen Infrastrukturen auf seine Weise zur Zukunftsgestaltung durch Öffentliches Recht beitragen. 
Leitsätze des 2. Referenten über:

\section{Die Anforderungen an ein zukunftsfähiges Infrastrukturrecht}

\section{Einführung}

(1) Das Recht der öffentlichen Infrastrukturen verweist auf grundlegende Herausforderungen des Staats- und Verwaltungsrechts: Funktionierende Infrastrukturen sind Vorbedingung ersten Ranges für eine freiheitliche Bürgergesellschaft im Verfassungsstaat. Die einschlägigen Ordnungsmodelle werden durch fein ausgearbeitete Fachdiskussionen bestimmt, stoßen jedoch zunehmend an Grenzen. Verfassungsrechtliche Vorgaben und verwaltungsrechtliche Systembildung erscheinen als Getriebene statt als Taktgeber des Geschehens.

(2) Der Begriff der Infrastruktur wird im Recht vielfältig, aber letztlich unscharf verwendet. Zutreffend aufgefasst geht es dem Infrastrukturrecht nicht um einen bestimmten, festliegenden Gegenstand, sondern um eine spezifische Fragestellung, nämlich um die Planung (d. h. Konzeption und Entscheidung), Bereitstellung und Pflege von Einrichtungen, die unmittelbar von der Allgemeinheit genutzt werden können oder für weitere Dienste zur Verfügung stehen, die sich an die Allgemeinheit richten. Je nach Eigenart der Infrastruktur ist eine integrierte Betrachtung notwendig, die die Kopplung der Einrichtung mit den Diensten einbezieht.

(3) Insgesamt ist der Sachbereich in Hinblick auf Aufgaben, Akteure und Ordnungsmodelle grundsätzlich offen und politisch gestaltbar. Aus dieser Ausgangslage ergibt sich die Perspektive der Untersuchung: Infrastrukturen und ihre Regelungsmodelle sind in signifikanter Weise mit der Entwicklung des modernen Staates verbunden (II.). Das Infrastrukturrecht lässt sich als dreiphasiges Modell entfalten, wobei die einzelnen Phasen in stärkerer Weise als bisher geschehen in einem Verbundsystem aufeinander bezogen werden müssen (III.). Durch den notwendig politischen Charakter von Infrastrukturentscheidungen ergeben sich abschließend Folgerungen für das Verhältnis von Verwaltungsverfahren und Parlamentsgesetz (IV.) 
II. Entwicklungslinien und Ordnungsmodelle des Infrastrukturrechts

(4) Die Entwicklung von Infrastrukturen und Infrastrukturrecht ist durch eine historisch gewachsene Schichtung verschiedener regulativer Grundannahmen und Leitmodelle gekennzeichnet, die sich nebeneinander bis in die Gegenwart erhalten haben.

(5) In einem langgestreckten Prozess entstehen Staaten in unserem Sinn nicht allein durch Reglement und Gesetz, sondern vor allem auch durch die planmäßig voranschreitende Erschließung des Territoriums. Schon in der frühen Moderne sind dann Versorgung und Kommunikation sowohl ,vor Ort" wie über nationale Grenzen hinweg als typische Kennzeichen von Infrastruktur aufzuweisen. Das Infrastrukturrecht muss dabei seit jeher gegenläufige Zielvorgaben bewältigen, die allgemeinen Bedarf, unternehmerische und technologische Interessen sowie Auswirkungen auf sonstige Rechtsgüter, namentlich die Umwelt, in ein Verhältnis setzen müssen.

(6) Die regulativen Wellenbewegungen haben in den einzelnen Sektoren zu jeweils eigengearteten Infrastrukturteilrechtsordnungen geführt. Die Mischung von Regulierungsansätzen, sowohl zwischen unterschiedlichen Infrastrukturbereichen wie auch innerhalb eines Sektors, ist dabei der typische Befund in Geschichte und Gegenwart. Das Infrastrukturrecht steht so in besonderer Weise für den beweglichen, reaktiven Charakter des Verwaltungsrechts.

\section{Der Verbund von Phasen im Infrastrukturrecht}

\section{Planung: Konzeption und Entscheidung}

(7) Es bestehen typischerweise keine ausdrücklichen verfassungsrechtlichen Vorgaben, ,ob“ öffentliche Infrastrukturen zu schaffen sind. Abgesehen von bestimmten Sonderlagen übernimmt das einfache Gesetz die Aufgabe, solche Pflichten zu kreieren. Von solchen Regelungen sind in gewährleistungsrechtlich regulierten Bereichen auch private Unternehmen betroffen, hier oftmals angeleitet durch europäisches Sekundärrecht. Zu beobachten ist vielfach eine verfahrensorientierte Konkretisierung entsprechender Verpflichtungen.

(8) Abzulehnen ist eine umfassend-allgemeine staatliche Infrastrukturschaffungspflicht. Eine andere Betrachtung verwechselte insbesondere die Legitimationsebenen von Wettbewerbsfreiheit und politischer Gesamtordnung. Es wäre eine Fehldeutung der Konzeption staatlicher Gewährleistungsverantwortung, wenn sie mit einer materiellen Staatsaufgabenlehre verwechselt würde. Ebenso ist daran festzuhalten, dass eine Infrastrukturschaffungspflicht, die durch einfaches Gesetz eingerichtet wurde, keinen unabänder- 
lichen Infrastrukturbestandsschutz auslöst. Nachfolgende Generationen haben weder die Pflicht noch das Recht, vorgefundene Infrastrukturen als gegeben zu nehmen.

(9) Bei der Verwirklichung von Infrastrukturvorhaben sind vorgängige allgemeine Planung und eigentliches Entscheidungsverfahren zu unterscheiden. Das Planungsrecht zielt auf die langfristige, übergreifende Entwicklung von Gesamträumen und Gesamtkontexten; seine Gefährdung besteht darin, in technokratischem Modus scheinbar entpolitisiert vorgebliche Zwangsläufigkeiten zu produzieren.

(10) Für das Entscheidungsverfahren bildet die Planfeststellung einen zentralen Grundtypus. Das Potential der überkommenen Grundidee - förmliche Ordnung mehrseitiger Rechtsverhältnisse durch ein besonderes Verwaltungsverfahren mit dem Abschluss durch eine umfassende Genehmigung konnte in einer demokratischen, grundrechtlich geprägten Rechtsordnung weiter entfaltet werden. Die zwischenzeitlich zu beobachtende Überlastung des Verfahrens mit vielfältigen Erwartungen tritt mit dem Instrument der Plangenehmigung für öffentliche Infrastrukturen besonders klar hervor: Gerade dort, wo die multipolare Lage und die Vielzahl der Abwägungsfaktoren den Aufwand des Verfahrens begründen würden, ist der legitimationsstiftende Teil weitgehend weggekürzt. Dahinter verbergen sich strukturelle Schwächen, die zusammengefasst eine bedenkliche Scheinrationalität und Scheinlegitimation erzeugen.

\section{Bereitstellung}

(11) Bei der physischen Herstellung ist durch das Vergaberecht die Zuteilung öffentlicher Mittel fair zu gestalten. Dabei sind Vertragsgestaltung und-abwicklung bisher rechtlich unterbelichtet geblieben, weil sie zu stark auf punktuelle Vereinbarungen statt auf moderierte Dauerbeziehungen setzen. $\mathrm{Zu}$ zielen wäre hier in intradisziplinärer Offenheit auf neue Mischmodelle, die den multipolaren Charakter solcher Vereinbarungen stärker gewichten und präziser erfassen, namentlich die berechtigten Interessen der infrastrukturabhängigen Allgemeinheit.

(12) Für die Bereitstellung von Infrastrukturen durch private Investoren müssen hinreichend sichere Anreize durch Zuschüsse, Umlagen oder durch Abnahme der entsprechenden Dienstleistungen geschaffen werden. Die entsprechenden Regelungsmodelle, insbesondere das Beihilfenrecht, müssen die Gewährleistung eines angemessenen Zugangs wie auch von Qualitätsstandards berücksichtigen können.

(13) Wenn private Eigentümer von Infrastrukturen gesetzlich verpflichtet werden, diese für andere, insbesondere für Wettbewerber, zu öffnen, entstehen eigentumsrechtlich bedingte Ausgleichspflichten; dies zielt jedoch auf 
Investitionszeiträume, nicht auf „unumkehrbare“ Planungssicherheit. Wenn Eigentümer selbst das Netz nutzen, um Dienste anzubieten, muss der diskriminierungsfreie Wettbewerb mit anspruchsvollen Modellen insbesondere der Preisregulierung durchgesetzt werden; die hier eingesetzte Marktsimulation kann über den normativen Gehalt solcher Regulierungsentscheidungen nicht hinwegtäuschen.

(14) Ein verfassungsrechtlicher oder primärrechtlicher Grundsatz der institutionellen Entflechtung ist abzulehnen: Soweit Markt und Wettbewerb vorgegeben sind, folgt daraus keine Vorgabe, passgenaue Teilnehmer von Teilmärkten ,um“ und ,im“ Wettbewerb zu konfigurieren. Anderes gilt nur als konkrete, sekundärrechtliche bzw. gesetzliche Anordnung des „,Unbundling“.

\section{Pflege der Infrastruktur}

(15) Die Erhaltung und nachlaufende Ertüchtigung bestehender Infrastrukturen muss rechtlich präziser erfasst werden. Die hier zu beobachtende Unschärfe lässt sich in erster Linie als Folgefehler der Ausrichtung auf die Logik privatnütziger Genehmigungen erklären, die für öffentliche Infrastrukturen nicht hinreicht.

(16) Einen Ansatzpunkt für eine angemessene Weiterentwicklung können hier die Modelle bilden, die den Dauercharakter der entsprechenden Verwaltungsaufgabe erkannt haben, etwa in der Zugangs- und Preisregulierung, aber auch im Anlagenzulassungsrecht. Von hier aus ist eine nachlaufende Aktualisierung der entsprechenden Rechtsverhältnisse möglich.

\section{Zusammenfassung: Infrastrukturrecht als Verbundrecht- Infrastrukturverwaltung als Dauerverwaltung}

(17) Das Infrastrukturrecht ist offen für gemischte Arrangements öffentlicher und privater Aufgabenerfüllung. Die Beteiligung an öffentlichen Aufgaben geht jedoch mit erhöhten Pflichtenbindungen einher. Die Sicherung unternehmerischer Freiheit erfolgt hier in erster Linie durch Verfahrens-, Beteiligungs- und Gestaltungsrechte. Insbesondere werden in diesem Feld zukünftig weiter zunehmend komplexe Grundrechtslagen entstehen.

(18) Festzuhalten ist, dass das Infrastrukturrecht als Mehrphasenrecht typischerweise gemischte Regulierungsmodelle etabliert, die in jedem Sektor einem spezifischen Pfad folgen. Es gibt insoweit keinen prinzipiellen infrastrukturrechtlichen Maßstab.

(19) Die öffentliche Hand muss Infrastrukturen im Modus der Dauerverwaltung begleiten. Aufgabe des allgemeinen Verwaltungsrechts ist es hier insbesondere, das begrenzte Wirkungsfeld der rechtsformorientierten Vorge- 
hensweise zu ergänzen: Dort, wo an die Grundentscheidung für eine öffentliche Infrastruktur eine andere Handlungslogik anschließt (also bei der Einbeziehung privater Akteure), muss die weitere Begleitung solcher Projekte als reguläre Aufgabe angenommen werden, die sich typischerweise nicht an den pathologieorientierten Grundmustern des Ordnungsrechts ausrichten kann.

IV. Insbesondere: Das Verhältnis von Verwaltungsarbeit und Gesetz im Infrastrukturrecht

(20) Wenn über öffentliche Infrastrukturen im Regelfall politisch zu entscheiden ist, ist das Öffentliche Recht in vielfacher Weise gefordert: Ihm obliegt, nach der Anschlussfähigkeit sektoraler Teillösungen zu fragen, auf die innere Konsistenz im Sinn einer relativen Pfadabhängigkeit zu bestehen und die verfassungsrechtliche Gesamtsaldierung von Regelungen zu thematisieren, insgesamt also auf Klugheits- wie auf Richtigkeitsentscheidungen zu drängen.

(21) Darüber hinaus sollte das Zusammenspiel von Verwaltungsarbeit und Gesetz verändert und in einen integrierten Entscheidungsverbund überführt werden. Die gesetzliche Entscheidung über konkrete Infrastrukturvorhaben ermöglicht besser abgeschichtete und legitimierte Entscheidungen als das traditionelle Arrangement.

(22) Das Verbot des Einzelfallgesetzes steht einem solchen Ansatz nicht entgegen. Das damit verbundene Schutzkonzept verfehlt die Struktur des parlamentarischen Regierungssystems. Das Grundgesetz macht sich demgegenüber im Grundsatz der Gewaltengliederung in allgemeinerer Weise die Mäßigung der Staatsgewalt und die Verbindung möglichst hoher Sachkunde mit möglichst breiter Legitimation zum Programm - eine zwingende Abfolge von allgemeinem Gesetz und konkreter Verwaltungsentscheidung ist aber nicht das Synonym dieser Vorgabe, sondern nur eine mögliche Ausprägung.

(23) Wenn Verwaltungsarbeit und gesetzgeberische Entscheidung funktional adäquat eingesetzt werden, kann in einem vorgängigen Verwaltungsverfahren die Materie aufbereitet und eine Entscheidung vorbereitet werden. Diese wird dann zur Sache des Gesetzgebers, der über feststehende Alternativen zu befinden hat. Die nachlaufende Verwirklichung des Vorhabens fiele wieder an die Infrastrukturverwaltung zurück, wodurch auch die Verbindung zu den anschließenden Phasen des Infrastrukturrechts sichergestellt wäre.

(24) Dem stehen weder ein ,Verwaltungsvorbehalt" noch eine verabsolutierte Entscheidungsfreiheit des Parlaments entgegen. Beide topoi sind parakonstitutionelle Leitmotive, die in einer ausgestalteten Verfassung durch andersgeartete Begrenzungen ersetzt werden. Der entscheidende Maßstab 
sind wechselseitige Kontrolle, Formgebung und funktionsadäquate Aufgabenwahrnehmung, die sich durch einen neuen Entscheidungsverbund verbessern und mit klaren Entlastungseffekten verbinden lassen. Gelöst werden müssen vor allem die Probleme der Zuständigkeit im Mehrebenensystem und des Rechtsschutzes; für beides lassen sich einleuchtende Regularien finden. 


\section{Aussprache und Schlussworte}

\section{Die Anforderungen an ein zukunftsfähiges Infrastrukturrecht}

Biaggini: Liebe Kolleginnen, liebe Kollegen, es sind erfreulich viele Wortmeldungen eingegangen. Inwieweit spontane Wortmeldungen möglich sind, hängt vom Fortgang der jetzigen Diskussion ab.

Groß: Meine Frage bezieht sich auf beide Referate. Ich denke, dass mittelfristig die Herausforderung weniger der Neubau oder die Planung von Infrastrukturen sein wird, sondern die Frage der Aufrechterhaltung und möglicherweise auch des geordneten Rückbaus jedenfalls gewisser Infrastrukturen. Ich habe den Eindruck, dass unsere Rechtsordnung darauf kaum irgendwelche dem Nachhaltigkeitsgebot entsprechende Instrumente hat, sondern dass das weitgehend entweder ad-hoc oder allenfalls punktuell im Rahmen mittelfristiger Finanzplanungen erfasst wird. Aber gerade wenn die These, dass es sozialstaatliche Mindeststandards gibt, richtig ist, muss man sich überlegen, wie man das konkretisieren kann, insbesondere angesichts voraussichtlich schrumpfender finanzieller Spielräume der öffentlichen Hand, vor allen Dingen auf Landes- und auf kommunaler Ebene. Man beginnt ja erst jetzt, die ganzen Investitionserfordernisse für die Erhaltung der Autobahnen, der Bahnstrecken, der Abwassernetze usw. zu ermitteln. Die Frage ist: Wer könnte in welchem Verfahren halbwegs rational definieren, was Mindeststandards sind? Im öffentlichen Personennahverkehr gibt es zum Beispiel die Regel, dass etwa 300 Leute in einem Ort leben müssen, damit er noch mindestens einmal pro Tag vom Bus angefahren wird. Müsste man solche Regeln nicht verallgemeinern? Kann man das flächendeckend tun? Und auf welcher Ebene müsste das getan werden?

Hellermann: Oliver Dörr hat von der starken Residualverantwortung gesprochen, Herr Wißmann hat eher die Notwendigkeit politischer Entscheidung betont. Zur Klärung würde ich gerne vor allem an Oliver Dörr die Frage richten, wie groß eigentlich der inhaltliche Unterschied zwischen diesen beiden Äußerungen ist.

Soweit über Infrastruktur zu entscheiden ist, ist nach meiner Wahrnehmung in den Referaten zunächst vor allem darüber gesprochen worden, 
inwieweit von politischen und inwieweit von privaten Akteuren zu entscheiden ist, sodann, gegen Ende des Referats von Herrn Wißmann darüber, inwieweit von der Gesetzgebung und inwieweit von der Verwaltung zu entscheiden ist. Die Erörterung der hierzu formulierten These - da bin ich ganz sicher - werden andere noch übernehmen. Sie scheint einerseits von der aktuellen Planung für den Stromnetzausbau inspiriert zu sein und andererseits von Stuttgart 21. Vielleicht mögen auch Verfassungsrichter sich dazu äußern, wie sie sich als künftige Planfeststellungsrichter empfinden. Dazu will ich mich gar nicht weiter äußern. Aber die erwähnte These berührt einen Punkt, auf den ich hinweisen möchte, nämlich den Aspekt der Kompetenzverteilung innerhalb der politischen Ebenen und der politischen Akteure. Hier scheinen mir Fragen von großer Bedeutung zu liegen. Zum einen in der Abgrenzung zwischen Europäischer Union und Nationalstaaten. Was ist wirtschaftliche, was ist nichtwirtschaftliche Tätigkeit im Bereich von Infrastruktur? Wie weit reicht die Privilegierung mitgliedstaatlicher Entscheidungsbefugnisse über Art. 86 Abs. 2 AEUV? Dann im Verhältnis von Bund und Ländern. Das berührt etwa die von Herrn Wißmann angesprochene verfassungsrechtliche Frage nach der Reichweite der Planungsbefugnis des Bundes oder das Verhältnis von Staatsgewalt und Gemeinden (Art. 28 Abs. 2 GG), zum Beispiel bei der Frage der Entscheidung über die Vergabe von Konzessionsverträgen. Die Kompetenzabgrenzungsfrage betrifft dabei sowohl die Frage der Sachentscheidung wie auch vor allem die Frage der Finanzierungszuständigkeit für Infrastrukturmaßnahmen. Vielen Dank.

Burgi: Zunächst hat es mich sehr gefreut, dass beide Referenten nicht der Versuchung erlegen sind, das gegenwärtige Modethema Energieleitungen in den Mittelpunkt zu stellen, sondern auch Infrastrukturen beleuchtet worden sind, die doch ein eher bescheidenes Dasein in der literarischen Behandlung fristen. Während wir etwa alle über Energieleitungsnetze schreiben, verrotten unter unseren Füssen die Abwasserkanäle, die wissenschaftlich bisher nicht Gegenstand näherer Betrachtung gewesen sind. Auch das Straßenrecht ist ein darbendes Rechtsgebiet, dessen sich seit Jahren nur wenige Kolleginnen und Kollegen angenommen haben. Ich sage das auch an die Adresse jüngerer Mitglieder, sich möglicherweise solchen Gebieten verstärkt zuzuwenden. Ergänzen möchte ich noch, dass es beim Bau moderner Infrastruktur natürlich nicht nur um Sozialstaatsund Verbraucherinteressen geht, sondern auch um Wirtschaftsförderung und Wohlstandsgrundlage. Ich hätte dann zwei Bemerkungen, die in erster Linie Herrn Wißmann betreffen. Sie haben sich dafür ausgesprochen, das Infrastrukturrecht als Verbundrecht zu begreifen - über Phasen hinweg und auch jenseits der Träger, Staat einerseits - Private anderer- 
seits. Das ist sehr begrüßenswert. Sie dürfen dabei aber dann nicht stehen bleiben. Sie müssten, wenn Sie sowieso damit anfangen einen Verbund zu entwickeln, von vornherein auch das Infrastrukturfinanzierungsrecht einbeziehen. Denn das größte Problem, das wir in den kommenden Jahren haben werden (jetzt schon sichtbar), ist nicht so sehr, dass wir keine Handlungsformen (Vertrags- und sonstige Instrumente) hätten, sondern dass es an allen Ecken und Enden am Geld mangelt. Sie haben am Anfang gesagt, das sei eine Art Infrastrukturrecht II, das sie zeitlich heute nicht behandeln konnten. Trotzdem würde mich interessieren, ob Sie in dieser Richtung auch schon erste Erkenntnisse haben. Ich will zwei, drei Überlegungen dazu anstellen. Finanzierung durch Nutzer - Stichwort: Gebühren und Beiträge. Das ist ein Rechtsgebiet, das auch eher unterentwickelt erscheint. Es gibt Verwaltungsrichter, die sich damit beschäftigen, aber wissenschaftlich findet dies wenig Interesse, obwohl wir im Gebührenrecht momentan erleben, dass - in Umkehrung des alten Satzes eine Flucht in das Öffentliche Recht stattfindet, weil man mit dessen Hilfe gewissermaßen die Bürger stärker zur Kasse bitten kann, indem man der Kartellrechtskontrolle entgeht. Oder auch das Thema Unterhaltung. Es ist klar, dass die Unterhaltung maroder Straßen, Kanäle usw. letztlich auch über eine Heranziehung von uns allen als Grundstückseigentümer, Mieter usw. über Beiträge erfolgen wird. Auch damit wird man sich politisch und rechtlich befassen müssen. Ganz abgesehen von neuen Finanzierungsformen wie LKW-Maut oder PKW-Maut, über die ich übrigens nicht mit gleicher Eindeutigkeit wie Herr Dörr sofort den europarechtlichen Stab brechen würde. Finanzierung durch Nutzer ist der eine Punkt, der zweite Punkt betrifft die Finanzierung durch neue Trägermodelle. Wir leben in einer Zeit, in der die öffentliche Hand hochverschuldet ist. In der gleichen Zeit ist privates Kapital weltweit auf der Suche nach Anlageorten mit halbwegs attraktiver Verzinsung. Eigentlich wäre dies der ideale Moment, um diese zwei Entwicklungsstränge ein Stück zusammenzuführen. Dazu müsste man Anlagenträgermodelle entwerfen und das wäre etwas für die Bereitstellungsfunktion von Recht. Nun noch zu ihrem Vorschlag zur Handlungsform des Gesetzes. Er ist mir grundsätzlich sympathisch, nicht nur weil ich Gelegenheit hatte, bei der Endlagerstandortsuche daran mitzuwirken, dass die Entscheidung über das abschließende Endlager durch ein Standortplanfeststellungsgesetz getroffen werden soll. Hiergegen brachten die Umweltverbände Art. 19 Abs. 4 GG ins Spiel, mit der Begründung, dass dadurch der Rechtsschutz eben auf die Ebene des Bundesverfassungsgerichts beschränkt werde. Sie haben insoweit weitere Gestaltungsmöglichkeiten angedeutet, mich würde interessieren welche. Mein letzter Punkt betrifft die Übertragbarkeit der Plangesetzgebung auf weniger extraordinäre Fälle, da man nun nicht den Plan für jede Brücke 
durch Gesetz wird feststellen können. Gibt es materielle Kriterien, an denen die diesbezügliche Eignung festgemacht werden kann?

Schoch: Ich möchte mich zunächst bei den Referenten für sachorientierte Vorträge bedanken. Die Referate sind gewissen Modeerscheinungen nicht erlegen, waren informativ und zum Teil - was Herrn Wißmann betrifft auch etwas mutig. Möglicherweise hatten die beiden Referenten die schwierigsten Themen dieser Tagung vom Vorstand erhalten: Es geht um ein ,zukunftsfähiges Infrastrukturrecht“, und wir wissen nicht einmal, ob es ein „Infrastrukturrecht“ überhaupt gibt; dann ist die Frage der „Zukunftsfähigkeit" schon eine gewisse Herausforderung.

Meine erste Bemerkung zur Sache bezieht sich auf die „Dramaturgie“ unserer Tagung. Es wäre, wie sich gezeigt hat, zweckmäßig gewesen, dieses heutige Thema zu behandeln, bevor über Finanzverfassungsrecht und Reformen dazu gesprochen wird. Der ganze Komplex „Aufgaben“ kam gestern bei der bundesstaatlichen Finanzordnung überhaupt nicht vor, stattdessen aber die Idee, die Finanzverfassung als Labor für Experimente zu begreifen. Soll etwa bei finanziellen Engpässen in Kauf genommen werden, beispielsweise zwischendurch die Wasserversorgung einzustellen? Wäre der Komplex „Aufgaben“ zum Ausgangspunkt genommen worden, hätte das „Finanzthema“ eine andere Behandlung erfahren können.

Mit der zweiten Bemerkung bitte ich Herrn Dörr um Aufklärung darüber, warum er, wenn ich ihn richtig verstanden habe, den Entsorgungsbereich ausgeklammert hat. Im Thesenpapier liest man immer wieder ,Versorgung". Doch denken Sie bitte auch an die Abwasserbeseitigung, Abfallentsorgung, Entsorgung abgebrannter Kernbrennstäbe etc.; warum die Engführung auf ,Versorgung“"?

Mit einer kurzen dritten Bemerkung will ich auf die Sicherheit von Infrastrukturen hinweisen. Es geht um die Abwehr von außen kommender Angriffe; das Stichwort ist „Terrorattacken“. Dieser Aspekt kam in den Referaten nicht vor. Gehört diese Thematik nicht auch zu einem Infrastrukturrecht?

Mein Hauptpunkt betrifft, in Übereinstimmung mit Herrn Groß, die Pflege der Infrastrukturen. Über Planung, allgemeine Zielvorgaben und die Errichtung von Infrastrukturanlagen haben wir etwas gehört, kaum jedoch zu deren Pflege. Herr Dörr hat davon gesprochen, dass die Infrastrukturen so etwas wie „Lebensadern“ seien. Zustimmung! Lebensadern müssen jede Sekunde funktionieren, können „Blackouts“ schlecht verkraften. Herr Wißmann hat in dem Zusammenhang von Daueraufgabe, Dauercharakter und Dauerverwaltung gesprochen. Das heißt, wir haben es mit einer permanenten Herausforderung zu tun. Kann das Recht, um an Herrn Burgi anzuknüpfen, vor der Regelung von Finanzierungfragen 
einen Beitrag zur Pflege von Infrastruktureinrichtungen leisten? Worin liegt der Beitrag des Rechts? Wenn ich es recht verstanden habe, haben Sie auf Politik und Klugheit verwiesen. Das heißt doch aber, dass das Recht in diesem Punkt keinen Beitrag zu leisten vermag. Ich will drei Vorschläge unterbreiten und die Referenten fragen, ob dies Ansatzpunkte für eine rechtliche Strukturierung sein könnten. Das Stichwort ist die zunehmende Verrottung von Brücken und Straßen; die Beispiele sind beliebig austauschbar. Vorgestern hat die sog. Bodewig-Kommission erklärt, dass jedes Jahr in Deutschland etwa 7,3 bis 7,5 Mrd. Euro investiert werden müssen, um den Zustand jener Infrastrukturen zu erhalten; ungefähr die Hälfte der Infrastrukturen sei mehr oder weniger ein Sanierungsfall. Was kann das Recht zum Erhalt der Infrastrukturen beitragen? Eine erste Überlegung, die mir während der Vorträge kam: Schaffung von Transparenzpflichten. Wir haben in allen möglichen Bereichen Berichtspflichten, um Transparenz herzustellen und um Diskussionen in Gang zu setzen. Wäre das auch hier ein tragfähiger Gesichtspunkt? Eine zweite Anregung ist die Setzung von Standards; Standardsetzung haben wir in vielen Bereichen, z.B. beim Einsatz der Feuerwehr, bei Notfallplänen, im Umweltbereich, im Lebensmittelbereich bis hin zu Schnellwarnsystemen. Wäre es denkbar, dass das Recht auch zu den Infrastrukturen gewisse Standards setzt und diese mit der Anregung zum ersten Punkt, Transparenz verknüpft? Wenn das Recht, dritte Überlegung, Infrastrukturen nur schwach „zukunftsfähig“ steuern kann, könnte dann nicht ein anderer Weg beschritten werden? Plakativ: Könnte bei der anstehenden Regierungsneubildung nicht ein Infrastrukturministerium geschaffen werden? Es ginge um die Bündelung von Kompetenzen in einer bestimmten Organisation, um die Pflege der Infrastrukturen auf administrativem Wege zu initiieren und um von hier an die Finanzierung ,anzudocken“. Da ich in der Pflege der Infrastrukturen die eigentliche Herausforderung sehe, wäre mir sehr daran gelegen, wenn Sie dazu etwas sagen könnten.

Engel: Die beiden Referenten haben sich an Ihrem Thema gerieben. Herr Dörr war noch etwas vorsichtiger und hat gesagt, dass Infrastrukturrecht eine deskriptive Sammelbezeichnung ist. Herr Wißmann hat noch klarere Worte gesprochen und gesagt: das ist bloß eine Chiffre. Ich frage mich, ob nicht doch mehr dahinter steckt. Ob es also nicht so etwas geben könnte wie ein normatives Proprium, das es rechtfertigen würde zu sagen: das ist tatsächlich ein eigenes Rechtsgebiet.

Wenn das so wäre, dann müssten wir Konflikte finden, die gerade für dieses Rechtsgebiet spezifisch sind. Ich räume Ihnen gern ein, dass viele der Abgrenzungskriterien, die Sie erwogen haben, zu weit und eines vielleicht auch zu eng sind. Zur Angewiesenheit: Auf den Bäcker sind wir auch ange- 
wiesen. Allgemeine Nutzung ist bei den Tankstellen auch richtig. Komplementarität der Güter ist ebenfalls bei den Tankstellen richtig. Ortsgebundenheit ist beim Friseur richtig. Das sind alles Kriterien, bei denen wir sagen würden: Das kann es nicht sein. Natürliches Monopol - daran haben die Volkswirte lang gearbeitet - ist im Zweifel wohl zu eng. Denn das würde heißen, dass es einer billiger kann als mehrere, dass also die Größenvorteile ein extremes Maß erreichen. Das gilt bestenfalls für so etwas wie die letzte Meile in der Telekommunikation. Ich mache es kurz bei dem, was mein halbes berufliches Leben ausmacht - die öffentlichen Güter. Das wäre eine Möglichkeit, aber das ist im Zweifel auch viel zu weit. Da hätten wir die ganze Umwelt oder alle Institutionen auch mit im Spiel.

Aber ich glaube, etwas, das beide von Ihnen erwähnt haben, könnte funktionieren. Nämlich Netze, und zwar Netze im Sinne von Netzexternalitäten. Also je mehr Nutzung für ein Gut ich generiere, umso wertvoller wird das Gut für alle. Das könnte den spezifischen Kern des Infrastrukturrechts ausmachen. Allerdings ist das wohl immer noch zu weit. Denn die Definition der Netzwerkgüter passt auch auf Facebook, Windows und die Sprache. Wir müssen deshalb noch eine weitere, einengende Bedingung finden. Diese zweite Bedingung könnte Ortsgebundenheit sein. Warum Ortsgebundenheit? Warum nicht Sprache, Facebook, Windows? Weil die deutsche Rechtsordnung sich total verheben würde bei dem Versuch, das zu regeln. Und warum geht es bei physischen Netzen, aber nicht nur da? Weil sie ortsgebunden sind, das heißt, weil das Güter sind, bei denen eine Nation sich anmaßen kann zu sagen: Wir machen es in einer bestimmten Weise, und es ist uns unbenommen, dass andere Nationen es vollkommen anders machen. Ein Beispiel aus einem Bereich, den Sie beide im Blick hatten, nämlich dem Verkehr: Wir finden, dass überörtlicher Personentransport über Schienen eine gute Idee ist. Die Amerikaner finden, dass das eine absurde Idee ist und man lieber Flughäfen bauen soll. Das ist eine historisch kontingente Entscheidung jedes Landes, eine Entscheidung, die ein Land deswegen autonom treffen und durch Planung vollziehen kann, weil die Frage, wie die Amerikaner ihren überörtlichen Verkehr organisieren, mit der Frage, wie wir den überörtlichen Verkehr organisieren, nicht furchtbar viel zu tun hat. Ich glaube, hier gäbe es eine Möglichkeit zu sagen, dass wir ein spezifisches Rechtsgebiet haben, und von da ausgehend könnte man dann versuchen, die spezifischen, durch das Recht zu bearbeitenden Konflikte darauf zu untersuchen, wie weit Vergleichbarkeit besteht. Als Folge wären allerdings bestimmte Gegenstände ausgeschlossen, die Sie auch im Blick hatten. Die Schule ist sicherlich kein Netzwerkgut. Der Rückbau eines Atomkraftwerks auch nicht. Der Begriff des Infrastrukturrechts würde das tun, was Begriffe tun sollen: er würde diskriminieren. 
Püttner: Ich möchte ein Wort sagen zum Prinzip der Gleichwertigkeit der Lebensverhältnisse. Herr Dörr, Sie haben es gerade mal so gestreift und eher behauptet, dass es nicht mehr maßgebend sein könne. Ich behaupte das Gegenteil. Ich meine, dass auch heute aufgrund des allgemeinen Gleichheitssatzes die Bürger doch wohl beanspruchen können, überall einigermaßen gleichmäßig mit Infrastruktur ausgestattet zu sein. Das ist, glaube ich, doch sehr wichtig. Im Westen hört man ja manchmal Klagen, dass in den letzten Jahrzehnten die Infrastruktur immer nur im Osten ausgebaut worden sei und im Westen nicht. Egal, ob das nun stimmt, aber es geht doch auf die Vorstellung zurück, dass man grundsätzlich einen gleichen Standard haben möchte. Das wird im Einzelnen - ich denke jetzt an Herrn Burgis Äußerungen - schwierig, wenn wir vor der Tatsache stehen, dass in gewissen Landesteilen die Bevölkerung sich sehr ausdünnt. Frage: Muss dann die Infrastruktur trotzdem auf dem bisherigen Stand gehalten werden oder nicht? Und wenn das in der einen oder anderen Weise geschieht, kann es sein, dass die Bürger in solchen Gegenden, wo - wie zum Beispiel in Schwedt an der Oder - die Bevölkerung um ein Drittel geschrumpft ist, erheblich höhere Abwasser- und Wassergebühren zahlen müssen als in andern Gebieten, weil nämlich die Infrastruktur dort für größeren Bedarf ausgelegt war. Frage: Muss da der Bund ausgleichen und helfen, damit die Bürger in solchen Gebieten nicht benachteiligt werden. Also hier gibt es auch neuere Fragestellungen, die wir, glaube ich, nicht ganz vernachlässigen dürfen. In einem Beitrag war von einem Verbot des Einzelfallgesetzes die Rede. Das hat mich sehr verwundert. Ich weiß, dass es für Grundrechtseinschränkungen in Art. 19 GG so ein Prinzip gibt. Aber in Organisationsbereichen können wir doch nur mit Einzelfallgesetzen arbeiten. Wie wollen Sie denn das Gesetz über die Deutsche Bundesbank so verallgemeinern, dass das kein Einzelfallgesetz mehr ist? Das frage ich Sie also im Ernst.

Meyer, Hans: Ich möchte Herrn Püttner entgegenhalten: es ist vielleicht gar nicht so einfach, eine Autobahn auf eine Spur zurück zu bauen. Ich wollte aber zu Finanzierungsfragen sprechen. Darauf hat Herr Schoch zu Recht hingewiesen. Das hat auch mit der Finanzverfassung zu tun und mit dem, was wir vorher behandelt haben. Ich meine jetzt nicht die Maut, also die Benutzungsfinanzierung, sondern die Finanzierung der Einrichtung selbst und der Unterhaltung durch den Staat. In Deutschland sind alle wichtigen Verkehrswege Bundesangelegenheiten. Das gilt für die Wasserstraßen, die Bundesstraßen wie auch die Bundeseisenbahnen. Nach Art. 104a GG hat der Bund seine Aufgaben zu finanzieren und die Länder ihre Aufgaben. Die Wirklichkeit ist eine andere. Ich nehme an (da bin ich nicht sicher, weil ich nicht nachgeforscht habe), dass beim Rhein- 
Main-Donaukanal - nicht gerade ein vordringlicher Verkehrsweg - die Bayern durchaus mitfinanziert haben. Weil sie damit eine sehr hohe Investitionssumme vom Bund in das Land gelockt haben. Bei Autobahnbauten hört man öfters, dass dieses und jenes Land zugezahlt hat, damit die Autobahn schneller oder gerade in ihrem Land ausgebaut wird. Bei der Eisenbahn haben wir ja den berühmten Fall von Stuttgart 21. Hier hat das Land Baden-Württemberg allein für die Strecke von Wendlingen nach Ulm fast eine Milliarde der Bahn zu zahlen versprochen, obwohl dafür überhaupt kein auch nur fadenscheiniger Gesichtspunkt vorhanden war. Für Stuttgart - Wendlingen 1,5 Millionen - ich bitte Sie, mich jetzt nicht auf eine Million festzulegen, aber das ist so meine Erinnerung. Solche Mitfinanzierung bedeutet natürlich, und das weist auf die Schwäche der Legitimationsfunktion von Parlamentsgesetzen hin, dass diese Legitimation außerordentlich gering ist. Die Eisenbahnstrecken werden nach dem Ausbaugesetz gebaut. Das Ausbaugesetz sieht aber so viele Strecken vor und zudem keine Streckenführungen, sondern nur Anfangs- und Endpunkte einer Strecke, dass die nicht alle in diesem Jahr und auch nicht in den nächsten 5 Jahren gebaut werden können. Das heißt, die Bahn hat freie Entscheidung, welche Strecke sie zuerst baut. Das ist natürlich anders, wenn die Länder ihrerseits Finanzierungen anbieten. Dies ist im Stuttgarter Fall geschehen. Die legitimatorische Wirkung von Parlamentsgesetzen können Sie an diesem Ausbaugesetz auch sehen. Es wird überhaupt keine Streckenführung durch das Parlament beschlossen; es wird nicht beschlossen, wann gebaut wird; es wird nicht darüber debattiert, wie lange gebaut wird und wie teuer es ist; es wird auch nicht darüber debattiert, wie groß eigentlich der Verkehrsbedarf ist, der wird praktisch von der Bahn vorgegeben. Das heißt also, wenn das Bundesparlament darüber behandelt, behandelt es sehr viele wichtige Sachen überhaupt nicht, sondern vertraut dem Bundesverkehrsminister, dass er richtig gerechnet hat.

Möstl: Wir haben heute, was bei dem Thema Infrastruktur auch nicht überraschen kann, von Planung und von Regulierung gehört. Ich möchte diese beiden Punkte Planung und Regulierung nun zusammenführen, indem ich die Frage stelle: Sollten wir Infrastrukturplanung - damit meine ich jetzt Infrastrukturbedarfsplanung, vielleicht auch Fachplanung - in regulierten Sektoren nicht eigentlich als Teil der staatlichen Regulierungsaufgabe begreifen? Ich stelle diese Frage aus vier Gründen. Erstens: Wir haben in der jüngeren Rechtsentwicklung in der Tat Tendenzen in diese Richtung. Das hängt mit dem schon genannten Modethema Energienetzausbau zusammen. Dort hat die Netzagentur mittlerweile ja tatsächlich Fachplanungsaufgaben erhalten und auch Aufgaben im Rahmen der Bedarfsplanung. Jedenfalls zeigt sich eine Tendenz, Bedarfsplanung, Fach- 
planung und Regulierung stärker als Einheit zu sehen und vielleicht entspricht das der Idee von Herrn Wißmann, das Infrastrukturrecht als Verbundrecht zu begreifen. Zweiter Grund: Es scheint mir, dass das Regulierungsrecht, so wie es sich entwickelt hat, in der Tat bezüglich Infrastrukturplanung eine gewisse offene Flanke hat. Regulierungsrecht ist konzipiert für die Öffnung vorhandener Infrastrukturen für den Wettbewerb. Aber es ist etwas hilflos, wenn es um die Frage geht: Wie gewährleistet man eigentlich, dass neue Infrastrukturen entstehen? Im Telekommunikationsbereich ist der Breitbandausbau, der sich ja weitgehend außerhalb des Regulierungsrechts abspielt, ein Beispiel dafür. Auch das Fachplanungsrecht - und das ist der dritte Punkt - gibt keine richtige Antwort, weil es nicht immer ein kohärentes System der Bedarfsfeststellung etabliert. Wichtiger noch, das hat ja auch Herr Wißmann erwähnt: Es bietet vor allem keinen rechten Ort für die politische Entscheidung, ob ein Vorhaben wegen seines Bedarfs wirklich politisch gewollt ist oder nicht. Deshalb haben wir auch die Akzeptanzprobleme mit dem Fachplanungsrecht. Deswegen, vierter Punkt, hat Herr Wißmann vorgeschlagen, den Gesetzgeber stärker miteinzubinden für diese politische Entscheidung. Das möchte ich im Grundsatz unterstützen. Ich meine allerdings, dass der Ort, an dem der Gesetzgeber ins Spiel kommen sollte, vor allem die vorgelagerte Frage der verbindlichen Bedarfsfeststellung ist, d.h. die Entscheidung darüber, ob ein Vorhaben wegen des Bedarfs politisch gewollt ist. Die nachgelagerte Frage, ob das politisch gewollte Vorhaben angesichts entgegenstehender Belange und Rechte auch rechtlich zulässig ist, sollte meiner Meinung nach dann allerdings doch eine Frage sein, deren Prüfung der Verwaltung überlassen bleibt. Sodass ich im Vergleich zu dem Vorschlag von Herrn Wißmann also quasi für eine umgekehrte Reihenfolge plädieren würde, was Gesetzgeber und Verwaltung anbelangt. Danke.

Masing: Mir scheint es sehr anregend und auch überzeugend, den Gesetzgeber in die Planung einzuschalten - in der Tat: zur Stärkung der Legitimation - und dann aber auch das Verwaltungsverfahren mit dem Gesetzgebungsverfahren zu verschränken. Ich habe aber noch nicht ganz verstanden, wieweit damit wirklich eine Legitimationsverschiebung gewollt ist, und möchte hier noch einmal nachfragen: Ein Gesetz enthält ein stärkeres dezisionistisches Element als eine Verwaltungsentscheidung. Ist das gewollt? Und in welchem Umfang soll gegenüber dem bisherigen Verfahren auch das Element der Dezision ermöglicht werden? Ist also auch materiell eine Verschiebung gewollt, oder soll der Gesetzgeber eigentlich nur die Abschlussentscheidung treffen, die bisher die Verwaltung getroffen hat? 
Dasselbe Problem spiegelt sich auf der Ebene des Rechtsschutzes. Es wurde ja schon mehrfach angesprochen: Die Idee, dass die Prüfung eines solchen Beschlusses in erster Instanz und vielleicht alleine beim Bundesverfassungsgericht liegt, lässt auch mir nicht ganz wohl sein. Sie sagten, dass den Gerichten vielleicht etwas einfallen wird, um insoweit zunächst andere Verfahren vorzuschalten. Ich frage mich, ob das dem Gericht einfallen soll oder ob das nicht eine Frage ist, über die schon der Gesetzgeber nachdenken sollte. Dies führt zugleich zurück zu der Frage, inwieweit das dezisionistische Element durch Ihr Konzept gestärkt werden soll. Denn das Verfassungsgericht hat andere Maßstäbe als ein Fachgericht - und soll diese haben; im Ergebnis würde dies also zu einem geringeren Rechtsschutz führen. Vielleicht aber muss man die Verfahrensfragen auch abtrennen. Kann und soll das Verfassungsgericht tatsächlich alle Verfahrensschritte in den Details prüfen - möglicherweise auf der gedanklichen Grundlage, dass diese die inzidenten Rechtmäßigkeitsvoraussetzungen eines Eingriffs in Art. 14 GG (oder in welches Grundrecht auch immer) sind? Dass die Pläne rechtzeitig ausgelegt wurden, dass die Fristen beachtet wurden, dass jeder gehört wurde usw.? Ich glaube, dazu wäre das Gericht seiner ganzen Struktur nach nicht die richtige Instanz. Und wenn sie dafür auf die guten Erfahrungen mit dem Bundesverwaltungsgericht verweisen, muss ich sagen, dass ich dieser Verlagerung des erstinstanzlichen Rechtsschutzes auf ein als Revisionsinstanz konzipiertes Gericht seit jeher kritisch gegenüberstand und nicht glaube, dass hierdurch Rechtsschutz adäquat institutionell eingebunden wird.

Als Schlussperspektive frage ich deshalb, ob es vielleicht nicht angemessener wäre, zwei Gesetzesbeschlüsse zu fordern: Einen ersten Gesetzesbeschluss, der das Verfahren und den Rechtsschutz regelt sowie die Grundstruktur der Planung durch Gesetz vorgibt, und einen zweiten abschließenden Gesetzesbeschluss, der die Entscheidung trifft. Man sieht natürlich, dass auch ein solches Konzept kompliziert und anspruchsvoll ist, und man könnte hier noch weiter überlegen: Sollte auf der ersten Regelungsstufe ein allgemeines Gesetz geschaffen werden, also ein Gesetz, das noch nicht auf ein jeweils konkretes Verfahren zugeschnitten ist? Oder müsste auch das jeweils ein Einzelfallgesetz sein? Das führt zum allerletzten Punkt: Wie weit soll der Anwendungsbereich solcher Entscheidung über Infrastrukturvorhaben durch die einzelfallbezogene Einbeziehung des Gesetzgebers sein und wo endet er? Unendlich weit kann ein solches Modell sicher nicht reichen. All das stellt aber nicht in Frage: Der Grundansatz einer Planung durch das Gesetz hat große Überzeugungskraft!

Spiecker genannt Döhmann: Wir haben zwei ganz ausgezeichnete Referate gehört, die sich mit dem Lebenszyklus von Infrastrukturleistungen befasst 
haben, wohlgemerkt der zu Grunde liegenden Infrastrukturleistungen und nicht der Dienste, die darauf aufsetzen. Darin liegt ein ganz entscheidender Unterschied, der meines Erachtens bei Herrn Engels Überlegungen nicht ganz ausreichend beachtet worden ist.

Ich würde mich gerne mit drei Aspekten ein bisschen näher befassen. Zum einen: Bei Oliver Dörr ist sie als Nachsteuerungsrückholoption angeklungen, bei Hinnerk Wißmann ein Stück weit im Pflegekontext untergekommen - immer wieder die große Frage: Wie sichern wir eigentlich, dass der Staat tatsächlich das, was er tun soll, aber eben nicht vollständig alleine bewerkstelligt, wirklich erledigt - über seine Kontrollmaßnahmen, über seine Begleitmaßnahmen, unter Einsatz welcher Instrumente, die er zur Verfügung hat? Wir haben das unter dem Stichwort des Gewährleistungsrechts in ganz erheblicher Weise schon kennen gelernt. Das verweist darauf, dass wir im Infrastrukturbereich mit Sicherheit die gleichen Nichtwissensprobleme haben und haben werden, die wir in anderen Bereichen der Gewährleistungsverantwortung jetzt schon nicht in den Griff bekommen. Hinnerk Wißmann hat den Versuch gemacht, ob mittels eines stärkeren Zusammenspiels von Gesetzgeber und Verwaltung die Möglichkeit bestehen könnte, zumindest die Startleistung der Infrastruktur diesbezüglich ein bisschen besser zu organisieren. Ich hätte da meine Zweifel. Es müsste eigentlich erfolgversprechender sein, ein ganz anderes Staat-Privater-Modell zu finden. Das Bespiel Straßenbau zeigt, dass und wie man Infrastrukturleistungen in erheblicher Weise bereits jetzt von Privaten erbringen lässt - Herr Burgi hat darauf hingewiesen, dass diese neuen Modelle aber rechtlich bisher überhaupt nicht bearbeitet sind. Dann brauchen wir aber auch eine stärkere staatliche Inklusion im Privaten, um das grundlegende Wissensdefizit in den Griff zu bekommen, um tatsächlich eine kontinuierliche und konsequente Steuerungsleistung vollbringen zu können.

Das bringt mich zu meinem zweiten Punkt. Hinnerk Wißmann hat gesagt, es gäbe keinen Bestandsschutz und kein Bestandsrecht für bestehende Infrastrukturen. Das ist natürlich heikel, wenn ich einen Privaten involviert habe. Denn was mache ich, wenn dieser seinen rechtlichen Pflichten nicht nachkommt? Stichwort: Rückholoption. Enteigne ich? Das preußische Eisenbahnrecht hat seinerzeit eine durchaus kreative Lösung gekannt. Dort war eine Rückholoption von Anfang an vorgesehen: Wenn nicht anständig erfüllt wurde - und zur Beurteilung ließ man bis zu 30 Jahre vergehen -, konnte der Staat von einer Art Verstaatlichungsbefugnis Gebrauch machen, aber ohne anhängende Entschädigungspflichten. Genau das ist auch passiert. Wenn ich das Problem tatsächlich über eine Enteignung regele, ist darin eine Aufforderung an den Privaten enthalten, die Infrastrukturleistung zunächst zu erbringen und sie sich hin- 
terher teuer abkaufen zu lassen. Das ist sicherlich nicht im Sinne des Erfinders.

Kurz mein letzter Punkt. Sehr deutlich angeklungen ist bei beiden Referenten die Abhängigkeit von einer Infrastruktur. Ich glaube aber, ein ganz erheblicher zweiter Punkt ist die Abhängigkeit in einer Infrastruktur, nämlich die Pfadabhängigkeit, die technisch/rechtlich/politisch dadurch entsteht, dass der Staat sich auf ein bestimmtes infrastrukturelles Design einlässt. Meines Erachtens geht die Infrastrukturzukunftsgestaltung so weit, dass der Staat in ganz erheblicher Weise diese Pfadabhängigkeit mit in den Blick nehmen und möglicherweise auch ein alternatives Konzept bereit halten muss, auf das dann weitere andere Dienste aufsetzen können. Vielen Dank.

Knauff: Ich kann an meine Vorredner anknüpfen. Beide Referenten haben die Existenz eines Infrastrukturrechts als Rechtsgebiet abgelehnt. Das ist nachvollziehbar. Insoweit stellen sich die Probleme meiner Meinung nach ähnlich wie auch im Regulierungsrecht, wo wir eine vergleichbare Diskussion - ob es das als eigenes Rechtsgebiet überhaupt gibt - auch schon hatten. Allerdings haben wir auch, das hat Herr Engel vorhin schon gesagt, im Bereich der Infrastrukturen durchaus vergleichbare Fragestellungen, die letztlich auch den Gesetzgeber zwingen, sich jedenfalls zu den gleichen Problemen normativ zu äußern. Genau da sehe ich jetzt allerdings auch ein Problem, denn Infrastrukturen sind, wenn man sie sich im Einzelnen anschaut, immer etwas ganz Konkretes. Das heißt, die gesetzliche Regelung muss einen Rahmen für ganz konkrete Situationen darstellen, die sich auch nicht unbedingt mehrfach wiederholen, wie das in anderen Rechtsgebieten häufig der Fall ist. Da möchte ich ansetzen und anknüpfend an einige Thesen nachfragen: Herr Dörr hat in These 10 etwa auf Art. 14 des AEU-Vertrages Bezug genommen, der eine Funktionsgarantie für die mitgliedstaatliche Daseinsvorsorge und eben auch für die dafür notwendige Infrastruktur enthalte. So sympathisch mir das ist, möchte ich doch noch mal nachfragen: Was ist denn der Maßstab für diese notwendige Infrastruktur? Denn bis jetzt, meine ich, ist man zu Art. 14 AEUV mehr oder weniger der Auffassung, dass es letztlich in weitem Umfang den Mitgliedsstaaten obliegt, festzulegen, was sie für notwendig halten. Dann ist die verstärkende Wirkung natürlich nicht besonders ausgeprägt. Zum anderen, ich knüpfe wieder an Herrn Dörr an, These 15: Die Nachsteuerungs- und Rückholoptionen - das hat Frau Spiecker schon angesprochen - stellen sich natürlich nur dann, wenn Private in die Leistungserbringung einbezogen werden. Allerdings hat dies dann auch gleich besonders schwierige Probleme zur Folge. Als erstes muss sich nämlich der Gesetzgeber die Frage stellen, wie eine entsprechende Regelung 
aussehen soll, damit die Beteiligung für den Privaten hinreichend attraktiv ist und zugleich die Funktionen sicher erfüllt werden können. Herr Wißmann hat dann in These 16 eine nachlaufende Aktualisierung der entsprechenden Rechtsverhältnisse angesprochen. Auch wenn ich jetzt Ihre Thesen vermische, wäre es schön, wenn Sie sich dazu äußern könnten, ob man vielleicht versuchen könnte, einen derartigen Ansatz in eine gesetzliche Regelung - wie auch immer - einzubeziehen. Problem zwei: Die Einbeziehung Privater erfolgt natürlich häufig auch in Vertragsform, sodass wir dann vielfach auf das Zivilrecht verwiesen werden. Auch da wieder der Verweis auf Herrn Wißmann und seine These 11: Sie sprechen dort von moderierten Dauerbeziehungen. Diese lassen sich vielleicht vertraglich durchaus gestalten. Aber dafür müsste man wahrscheinlich eine ausgesprochen anspruchsvolle gesetzliche Grundlage schaffen, denn ansonsten wird der Private sich möglicherweise gegen den Vertragsschluss entscheiden. Dann gibt es keine Dauerbeziehung, die zu moderieren wäre. Schließlich stellt sich natürlich - das kennen wir auch schon aus der Diskussion um den Gewährleistungsstaat generell - die Frage: Wie soll eigentlich die Verwaltung tätig werden? Natürlich haben wir es mit einer Daueraufgabe für die Verwaltung zu tun. Nur, leider - jedenfalls wenn Private in die Leistungserbringung einbezogen sind - verfügt die Verwaltung nicht stets über das Wissen und über alle erforderlichen Informationen. Dann stellt sich die Frage: Wie soll die Verwaltung ihre Aufgaben eigentlich erfüllen und die rechtlichen Vorgaben durchsetzen? Vielen Dank.

Hofmann, Ekkehard: Vielen Dank. Meine Frage bezieht sich auf das Referat von Hinnerk Wißmann, der ja den Mut hatte, tatsächlich auf der Basis einer Defizitanalyse einen Vorschlag zu machen, der durchaus zu Kritik Anlass gibt. Ich möchte in der Defizitanalyse eine kleine Kritik anbringen und beim Lösungsvorschlag eine große. Die Defizitanalyse hat mir zunächst einmal sehr gut gefallen, die Banalisierung von Verwaltungsverfahrensfehlern ist etwas, das viele zu Recht beklagen. Was die Leistungsfähigkeit des Planfeststellungsrechtes angeht, habe ich mir bisher allerdings immer vorgestellt, dass da durchaus noch Potential drin ist, wenn etwa sichergestellt würde, dass eine ordentliche Anhörung durchgeführt wird, in der die Leute Gelegenheit kriegen, ernst genommen zu werden, in der rechtzeitig informiert wird, in der transparent kommuniziert wird, in der am Ende des Verwaltungsverfahrens eine Begründung erfolgt, mit der dann auch alle Betroffenen leben können. Das alles geht bei Hinnerk Wißmann ein bisschen unter oder ein in die Behauptung, das sei scheinrational und scheinlegitim. Da ist mir noch nicht so ganz klar, ob die Scheinrationalität in den Verfahren, in dem Ergebnis der Verfahren 
oder in der Begründung des Ergebnisses liegt. Denn letztlich läuft sein Vorschlag ja darauf hinaus, die aufklärerische rationale Begründung eines Planfeststellungsbeschlusses zu ersetzen durch eine dezisionistische politische Entscheidung. Da wäre ich mir nicht so sicher, dass dieser Wechsel so sympathisch er einem als Demokrat sein kann - eine wirklich überzeugende Lösung wäre. Ich bin mir nämlich nicht sicher, dass da tatsächlich Legitimitätsgewinne zu verzeichnen wären, dass das Finanzierungsproblem (dies ist ja schon angesprochen worden) dadurch gelöst würde oder dass die Scheinrationalität dadurch abgestellt würde. Der Dezisionismus ersetzt dann eben die Rationalität. Das kann eigentlich nicht die Lösung sein. Dann haben mir ein paar Differenzierungen gefehlt. Was eigentlich soll denn nun politisch entschieden werden? Das „Ob“? Welcher Teil des „Wies“? usw. Das ist sicherlich nur der Rücksicht auf die Länge des Vortrags und der vorgerückten Zeit an diesem Nachmittag geschuldet, aber hier würde ich noch gerne ein paar weitere Bemerkungen hören. Vielen Dank.

Dietz: Ich möchte auf Herrn Wißmanns Vorschlag einer gesetzlichen Entscheidung eingehen. Ich denke, das Problem ist nicht die Qualität der Entscheidung. Planfeststellungsbeschlüsse sind sehr ausführlich, sehr ausgewogen und in der Regel auch weitgehend richtig. Das Problem ist doch die Akzeptanz in der Bevölkerung vor Ort, dort wo die Menschen betroffen sind. Ich möchte dazu zwei Aspekte aufzeigen, die jetzt in der Praxis des Senats, dem ich am BayVGH angehöre, auftauchen: Das eine ist die Bestandeskraft der Planfeststellungsbeschlüsse. Aus Sicht des Vorhabensträgers ist die Bestandeskraft ein enormer Gewinn. Denn er kann darauf vertrauen, dass, wenn der Planfeststellungsbeschluss existiert, er auch umgesetzt werden kann. Was wäre hingegen mit einem Gesetz des Landes oder Bundes, wenn beispielsweise nach einer entsprechenden Landtagsoder Bundestagswahl die Mehrheit wechselt und das alte Gesetz plötzlich aufgehoben werden soll? Ich erinnere nur an die Problematik der Laufzeiten der Atomkraftwerke und die Schadensersatzfragen, die sich dort stellen. Ich werde einen etwas anderen Vorschlag mit Blick auf ein Problem der Praxis unterbreiten: Es häufen sich bei uns die Klagen von Kommunen, die vor Ort Belegenheitsgemeinden sind, wo also die Stromtrassen durchlaufen und die vorher im Einwendungsverfahren entweder auf Einwendungen verzichtet oder ausdrücklich sogar die Zustimmung zum Verfahren erklärt haben. Dann gehen einige Jahre ins Land. Sorgfältig prüft die Behörde im stillen Kämmerchen, was alles an Einwendungen kam. Dann ergeht der Planfeststellungsbeschluss und alle sind überrascht: „Ach, da war ja was!“ Wenn dann die Mehrheit im Gemeinderat gewechselt hat, kommt die Idee, doch Klage zu erheben. Diese Klagen werden 
tatsächlich erhoben, einfach als Ventil für den Unmut der Bevölkerung vor Ort. Ich möchte daher auf die Vorredner eingehen, die meinen, wir könnten das Planfeststellungsverfahren ertüchtigen. Wie wäre es denn damit, dass zum Beispiel Zwischenergebnisse verpflichtend veröffentlicht werden? Dass zum Beispiel Einwendungen institutioneller Einwender also nur von öffentlichen Rechtsträgern oder zum Beispiel von Umweltverbänden - publiziert werden, zum Beispiel in den örtlichen Amtsblättern? Damit die Bevölkerung auf dem Laufenden ist, sieht, was derzeit diskutiert ist, und dann quasi kontinuierlich der Faden des Planfeststellungsverfahrens bis zum Planfeststellungsbeschluss fortgeführt wird. Auf diese Weise könnte man vielleicht die Akzeptanz erhöhen und gleichzeitig das Bundesverfassungsgericht vor weiterer Belastung schützen. Vielen Dank.

Wallrabenstein: Vielen Dank für die beiden sehr anregenden Referate. Gerade Hinnerk Wißmanns Fragestellung nach einem zukunftsfähigen Infrastrukturrecht habe ich so verstanden, dass sie danach sucht, was man aus den im Einzelnen beleuchteten Feldern für andere Bereiche des Verwaltungsrechts oder für das allgemeine Verwaltungsrecht ableiten kann. In dem Sinne ist meine Frage gedacht: Was lässt sich beispielsweise im Sektor „Planung“ für andere Regelungsbereiche ableiten, wenn man sich einen bestimmten Bereich anschaut: Nimmt man etwa die Versorgungsplanung im Gesundheitswesen oder die Pflegeplanung, dann besteht dort eine recht intensive Debatte darüber, wie der Bedarf, der beplant werden soll, festgestellt wird. Diese Bedarfsfeststellung erfolgt nicht durch den Gesetzgeber. Vielmehr ist vorgesehen, dass der Bedarf von Fachinstituten, von Interessenverbänden, von Körperschaften festgestellt wird. Wer dabei welche Kompetenzen haben soll, ist letztlich die große Frage. Und natürlich die Frage, wie gut die Bedarfsfeststellung funktioniert. Lässt sich aus so einer Debatte etwas ableiten für andere Bereiche? Hat diese Herangehensweise Erkenntniswert? Weil Hinnerk Wißmann das auch angesprochen hat und ich gestern schon die Gelegenheit hatte, zum Schulbereich Überlegungen anzustellen: Welchen Erkenntniswert hat es für das weitere Verwaltungsrecht, wenn wir sagen, dass es so etwas wie eine Schulnetzplanung geben soll, aber der Bau von Schulen in kommunaler Hand und der Betrieb (die Lehrerstellen) in Landeshand liegt, so dass letztlich das Land entscheiden kann, welche Schulen es gibt, weil es entscheidet, welche Lehrerstellen bewilligt werden, was eine bestimmte Zahl an Schülern voraussetzt (wir hatten gestern das Beispiel). Was kann man aus dieser Interessen- und Zuständigkeitslage ableiten für andere Bereiche? Kann sie ein Beispiel sein, wie Planung gut oder eben schlecht funktioniert? Diese Art von Fragen kann man etwa für den Bereich Pflege 
der Infrastruktureinrichtungen, der im Referat und in der Diskussion bereits mehrfach angesprochen wurde, erwägen: Was ist der Wert, wenn man die konkreten Dauerpflegeregelungen, die es gibt, so gut oder schlecht wie sie sind, anschaut? Kann man daraus etwas für andere Bereiche ableiten? Da scheint mir auch der angesprochene Vergleich zwischen privatbetriebenen und öffentlich betriebenen Infrastruktureinrichtungen interessant $\mathrm{zu}$ sein. Beispielsweise kann man im Atomrecht schauen, wie dort die Nachführungs- und Instandhaltungsregime der privaten Atomkraftwerkbetreiber geregelt sind. Wie funktionieren sie? Was ist daran gut oder schlecht? Kann man daraus etwas ableiten für öffentlich geführte Unternehmen? Sollten sie auch Betreiberpflichten haben oder nicht? Reicht da die Baulast oder muss es präziser sein? Das wären einige mögliche Fragen. Ich würde mich freuen, wenn jemand aus dem einen oder anderen Bereich solche Ableitungen treffen könnte.

Fehling: Ich möchte noch einmal bei der Frage anknüpfen, welche rechtlichen Instrumente wir entwickeln können, um einem schleichenden Verfall vorhandener Infrastruktur entgegenzuwirken oder auch neue Infrastruktur (bei Pflege, bei Kitas) bedarfsgerecht auszubauen. Mir leuchtet es sehr ein, dass es, wie Hinnerk Wißmann betont hat, einen direkten Anspruch des Einzelnen auf Schaffung von Infrastruktur wohl kaum geben kann. Doch habe ich den Eindruck, dass individuelle Rechte doch eine gewisse Anstoßfunktion entfalten könnten. Art. 36 der Grundrechtecharta scheint, jedenfalls von der Überschrift her, den Zugang zu Diensten von allgemeinem Interesse grundrechtlich zu garantieren. Wenn man die Norm dann weiter liest, klingt es allerdings nicht nach einem Grundrecht auf Schaffung von Infrastruktur. Aber immerhin wird man aus der Norm, wenn man sie wie geboten ernst nimmt, eine Stärkung des Aspekts individuellen Teilhabe herauslesen müssen. Ließe sich dieser europarechtliche Teilhabeansatz vielleicht verknüpfen mit der auf nationaler Ebene bekannten numerus-clausus-Rechtsprechung (natürlich auch mit all den Begrenzungen, die diese Rechtsprechung enthält), um dann - ein Konzept, das wir aus anderen Bereichen des Unionsrechts kennen - den Einzelnen ein Stück weit zu instrumentalisieren für die Durchsetzung der zunächst objektiv-rechtlichen Infrastrukturgewährleistungsverantwortung? Bei manchen Infrastrukturen erschiene mir das gar nicht so schwierig - Stichwort Pflegeplätze oder (je nach Generation: noch näherliegend) Kita-Plätze. Bei anderen Infrastrukturen wird es sicherlich problematischer. Aber denken wir an ein Betreibermodell, wie beispielsweise bei den Berliner Wasserbetrieben. Dort werden die Leistungen immer schlechter, die Infrastruktur verfällt. Gäbe es insoweit für den Einzelnen Klage- oder sonstige Interventionsmöglichkeiten, zwar nicht um direkt konkrete Maß- 
nahmen zu erzwingen, aber doch dafür zu sorgen, dass der Staat die Probleme nicht einfach ignorieren kann? Vielen Dank.

Kugelmann: Vielen Dank. Oliver Dörr hat den Begriff Infrastrukturrecht deskriptiv verstanden. Mit guten Gründen hat er die Infrastrukturverantwortung dann in Zusammenhang mit der Daseinsvorsorge gebracht. Da wollte ich unionsrechtlich nachfragen, weil diese Dienstleistungen von allgemeinem wirtschaftlichem Interesse meines Erachtens vielleicht doch nicht „so" geklärt und „so" weit gehend abgesichert sind, wie das angedeutet wurde. Ich würde mir das wünschen, sehe aber durchaus, dass im Spannungsfeld der Daseinsvorsorge zur Binnenmarktlogik gerade auch im Richtlinienbereich - und vieles oder vielleicht beinahe alles, wovon wir reden, ist auch richtliniengeprägt - die Richtlinien doch erhebliche Auswirkungen haben. Dies betrifft, nicht nur die Dienste, sondern rückwirkend auch die Infrastrukturen, die den Diensten zu Grunde liegen. Deshalb stellt sich die Frage, ob die Daseinsvorsorge wirklich gesichert ist auch in den Bereichen, die stark richtliniengeprägt sind oder ob nicht der Zugang zu den einschlägigen Dienstleistungen verstärkt von der liberalen Logik des Binnenmarkts geprägt ist. Die zweite Anmerkung betrifft Herrn Wißmann. Sie haben das Gesetz als Vorschlag ins Spiel gebracht. Das wird sicherlich nicht alle Fälle betreffen können. Auch wenn man dem Vorschlag folgen möchte, müssen Eingrenzungen angebracht und der Anwendungsbereich sicherlich begrenzt werden. Die Frage ist, ob es - das ist verschiedentlich schon angeklungen - ein aliud durch bessere Partizipation gibt. Nehmen wir einen Bereich, den Sie aus Zeitgründen nicht auch noch behandeln konnten: Informationsmanagement. Wenn man sich ein Infrastrukturrecht als Verbundrecht vorstellen könnte, zumindest in gewissen Bereichen, dann würde ich dem Informationsmanagement gerade unter dem Gesichtspunkt der Akzeptanz, der bereits angesprochen wurde, erhebliche Bedeutung beimessen. Ist dann vielleicht die Verbesserung der Partizipation auch ein aliud? Um es anders zu sagen: In diesem Bereich, in dem es funktionieren kann - zumindest besser funktionieren kann als es bisher funktioniert hat - brauche ich dann den Gesetzgeber gar nicht in die Pflicht zu nehmen? Vielen Dank.

Faßbender, Kurt: Ich habe eine Frage zum Referat von Herrn Dörr und da namentlich zur These 17. Herr Dörr, Sie haben ja dankenswerterweise das Unionsrecht analysiert, was es für unser Thema Infrastrukturrecht hergibt, und sind dann in Ihrer These zum Ergebnis gekommen, dass das Unionsrecht hier materiell-rechtlich eine nur begrenzte Steuerungskraft habe. Vor diesem Hintergrund haben Sie gar von einem ,infrastrukturpolitischen Autonomieanspruch der Mitgliedsstaaten“ gesprochen. Die 
Frage ist, ob das nicht das Unionsrecht allzu sehr in seiner Bedeutung reduziert. Sie haben selbst das Binnenmarktrecht der Union als Einflussfaktor angesprochen. Hier möchte ich besonders den Blick auf die Energiebinnenmarktrichtlinien lenken, wo seit geraumer Zeit im energierechtlichen Schrifttum diskutiert wird, welche Konsequenzen sich daraus mit Blick auf die Netzausbaupflichten ergeben. Dann haben Sie selbst die Kompetenz für die transeuropäischen Netze angesprochen. Da haben wir jetzt seit einigen Wochen eine Verordnung zu Leitlinien für die transeuropäische Energieinfrastruktur. Daraus ergeben sich doch recht weitreichende Vorgaben für unser Planungsrecht in diesem Bereich, zumindest für die dort geregelten Vorhaben von gemeinsamem Interesse. Das geht sogar so weit, dass die Übertragungsnetzbetreiber, wenn sie in dem vorgegebenen Zeitrahmen die Netze nicht gebaut bekommen, akzeptieren müssen, dass dies ausgeschrieben werden kann. Dies sind schon weitreichende Regelungspunkte.

Da möchte ich überleiten zu Ihrer These 23. Die sagt uns mit Blick auf die Zukunft, dass die Anforderungen an ein zukunftsfähiges Infrastrukturrecht durch internationale Lösungen sicherzustellen seien. Wäre da nicht das Unionsrecht der naheliegende Anknüpfungspunkt, gerade mit dieser Kompetenz für die transeuropäischen Netze, wenn es um grenzüberschreitende Infrastrukturnetze geht?

Ein Punkt hat mich schließlich besonders geschmerzt: dass das Umweltrecht so gar nicht zur Sprache kam. Nicht weil es mich besonders interessiert, sondern weil es einen ganz erheblichen Einfluss auf unser Thema hat. Sie haben selbst die Waldschlösschenbrücke in Dresden angesprochen, und zwar wegen des UNESCO-Weltkulturerbes. Da sind die Dresdner ja noch mit zurechtgekommen. Aber woran sie noch heute tragen müssen, ist an der Kleinen Hufeisennase, die dazu führt, dass da nur 30 gefahren werden darf. Das geht ja noch. Aber die dahinter stehenden Vorgaben des europäischen Naturschutzrechts können mitunter Vorhaben der Infrastruktur auch verhindern. Herr Burgi hat die Abwasserbeseitigung angesprochen, da auch Sie den Punkt mit angesprochen haben: Hier haben wir eine Richtlinie über kommunales Abwasser aus dem Jahre 1991, die sehr konkrete Vorgaben enthält, etwa zu konkreten Anschlusspflichten usw. usf. Auch vor dem Hintergrund wollte ich kritisch nachfragen, ob man die These von der geringen Bedeutung des Unionsrechts vielleicht etwas relativieren sollte.

Weiß, Norman: Ich möchte mich dem Dank für zwei sehr interessante Referate anschließen und meine Frage an Oliver Dörr richten. Beide Referenten haben - wie ich finde richtigerweise - von der Raumorientierung gesprochen, um die es hier geht. Oliver Dörr hat dann auch den Schritt 
ins Völkerrecht gewagt und dort zunächst auch raumorientierte Regime angesprochen - Flussregime beispielsweise - und dann mit These 21 den mutigen Ausblick ins Internet gewagt. Meine Nachfrage bezieht sich genau hierauf. Wäre es sinnvoll, das Internet als weiteren herrschaftsfreien Raum zu betrachten, der dem Zugriff von Territorialstaaten entzogen ist und bleiben soll? Das würde zum Teil an das anknüpfen, was Herr Engel gesagt hat. Kann man das als global common definieren, das dem Zugang und der Nutzung aller offen steht und gäbe es da - schwierige Frage, ich weiß - taugliche Regelungsansätze. Und wenn man erfolgreich wäre, das als Anschlussfrage, wäre das ein weiterer Schritt hin zu dem, was gelegentlich unter dem Thema „Weltinnenrecht" thematisiert wird, aber durchaus auch verwaltungsrechtliche Züge hätte und damit wieder gut zu dem anderen passen würde. Vielen Dank.

Nowrot: Herr Dörr hat um Nachsicht dafür gebeten, dass er im Rahmen seines Referates auch auf die völkerrechtlichen Aspekte der Thematik eingegangen ist. Deswegen bitte auch ich zunächst einmal um Nachsicht, dass ich - ebenso wie bereits mein Kollege Weiß - hierzu ebenfalls noch eine Frage habe. Herr Dörr, Ihre diesbezüglichen Ausführungen haben Sie mit der Aussage begonnen, dass die rechtliche Determinierung eines zukunftsfähigen Infrastrukturrechts durch das Völkerrecht doch eher gering sei. Ihre Darstellung hat sich auch im Wesentlichen - wenn ich das richtig verstanden habe - auf die rechtlichen Rahmenbedingungen der Durchführung und Nutzung von Infrastrukturprojekten bezogen. Ich frage mich aber doch, ob sich damit die normative Steuerungswirkung des Völkerrechts in diesem Bereich erschöpft.

Es ist ja anerkanntermaßen so - und Sie haben dies auch ausgeführt -, dass im innerstaatlichen Bereich der Verfassungsauftrag für die Gewährleistung einer funktionsfähigen Infrastruktur und damit auch die Verortung der Letztverantwortung des Staates für die Infrastruktur einerseits auf das Sozialstaatsprinzip sowie andererseits auf die Grundrechte zu stützen ist. Könnte man diese Überlegungen aber dann nicht vielleicht auch auf die völkerrechtliche Ebene und ihre Regelungsstrukturen übertragen und von so etwas wie einer Infrastrukturverantwortung durch Völkerrecht bzw. auf der Grundlage des Völkerrechts sprechen? Ich möchte hier gar nicht auf die gelegentlich bereits diskutierte Frage eingehen, ob das Völkerrecht schon in eine Entwicklungsphase eingetreten ist, in der sich auch auf internationaler Ebene so etwas wie sozialstaatliche Ordnungsideen positivrechtlich nachweisen lassen. In jedem Fall kämen hier aber - im Sinne der zweiten „Säule“ im innerstaatlichen Bereich - die Menschenrechte in Betracht. Auch im Völkerrecht ist ja allgemein anerkannt, dass die Menschenrechte nicht nur abwehrrechtlichen Charakter 
haben, sondern sich aus ihnen auch staatliche Schutzpflichten ableiten lassen. Wenn man dies in Betracht zieht, wäre die entsprechende Regelungsdichte im Völkerrecht doch nicht ganz so gering, sondern würde sich auch auf die Grundlagen eines entsprechenden Sicherungsauftrags des Staates beziehen.

Ganz kurz am Schluss noch zur praktischen Relevanz dieser Überlegung. Zwar müssen rechtswissenschaftliche Überlegungen nicht notwendigerweise auch zu einem praktisch relevanten Ergebnis führen. Aber wenn es ein solches gibt, muss man damit ja auch nicht hinter dem Berg halten. Namentlich im Bereich des internationalen Investitionsrechts, aber auch des Welthandelsrechts, gibt es entsprechende Rechtsvorgaben, die wenngleich nicht unmittelbar mit dem Unionsrecht vergleichbar - ebenfalls auf entsprechende Liberalisierungsmaßnahmen abzielen, die zur Konsequenz haben können, dass man sich auch auf derselben Rechtsebene fragen muss, in welcher Weise - gleichsam im Sinne eines normativen "Gegengewichts“ - auch die staatliche Letztverantwortung für die Infrastruktursicherung positivrechtlich im Völkerrecht abgestützt werden kann. Hier kämen in erster Linie die Menschenrechte in Betracht, sodass man vor diesem Hintergrund zumindest ansatzweise von so etwas wie einer auch völkerrechtlichen Gewährleistungsverantwortung sprechen könnte. Danke schön.

Zacher: Vielen Dank. Ich würde gerne noch ein paar Bemerkungen zur Tagung als Ganzes machen. Ich bin in meinem Urteil sehr gespalten. Ich habe jedes Referat eigentlich genossen: als mehr oder weniger - meistens mehr - perfekte Leistung, als gutes Handwerk und voller guten Ideen. Trotzdem frage ich mich nach der Architektur der Tagung. Kann man so über das Thema „Zukunftsgestaltung durch öffentliches Recht“ reden? Denn was wir sagen könnten und was Sie sagen konnten war ja, dass man durch Recht gute Wege bahnen kann, dass einzelne der erörterten Materien eine gute Zukunft haben und die Anliegen, die von ihnen betroffen sind, auf eine gute Bahn gesetzt wurden. Das kann man. Aber was dann noch bleibt ist die Politik. Alle rechtlichen Regelungen sind eingebettet in Wirklichkeiten - schon ehe sie Recht werden und nachdem sie Recht geworden sind. Und eine ganz besondere dieser Wirklichkeiten ist die Politik: Davon war überhaupt nicht die Rede, obwohl auch die Politik in öffentlich-rechtlichen Ordnungen lebt. Ich glaube, dass wir, wenn wir alles durchgehen, relativ wenig wissen, von welcher Zukunft wir in diesen zwei Tagen geredet haben. Dies ist so, weil wir nicht von der Politik selbst geredet haben. Ich muss da auf mein zentrales Lebensfach zurückkommen, auf das Sozialrecht. Da beobachte ich das umso schmerzlicher immer mehr. Von Normen werden Lösungen erwartet. In Wirklichkeit lassen 
Normen aber unendlich Raum - sei es in der Normgestaltung, sei es in dem Drumherum der Normverwirklichung. Die Wirklichkeit wuchert immer wieder um die Normen. Sie ist sehr ungehorsam gegenüber den Normen. Damit umgehen muss die Politik. Aber wir wissen viel zu wenig, wie wir zu einer guten Politik kommen. Politik bedeutet ja in diesem Fall nicht nur Politiker, Behörden, Parlamente usw., sondern Politik umfasst natürlich auch den Wähler und die öffentliche Meinung, über dieses ganze Zusammenspiel mitsamt dem Umgang mit der Zukunft. Wenn wir uns jetzt - und damit schließe ich - vor Augen führen, dass Politik immer in der Gegenwart liegt, weil in der Gegenwart gewählt wird, in der Gegenwart geherrscht wird, in der Gegenwart sich aufgebäumt und aufgespielt wird. Alles lebt in der Gegenwart. Eigentlich kümmert man sich um die Gegenwart und nur ganz nebenbei um die Zukunft. Kann das Recht da etwas tun, um der Zukunft mehr Sorge, mehr Geltung sowie mehr Rücksicht zu verschaffen? Es wäre schön gewesen, wenn wir darüber jetzt auch etwas mehr wüssten.

Biaggini: Vielen Dank, Herr Zacher, für diese sehr bedenkenswerten Worte. Die Politik ist in der Tat eine wichtige Dimension, aber auch eine Dimension, die nicht ganz so leicht zu fassen ist. Darüber könnte man natürlich jetzt noch lange diskutieren. Aus Zeitgründen muss ich das Wort jetzt allerdings weitergeben an die beiden Referenten. Es spricht, wie üblich, zuerst Herr Wißmann als unser zweiter Referent.

Wißmann: Ich beginne mit einem doppelten Dank, der sich zunächst an den Vorstand richtet, dass er mir und uns dieses Thema übertragen und damit Gelegenheit zu vertiefter wissenschaftlicher Arbeit inmitten der Alltagsstürme gegeben hat. Der zweite Dank geht an Sie für Kritik und Zuspruch in der Diskussion. Von beidem lebt man ja, als Wissenschaftler wie als Mensch.

Ich möchte versuchen, die Debatte für mich etwas zu sortieren: Ich beginne mit dem Begriff der Infrastruktur und der Rückfrage von Herrn Engel, ob wir nicht doch ein materielles Infrastrukturrecht eingrenzen könnten, wenn wir nur lange genug suchten - nämlich das Recht der Netzexternalitäten, wenn wir es mit der Ortsgebundenheit koppeln. Ich folge dem nicht. Meine Rückfrage wäre, ob nicht mit einer solchen Perspektive vorschnell ein materielles Ordnungsmodell verbunden wird, nämlich das eines bestimmten Wettbewerbskonzepts. Nun ist das aber gerade meine Pointe: Ich glaube, dass es ein vorgeordnetes Grundmodell im Bereich der Infrastruktur nicht gibt. Eine solche Vorstellung verwechselt gerade die Ebenen von verfassungsrechtlicher Gesamtordnung und Wettbewerb. Nur am Rande: In den USA wird eben nicht nur geflogen, sondern die armen 
Leute fahren Bus. Wäre das bei Ihnen schon wieder draußen, weil es nicht ortsgebunden ist? Oder würden die Busstationen ausreichen? Ich glaube, da kämen wir vom hundertsten ins tausendste.

Mein zweiter Punkt betrifft die Frage des Themenzuschnitts, also der Konzeption von Phasen und der einzelnen Fragestellungen des Infrastrukturrechts. Das sind zunächst die Beiträge von Herrn Burgi, Herrn Meyer und Frau Spiecker. Ich gebe Ihnen völlig recht: Ich hätte auch eine Dreiviertelstunde ausschließlich über Finanzierung sprechen können. Man kann dieses Thema alleine von dort aus aufbauen, und das sind elementare, gerade auch politische Fragen. Ich würde aber hoffen, dass man diese Fragen mit meinem Konzept eines phasenorientiertes Rechts verbinden kann, das auch diesen Bereich dann ergänzend aufarbeiten muss.

Es hat mich gefreut, dass es dann doch überwiegend auf Zustimmung zu stoßen scheint, dass es sich hier um eine Daueraufgabe handelt und dass wir im Schwerpunkt gar nicht so sehr von neuen Infrastrukturen, sondern von den Infrastrukturen, die wir bereits vorhalten, sprechen müssen. Die Pflege ist ein materiell wichtiger Punkt. Herr Schoch hat das nochmals besonders ins Spiel gebracht, aber auch die Beiträge von Herrn Möstl, Herrn Knauff und Frau Wallrabenstein sind in unterschiedlicher Weise damit umgegangen. Ich kann hier nur in aller Kürze sagen: Natürlich sind Sicherheitsaspekte dabei elementar wichtig. Bloß erschöpft sich der Aspekt der „Pflege“ nicht darin. Das ist, glaube ich, der Fehler der alten Ordnungsmodelle, dass sie auch bei öffentlichen Infrastrukturen von einer privatautonomen Nutzung von Genehmigungen ausgehen. Hier würde ich zu einer grundlegenden Veränderung der Perspektiven raten. Standardsetzung in Bezug auf Transparenz wie auch auf Qualität ist hier ein wichtiges Thema - so etwa in der interdisziplinären Zusammenarbeit im Bereich von DIN-Standards. Es wird einem ganz anders, wenn man mit Ingenieuren spricht: Was die meinen, wie verbindlich solche Standards sind und warum. Da hat das deutsche Recht eine nach wie vor große Aufgabe in Aufklärung und Dialog.

Der dritte Punkt ist die Frage: Planfeststellungsverfahren oder Einzelfallgesetz? Zunächst einmal war mein Eindruck, dass viele Staatsrechtler schon ins Wochenende gegangen sind, weil die Kritik eher verhalten war und im verwaltungsrechtlichen Modus erklang. Trotzdem haben Herr Hofmann, Herr Dietz und Herr Kugelmann doch eher die Ertüchtigung des bisherigen Planfeststellungsverfahrens angemahnt. Ich halte das alles für richtig, dass man das schöner machen kann. Bloß glaube ich, der Pfad, auf dem Sie sich bewegen - noch extensiver aufzuarbeiten, noch mehr zu beteiligen - löst das Problem nicht, das dort im Grunde besteht. Ich verweise auf eine Diskussionsbemerkung von Herrn Waechter vor einigen Jahren, der damals sagte, dass bei diesem ganzen Verfahren doch 
in Wahrheit die Entscheidung immer schon gefallen sei und es nur noch darum ginge, ob noch eine Lärmschutzwand oder ein Kaninchenschutzbunker gebaut wird. Alles andere sei Fassade. Und Herr Waechter ist immerhin auch in einem OVG-Senat tätig. Insofern war mir das eine Ermutigung, mich auf das Einzelfallgesetz als Alternative zu konzentrieren. Einige Beiträge habe ich eher als Bestätigung verstanden - Herrn Meyer, Herrn Möstl, auch Frau Spiecker insoweit. Allerdings, Herr Püttner, geht es da schon um Grundrechtseingriffe. Wenn eine Straße, ein Flughafen oder ein Atomendlager konkret durch ein Gesetz beschlossen wird, dann ist das nicht nur Staatsorganisation, sondern betrifft Anlieger und Nachbarn. Insofern kommt man, glaube ich, so schnell aus der Nummer nicht heraus. Ich halte aber trotzdem Art. 19 Abs. 1 Satz 1 GG nicht für eine sinnvolle Perspektive, damit umzugehen. Herr Burgi hat auf das Problem des Art. 19 Abs. 4 GG in diesem Zusammenhang hingewiesen. Ich glaube, man kann hier die verfassungsrechtlichen Anforderungen gewährleisten. Das wäre unsere Aufgabe. Es geht nicht um eine Entfesselung des Gesetzgebers oder staatlicher Macht, sondern um eine vernünftige Rationalisierung hoheitlicher Entscheidungen. Die Beteiligung der Verwaltung bleibt konstitutiv gesichert. Mir geht es auch nicht - in einem schlanken Sinne - um Beschleunigung, sondern grundsätzlicher ansetzend um die Neujustierung des Verhältnisses von Gesetz und Verwaltung. Herr Masing hat die Kernfrage dieses Bereiches nochmal aufgeworfen: Was soll denn von wem entschieden werden? Ich würde in der Tat meinen, es geht in dieser Verschränkung nicht um eine dezisionistische Freischaltung des Parlaments, vielmehr bleibt die Verwaltung im vollen Ornat in die Sachentscheidung eingebunden. Verbesserte Legitimation und verfahrensmäßige Vereinfachung ließen sich so verbinden - das wäre der Punkt.

Letzter Punkt, damit bin ich ganz am Anfang der Diskussion. Herr Groß, Herr Hellermann, auch Herr Knauff und ganz zum Schluss Herr Fehling haben nach dem verfassungsrechtlich vorgezeichneten Umfang staatlicher Verantwortung in diesem Bereich gefragt. Meine Antwort ist zunächst einmal schlicht: Das regelt nicht die Verfassung. Das ist die Aussage, auf die ich mich mit Ihnen gerne einigen würde. Und was Art. 36 Grundrechte-Charta betrifft: Der Zugang zu bestehenden Infrastrukturen sowie zu bestehenden Diensten sind insofern Rechte zweiter Ordnung, ebenso wie Art. 3 GG. Aus ihnen kann man nicht in einer Übersprungshandlung ableiten, dass die Verfassung entsprechende Infrastrukturen auch herzustellen habe. Das wäre meine Vorstellung von der Verfassung als Rahmenordnung.

Damit bin ich bei meinem Schluss. Im letzten Jahr, seit dem Anruf im Dezember, wurde mir alles, was auf mich einstürzte, irgendwie zu Infrastruktur. Das mag an dem Thema liegen, zeigt aber auch unsere Sozialisa- 
tion und wie wichtig wir alle dieses Forum nehmen. Chancen, aber auch gewisse Gefahren eines Staatsrechtslehrer-Referats liegen somit eng beieinander. Durch die Diskussion konnte ich nun wieder in Ihren Kreis, in das gemeinsame Gespräch zurückkehren. Dafür herzlichen Dank.

Dörr, Oliver: Schade, dass ich nach diesen Worten überhaupt noch etwas sagen muss, aber so gebieten es wohl die Regeln. Auch ich danke, wie es sich an dieser Stelle gehört, für Zuspruch, vor allem für diesen, der - das mögen jene unter Ihnen, die noch nicht referiert haben, vielleicht nicht so empfinden können - sehr gut tut nach der langen Unsicherheit. Ich danke aber auch für Kritik. Ich habe - wenn ich richtig zugehört habe - vier Gruppen zu bilden: die Themeneingrenzung, die verfassungsrechtlichen Standards, die Instrumente und das Völkerrecht - das Beste sozusagen kommt also zum Schluss.

Zur Themeneingrenzung. Herr Engel, natürlich kann man über diese kategoriale Eingrenzung, die sich nicht aus dem Gesetz ergibt, immer streiten. Mit Herrn Schoch hatte auch ich viele Wochen lang große Zweifel am Thema insgesamt: Ist das in dieser Abgeschlossenheit überhaupt ein Thema für einen Juristen? Infrastrukturrecht, gibt es das überhaupt? Ich habe versucht, das mir Mögliche an begrifflicher Schärfung und Eingrenzung zu leisten. Ich glaube, Ihr Kriterium der Netzverknüpfung oder der Netzexternalitäten ist zu eng. Ich habe das in einem Absatz erwähnt. Infrastruktur ist meines Erachtens mehr als nur das, was als Netz funktioniert oder als Netz verknüpft ist. Bei Schulen mag man heute von Schulnetzwerken sprechen, aber wenigstens ein Friedhof ist nicht vernetzt, dennoch ist er ortsgebundene Grundlage staatlicher Versorgung. Ich würde ihn gerne weiter in der Infrastruktur belassen. Auch was die Ökonomen hier machen - ein bisschen davon habe ich jetzt gelernt -, hat mich, was die Kategorie- und Begriffsbildung angeht, nicht immer überzeugt.

Herr Schoch, natürlich habe ich die Entsorgung nicht ausgeklammert, sie ging vielleicht nur etwas unter. Ich habe von Abwasserentsorgung und von Abfallbeseitigung gesprochen und bezog dies auf Versorgung in einem sehr weiten, funktionalen Sinne. Das schließt natürlich als Versorgungsleistung auch die Dienstleistungen aus dem Entsorgungsbereich ein. Selbstverständlich gehören die ortsgebundenen Anlagen der Entsorgungsdienste zur Infrastruktur und zu den Infrastrukturleistungen, gar keine Frage.

Herr Schoch und Herr Meyer mahnten, glaube ich, an, dass wir die Finanzierung ausgelassen haben. Da würde ich ähnlich wie Herr Wißmann sagen, dass wir irgendetwas ausklammern mussten, damit wir halbwegs durchkommen. Natürlich haben wir auch viele Sachgebiete ausgeklammert: Über Rundfunk und Sozialdienstleistungen im Einzelnen 
haben wir nicht gesprochen, weil sonst das Ganze doch sehr weit geworden wäre. Herr Faßbender, auch das Umweltrecht fiel diesen themenbezogenen Kürzungen zum Opfer. Ich bitte dafür um Nachsicht.

Zweiter Komplex: die verfassungsrechtlichen Standards deutscher wie europäischer Grundlage. Thomas Groß fragte: Wer konkretisiert die sozialstaatlichen Mindeststandards und wie macht man das? Da würde ich zunächst ganz einfach antworten: der staatliche Gesetzgeber in Bund und Ländern nach Maßgabe der Kompetenzordnung. Sicherlich nicht, wenn das hinter der Frage gestanden haben sollte, die Union, die hierfür keine Kompetenzgrundlage hat. Auch Art. 106 Abs. 2 AEUV, der in der Literatur gerne in diesem Zusammenhang genannt wird, bietet sicherlich keine Grundlage für die unionsautonome Konkretisierung, denn die Norm setzt die Betrauung mit einer bestimmten öffentlichen Versorgungsleistung oder einer Infrastrukturleistung voraus. Ich würde auch bei den verfassungsrechtlichen Standards - das klang mehrfach an - den großen Gestaltungsspielraum vor allem des Gesetzgebers oder - etwas weiter gefasst - des Staates betonen. Herr Zacher wies darauf hin, dass die Einhegung der Politik durch Normen nicht immer gelingt. Ich glaube, das passt sehr gut zu diesem Tenor.

$\mathrm{Zu}$ den Standards rügte weiter Herr Püttner auf das Schärfste den Abschied von den gleichwertigen Lebensverhältnissen. Ich stehe trotzdem dazu, obwohl der Gegenwind heftig ist, wenn er von Ihnen kommt. Ich glaube nicht, dass Art. 3 GG, den Sie anführten, in diesem Zusammenhang uns sehr viel hilft. Mir ging es nicht um einen subjektiv einklagbaren Anspruch - ich glaube, auch Ihnen nicht - , sondern um eine konzeptionelle Betrachtung. Ich glaube, das Konzept der gleichwertigen Lebensverhältnisse hat vielleicht für die Kontexte, in denen es in der Verfassung verwendet wird (also für die Gesetzgebungskompetenz des Bundes und für die Finanzierung), noch seine Berechtigung. Für den Versorgungsauftrag des Staates scheint mir dies zunehmend brüchiger zu werden. Ich denke, der Staat ist angesichts der großen Gestaltungsspielräume, die diesem Auftrag immanent sind, berechtigt, hier Konsequenzen zu ziehen. Ich habe am Mittwoch gelernt, dass die Betrachtung des Realbereiches ganz wichtig ist, um Normen zu verstehen. Wenn man die reale soziale Entwicklung ansieht, die schrumpfende Gesellschaft, dann kann der Staat daraus Konsequenzen ziehen - bis zu einem gewissen Minimum, über das wir uns grundrechtlich und sozialstaatlich verständigen können.

Zum Europarecht. Matthias Knauff fragte nach Art. 14 AEUV und der notwendigen Infrastruktur. Natürlich kann ich das nicht exakt bezeichnen und mich stattdessen dahin zurückziehen, dass der Begriff Infrastruktur in der Norm gar nicht vorkommt. Sondern es geht nur um Daseinsvorsorge, nicht mal das wörtlich, sondern natürlich nur übersetzt 
in den „Eurospeak“. Ich wollte nur deutlich machen, dass ich als verfassungsrechtliche Grundaufgabe und als Gestaltungsauftrag Infrastruktur dort mit erfasst sehe. Wenn die Norm von öffentlichen Dienstleistungen von allgemeinem wirtschaftlichem Interesse spricht, dann meint sie auch die Infrastrukturdienstleistungen, die gegen Entgelt - also marktgängig erbracht werden. Der weite Spielraum der Mitgliedstaaten ist hier sogar in der Norm selbst zu greifen; immerhin aber handelt es sich um ein verfassungsrechtliches Datum, das den deutschen verfassungsrechtlichen Auftrag abrundet und ergänzt - erst recht natürlich im Zusammenhang mit dem Protokoll. Man wird aus der Norm selber nicht einen bestimmten Infrastrukturstandard ableiten können, keine Frage.

Herr Fehling fragte, meine ich, nach Art. 36 Grundrechte-Charta als Ansatzpunkt für Teilhabe, eventuell sogar für Teilhabeansprüche gegen den Staat. Hier würde ich noch weiter gehen als Herr Wißmann und sagen, dass Art. 36 im falschen Text steht: Das ist offensichtlich kein subjektives Recht. Dort heißt es nämlich nur, die Union achtet und anerkennt - in der deutschen Fassung, sprachlich schon ein Graus - den Zugang zu öffentlichen Leistungen. Erstens ist also nur die Union überhaupt Adressat dieser Norm. Man wird auch mit Art. 51 Grundrechte-Charta nicht darüber hinwegkommen, weil in der Norm ausdrücklich der Adressat genannt ist. Zweitens gibt es im Text gerade keinen Ansatzpunkt für ein subjektives Recht in dem Sinne, wie wir es verstehen.

Herr Kugelmann, Herr Faßbender, der Enge der Zeit geschuldet bitte ich Sie um Nachsicht, dass ich Sie zusammenfasse. Sie fragten nach: Sind die DAWIs nicht doch stärker geklärt, vor allem durch die dazu ergangenen Richtlinien? Ich bezog mich allerdings nicht auf die Richtlinien in dem Zusammenhang, sondern auf das nicht liberalisierte Europarecht. Ich meine, dass hier - das war wohl auch Herrn Faßbenders Anliegen zwar der Einwirkungsanspruch des Europarechts umfassend ist in der Breite, wenn man so will, dass aber in der Tiefe sehr wenig dabei herauskommt, weil man Grundfreiheitsverkürzungen rechtfertigen kann durch das Allgemeininteresse an der Infrastruktur in staatlicher Verantwortung und weil das Beihilferecht mittlerweile von großzügigen Ausnahmen nur so gepflastert ist, dass von einer echten Steuerungswirkung im praktischen Ergebnis wenig bleibt. Ich stimme Herrn Faßbender zu, dass das ein Teil der internationalen Lösung ist, bitte aber zu beachten - Sie haben, glaube ich, auf die Leitlinien für die transeuropäische Energieinfrastruktur hingewiesen -, dass ich das für netzgebundene Infrastrukturen anerkannt habe. Ich meine aber, nicht alle Infrastruktur ist vernetzt, und dementsprechend muss nicht jede Lösung international sein.

Wenn ich noch eine Minute habe, würde ich gerne Norman Weiß und Herrn Nowrot antworten. Zum Völkerrecht bin ich fast versucht mit dem 
alten Briest zu sagen: „Das ist ein weites Feld, Effi.“ Norman, ich meine, man kann das regeln als res communis omnium, aber dann muss man es regeln. Das Völkerrecht tut es bisher nicht. Allgemeine Pflichten nach Art einer gewohnheitsrechtlichen res communis sehe ich nicht. Im Gegenteil: Die Regime „Meeresboden“, „Antarktis“ und „Hohe See“ sind alle vertraglicher Art (jedenfalls zum überwiegenden Teil), was dafür spricht, dass man einen Vertrag schließen muss, um ein solches Regime zu begründen. Und die Erfahrungen in der ITU vor einem Jahr sprechen nicht dafür, dass das recht bald geschieht.

Der Schritt zum „Weltinnenrecht“ baut die Brücke zu dem, was Herr Nowrot zu einer eventuellen völkerrechtlichen Gewährleistungsverantwortung sagte. Ich habe gegen das Konzept nichts einzuwenden, weiß ich doch, lieber Herr Nowrot, dass Sie in der Literatur für innovative Lösungen und Konzepte stehen. Ich möchte als Völkerrechtler aber warnen vor einer vorschnellen Parallele zu innerstaatlichen Konzepten, dass man also sehr schnell die Weltverfassung oder das Sozialstaatsprinzip auf völkerrechtlicher Ebene gespiegelt sieht. Ich glaube nicht, dass wir das Völkerrecht besser machen, wenn wir es fortschrittlich ankleiden, als es ist, indem wir staatliche Konzepte schlicht hochzonen und sagen: Das gibt es auch alles auf völkerrechtlicher Ebene. Da bin ich sehr skeptisch. Natürlich, wenn Herr Schoch von Grundrechtsvoraussetzungen spricht, welche durch Infrastruktur geschaffen werden, dann wird man das auch für völkerrechtliche Menschenrechtspflichten - soweit sie verbindlich für den betreffenden Staat gelten - sagen können. Insoweit hat jeder Staat eine Verantwortung, die faktischen Voraussetzungen für die Einlösung derjenigen Menschenrechte herzustellen, an die er gebunden ist. Aber weiter, glaube ich, geht das Völkerrecht an dieser Stelle noch nicht.

Ich bin am Ende, ach, nein, ich habe die Instrumente vergessen. Indra Spiecker zu den Rückholoptionen, darf ich das bitte nachher bilateral machen?

Herr Zacher sprach über Recht und Zukunft. Ich versuche jetzt ein ähnlich schönes Schlusswort zu finden wie Herr Wißmann, ahne jedoch, dass es mir gelingen wird. Ich weiß nicht, ob wir uns nicht überheben, wenn wir Zukunft allein durch Recht gestalten wollen. Vielleicht können wir wenigstens Recht so ausgestalten, dass es der Zukunft nicht im Wege steht. Oder wir könnten mit Dr. Udo Brömme sagen: Zukunft ist gut für alle! Vielen herzlichen Dank. 